\title{
DEVELOPMENT OF AN EXPERIMENTAL APPARATUS FOR STUDYING HIGH-TEMPERATURE HEAT AND MASS TRANSFER IN SOILS
}

\author{
by
}

\author{
Mohsen Hedayati-Dezfooli
}

\begin{abstract}
Master of Applied Science in Mechanical Engineering, Ryerson University (2010)
Bachelor of Engineering, Ryerson University (2008)
\end{abstract}

\author{
A dissertation presented to \\ Ryerson University \\ in partial fulfillment of the \\ requirements for the degree of \\ DOCTOR OF PHILOSOPHY
}

in the program of

Mechanical and Industrial Engineering

Toronto, Ontario, Canada, 2016

CMohsen Hedayati-Dezfooli, 2016 


\section{AUTHOR'S DECLARATION FOR ELECTRONIC SUBMISSION OF A DISSERTATION}

I HEREBY DECLARE THAT I AM THE SOLE AUTHOR OF THIS DISSERTATION. THIS IS A TRUE COPY OF THE DISSERTATION, INCLUDING ANY REQUIRED FINAL REVISIONS, AS ACCEPTED BY MY EXAMINERS.

I AUTHORIZE RYERSON UNIVERSITY TO LEND THIS DISSERTATION TO OTHER INSTITUTIONS OR INDIVIDUALS FOR THE PURPOSE OF SCHOLARLY RESEARCH.

I FURTHER AUTHORIZE RYERSON UNIVERSITY TO REPRODUCE THIS DISSERTATION BY PHOTOCOPYING OR BY OTHER MEANS, IN TOTAL OR IN PART, AT THE REQUEST OF OTHER INSTITUTIONS OR INDIVIDUALS FOR THE PURPOSE OF SCHOLARLY RESEARCH.

I UNDERSTAND THAT MY DISSERTATION MAY BE MADE ELECTRONICALLY AVAILABLE TO THE PUBLIC. 


\title{
DEVELOPMENT OF AN EXPERIMENTAL APPARATUS FOR STUDYING HIGH- TEMPERATURE HEAT AND MASS TRANSFER IN SOILS
}

\author{
Mohsen Hedayati-Dezfooli \\ Doctor of Philosophy, 2016 \\ Department of Mechanical and Industrial Engineering \\ Ryerson University, Toronto, Ontario, Canada, 2016
}

\begin{abstract}
The objective of this study was to design and build an experimental apparatus for studying heat and moisture transport phenomena in soils at temperatures greater than $40^{\circ} \mathrm{C}$ up to $90^{\circ} \mathrm{C}$. An experimental soil cell was designed and constructed for experimental studies of one-dimensional heat and moisture transfer within a vertical soil column. The interference effect between two proximate TDR probes was examined for three types of soils and it was found that parallel TDR probes can interfere with each other if the distance between them is around $1 \mathrm{~cm}$. Also, for the samples with higher water contents, the effect of interference on the electromagnetic waveform signals is more prominent, which can result in $15 \%$ uncertainty in the measurement of water content. Through the numerical study, four stages of design analysis were carried out to eventually reach a satisfactory design which was deemed to meet the research objective, i.e. less than 5\% variation of heat fluxes in the radial direction along the soil cell. The experimental assessment of the final soil cell was first performed using dry Matilda soil. The temperature profile along the soil cell deviated from the linear temperature profile by $18.6 \%$ when the temperature level and gradient was high at $82.6^{\circ} \mathrm{C}$ and $90^{\circ} \mathrm{C} / \mathrm{m}$, respectively. At this condition, the difference of heat fluxes between the top and bottom heat flux meters was recorded to be $34 \%$. This case is the worst case due to the low thermal conductivity of the dry soil. The experimental assessment of the final soil cell was also done for a wet Matilda soil at a degree of saturation of about $65 \%$. The temperature profile along the soil cell had a maximum deviation of $7.7 \%$ from the linear temperature profile even when the temperature level of the soil cell was high at $82.1^{\circ} \mathrm{C}$. At this condition, the difference of heat fluxes between the top and bottom heat flux meters was recorded to be $4.2 \%$. After the reliability of the apparatus was assessed, nine cases of the wet soil were studied. The results show that the temperature gradient is the main driving force to cause moisture migration.
\end{abstract}




\section{ACKNOWLEDGMENTS}

I would like to express my deepest appreciation to my supervisor, Professor Wey Leong, for his insight and priceless support with this dissertation. Without Professor Wey Leong's persistence, guidance, and enthusiasm, this dissertation would not have been possible.

I would also like to thank Drs. Jacob Friedman and Ziad Saghir (Department of Mechanical and Industrial Engineering, Ryerson University), Dr. Huu Doan (Department of Chemical Engineering, Ryerson Univesity) and Dr. Michel Bernier (Department of Mechanical Engineering, École Polytechnique de Montréal) for serving in my dissertation examination committee.

I would also like to sincerely thank Professor Tusheng Ren of China Agricultural University, Beijing, China and Yili Lu of Iowa State University for their priceless information about the TTDR probes, guidance and recommendations throughout this research.

I would also like to sincerely thank Maria Pierini and Francisco J. Mesicek from Campbell Scientific Co. for their guidance and recommendations in setting up the digital control system.

I would also like to sincerely thank Devin Ostrom for his time and priceless assistance in advising the designs and construction of the experimental model. He is a knowledgeable and reliable technical officer of the Department of Mechanical and Industrial Engineering.

And

I would like to specially thank my parents, Mehdi and Mina, for their supports and encouragements. Last, but not least, I would like to thank my beloved wife, Bahareh, for her love, patience and encouragement. 


\section{TABLE OF CONTENTS}

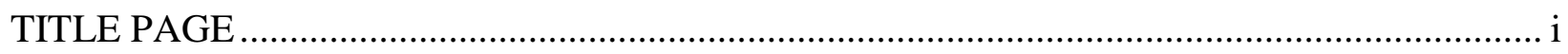

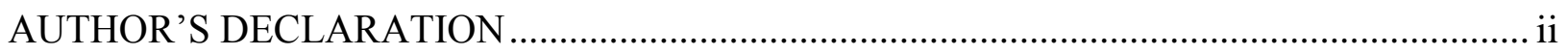

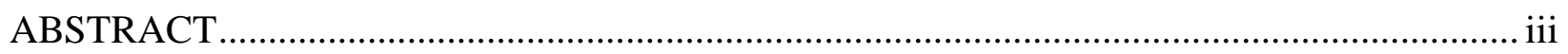

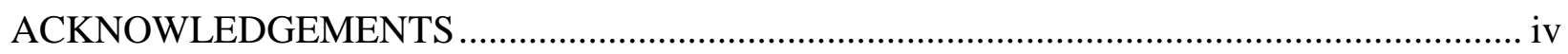

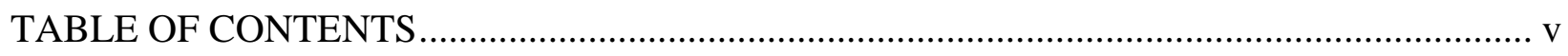

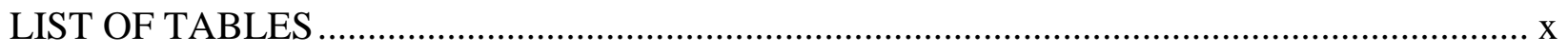

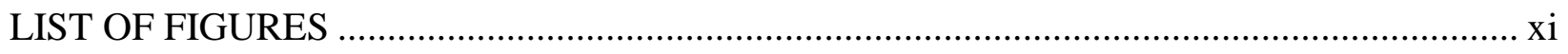

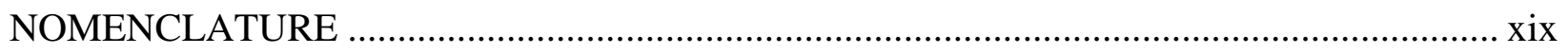

\section{CHAPTER 1 GENERAL REVIEW}

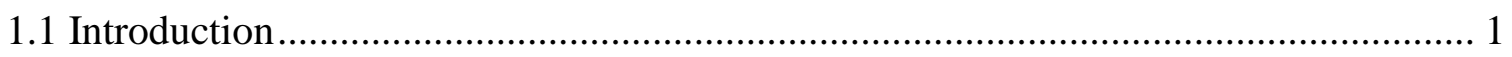

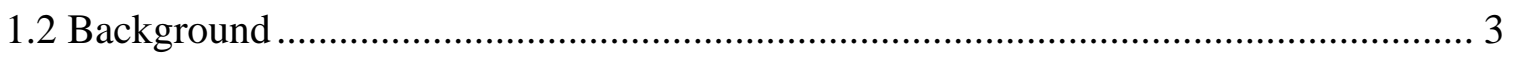

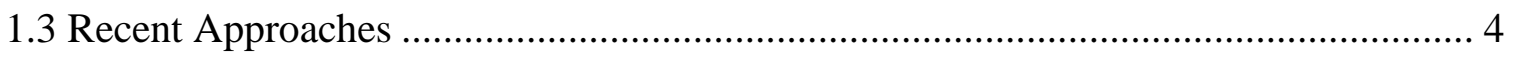

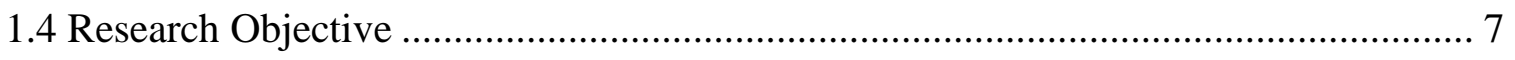

\section{CHAPTER 2 NUMERICAL STUDY ON THE DESIGN OF APPARATUS}

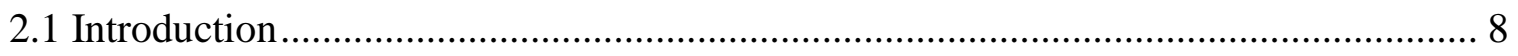

2.2 Preliminary Design of the Soil Cell Model............................................................. 8

2.3 Modified Model \#1 ……………................................................................. 10

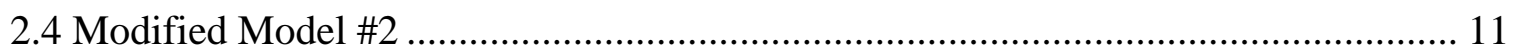

2.4.1 Material Selection for the Inner and Outer tubes ........................................ 14

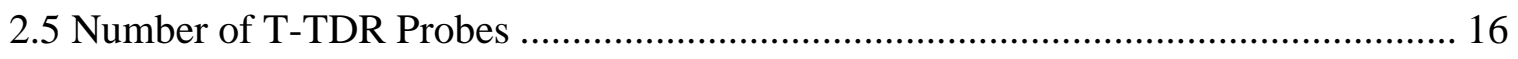


2.6 Design of the Hot and Cold plates

2.6.1 CFD Modeling of the Channel Grooved Plates ...................................... 18

2.7 Mesh Considerations of the Numerical Model ...................................................... 20

2.8 Experimental Problem of Modified Model \#2 ................................................... 20

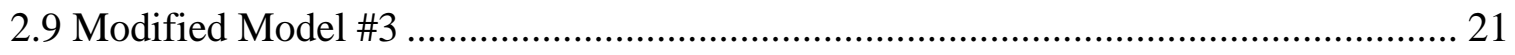

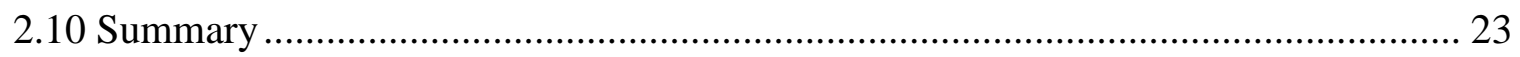

\section{CHAPTER 3 EXPERIMENTAL TECHNIQUES}

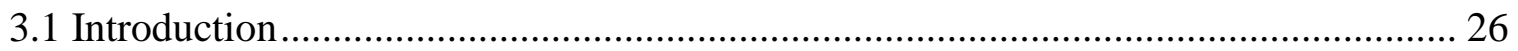

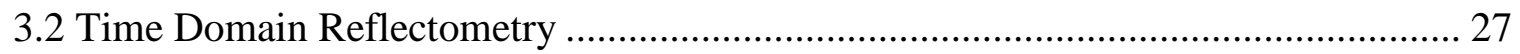

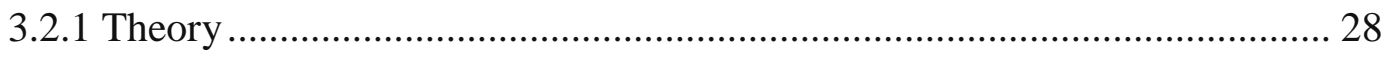

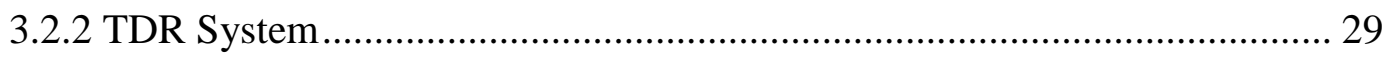

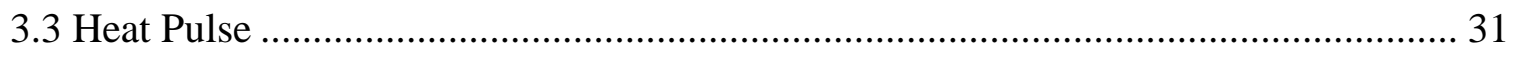

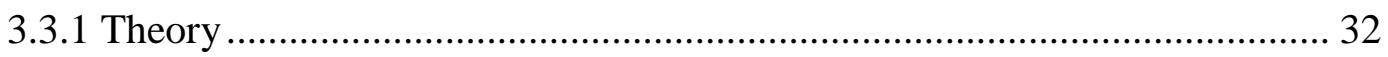

3.4 Construction Procedure of T-TDR Probe .............................................................. 33

3.4.1 Heater Needle................................................................................ 34

3.4.2 Thermocouple Needle ................................................................... 35

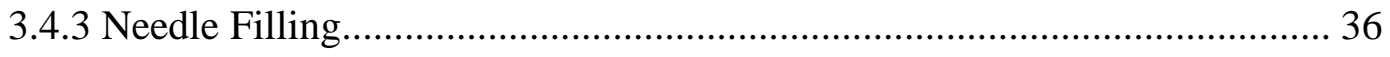

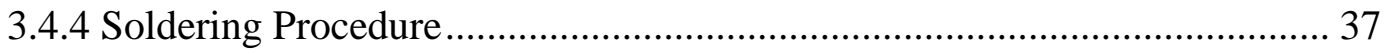

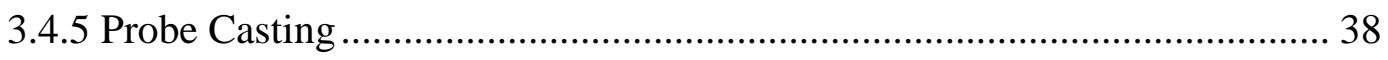

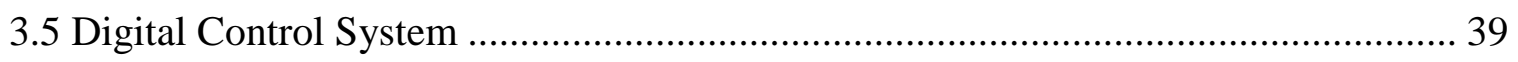

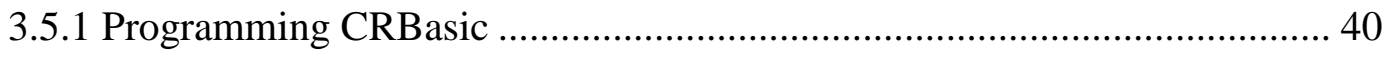

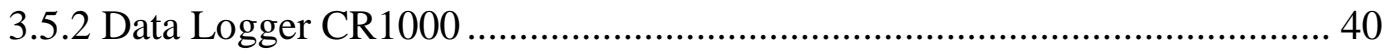




\section{CHAPTER 4 EXPERIMENTAL UNCERTAINTY ANALYSIS}

4.1 Uncertainty Analysis of Heat Pulse Technique ................................................. 42

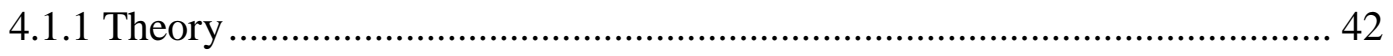

4.1.2 Measurement Uncertainty of Thermal Diffusivity ................................ 43

4.1.3 Measurement Uncertainty of Volumetric Heat Capacity.......................... 43

4.1.4 Measurement Uncertainty of Thermal Conductivity ............................... 44

4.1.5 Measurement Uncertainty of Volumetric Water Content ......................... 45

4.1.6 Summary of the Uncertainty Calculations for Soil Thermal Properties and

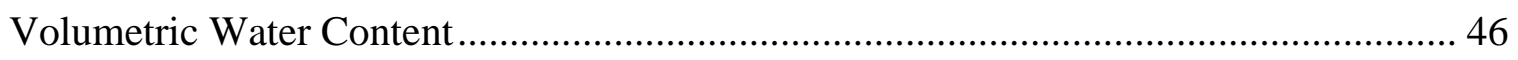

4.2 Uncertainty Analysis of Soil Sample Preparation ............................................ 47

4.2.1 Summary of the Uncertainty Calculations for Soil Sample Preparation ..... 49

4.3 Uncertainty Analysis of Temperature Measurement via T-TDR Probe .................... 50

4.3.1 Problem Definition........................................................................ 50

4.3.2 Uncertainty Caused by Heat Conduction from Thermocouple Wire........... 51

4.3.3 Uncertainty Caused by Heat Conduction from Stainless Steel Needle and

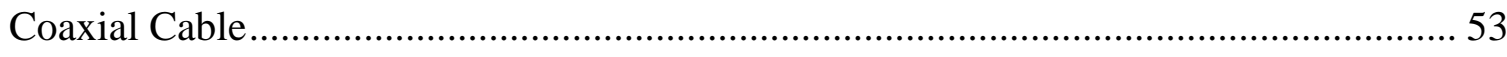

4.3.4 Calculation of the Temperature Measurement Uncertainty ....................... 55

4.3.5 Summary of the Uncertainty Calculations for Temperature Measurements 56 


\section{CHAPTER 5 EXPERIMENTAL EVALUATION OF TDR PROBES INTERFERENCE}

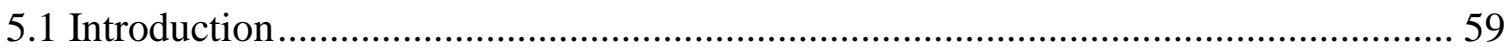

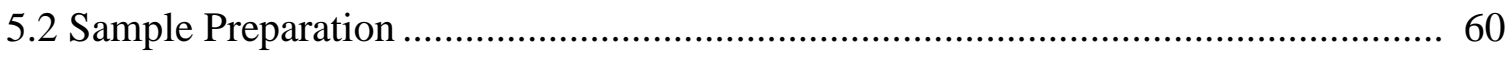

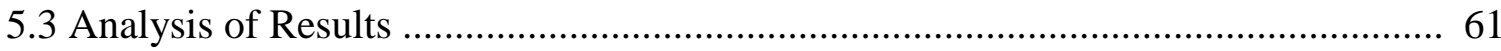

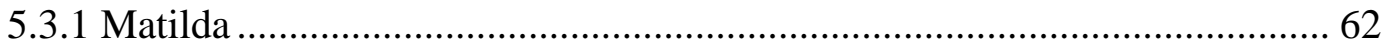

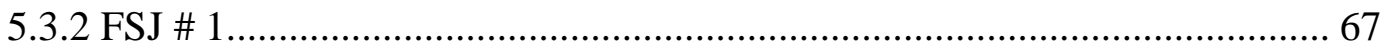

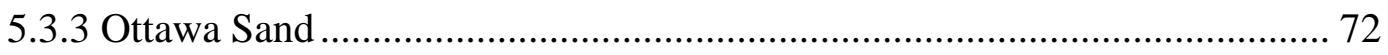

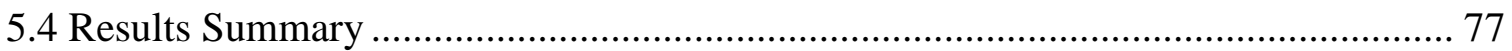

\section{CHAPTER 6 EXPERIMENTAL RESULTS OF HEAT AND MASS TRANSFER IN A} SOIL

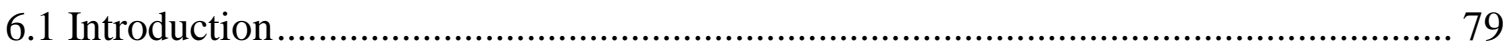

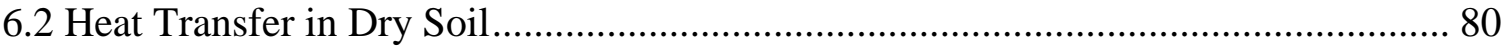

6.2.1 Observations and Analysis of Results..................................................... 80

6.3 Heat and Moisture Transfer in Wet Soil ................................................................ 84

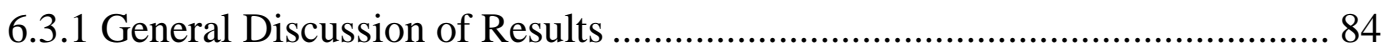

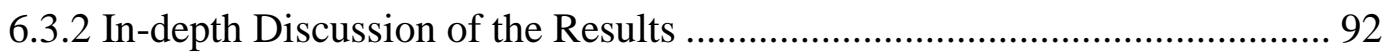

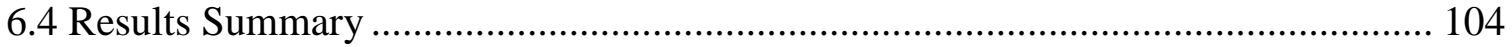

\section{CHAPTER 7 CONCLUSIONS AND FUTURE WORK}

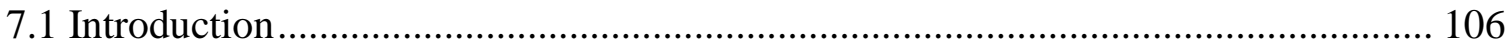

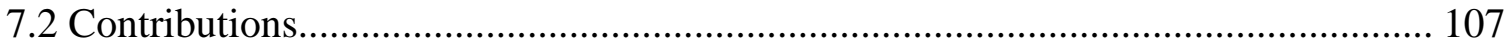

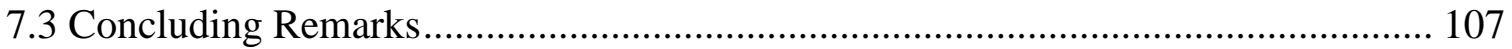


7.4 Future work

\section{APPENDIX A LIST OF MATERIALS}

A.1 List of Material for construction of T-TDR Probe 112

A.2 List of Material for apparatus construction 113

\section{APPENDIX B ENGINEERING CAD DRAWINGS}

B.1 Polyamid Plastic Mold for T-TDR Probe 115

B.2 Aluminum Alloy 2024 Plate 116

B.3 Stainless Steel Inner Cylinder for Modified Model \#2 117

B.4 Stainless Steel Outer Cylinder. 119

B.5 Stainless Steel Inner Cylinder for Modified Model \#3 120

B.6 Bolts, Nuts and Pipe connectors. 121

APPENDIX C EXPERIMENTAL PHOTOSHOOTS 122

APPENDIX D EXPERIMENTAL RESULTS OF THE WET SOIL 126

REFERENCES. 178 


\section{LIST OF TABLES}

Table 2.1 Material properties used in COMSOL simulations................................................. 14

Table 4.1 Typical input parameters used for uncertainty calculations................................... 46

Table 4.2 Results of uncertainties in measurement of the thermal properties and water content 47

Table 4.3 Overall Uncertainty in sample preparation at 95\% confidence level for ON-3 ......... 49

Table 4.4 Overall Uncertainty in sample preparation at 95\% confidence level for C-190 ......... 49

Table 4.5 Overall Uncertainty in sample preparation at $95 \%$ confidence level for BC-1 .......... 50

Table 4.6 Input data for uncertainty calculations due to conduction heat transfer ................... 57

Table 5.1: Summary of the Results of TDR Interference ............................................... 78 


\section{LIST OF FIGURES}

Fig. 2.1 Sketch of the preliminary design model ................................................................ 10

Fig. 2.2 Sketch of the modified model \#1 ….................................................................. 11

Fig. 2.3 Sketch of the modified model \#2 secured in between the hot and cold plates............... 12

Fig. 2.4 Heat flux distribution over radial direction at two different heights in the soil column at the temperature level of $82.5^{\circ} \mathrm{C}$ and temperature difference of $15^{\circ} \mathrm{C}$ 12

Fig. 2.5 Numerical model simulated in COMSOL: a) boundary conditions, b) materials, c) finiteelement mesh of the model. 13

Fig. 2.6 COMSOL results of the temperature variations along the centerline of the soil cell for the modified model \#2 with three different tube materials

Fig. 2.7 Views of heat flux vectors and isotherms of the apparatus from COMSOL simulation for the modified model \#2 with three different tube materials: a) stainless steel 304, b) aluminum alloy 2024 and c) PEEK. 16

Fig. 2.8 Depiction of channel grooved on aluminum alloy 2024 plate 19

Fig. 2.9 3D finite-element mesh structure of the water channel on aluminum plate. 19

Fig. 2.10 Sketch of cross section of the modified model \#3. 22

Fig. 2.11 Views of heat flux vectors and isotherms of the apparatus from COMSOL simulation for the modified model \#3 23

Fig. 3.1 An example of tangent line method used to find apparent probe length $\left(L_{a}\right)$ from the reflected waveform 28

Fig. 3.2 Illustration of PC-TDR toolbox to find cable and window lengths 31

Fig. 3.3 Sample graph of temperature rise variations vs. time 32

Fig. 3.4 An in-house made T-TDR Probe. 34

Fig. 3.5 The photo shoots of uncompleted T-TDR probe during the process of construction .... 35

Fig. 3.6 Flow chart of experimental setup algorithm. 40

Fig. 4.1 Schematic of heat conduction problem as modelled: The probe's needles are inserted in soil, portion of coaxial cable is surrounded by insulation and extended infinitely into the air .... 51 
Fig. 4.2 Schematic of thermal resistance network between bare copper wire and soil medium .. 53

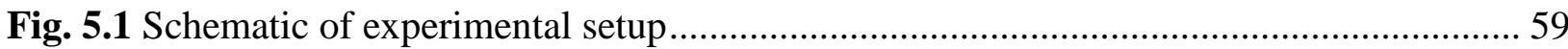

Fig. 5.2 Soil sample C-190 with inserted TDR probes at $1 \mathrm{~cm}$ and $4.2 \mathrm{~cm}$ apart .................... 61

Fig. 5.3 The waveforms captured simultaneously by the probes placing 2.2, 3.2 and $4.2 \mathrm{~cm}$ apart in ON-3 with $\mathrm{S}_{\mathrm{r}}=0.25$

Fig. 5.4 The waveforms captured simultaneously by the probes placing $1.0 \mathrm{~cm}$ apart in $\mathrm{ON}-3$ with $\mathrm{S}_{\mathrm{r}}=0.25$

Fig. 5.5 The waveforms captured simultaneously by the probes placing 2.2, 3.2 and $4.2 \mathrm{~cm}$ apart in $\mathrm{ON}-3$ with $\mathrm{S}_{\mathrm{r}}=0.5$

Fig. 5.6 The waveforms captured simultaneously by the probes placing $1.0 \mathrm{~cm}$ apart in $\mathrm{ON}-3$ with $\mathrm{S}_{\mathrm{r}}=0.5$

Fig. 5.7 The waveforms captured simultaneously by the probes placing 2.2, 3.2 and $4.2 \mathrm{~cm}$ apart in $\mathrm{ON}-3$ with $\mathrm{S}_{\mathrm{r}}=1$ 66

Fig. 5.8 The waveforms captured simultaneously by the probes placing $1.0 \mathrm{~cm}$ apart in ON-3 with $\mathrm{S}_{\mathrm{r}}=1$ 66

Fig. 5.9 The waveforms captured simultaneously by the probes placing 2.2, 3.2 and $4.2 \mathrm{~cm}$ apart in $\mathrm{BC}-1$ soil with $\mathrm{S}_{\mathrm{r}}=0.25$.....

Fig. 5.10 The waveforms captured simultaneously by the probes placing $1.0 \mathrm{~cm}$ apart in BC-1soil with $S_{\mathrm{r}}=0.25$.

Fig. 5.11 The waveforms captured simultaneously by the probes placing 2.2, 3.2 and $4.2 \mathrm{~cm}$ apart in $\mathrm{BC}-1$ soil with $\mathrm{S}_{\mathrm{r}}=0.5$.....

Fig. 5.12 The waveforms captured simultaneously by the probes placing $1.0 \mathrm{~cm}$ apart in $\mathrm{BC}-1$ soil with $S_{\mathrm{r}}=0.5$

Fig. 5.13 The waveforms captured simultaneously by the probes placing 2.2, 3.2 and $4.2 \mathrm{~cm}$ apart in BC-1 soil with $S_{\mathrm{r}}=1$

Fig. 5.14 The waveforms captured simultaneously by the probes placing $1.0 \mathrm{~cm}$ apart in BC-1 soil with $S_{\mathrm{r}}=1$

Fig. 5.15 The waveforms captured simultaneously by the probes placing 2.2, 3.2 and $4.2 \mathrm{~cm}$ apart in C-190 soil with $\mathrm{S}_{\mathrm{r}}=0.25$ 72 
Fig. 5.16 The waveforms captured simultaneously by the probes placing $1.0 \mathrm{~cm}$ apart in C-190 soil with $S_{\mathrm{r}}=0.25$

Fig. 5.17 The waveforms captured simultaneously by the probes placing 2.2, 3.2 and $4.2 \mathrm{~cm}$ apart in C-190 soil with $\mathrm{S}_{\mathrm{r}}=0.5$.

Fig. 5.18 The waveforms captured simultaneously by the probes placing $1.0 \mathrm{~cm}$ apart in C-190 soil with $\mathrm{S}_{\mathrm{r}}=0.5$.

Fig. 5.19 The waveforms captured simultaneously by the probes placing 2.2, 3.2 and $4.2 \mathrm{~cm}$ apart in C-190 soil with $S_{\mathrm{r}}=1$.

Fig. 5.20 The waveforms captured simultaneously by the probes placing $1.0 \mathrm{~cm}$ apart in C-190 soil with $\mathrm{S}_{\mathrm{r}}=1$

Fig. 6.1 Temperature variations along dry soil column from the top, where the distance is $0 \mathrm{~mm}$, to the bottom, where the distance is $147.9 \mathrm{~mm}$ at the temperature level of $37.9^{\circ} \mathrm{C}$ and the temperature difference of $13.4^{\circ} \mathrm{C}$ 81

Fig. 6.2 Temperature variations along dry soil column from the top, where the distance is $0 \mathrm{~mm}$, to the bottom, where the distance is $147.9 \mathrm{~mm}$ at the temperature level of $52.9^{\circ} \mathrm{C}$ and the temperature difference of $13.1^{\circ} \mathrm{C}$ 81

Fig. 6.3 Temperature variations along dry soil column from the top, where the distance is $0 \mathrm{~mm}$, to the bottom, where the distance is $147.9 \mathrm{~mm}$ at the temperature level of $67.8{ }^{\circ} \mathrm{C}$ and the temperature difference of $13.3^{\circ} \mathrm{C}$ 82

Fig. 6.4 Temperature variations along dry soil column from the top, where the distance is $0 \mathrm{~mm}$, to the bottom, where the distance is $147.9 \mathrm{~mm}$ at the temperature level of $82.6{ }^{\circ} \mathrm{C}$ and the temperature difference of $13.5^{\circ} \mathrm{C}$ 82

Fig. 6.5 The results of highest percent temperature deviations from the linear temperature distribution with respect to overall temperature differences and the percent difference of heat fluxes between the inlet and the outlet of dry soil cell at four different temperature levels......... 83

Fig. 6.6 The results of the highest percent temperature deviations from the linear temperature profile with respect to overall temperature differences and the percent differences of heat fluxes at four different temperature levels.

Fig. 6.7 The results of the highest percent temperature deviations from the linear temperature profile with respect to overall temperature differences and the percent differences of heat fluxes at various temperature levels and temperature differences................................................. 86

Fig. 6.8 Schematic view of soil cell's control volumes ................................................ 88

Fig. 6.9 Variations of the temperature vs. time along the soil column ................................... 88 
Fig. 6.10 Variations of the degree of saturation vs. time along the soil column 89

Fig. 6.11 Variations of the thermal properties vs. time along the soil column for the case of 90$10^{\circ} \mathrm{C}$. a) Volumetric heat capacity b) Thermal conductivity c) Thermal diffusivity .... 91

Fig. 6.12 Variations of the water content differences $(\Delta \theta)$ vs. time for the case of $90-10^{\circ} \mathrm{C}$. The symbol $\Delta \theta$ is the water content difference of control volumes between time $t$ and initial time $t_{o}$. The positive value is an indication of moisture gain and the negative value is an indication of moisture loss ...... 92

Fig. 6.13 The experimental results for control volume 1 of the soil column for the case of $90^{\circ} \mathrm{C}$ hot plate and $10^{\circ} \mathrm{C}$ cold plate 96

Fig. 6.14 The experimental results for control volume 2 of the soil column for the case of $90^{\circ} \mathrm{C}$ hot plate and $10^{\circ} \mathrm{C}$ cold plate 98

Fig. 6.15 The experimental results for control volume 3 of the soil column for the case of $90^{\circ} \mathrm{C}$ hot plate and $10^{\circ} \mathrm{C}$ cold plate 101

Fig. 6.16 The experimental results for control volume 4 of the soil column for the case of $90^{\circ} \mathrm{C}$ hot plate and $10^{\circ} \mathrm{C}$ cold plate 102

Fig. 6.17 The experimental results for control volume 5 of the soil column for the case of $90^{\circ} \mathrm{C}$ hot plate and $10^{\circ} \mathrm{C}$ cold plate 103

Fig. 6.18 Variations of the saturation degrees in percent vs. temperature gradients at control volume 1 ..... 105

Fig. C.1 Soil cell of modified model \#2 containing wet soil: (a) Soil cell without the top aluminum cap (b) Soil cell with the top aluminum cap 122

Fig. C.2 Soil cell of modified model \#3 containing wet soil: (a) Soil cell without the top stainless steel cap (b) Soil cell with the top stainless steel cap 122

Fig. C.3 From left to right: (a) Top view of the closed cap soil cell of modified model \#3, containing wet soil, with inserted in-house made T-TDR probes which is surrounded by outer stainless steel tube, (b) Top view of the opened cap soil cell of modified model \#3, containing wet soil, with inserted in-house made T-TDR probes which is surrounded by outer stainless steel tube (as shown, thin HFM is attached to the surface of the thin stainless steel plate)..... 123

Fig. C.4 From left to right: (a) Top view of modified model \#3 soil cell which is surrounded by fiber glass insulation and outer stainless steel tube, (b) Side view of the apparatus without outer insulation layer and (c) Side view of the apparatus with outer insulation layer 123

Fig. C.5 T-TDR probe mold casting clamped on the support bar 124 
Fig. C.6 Campbell Scientific digital system includes SDMS-CD16S (relay controller), Data Logger (CR-1000), TDR-100 and AM25T (multiplexer for the thermocouples)

Fig. C.7 Campbell Scientific digital system and DC power supply connected to the PC via 8 conductor cable from Data Logger $(\mathrm{CR}-1000)$................................................................ 125

Fig. C.8 Lauda Proline RP1845 Thermal Baths .............................................................. 125

Fig. D.1 The experimental results for control volume 1 of the soil column for the case of $45^{\circ} \mathrm{C}$ hot plate and $30^{\circ} \mathrm{C}$ cold plate 126

Fig. D.2 The experimental results for control volume 2 of the soil column for the case of $45^{\circ} \mathrm{C}$ hot plate and $30^{\circ} \mathrm{C}$ cold plate 127

Fig. D.3 The experimental results for control volume 3 of the soil column for the case of $45^{\circ} \mathrm{C}$ hot plate and $30^{\circ} \mathrm{C}$ cold plate. 128

Fig. D.4 The experimental results for control volume 4 of the soil column for the case of $45^{\circ} \mathrm{C}$ hot plate and $30^{\circ} \mathrm{C}$ cold plate. 129

Fig. D.5 The experimental results for control volume 5 of the soil column for the case of $45^{\circ} \mathrm{C}$ hot plate and $30^{\circ} \mathrm{C}$ cold plate. 130

Fig. D.6 The experimental results for control volume 1 of the soil column for the case of $60^{\circ} \mathrm{C}$ hot plate and $45^{\circ} \mathrm{C}$ cold plate. 131

Fig. D.7 The experimental results for control volume 2 of the soil column for the case of $60^{\circ} \mathrm{C}$ hot plate and $45^{\circ} \mathrm{C}$ cold plate. 132

Fig. D.8 The experimental results for control volume 3 of the soil column for the case of $60^{\circ} \mathrm{C}$ hot plate and $45^{\circ} \mathrm{C}$ cold plate. 133

Fig. D.9 The experimental results for control volume 4 of the soil column for the case of $60^{\circ} \mathrm{C}$ hot plate and $45^{\circ} \mathrm{C}$ cold plate. 134

Fig. D.10 The experimental results for control volume 5 of the soil column for the case of $60^{\circ} \mathrm{C}$ hot plate and $45^{\circ} \mathrm{C}$ cold plate. 135

Fig. D.11 The experimental results for control volume 1 of the soil column for the case of $75^{\circ} \mathrm{C}$ hot plate and $60^{\circ} \mathrm{C}$ cold plate. 136

Fig. D.12 The experimental results for control volume 2 of the soil column for the case of $75^{\circ} \mathrm{C}$ hot plate and $60^{\circ} \mathrm{C}$ cold plate. 137

Fig. D.13 The experimental results for control volume 3 of the soil column for the case of $75^{\circ} \mathrm{C}$ hot plate and $60^{\circ} \mathrm{C}$ cold plate. 138

Fig. D.14 The experimental results for control volume 4 of the soil column for the case of $75^{\circ} \mathrm{C}$ hot plate and $60^{\circ} \mathrm{C}$ cold plate. 139 
Fig. D.15 The experimental results for control volume 5 of the soil column for the case of $75^{\circ} \mathrm{C}$ hot plate and $60^{\circ} \mathrm{C}$ cold plate.

Fig. D.16 The experimental results for control volume 1 of the soil column for the case of $90^{\circ} \mathrm{C}$ hot plate and $75^{\circ} \mathrm{C}$ cold plate.

Fig. D.17 The experimental results for control volume 2 of the soil column for the case of $90^{\circ} \mathrm{C}$ hot plate and $75^{\circ} \mathrm{C}$ cold plate. 142

Fig. D.18 The experimental results for control volume 3 of the soil column for the case of $90^{\circ} \mathrm{C}$ hot plate and $75^{\circ} \mathrm{C}$ cold plate.

Fig. D.19 The experimental results for control volume 4 of the soil column for the case of $90^{\circ} \mathrm{C}$ hot plate and $75^{\circ} \mathrm{C}$ cold plate.

Fig. D.20 The experimental results for control volume 5 of the soil column for the case of $90^{\circ} \mathrm{C}$ hot plate and $75^{\circ} \mathrm{C}$ cold plate.

Fig. D.21 The experimental results for control volume 1 of the soil column for the case of $50^{\circ} \mathrm{C}$ hot plate and $30^{\circ} \mathrm{C}$ cold plate. 146

Fig. D.22 The experimental results for control volume 2 of the soil column for the case of $50^{\circ} \mathrm{C}$ hot plate and $30^{\circ} \mathrm{C}$ cold plate.

Fig. D.23 The experimental results for control volume 3 of the soil column for the case of $50^{\circ} \mathrm{C}$ hot plate and $30^{\circ} \mathrm{C}$ cold plate. 148

Fig. D.24 The experimental results for control volume 4 of the soil column for the case of $50^{\circ} \mathrm{C}$ hot plate and $30^{\circ} \mathrm{C}$ cold plate.

Fig. D.25 The experimental results for control volume 5 of the soil column for the case of $50^{\circ} \mathrm{C}$ hot plate and $30^{\circ} \mathrm{C}$ cold plate.

Fig. D.26 The experimental results for control volume 1 of the soil column for the case of $60^{\circ} \mathrm{C}$ hot plate and $20^{\circ} \mathrm{C}$ cold plate.

Fig. D.27 The experimental results for control volume 2 of the soil column for the case of $60^{\circ} \mathrm{C}$ hot plate and $20^{\circ} \mathrm{C}$ cold plate.

Fig. D.28 The experimental results for control volume 3 of the soil column for the case of $60^{\circ} \mathrm{C}$ hot plate and $20^{\circ} \mathrm{C}$ cold plate.

Fig. D.29 The experimental results for control volume 4 of the soil column for the case of $60^{\circ} \mathrm{C}$ hot plate and $20^{\circ} \mathrm{C}$ cold plate.

Fig. D.30 The experimental results for control volume 5 of the soil column for the case of $60^{\circ} \mathrm{C}$ hot plate and $20^{\circ} \mathrm{C}$ cold plate. 155

Fig. D.31 The experimental results for control volume 1 of the soil column for the case of $70^{\circ} \mathrm{C}$ hot plate and $10^{\circ} \mathrm{C}$ cold plate. 156 
Fig. D.32 The experimental results for control volume 2 of the soil column for the case of $70^{\circ} \mathrm{C}$ hot plate and $10^{\circ} \mathrm{C}$ cold plate.

Fig. D.33 The experimental results for control volume 3 of the soil column for the case of $70^{\circ} \mathrm{C}$ hot plate and $10^{\circ} \mathrm{C}$ cold plate.

Fig. D.34 The experimental results for control volume 4 of the soil column for the case of $70^{\circ} \mathrm{C}$ hot plate and $10^{\circ} \mathrm{C}$ cold plate.

Fig. D.35 The experimental results for control volume 5 of the soil column for the case of $70^{\circ} \mathrm{C}$ hot plate and $10^{\circ} \mathrm{C}$ cold plate.

Fig. D.36 The experimental results for control volume 1 of the soil column for the case of $80^{\circ} \mathrm{C}$ hot plate and $10^{\circ} \mathrm{C}$ cold plate. 161

Fig. D.37 The experimental results for control volume 2 of the soil column for the case of $80^{\circ} \mathrm{C}$ hot plate and $10^{\circ} \mathrm{C}$ cold plate. 162

Fig. D.38 The experimental results for control volume 3 of the soil column for the case of $80^{\circ} \mathrm{C}$ hot plate and $10^{\circ} \mathrm{C}$ cold plate. 163

Fig. D.39 The experimental results for control volume 4 of the soil column for the case of $80^{\circ} \mathrm{C}$ hot plate and $10^{\circ} \mathrm{C}$ cold plate. 164

Fig. D.40 The experimental results for control volume 5 of the soil column for the case of $80^{\circ} \mathrm{C}$ hot plate and $10^{\circ} \mathrm{C}$ cold plate. 165

Fig. D.41 Variations of the water content differences $(\Delta \theta)$ vs. time for the case of $45-30^{\circ} \mathrm{C}$. The symbol $\Delta \theta$ is the water content difference of control volumes between time $t$ and initial time $t_{o}$. The positive value is an indication of moisture gain and the negative value is an indication of moisture loss 166

Fig. D.42 Variations of the water content differences $(\Delta \theta)$ vs. time for the case of $60-45^{\circ} \mathrm{C}$. The symbol $\Delta \theta$ is the water content difference of control volumes between time $t$ and initial time $t_{o}$. The positive value is an indication of moisture gain and the negative value is an indication of moisture loss 166

Fig. D.43 Variations of the water content differences $(\Delta \theta)$ vs. time for the case of $75-60^{\circ} \mathrm{C}$. The symbol $\Delta \theta$ is the water content difference of control volumes between time $t$ and initial time $t_{o}$. The positive value is an indication of moisture gain and the negative value is an indication of moisture loss 167

Fig. D.44 Variations of the water content differences $(\Delta \theta)$ vs. time for the case of $90-75^{\circ} \mathrm{C}$. The symbol $\Delta \theta$ is the water content difference of control volumes between time $t$ and initial time $t_{o}$. The positive value is an indication of moisture gain and the negative value is an indication of moisture loss 167

Fig. D.45 Variations of the water content differences $(\Delta \theta)$ vs. time for the case of $50-30^{\circ} \mathrm{C}$. The symbol $\Delta \theta$ is the water content difference of control volumes between time $t$ and initial time $t_{o}$. 
The positive value is an indication of moisture gain and the negative value is an indication of moisture loss 168

Fig. D.46 Variations of the water content differences $(\Delta \theta)$ vs. time for the case of $60-20^{\circ} \mathrm{C}$. The symbol $\Delta \theta$ is the water content difference of control volumes between time $t$ and initial time $t_{o}$. The positive value is an indication of moisture gain and the negative value is an indication of moisture loss 168

Fig. D.47 Variations of the water content differences $(\Delta \theta)$ vs. time for the case of $70-10^{\circ} \mathrm{C}$. The symbol $\Delta \theta$ is the water content difference of control volumes between time $t$ and initial time $t_{o}$. The positive value is an indication of moisture gain and the negative value is an indication of moisture loss 169

Fig. D.48 Variations of the water content differences $(\Delta \theta)$ vs. time for the case of $80-10^{\circ} \mathrm{C}$. The symbol $\Delta \theta$ is the water content difference of control volumes between time $t$ and initial time $t_{o}$. The positive value is an indication of moisture gain and the negative value is an indication of moisture loss 169

Fig. D.49 Variations of the thermal properties vs. time along the soil column for the case of 45$30^{\circ} \mathrm{C}$. a) Volumetric heat capacity b) Thermal conductivity c) Thermal diffusivity 170

Fig. D.50 Variations of the thermal properties vs. time along the soil column for the case of 60$45^{\circ} \mathrm{C}$. a) Volumetric heat capacity b) Thermal conductivity c) Thermal diffusivity 171

Fig. D.51 Variations of the thermal properties vs. time along the soil column for the case of 75$60^{\circ} \mathrm{C}$. a) Volumetric heat capacity b) Thermal conductivity c) Thermal diffusivity 172

Fig. D.52 Variations of the thermal properties vs. time along the soil column for the case of 90$75^{\circ} \mathrm{C}$. a) Volumetric heat capacity b) Thermal conductivity c) Thermal diffusivity 173

Fig. D.53 Variations of the thermal properties vs. time along the soil column for the case of 50$30^{\circ} \mathrm{C}$. a) Volumetric heat capacity b) Thermal conductivity c) Thermal diffusivity 174

Fig. D.54 Variations of the thermal properties vs. time along the soil column for the case of 60$20^{\circ} \mathrm{C}$. a) Volumetric heat capacity b) Thermal conductivity c) Thermal diffusivity 175

Fig. D.55 Variations of the thermal properties vs. time along the soil column for the case of 70$10^{\circ} \mathrm{C}$. a) Volumetric heat capacity b) Thermal conductivity c) Thermal diffusivity 176

Fig. D.56 Variations of the thermal properties vs. time along the soil column for the case of 80$10^{\circ} \mathrm{C}$. a) Volumetric heat capacity b) Thermal conductivity c) Thermal diffusivity 


\section{NOMENCLATURE}

\begin{tabular}{|c|c|}
\hline C & Volumetric heat capacity $\left(\mathrm{J} / \mathrm{m}^{3} \cdot \mathrm{K}\right)$ \\
\hline$Q$ & Total heat transfer $(\mathrm{J} / \mathrm{s})$ \\
\hline $\mathrm{V}$ & Volume $\left(\mathrm{m}^{3}\right)$ \\
\hline$c_{w}$ & Specific heat capacity of water $(\mathrm{J} / \mathrm{kg} \cdot \mathrm{K})$ \\
\hline$c_{s}$ & Specific heat of soil solids $(\mathrm{J} / \mathrm{kg} \cdot \mathrm{K})$ \\
\hline$d$ & Distance from the emitter (m) \\
\hline$D_{a}$ & Soil thermal diffusivity $\left(\mathrm{m}^{2} / \mathrm{s}\right)$ \\
\hline$D_{\theta l}$ & Moisture diffusivity due to moisture gradient $\left(\mathrm{m}^{2} / \mathrm{s}\right)$ \\
\hline$D_{T l}$ & Moisture diffusivity due to temperature gradient $\left(\mathrm{m}^{2} / \mathrm{s} \cdot \mathrm{K}\right)$ \\
\hline$K_{h}$ & Hydraulic conductivity $(\mathrm{m} / \mathrm{s})$ \\
\hline$\dot{m}$ & Mass flow rate (kg/s) \\
\hline$g$ & Gravitational acceleration $\left(\mathrm{m} / \mathrm{s}^{2}\right)$ \\
\hline$k$ & Thermal conductivity $(\mathrm{W} / \mathrm{m} \cdot \mathrm{K})$ \\
\hline$t$ & Time (s) \\
\hline$t_{\mathrm{o}}$ & Duration of heat pulse (s) \\
\hline$t_{m}$ & Time in which temperature reaches to its maximum (s) \\
\hline$T$ & Temperature $\left(\mathrm{K}\right.$ or $\left.{ }^{\circ} \mathrm{C}\right)$ \\
\hline$x$ & Vertical distance (m) \\
\hline$\eta$ & Soil porosity ( $\mathrm{m}^{3}$ of pore space $/ \mathrm{m}^{3}$ of soil) \\
\hline$\rho$ & Density $\left(\mathrm{kg} / \mathrm{m}^{3}\right)$ \\
\hline
\end{tabular}




\begin{tabular}{|c|c|}
\hline$\rho_{b}$ & Soil bulk density $\left(\mathrm{kg} / \mathrm{m}^{3}\right)$ \\
\hline$\rho_{s}$ & Density of soil solid $\left(\mathrm{kg} / \mathrm{m}^{3}\right)$ \\
\hline$\rho_{w}$ & Density of water $\left(\mathrm{kg} / \mathrm{m}^{3}\right)$ \\
\hline$S_{r}$ & Degree of saturation $\left(\mathrm{m}^{3}\right.$ of water $/ \mathrm{m}^{3}$ of pore space) \\
\hline$\theta$ & Volumetric water content $\left(\mathrm{m}^{3} / \mathrm{m}^{3}\right)$ \\
\hline$L_{a}$ & Apparent length of electromagnetic wave (m) \\
\hline$L$ & Length of emitter (m) \\
\hline$K$ & Dielectric constant (dimensionless) \\
\hline$v$ & Velocity $(\mathrm{m} / \mathrm{s})$ \\
\hline$q_{l}$ & Liquid flux $\left(\mathrm{kg} / \mathrm{m}^{2} \cdot \mathrm{s}\right)$ \\
\hline$q$ & Energy per unit length of the emitter $(\mathrm{J} / \mathrm{m} \cdot \mathrm{s})$ \\
\hline$\Delta T_{m}$ & Maximum temperature difference $(\mathrm{K})$ \\
\hline$h$ & Conductive heat transfer coefficient $\left(\mathrm{W} / \mathrm{m}^{2} \cdot \mathrm{K}\right)$ \\
\hline$R$ & Electrical resistance $(\Omega)$ \\
\hline
\end{tabular}




\section{CHAPTER 1}

\section{GENERAL REVIEW}

\subsection{Introduction}

Ground thermal energy storage (GTES) has become an interesting research topic and particular interest now is in "high-temperature ground thermal energy storage". In order to fully utilize the solar energy or industrial waste heat, it is beneficial to store it in high-temperature ground; so, for instance, for the purpose of space heating, the stored heat can be retrieved directly without the need of using a ground source heat pump. This would highly improve the efficiency of such systems. The Drake Landing Solar Community is a community in Okotoks, Alberta, Canada, equipped with a central solar heating system and other energy efficient technology. It is a first-in-the-world example of successful application of seasonal high-temperature GTES system with over $90 \%$ of residential space heating needs being met by solar thermal energy which is collected and stored in the ground at high temperatures up to $80^{\circ} \mathrm{C}$ over the summer season. In this project, the GTES contains mostly clay which has low moisture diffusivity so that it is able to retain its moisture content and ability to store the heat. However, not everywhere would have such type of soil. Therefore, a fundamental study in high-temperature heat and moisture transfer in various types of soils is necessary to aid the design of such systems.

Soil is considered, in a strict sense, a non-homogeneous and non-isotropic porous material. The term soil, as used by engineers, refers to a complicated material consisting of solid particles of various compositions (mineral and organic) and various shapes and sizes that are randomly arranged with pore spaces between them. These pores contain air and usually water in its various phases as vapor, liquid or ice. The composition of naturally occurring soil varies continuously because of changes in the amount and phase of water at various locations. These changes result mainly from the continuously varying temperature field to which the soil is subject. The daily temperature fluctuations are superimposed on the seasonal cycle, and there is a geothermal heat flux resulting from the flow of heat upwards from the hot interior of the ground. These changing temperature gradients alter the soil composition, particularly with regard to changes in the amount,

phase and condition of water. This leads to variations in the thermal properties of the soil (Omar 1981). The study of moisture and heat distribution in soil is useful in various applications such as: 
agriculture, environmental building control, discovery and detection of buried objects and so on. Thermal gradients induce moisture transfer and so this transport will affect heat flow. Indeed moisture and temperature fields are more or less coupled. The thermal gradients produced by these temperature fields cause soil moisture to be transferred from warmer to cooler areas in both the vapor and the liquid phases. The thermally induced moisture flow may significantly affect the net transfer of the soil water and nutrients by changing the moisture content gradients and the capillary conductivity ${ }^{1}$, in addition to the direct effects of mass transfer. Thermal moisture transport may be thought of as the moisture flux through soil which arises solely due to a temperature gradient. Thermal gradients and the associated moisture transfer cause changes in moisture contents and pressures. These effects need to be taken into consideration in analysis of net moisture flow through the soil. Moisture flows in the form of liquid and vapor where the flow of the vapor phase is mainly considered as a molecular diffusion process. In unsaturated soils, thermally induced flow increases rapidly as the moisture content decreases. Indeed, the decrease in moisture content is accompanied by a decrease in the thermal liquid moisture flow and an increase in the thermal vapor moisture flow (Cary 1966). The process of heat and moisture transfer in soil is basically driven by the thermal gradients. This process forms the temperature and moisture content distribution in the soil as a porous medium. The conveyance of the latent heat ${ }^{2}$ by vapor migration through the soil and within the boundary of the soil/atmosphere is a main process which controls the coupling between the heat and the moisture transfer. Precise modeling of coupled heat and moisture transfer in a high-temperature ground thermal storage is yet wanting and so requires further studies.

\footnotetext{
${ }^{1}$ Coefficient which expresses the extent to which an unsaturated permeable medium allows flow of liquid water though its interstices under unit gradient of capillary potential.

${ }^{2}$ The energy released or absorbed by a body during constant temperature process. For example a phase change during boiling heat transfer which is defined as the input of energy required by change of state from liquid to vapor at constant temperature which is called latent heat of vaporization.
} 


\subsection{Background}

To predict heat transfer in soil under the conditions of steady and unsteady heat flow requires knowledge of the basic thermal properties of soil (Noborio 1996). The soil thermal conductivity is the rate at which heat energy flows across a unit area of the soil due to a unit temperature gradient. While the flow of heat by conduction is the predominating mechanism, all possible mechanisms are involved for the flow of heat from warmer to cooler regions. The soil composition, temperature, moisture content and structure affect the heat transfer. Generally convection and radiation have negligible effects (Martynov 1959). The heat transfer process may be affected by water phase changes in the soil. In unsaturated soils the process of evaporation along with the vapor diffusion results in condensation and subsequently heat transfer. Freezing of water or melting of ice within soils may also result in considerable latent heat effects. In many situations the transfer of moisture and heat occurs simultaneously (de Vries 1974). Heat conduction occurs in all the soil constituents. Conduction operates in air or water vapor by a process of collision between the molecules and a consequent increase in their mean kinetic energy as heat passes from warmer to cooler regions. In soil the amount of heat transferred by conduction increases as the soil dry bulk density ${ }^{3}$ increases and as its degree of saturation increases. Heat being conducted through soil will take all available paths. Paths through contacting solid generally provide the major part of conductive heat transfer but thermal contact resistance may exist. There is a thermal contact resistance that gives a sudden discontinuity in the soil temperature at the contacts between solid particles with an interstitial fluid such as a gas or liquid in the gap between contacts (de Vries 1952). Considering two parallel flat plates in contact with air as the interstitial fluid in the gaps among contacts at the plate contact interface, due to the relatively lower thermal conductivity of air than the solid plates, such an imperfect contact interface effectively resists conductive heat transfer and increases the temperature gradient across it, with a consequent reduction in the heat flow across it. Similar effects may be expected to occur in the pore spaces of the soil. Free convection of a fluid is a mass and heat transport phenomenon resulting from temperature gradients in a gravity field. Basically free convective heat transfer occurs in a fluid as its density changes due to temperature change. In contrast to free convection, forced convection heat transfer

\footnotetext{
${ }^{3}$ The ratio of the mass of the solid phase of the soil (i.e., dried soil) to its total volume (solid and pore volumes together)
} 
occurs when air or water is forced to move through the pores of soils or rocks by pressure differences. One example of a forced convection effect in the ground is groundwater flow. Groundwater flow increases heat transfer by dispersion effects. Such convection effects are usually minor in soils, but in very coarse sands they may enhance heat transfer by 20 percent in comparison with the clay type (Johansen 1975). The physics concerning the process of heat and mass transfer in soils has been a subject of importance for researchers in the past decades. The study of moisture and temperature profiles within unsaturated soil is associated with various boundary conditions. The mathematical analysis of the response of soil to atmospheric conditions is problematical since the temperature and moisture variations in the unsaturated soil rely on the parameters in the transport equations, which in turn depend on the temperature and moisture content (Keyhani and Wulfsohn 2001). The pioneers in modeling coupled heat and mass transfer in porous media are Philip and de-Vries (1957) and Luikov (1964). Philip and de Vries (1957) came up with theoretical expressions for the thermal moisture and isothermal moisture diffusivities which occur in their governing partial differential equations of combined heat and moisture transfer in which they are dependent on soil hydraulic conductivity, temperature gradients, moisture potential and soil volumetric water content. They also presented an equation of heat conduction that incorporated latent heat transfer by water vapor diffusion. Later de Vries (1958) generalized these equations by considering moisture and latent heat storage in the vapor phase and sensible heat transfer by liquid migration in the soil. Hence recent mathematical models mainly engage in modifications of Philip, de Vries and Luikov's approaches (Thomas and King 1992) which have been studied by Moukalled et al. (2006).

\subsection{Recent Approaches}

In the beginning of 1980's, Topp et al. (1980) studies showed that by use of the time domain reflectometry (TDR) technique soil water content can be measured. They found the TDR method to be a reliable technique to achieve soil moisture measurements. The water content of soil is the main factor which controls the soil dielectric constant. The dielectric constant of soil is related to the velocity of electromagnetic pulse waves which are being transmitted into the soil. So basically moisture content of soil can be determined since the velocity of a transmitted pulse is measured by TDR (Topp et al. 1980). Ren et al. (1999) developed a thermo-time domain reflectometry (T-TDR) probe to concurrently measure soil volumetric water content, thermal 
conductivity, volumetric heat capacity and thermal diffusivity. The T-TDR technique is a valuable tool for monitoring coupled heat, moisture and the heat-pulse transportation. According to their studies, since simultaneous determination of soil volumetric water content and thermal properties is necessary to study the coupled flow of water and heat in soil, time domain reflectometry has become a reliable method for measuring soil volumetric water content and bulk soil electrical conductivity. They designed and tested a modified probe for measuring volumetric water content and bulk electrical conductivity with the TDR technique. Their experimental results indicated that the probe effectively does the measurements; however, an independent calibration for the volumetric water content and dimensionless bulk dielectric constant of the soil is essential. Thermal property measurements on restrained water showed that the probe provides high accuracy results, i.e. for the typical probe design the uncertainty of estimated thermal properties was specified to be about $2 \%$. They found that the probe would measure continuous data of soil thermal properties as a function of water content even though its precision requires additional accuracy analysis. Later in 2005, Horton et al. (2005) conducted experimental measurements in soil columns of different bulk densities and water contents. Their study was conducted at the room regulated temperature of $20.3^{\circ} \mathrm{C}$ on various soil samples. The clay content of the soil samples ranged from 11.6 to $36.7 \%$. They packed their soil samples into the stainless steel rings with 50 -mm diameter and 52-mm height. They studied the heat pulse and the TDR methods for soil water content determination using T-TDR probe. By applying heat pulse method, specific heats of soil solids were determined using the specified probes and volumetric soil water content, which was assessed from the relationship of water content and heat capacity. Their experimental studies on eight different soils showed that both methods provide reliable results in terms of water content data. In their study the root mean square uncertainties in measuring volumetric water contents using TDR and heat pulse techniques were found to be 0.023 and $0.022 \mathrm{~m}^{3} / \mathrm{m}^{3}$, respectively. They found the heat pulse method more suitable in determining volumetric water content of the soils having relatively lower clay content and higher organic matters. They found that accurate determination of soil water content, using TDR technique, depends upon apparent length of the probe rod since uncertainties of the probe length are being referred to relative dielectric permittivity of the soil samples and hence soil water content. In 2007, Heitman et al. (2007) developed an instrumented closed soil cell that provides one-dimensional conditions and allows in-situ measurement of temperature and thermal conductivity under momentary boundary conditions. In their 
experimental study they observed the transient temperature, water contents and thermal conductivity distributions on the same soil sample. Their soil cell was consisted of a small tube, with inner diameters of $89-\mathrm{mm}$, surrounded by a large tube with inner diameter of 209-mm. Both tubes were having the same height of $100-\mathrm{mm}$ and were made from schedule 40 polyvinyl chloride. They examined the soil cell for several experiments using four different soil types and various momentary boundary conditions. All their experiments were conducted within the temperature range of 15 to $40^{\circ} \mathrm{C}$. In their study the root mean square uncertainties in measuring volumetric water contents using TDR and heat pulse techniques were found to be 0.020 and $0.017 \mathrm{~m}^{3} / \mathrm{m}^{3}$, respectively. Their experiments were implemented for the insulated soil cells while the cells were equipped with the T-TDR probes. Using this technique they found that temperature distributions show nonlinearity, which is consistent with non-uniform thermal properties provided by thermal moisture distribution. With respect to the permittivity of the soil in the T-TDR measurement technique, they found that the volumetric heat capacity and its change agree with post experimental samples. They also found that the temperature and thermal conductivity measurements are in accordance with the observed temperature distribution profile. In their experiments, two soil materials of different texture were used, namely sand and silt loam. The soil samples were air dried and homogenized. They used two dissimilar initial moisture contents for each soil so that four different soil combinations were obtained. The T-TDR sensors were used and installed on the sides of the soil cell perpendicular to the axis of the cells. In conclusion, their study showed that, by controlling the temperature and moisture contents, temperature distribution can be compared in one-dimensional condition. In fact the improved implementation of T-TDR technique enabled them to observe both temperature distribution and thermal conductivity variations within momentary and near-steady state conditions. In 1994 Bristow et al. (1994) established a model for radial conduction of a short-time heat pulse at a distance away from heat source. They showed that all the soil thermal properties can be obtained by measuring the temperature response at a certain distance away from the line heat source and applying their theory of short-time heat pulse. Their measurements were conducted on three kinds of dry soils and their results yielded some overestimations. Later in 1995 Bristow et al. (1995) conducted a first-order uncertainty analysis to predict how thermal properties obtained by the heat pulse method can be affected by experimental uncertainty. Their uncertainty analysis model showed that, for a typical probe's geometry and heat pulse duration, the estimated thermal properties of the soil can be as small as $2 \%$. 


\subsection{Research Objective}

Through the review of the recent approaches, no study on simultaneous heat and moisture transfer in soils was found to be conducted at high temperature levels above $40^{\circ} \mathrm{C}$. Based on the literature survey, it was seen that the available theoretical models of heat and moisture transfer has been generally developed but has never been verified for high temperature studies; therefore, the objective of this study is to develop a reliable experimental apparatus for studying heat and moisture transfer not only at low temperatures but particularly at high temperatures, i.e. up to $90^{\circ} \mathrm{C}$. In this study, the two common techniques of time domain reflectometry (TDR) and heat pulse are used to measure moisture content and thermal properties of soil. The main structure of this thesis consists of experimental apparatus design process based on numerical modeling of the apparatus according to the design criteria, construction of apparatus, experimental uncertainty analysis and experimental tests. 


\section{CHAPTER 2}

\section{NUMERICAL STUDY ON THE DESIGN OF APPARATUS}

\subsection{Introduction}

In order to reduce the uncertainty of the investigation, it is desirable to first focus on the study of one-dimensional heat and moisture transport phenomena in soils. Once the onedimensional phenomena have been studied thoroughly, one may consider a more complex study based on two- or three-dimensional experiment. Therefore, the design of an experimental apparatus in the present study was established based on the criterion of having one-dimensional heat flow within a soil cell filled with a dry soil. A dry soil possesses the greatest challenge for the design because it has the lowest thermal conductivity, and hence, it has the lowest heat flux flowing though the soil cell. In this situation, a little bit of heat loss from the soil cell can cause the onedimensional conditions to be invalid. For a cylindrical soil cell with an axial axis along its centre, the existence of one-dimensional heat flow within a soil cell can be indicated by two conditions. If both ends of the cylindrical soil cell are differentially heated, the first indication-in an ideal steady-state condition-was to have the same inflow and outflow of heat transfer rates through the soil cell, and the second indication-in an ideal steady-state condition-was to have uniform heat flux distribution at any cross section of the cell. In the present study, these conditions were considered to be met when the variations are within $\pm 5 \%$. In order to provide differential heating, the soil cell would be sandwiched between two differentially heated plates made from aluminum alloy 2024. The design principle of the heated plates was to have uniform temperature distribution over the entire surface areas of the plates. In the process of designing the experimental apparatus, all aspects of the required components as well as limited budget were also taken into consideration. In this study, with respect to the design principle, the general integrity and reliability of the models were numerically assessed using COMSOL, based on realistic geometries and boundary conditions.

\subsection{Preliminary Design of the Soil Cell Model}

Fig. 2.1 shows a sketch of the preliminary design of an experimental soil cell model. The model was simulated numerically using COMSOL with predefined boundary conditions as a 2D 
axisymmetric problem. The insulation layer (fiberglass) was assumed to have the thickness of 25.4 $\mathrm{mm}$. In the preliminary design, one soil cell with two sources of heat was considered. The soil cell is made of stainless steel 304 tube which has an inner diameter of $63.5 \mathrm{~mm}$ and thickness of 6.35 $\mathrm{mm}$. On the hot side, the first source of heat was an electrically-heated plate. The electricallyheated plate includes one resistance film heater embedded in between two thin aluminum plates each $1.5 \mathrm{~mm}$ thick. The second source of heat was the hot plate which was supposed to be heated by a hot water circulation. An insulation jacket of fiberglass was considered around the soil cell, the hot plate and the cold plate. The idea was to have the electrically-heated plates to be in direct contact with the soil. One heat flux meter (HFM) with high thermal resistance having a thickness of $5 \mathrm{~mm}$ and thermal conductivity of $0.14 \mathrm{~W} / \mathrm{m} \cdot \mathrm{K}$ was sandwiched between the electrically heated plate and the hot plate, as depicted in Fig. 2.1. The high thermal resistance HFM would ensure low heat transfer between the electrically-heated plate and the hot plate. An energy balance on the electrically-heated plate was used to obtain the amount of heat transfer to the soil. In order to have heat flowing downward through the soil column, the temperature difference between the hot plate and the electrically-heated plate must be close to zero, i.e. the HFM reading close to zero. This way the majority of heat generated by the heater is directed downward. The purpose of this setup was to precisely determine the amount of heat flowing from the electrically-heated plate to the soil, as well as to protect the HFM from direct contact with the soil so that reducing the chances of being damaged by water and water vapor during experiments.

To examine the design, the model was built in COMSOL and the temperatures of the electrically-heated plates and the hot and cold plates were set at the desired values of $90^{\circ} \mathrm{C}$ and $75^{\circ} \mathrm{C}$, respectively. After running several preliminary simulations, the results showed that the differences of energy balance, i.e. percent difference between average heat fluxes entering on top of the soil column and exiting from the bottom, remain as high as $218 \%$. Since the dry soil has relatively low thermal conductivity compared to aluminum and stainless steel, most of the heat would bypass from the soil to the stainless steel wall of the soil cell, and most of it would be lost to the ambient air of $23^{\circ} \mathrm{C}$. The fiberglass insulation was inefficient to prevent heat loss from the soil cell. This phenomenon results in non-uniformity of heat flux and temperature distributions along the radial direction of the soil column, resulting in two-dimensional heat transfer. Therefore, design modification was made in order to address the above issues. 


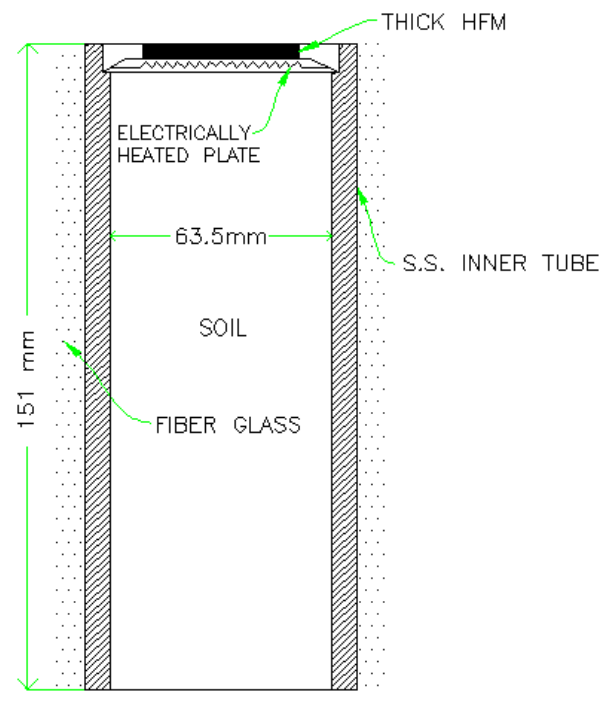

Fig. 2.1 Sketch of the preliminary design model

\subsection{Modified Model \#1}

The objective was to find an effective way to remedy the issues associated with the preliminary design. So, the idea of tube-in-tube with two separate layers of insulation (each layer of fiberglass with the same thickness, i.e. $25.4 \mathrm{~mm}$ ) was considered as a modification to the preliminary design, in order to minimize the heat loss. Fig. 2.2 illustrates the modified model of the preliminary design. In the modified model \#1, a larger stainless steel tube having inner diameter of $139 \mathrm{~mm}$ and thickness of $6.35 \mathrm{~mm}$ was introduced to surround the soil cell. The reason of considering larger stainless steel tube was basically to isolate the soil cell from the ambient air so that the heat loss from the soil cell to the ambient air can be minimal. After running the simulation in COMSOL for the modified model \#1, significant improvement was observed in energy balance analysis and the percentage difference between heat inflow and outflow was reduced to 59\%. However, this was not yet satisfying the design criteria, i.e. percent difference of less than $5 \%$. Hence, further modifications were needed to resolve the problem. 


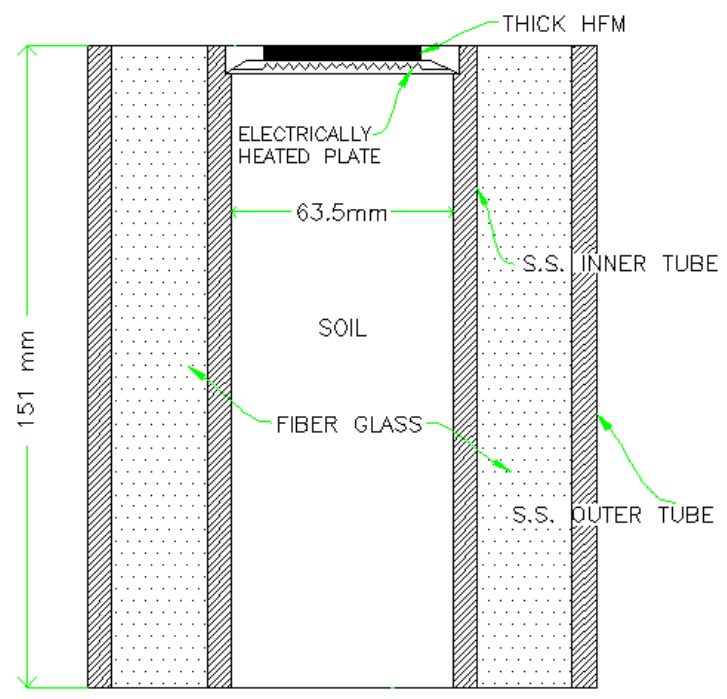

Fig. 2.2 Sketch of the modified model \#1

\subsection{Modified Model \#2}

The objective in this modification was to address the issue associated with modified model \#1. Fortunately, through an exhaustive search, a thin HFM with relatively high thermal conductivity $(3.33 \mathrm{~W} / \mathrm{m} \cdot \mathrm{K})$ was found and the manufacturer was able to specially coat the entire HFM to prevent moisture damage. Therefore, in the modified model \#2, the electrically-heated plate and high thermal resistive HFM were removed and replaced by the new thin HFM having relatively low thermal resistance. A thin HFM was also placed at the bottom of the soil column. The new thin HFMs have a thickness of $0.5 \mathrm{~mm}$, diameter of $63 \mathrm{~mm}$ and sensitivity of 15.4 $\mu \mathrm{V} / \mathrm{W} \cdot \mathrm{m}^{-2}$. Fig. 2.3 illustrates the sketch of this model. In this design, at both ends of the soil cell, a thin aluminum plate was placed in the circumferential recess of the stainless steel cylindrical wall and sealed with sealant to prevent moisture loss, while the thin HFM was sandwiched in between soil and the thin aluminum plate. After running COMSOL simulation of the modified model \#2, significant improvements were observed in both energy balance and heat flux variations over the radial direction. Fig. 2.4 presents the results of heat flux distribution over radial direction at two different heights in the soil column obtained from COMSOL simulation. From the results of simulation, it was observed that the highest difference in the radial heat flux distribution occurs at the upper regions of the soil column and it was found to be $2.2 \%$ difference for the worst case of $90^{\circ} \mathrm{C}$ hot plate and $75^{\circ} \mathrm{C}$ cold plate. For lower temperature levels, the difference would be lower due to lower heat loss. 


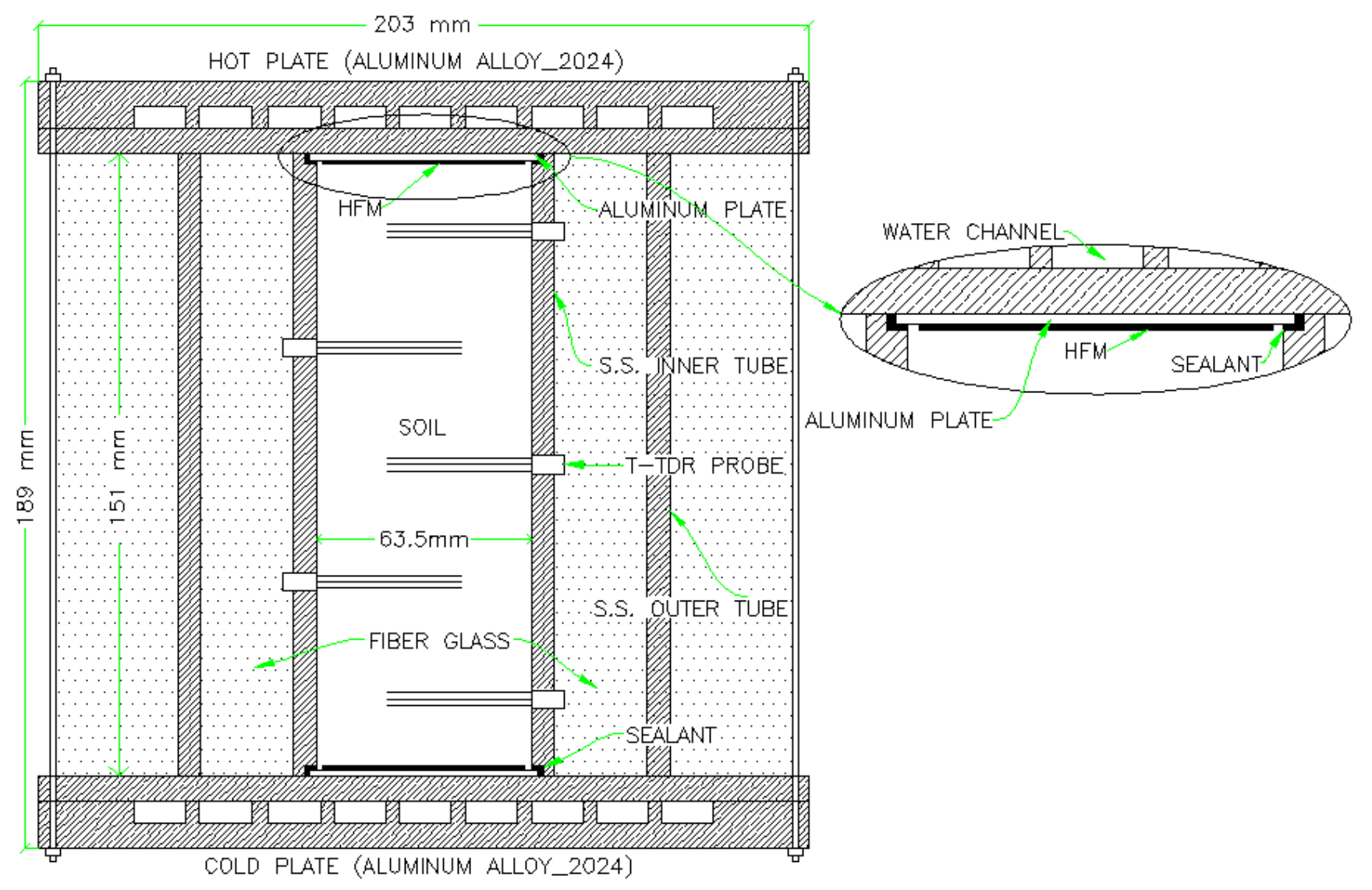

Fig. 2.3 Sketch of the modified model \#2 secured in between the hot and cold plates

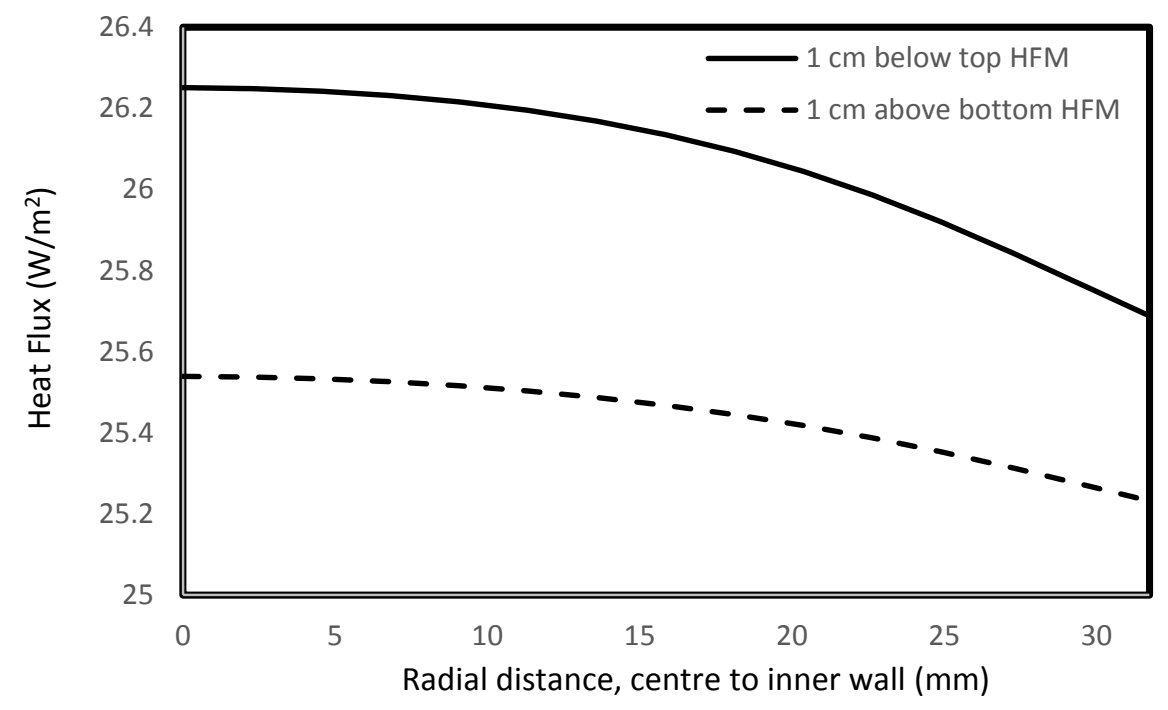

Fig. 2.4 Heat flux distribution over radial direction at two different heights in the soil column at the temperature level of $82.5^{\circ} \mathrm{C}$ and temperature difference of $15^{\circ} \mathrm{C}$. 
Fig. 2.5 illustrates the numerical model simulated in COMSOL. Three types of boundary conditions were applied, namely: temperature boundaries at the channels in the hot and cold plates ( $363 \mathrm{~K}$ and $348 \mathrm{~K}$, respectively), convection boundary at the outer surfaces of insulation with a convection coefficient of $6.44 \mathrm{~W} / \mathrm{m}^{2} \cdot \mathrm{K}$ and ambient temperature of $23^{\circ} \mathrm{C}$, and axisymmetric boundary at the centerline of the apparatus (Fig. 2.5(a) and (b)). Perfect thermal contacts between materials were assumed. Table 2.1 provides the material properties used in the COMSOL simulations. As shown in Fig. 2.5(c), finer meshes were applied in the regions where large temperature gradients exist, i.e. regions around the top and bottom of the soil cell.

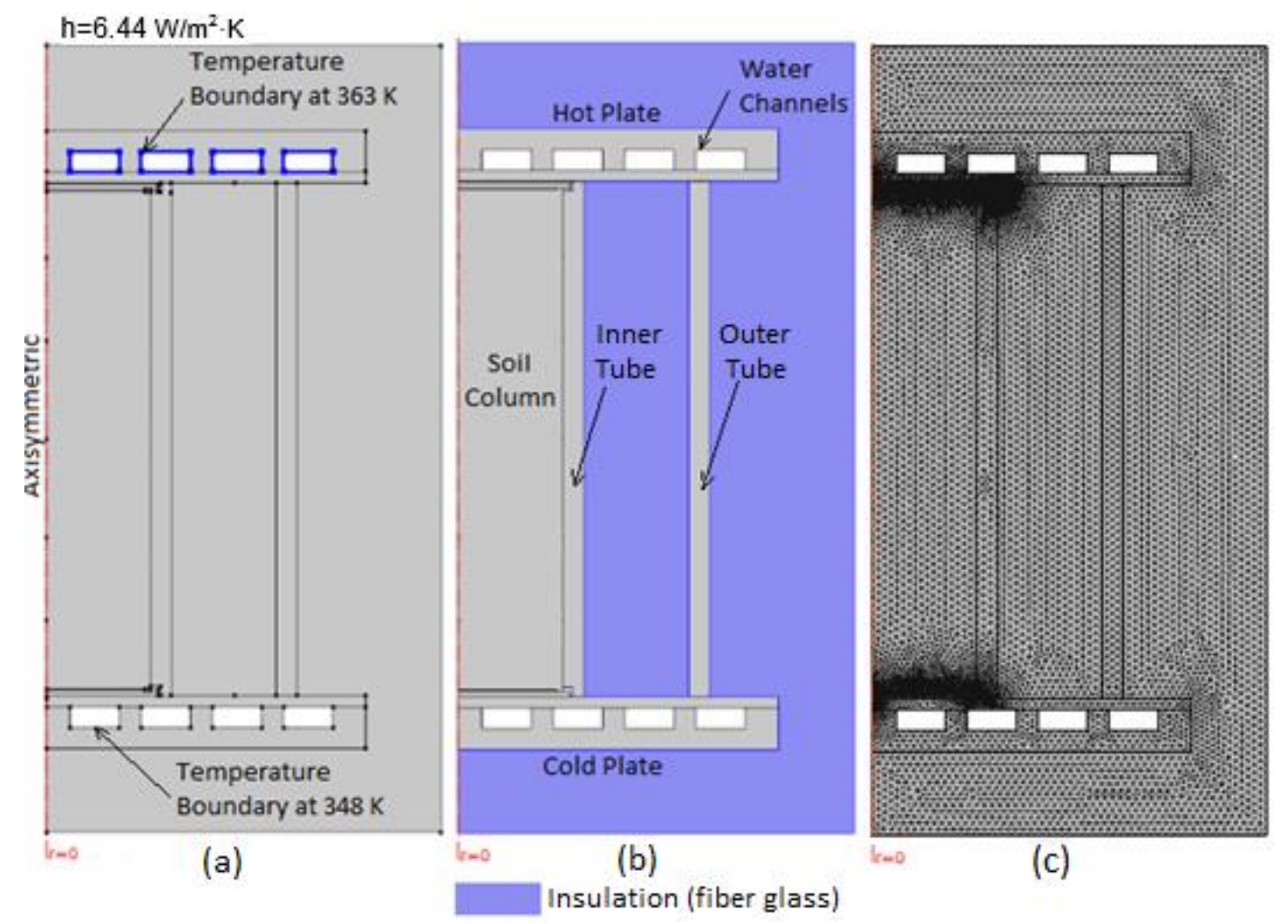

Fig. 2.5 Numerical model simulated in COMSOL: a) boundary conditions, b) materials, c) finiteelement mesh of the model

In Appendix C, Fig. C.1 demonstrates a constructed soil cell of modified model \#2 containing the wet soil when the cell is opened from the top side and when it is closed with aluminum thin plate. 
Table 2.1 Material properties used in COMSOL simulations

\begin{tabular}{|c|c|c|c|}
\hline & $\begin{array}{c}\rho \\
\left(\mathrm{kg} / \mathrm{m}^{3}\right)\end{array}$ & $\begin{array}{c}k \\
(\mathrm{~W} / \mathrm{m} \cdot \mathrm{K})\end{array}$ & $\begin{array}{c}C_{p} \\
(\mathrm{~J} / \mathrm{kg} \cdot \mathrm{K})\end{array}$ \\
\hline $\begin{array}{c}\text { Stainless Steel } \\
\text { (AISI 304) }\end{array}$ & 7900 & 14.9 & 477 \\
\hline $\begin{array}{c}\text { Aluminum } \\
\text { (Alloy 2024) }\end{array}$ & 2770 & 177 & 875 \\
\hline $\begin{array}{c}\text { PEEK } \\
\text { (Thermoplastic) }\end{array}$ & 1320 & 0.25 & 1200 \\
\hline $\begin{array}{c}\text { Dry Matilda soil } \\
\text { (Loamy sand) }\end{array}$ & 2706 & 0.26 & 2730 \\
\hline $\begin{array}{c}\text { Fiberglass } \\
\text { (Insulation) }\end{array}$ & 10 & 0.04 & 700 \\
\hline $\begin{array}{c}\text { Thin HFM } \\
\text { (Multi-layer }{ }^{4} \text { ) }\end{array}$ & 1100 & 3.33 & 2010 \\
\hline
\end{tabular}

\subsubsection{Material Selection for the Inner and Outer tubes}

COMSOL simulations for the modified model \#2 included simulations of three different types of the materials used for the inner and the outer tubes, in order to examine the best material for satisfying the design criteria of one-dimensional heat transfer. The three materials considered in this study were stainless steel 304, aluminum alloy 2024 and PEEK (high thermal resistance plastic). Their properties are given in Table 2.1. From the simulations, it was observed that stainless steel tubes are the best. Fig. 2.6 illustrates the results of temperature variations along the centerline of the soil cell for the modified model \#2 using the above three materials. It can be seen that the worst result was obtained for PEEK since the temperature variation along the centerline of the soil cell is clearly nonlinear. This failed to meet the design criterion of one-dimensional heat transfer along the soil cell. In comparison, from Fig. 2.6, it can be observed that the best result was obtained for stainless steel since the temperature variation is the closest to linear. Fig. 2.7 illustrates heat flux arrows and isotherms at the axisymmetric view of the apparatus from COMSOL

\footnotetext{
${ }^{4}$ Several layers including electrical insulated materials and metal
} 
simulation for the modified model \#2. The results show that in the case of stainless steel higher heat flux uniformity over the radial direction along the soil cell can be obtained. Hence, it can be argued that stainless steel was the most suitable selection to be used for the inner and outer tubes. In conclusion, since the results of numerical studies satisfied the design requirements, the fundamental design of an apparatus was approved and construction of apparatus was implemented according to the numerically approved design.

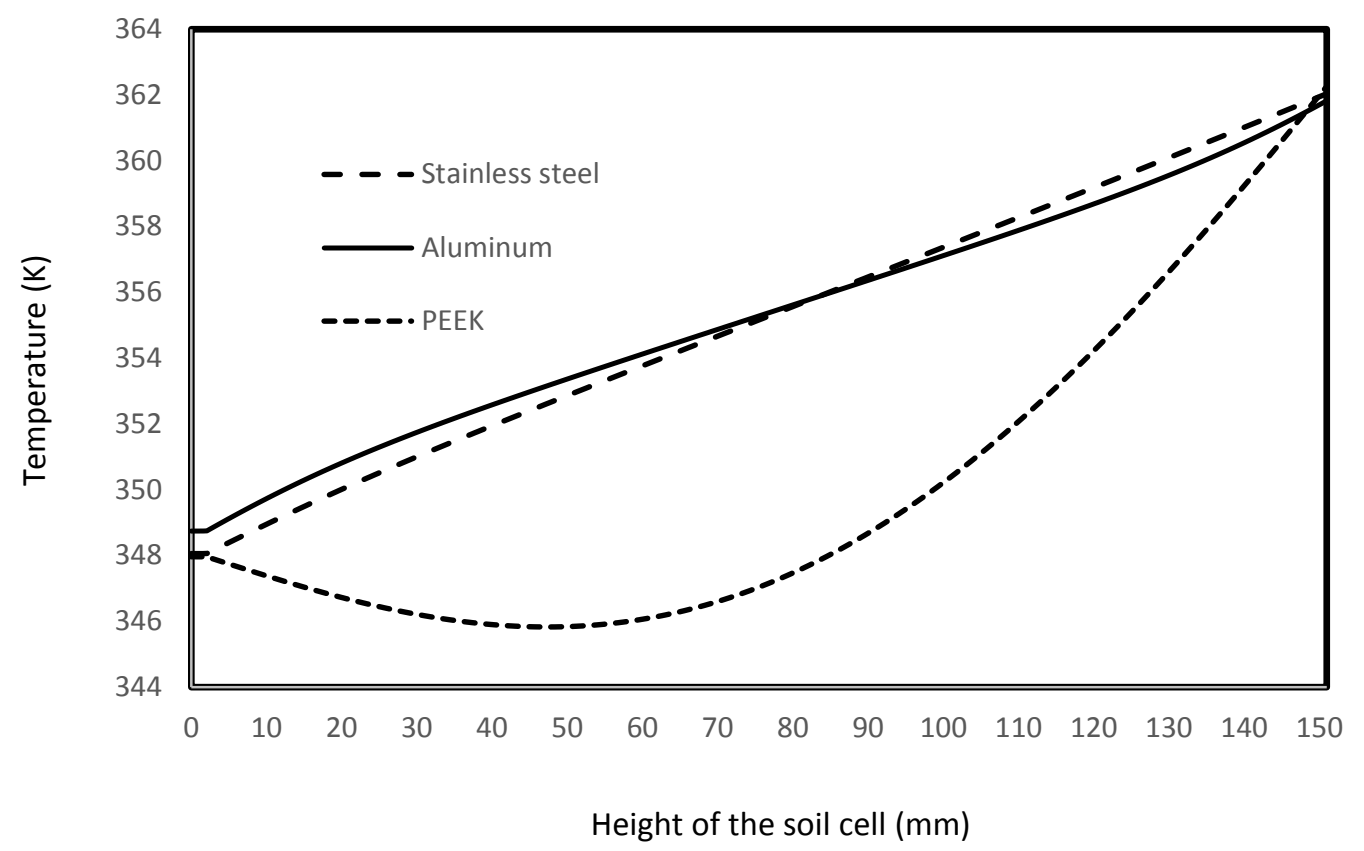

Fig. 2.6 COMSOL results of the temperature variations along the centerline of the soil cell for the modified model \#2 with three different tube materials 


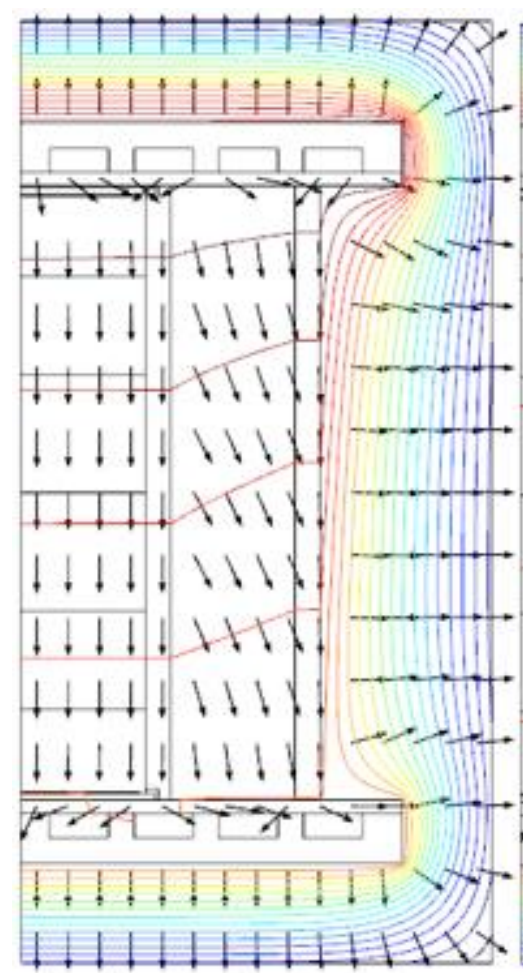

a) Stainless Steel

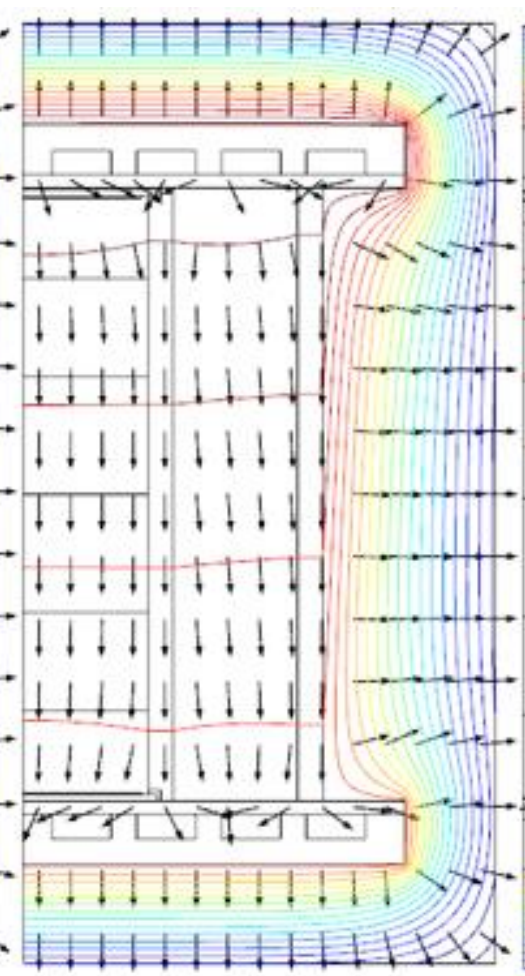

b) Aluminum

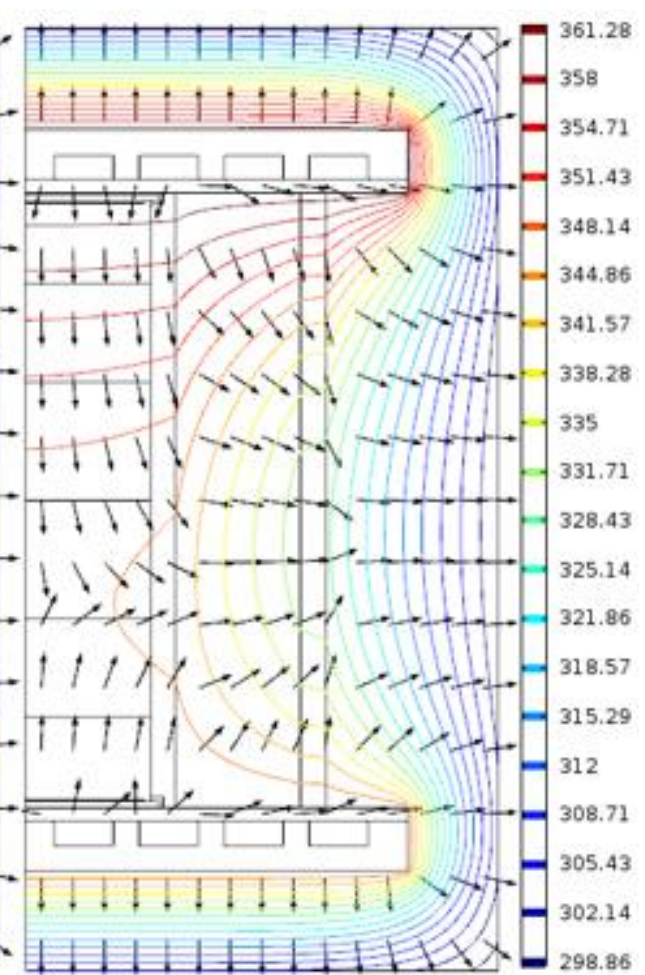

c) PEEK

(K)

Fig. 2.7 Views of heat flux vectors and isotherms of the apparatus from COMSOL simulation for the modified model \#2 with three different tube materials: a) stainless steel 304, b) aluminum alloy 2024 and c) PEEK

\subsection{Number of T-TDR Probes}

The maximum number of T-TDR probes to be used in the soil cell was required to be analyzed. In order to have maximum number of temperature readings along the soil column, the probes were inserted horizontally one after another into the soil column with their three needles aligned vertically, as shown in Fig. 2.3. The distance between the two probes was determined based on TDR interference and heat pulse effects. In this study, according to the results of the experimental evaluation of the interference of TDR probes (which will be presented in Chapter 5), the minimum distance between two adjacent TDR probes was to be about $20 \mathrm{~mm}$ apart so that the effect of interference would become negligible. Since the probes were to produce simultaneous heat pulses, the generated heat pulse of a probe could travel to adjacent probes, affecting temperature readings of the other probes and thus resulting in inaccurate measurements. Therefore, to avoid the effect of the heat pulse of one probe on another, it is critical to estimate how far the heat travels after it was generated by the middle resistance heating needle of the probe. According 
to Bristow et al. (1994), the maximum radial distance that the heat travels from the heating source can be expressed by the following relation.

$d=2 \sqrt{D_{a} t}$

where $d$ is the estimated travel distance of the heat $(\mathrm{m}), D_{a}$ is thermal diffusivity of the soil $\left(\mathrm{m}^{2} / \mathrm{s}\right)$ and $t$ is the elapsed time since the heat was started to be generated (s). Using a typical value of thermal diffusivity of $6.7 \times 10^{-7} \mathrm{~m}^{2} / \mathrm{s}$ for a wet Matilda soil at $50 \%$ saturation degree and a typical measurement period of 120 seconds, the estimated travel distance of the heat was found to be 18 $\mathrm{mm}$. Therefore, to be on the safe side, it was decided to have five T-TDR probes and allow them to be spaced equally at a distance of about $29 \mathrm{~mm}$ between probe centers or $23 \mathrm{~mm}$ between the middle resistance heating needle and an adjacent thermocouple needle of another probe. Appendix B.3 presents engineering CAD drawing of the soil cell for the modified model \#2.

\subsection{Design of the Hot and Cold Plates}

The design objective was to maintain a uniform desired temperature throughout the entire front surface of the hot or cold plate. The hot or cold plate consists of two separate plates. One plate would have a grooved channel for circulating water and the other would serve as a cover for the grooved plate. The dimensions of the plates were selected to be $203 \times 203 \times 12.7 \mathrm{~mm}$ for the grooved plate and $203 \times 203 \times 6.35 \mathrm{~mm}$ for the cover plate. Appendix B.2 presents engineering drawing for the grooved plate. The plates were to be maintained at isothermal conditions by circulating water from two thermally-controlled baths. The design principle was established based on the criterion that the change of circulating water temperature through the plate has to be not greater than $0.1^{\circ} \mathrm{C}$. In the beginning of this experimental study, the plan was to conduct experiments based on the temperature difference $(\Delta T)$ between hot and cold plates from $5^{\circ} \mathrm{C}$ to $65^{\circ} \mathrm{C}$; therefore, selecting $0.1^{\circ} \mathrm{C}$ change would only cause $2 \%$ uncertainty in the worst case scenario, i.e. $\Delta T=5^{\circ} \mathrm{C}$, which was acceptable. In the process of selecting the best material, aluminum alloy 2024 and stainless steel 304 were considered and evaluated based on their thermal capacitance $^{5}$, yield strength, easy to machine and also availability in the metal market. Aluminum alloy 2024 with yield strength of $324 \mathrm{MPa}$ (47000 psi) and thermal capacitance of $2424 \mathrm{~kJ} / \mathrm{m}^{3} \cdot \mathrm{K}$

\footnotetext{
${ }^{5}$ Thermal capacitance or thermal mass refers to the ability of the body to store thermal energy.
} 
was selected over stainless steel 304 with yield strength of $207 \mathrm{MPa}$ (30000 psi) and thermal capacitance of $3768 \mathrm{~kJ} / \mathrm{m}^{3} \cdot \mathrm{K}$, since it has lower thermal capacitance and also it is easier to machine. In fact, the lower the thermal capacitance, the lower the ability of the material to store thermal energy so that this results in the faster distribution of heat throughout the material.

\subsubsection{CFD Modeling of the Channel Grooved Plates}

The objective was to design the channel grooved on aluminum alloy 2024 plate in such a way that the required mass flow rate of circulating water provides uniform temperature distribution throughout the entire surface of the aluminum plate. Based on the design principle, for a maximum temperature difference of $0.1^{\circ} \mathrm{C}$ between inlet and outlet at steady state conditions, the total heat transfer rate is given by

$Q=\dot{m} c_{w} \Delta T_{w}$

where $\dot{m}$ is the mass flow rate of water $(\mathrm{kg} / \mathrm{s}), c_{w}$ is specific heat of water $(\mathrm{J} / \mathrm{kg} \cdot \mathrm{K}), \Delta T_{w}$ is the temperature change of circulating water from inlet to outlet $\left(\Delta T_{w}=0.1^{\circ} \mathrm{C}\right)$, and $Q$ is total heat transfer from the hot plate to the cold plate via the two stainless steel tubes, the soil and the insulation. For the worst case scenario, i.e. $90^{\circ} \mathrm{C}$ hot plate and $10^{\circ} \mathrm{C}$ cold plate, the highest heat transfer was found from COMSOL simulation to be about $40 \mathrm{~W}$. Therefore, the required mass flow rate of circulating water was determined using Eq. (2.2) to be about $0.1 \mathrm{~kg} / \mathrm{s}$. By using the required mass flow rate of water for the most crucial scenario, a computational fluid dynamics (CFD) model of the three-dimensional grooved channel was simulated using k- $\varepsilon$ turbulence model in COMSOL. In the CFD simulation, the objective was to find the pressure drop through the grooved channel for the required mass flow rate of circulating water. The simulated pressure drop from the CFD was then compared with the pump performance curve of the thermally-controlled baths to ensure that the pump could provide the required circulation of water in the closed loop system. Several designs of the grooved channels were studied. Figs. 2.8 and 2.9 illustrate the layout of one of the best channel grooved on an aluminum plate and its general meshed structure. The inlet and outlet of the channel has circular cross sections having diameter of $6.35 \mathrm{~mm}$. The best rectangular cross section of the channel was found to be $15 \times 6.35 \mathrm{~mm}$, which would allow a higher mass flow rate of circulating water than the required mass flow rate as a safety precaution. From 
the CFD results for $0.19 \mathrm{~kg} / \mathrm{s}$ of the mass flow rate of water, the pressure drop from the inlet to the outlet was found to be about 0.34 bar or $34 \mathrm{kPa}$. Also, the pressure drop due to the rubber pipe from the thermal baths to the hot and cold plates was found to be 0.26 bar or $26 \mathrm{kPa}$. Therefore, the total pressure drop was found to be 0.6 bar or $60 \mathrm{kPa}$. The operating point was found to be possible on the pump performance curve for pump level 8 setting of the thermally-controlled bath ${ }^{6}$.

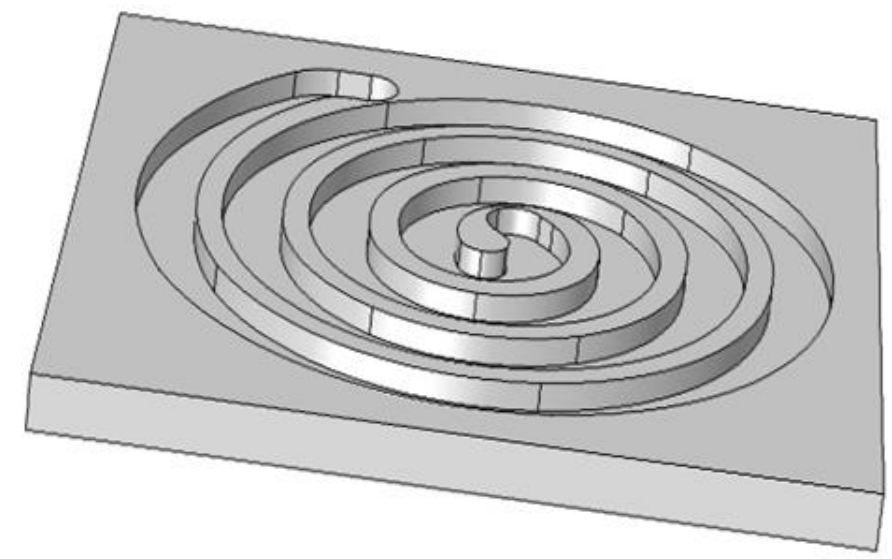

Fig. 2.8 Depiction of channel grooved on aluminum alloy 2024 plate

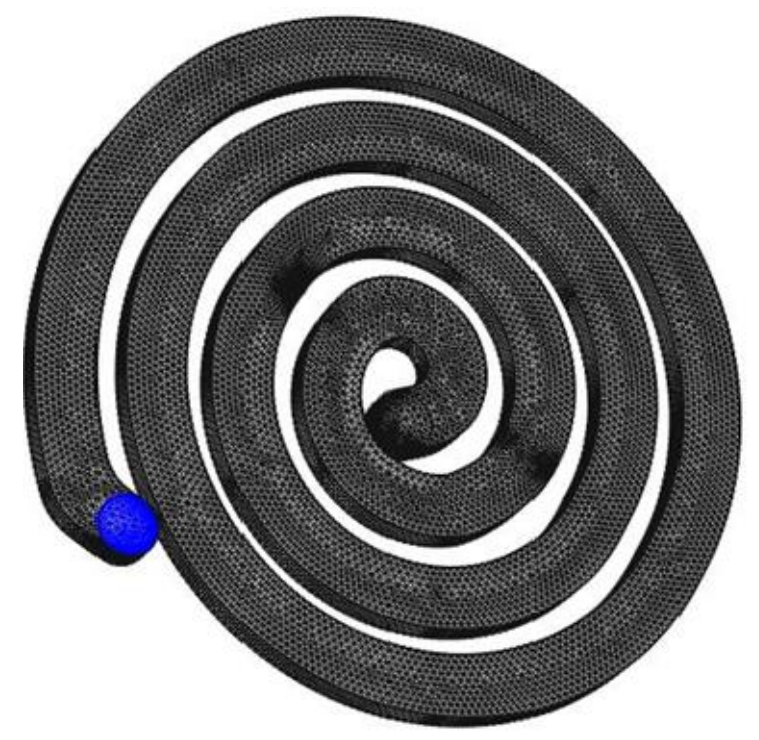

Fig. 2.9 3D finite-element mesh structure of the water channel on aluminum plate

${ }^{6}$ LAUDA PROLINE RP1845 


\subsection{Mesh Considerations of the Numerical Model}

To have reliable computations for the model simulation, specific considerations are required when generating the mesh. One of the methods used in mesh sensitivity assessment is the technique of doubling. When using the doubling technique, in order to obtain appropriate mesh resolution, the number of the nodes in the first mesh would double up in the second mesh to examine how the results change. This method continues until no significant change in results, i.e. maximum 5\% change, is observed. The method used in this study consists of two steps. The first step was to simulate the model with arbitrary coarse mesh and the second step was to improve the mesh resolution and re-simulate the model. By comparing the results from the two simulations, according to the required confidence level, it can be concluded whether higher mesh resolution is needed. In this study, mesh resolution was defined using COMSOL mesh custom option so that mesh resolution could be adjusted manually. The required mesh resolution throughout the geometry of the model was not uniformly defined by COMSOL since the regions near the wall or sharp edges need to have higher mesh resolution due to the higher gradients. In this study, for 2D axisymmetric model, the final and finest grid had maximum and minimum element sizes of 0.5025 $\mathrm{mm}$ and $1.005 \times 10^{-3} \mathrm{~mm}$, respectively, with a total number of 293435 domain elements. For 3D space model, i.e. CFD simulation of grooved channel, the grid had maximum and minimum element sizes of $2.5 \mathrm{~mm}$ and $0.5 \mathrm{~mm}$, respectively, with a total number of 435931 domain elements.

\subsection{Experimental Problem of Modified Model \#2}

Although the results from the numerical simulations of the modified model \#2 were satisfying, problematic consequences were observed after the model was constructed and tested using dry soil. The results showed significant discrepancies, as high as $11 \%$, between the heat flows entering from the top and exiting from the bottom of the soil cell. After some investigations, it was found that the modified model \#2 has two physical problems. First the thin aluminum plates, which were used to cap the soil cell by sealing them in the recessed portions of the stainless steel cylinder, caused non-uniformity of heat entering into and departing from the soil due to high thermal conductivity of aluminum conducting significant amount of heat to the stainless steel cylinder. Second the contact regions between soil and thin HFMs were not leveled consistently 
due to the recessed portions of stainless steel cylinder. The uneven soil surfaces would cause poor contact between soil and thin HFMs. In addition, the uneven aluminum plates also cause contact problem with the hot and cold plates. Other than the two physical problems, there was one drawback about sealing the aluminum plates in the recessed portions of the stainless steel cylinder with a gasket sealant to prevent moisture loss from the soil cell. That is, due to the stickiness of the sealant, it was very difficult to remove the aluminum plates and the sealant from the recesses after an experiment.

\subsection{Modified Model \#3}

In order to solve the two physical problems and drawback of the modified model \#2, the following objectives were considered when the new soil cell model was being designed:

1- To eliminate the difference in thermal conductivities of the aluminum plates and the stainless steel cylinder for the soil cell, the aluminum plates must be replaced by stainless steel plates.

2- To eliminate the soil surface level problem, which causes poor contact between the soil and the thin HFMs, there must be no recessed portions of stainless steel cylinder.

3- To eliminate the problems associated with using the gasket sealant for prevention of moisture loss, a cap which can be tightened with screws would be a more effective way to seal the soil cell.

Fig. 2.10 illustrates the layout of modified model \#3. Appendix B.5 presents engineering drawing of the modified model \#3. In new design, the height of the inner tube was reduced to 147.9 $\mathrm{mm}$. Also, the recessed portions were eliminated and thin aluminum plates were replaced by thin stainless steel plates having a thickness of $1.55 \mathrm{~mm}$ each and diameter of $76.2 \mathrm{~mm}$, which would completely cover the top and bottom of the soil cell. In addition, the thin stainless steel plates were secured to the top and bottom sides of stainless steel cylinder with six flat head stainless steel screws on each side, each having total length of $4.65 \mathrm{~mm}$. The secured attachment of thin stainless steel plates and stainless steel cylinder helped not only to prevent moisture loss, but also to enhance the capability of the soil cell to withstand the water vapor pressure as a result of high-temperature conditions. Since the recessed portions were eliminated, the surface of the soil could be properly 
leveled with the top of stainless steel cylinder using a straight-edge ruler. With all these modifications, the above-mentioned objectives were addressed. Fig. 2.11 shows the numerical results of the modified model \#3. Comparing to Fig. 2.7(a), the modified model \#3 has better result, especially near the bottom HFM. In Appendix C, Fig. C.2 shows a constructed soil cell of modified model \#3 containing the wet soil when the cell is opened from the top side and when it is closed securely with stainless steel thin plate. In Appendix C, Fig. C.3 demonstrates a constructed soil cell of modified model \#3, with inserted in-house made T-TDR probes, surrounded by the outer stainless steel tube. In Appendix C, Fig. C.4 (a) shows a constructed soil cell of modified model \#3 with surrounded fiber glass insulation and Figs. C.4 (b) and C.4 (c) illustrate an apparatus, sandwiched between two differentially heated plates, without and with outer surrounded fiber glass insulation, respectively.

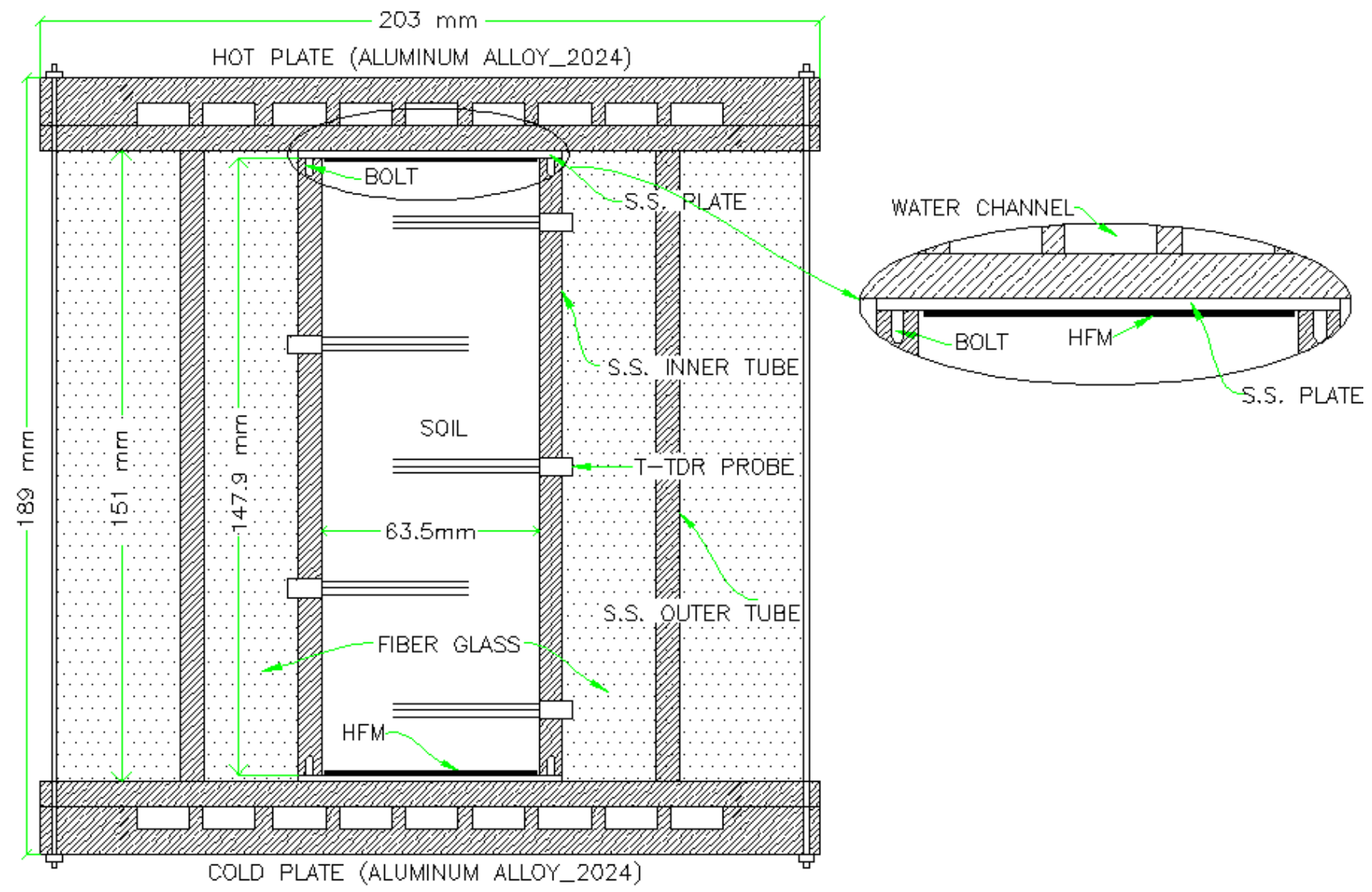

Fig. 2.10 Sketch of cross section of the modified model \#3 


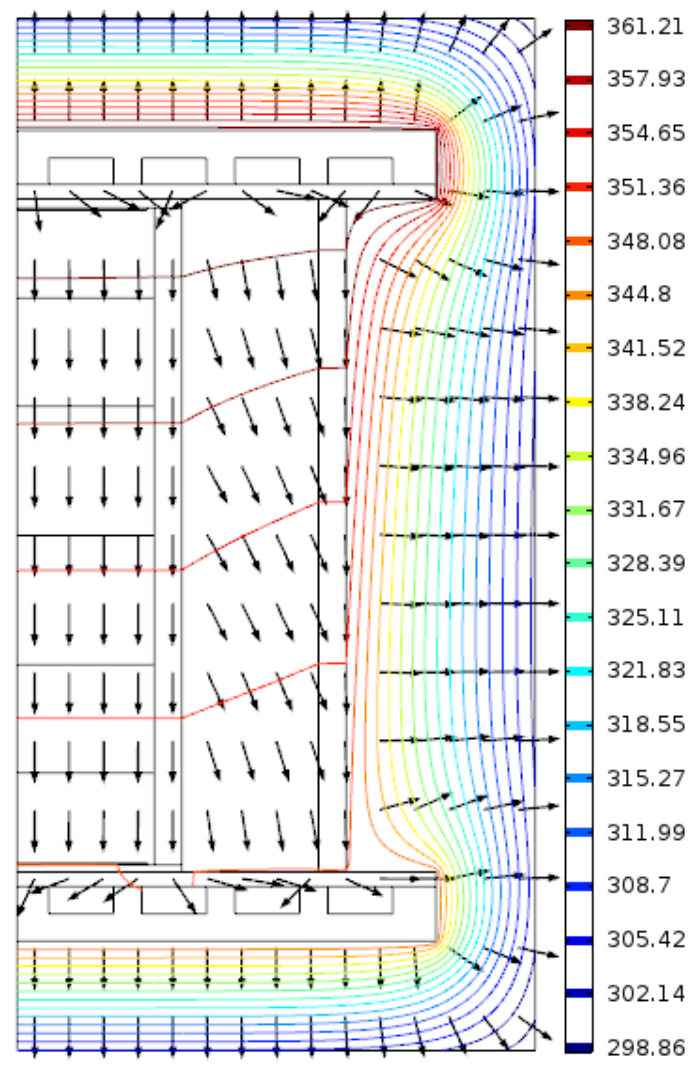

(K)

Fig. 2.11 Views of heat flux vectors and isotherms of the apparatus from COMSOL simulation for the modified model \#3

\subsection{Summary}

The design of an experimental apparatus was established based on the criterion of having one-dimensional heat flow within a soil cell filled with a dry soil. For a cylindrical soil cell with an axial axis along its centre, the existence of one-dimensional heat flow within a soil cell can be indicated by two conditions. If both ends of the cylindrical soil cell are differentially heated, the first indication-in an ideal steady-state condition-was to have the same inflow and outflow of heat transfer rates through the soil cell, and the second indication-in an ideal steady-state condition-was to have uniform heat flux distribution in the radial direction at any cross section of the cell. In the present study, these conditions were considered to be met when the variations are within $\pm 5 \%$. In order to provide differential heating, the soil cell would be sandwiched between two differentially heated plates made from aluminum alloy 2024. The design principle of the heated plates was to have uniform temperature distribution over the entire surface areas of the 
plates. In this study, with respect to the design principle, the general integrity and reliability of the models were numerically assessed using COMSOL, based on realistic geometries and boundary conditions.

The results of the preliminary design simulations showed that the differences of energy balance, i.e. percent difference between average heat fluxes entering on top of the soil column and exiting from the bottom, remain as high as $218 \%$. Since the dry soil has relatively low thermal conductivity compared to aluminum and stainless steel, most of the heat would bypass from the soil to the stainless steel wall of the soil cell, and most of it would be lost to the ambient air of $23^{\circ} \mathrm{C}$. The fiberglass insulation was inefficient to prevent heat loss from the soil cell. This phenomenon results in non-uniformity of heat flux and temperature distributions along the radial direction of the soil column, resulting in two-dimensional heat transfer. Therefore, design modification was made in order to address the above issues.

In modified model \#1 the idea of tube-in-tube with two separate layers of insulation was considered as a modification to the preliminary design, in order to minimize the heat loss. The results of the modified model \#1 numerical simulations showed significant improvement in energy balance analysis. Indeed the percentage difference between heat inflow and outflow was reduced to $59 \%$. However, this was not yet satisfying the design criteria.

In the modified model \#2, the electrically-heated plate and high thermal resistive HFM were removed and replaced by the new thin HFM. A thin HFM was also placed at the bottom of the soil column. In this design, at both ends of the soil cell, a thin aluminum plate was placed in the circumferential recess of the stainless steel cylindrical wall and sealed with sealant to prevent moisture loss, while the thin HFM was sandwiched in between soil and the thin aluminum plate. The results of the modified model \#2 numerical simulations showed significant improvements in both energy balance and heat flux variations over the radial direction. From the results of simulation, it was observed that the highest difference in the radial heat flux distribution occurs at the upper regions of the soil column and it was found to be $2.2 \%$ difference for the worst case of $90^{\circ} \mathrm{C}$ hot plate and $75^{\circ} \mathrm{C}$ cold plate. 
Although the results from the numerical simulations were satisfying, issues were found after the modified model \#2 was constructed and tested using dry soil. The results showed significant discrepancies, as high as $11 \%$, between the heat flows entering from the top and exiting from the bottom of the soil cell. It was found that the modified model \#2 has two physical problems. First the thin aluminum plates caused non-uniformity of heat entering into and departing from the soil. Second the contact regions between soil and thin HFMs were not leveled consistently. The uneven soil surfaces would cause poor contact between soil and thin HFMs. Other than the two physical problems, there was one drawback about sealing the aluminum plates in the recessed portions of the stainless steel cylinder with a gasket sealant to prevent moisture loss from the soil cell.

Therefore, in designing the modified model \#3 the modifications were made in order to address the problems associated with the modified model \#2. First to eliminate the difference in thermal conductivities of the aluminum plates and the stainless steel cylinder for the modified model \#2 soil cell, the aluminum plates were replaced by stainless steel plates. Second to eliminate the soil surface level problem, the recessed portions of stainless steel cylinder were not considered anymore and third to eliminate the problems associated with using the gasket sealant for prevention of moisture loss, stainless steel caps were tightened with screws to seal the soil cell more effectively. 


\section{CHAPTER 3}

\section{EXPERIMENTAL TECHNIQUES}

\subsection{Introduction}

The methodology used in this experimental study is called Thermo-Time Domain Reflectometry (T-TDR) which is basically a combination of two different techniques: TDR and Heat pulse. T-TDR is a technique which is used to measure soil volumetric water content $(\theta)$, temperature $(T)$ and thermal properties such as volumetric heat capacity $(C)$, thermal diffusivity $\left(D_{a}\right)$ and thermal conductivity $(k)$. T-TDR probes combine the technologies of TDR probe and heat pulse probe into one single probe with three needles. The volumetric water content of the soil can be determined using both TDR and heat pulse techniques. Heat pulse is produced from the resistance heater wire in the central needle of the probe which is connected to a DC power supply via relay controller. The relay controller is a device which opens or closes the power circuit to generate a heat pulse from the central needle as signalled by the data-logger. The generated heat

pulse will transfer radially outward toward the two side needles which each contain a thermocouple. Using the TDR technique the water content can be measured by sending short electromagnetic wave through the soil via the TDR probe. The TDR probe is the measuring device of the TDR system and is implanted into the soil. The TDR probe is basically a wave guide on the end of coaxial cable. In this method the travel time of an electromagnetic wave pulse through a wave guide is being measured. Assume that the wave guide is immersed completely in a wet soil, as electromagnetic wave propagates through the soil portion of an incident electromagnetic wave is reflected at the start of the probe due to the impedance difference between coaxial cable and stainless steel probe. The change in the probe impedance alter the shape of reflected electromagnetic wave pulse. In fact the form of the reflected pulse contains information to find moisture content of the soil. The impedance is associated with the size and spacing of wave guides of the probe and it is inversely proportional to the dielectric constant of the soil. The velocity of the electromagnetic wave traveling though the soil is a direct function of the soil dielectric constant. Since the change in the water content of the soil would significantly impact its dielectric constant, therefore, water content can be determined by computing its dielectric constant in TDR system. The soil thermal properties can be measured by sending a heat pulse through the soil via the heat pulse probe. 
In this study, the experimental tests were divided into three main stages. The first stage was a preliminary evaluation of the capability of the in-house made TDR probes and the TDR system, as a whole, to determine volumetric water content of soils, as well as evaluation of the effects of the probe interference using three different soils. The results of this evaluation provided a general assessment of the measurement uncertainties of the TDR system, as well as a general design guideline about a proper spacing between TDR probes for minimizing the interference effects. The second stage was a preliminary evaluation of the capability of the apparatus and soil cell to attain the design criteria of one-dimensional heat transfer in the soil cell. The results of this evaluation led to a final design of the soil cell (modified model \#3). The third stage of the experimental tests was a study of the heat and moisture transfer in a soil column at high temperature conditions. In this last stage of study, water contents were determined using the heat pulse technique. In the following subsections, the techniques applied in this study to determine soil volumetric water content and thermal properties are presented.

\subsection{Time Domain Reflectometry}

In early 1980, Topp et al. (1980) found travel times in TDR probes by applying tangent line method for the reflected waveforms created by electromagnetic signals. The shape of the waveform is mainly dependent on the length of the probe, soil water content and soil bulk density. During the past decade, the TDR method has become a standard technique for determining moisture content of the soil because it provides fast sensing of soil volumetric water content while soil disturbance is minimal and spatial resolution is greatly reliable (Robinson et al. 2003). Capturing electromagnetic waveforms using the TDR method is necessary in soil water content and soil conductivity studies. It is more common to use longer TDR probes having the probe length of $15 \mathrm{~cm}$ or longer since they are more accurate for measuring water content. TDR probes used in this study are designed to be relatively short so that they can be utilized in the applications with limited soil volume. However, this requires more sophisticated waveform analysis for determining water content of the soil. The reason is that the reflected pulse through the entire waveform is quite small to be identified. So it is best to capture the reflected waveform entirely and apply techniques such as 'tangent-line method' or 'second-order bounded mean oscillation (BMO)' in order to analyze the reflected pulse on a plot of reflection coefficient versus distance, as shown in Fig. 3.1. In Fig. 3.1, reflection coefficients refer to the points where signal propagation reflection occurs 
due to impedance difference between coaxial cable and stainless steel needle. Distance refers to the electromagnetic length of the probe which is characterized on the TDR result screen. The accuracy of the tangent-line method is limited for short TDR probes; however, the second-order BMO is capable to give more plausible results for some waveforms (Wang et al. 2014). Software based on an algorithm of the second-order BMO has been developed (Schwartz et al. 2013) and is readily available from the Internet (http://soilphysics.agron.iastate.edu). The software was employed in this study for determining reflection positions of the waveforms and calculating water content for TDR probe interference study. The algorithm undertakes the reflection positions corresponding to the local maxima of the waveform. So the reflection position can be identified by the algorithm if the local maxima are selected properly. The main drawback of using secondorder BMO is that it is challenging to correctly set a default threshold for all TDR waveforms. Therefore, manual adjustment may be needed to find a right threshold (Wang et al. 2014).

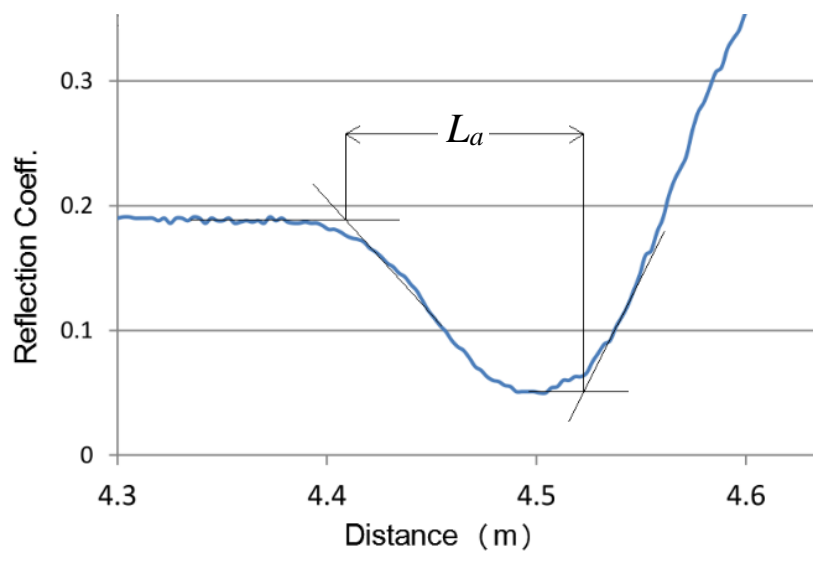

Fig. 3.1 An example of tangent line method used to find apparent probe length $\left(L_{a}\right)$ from the reflected waveform

\subsubsection{Theory}

To simply explain the theory of TDR waveform analysis, Fig. 3.1 illustrates an example of the tangent-line method to find the distance between the reflection points on a typical electromagnetic waveform recorded in the present study. In this method finding initial and final reflection points acquires the intersections of tangent lines with a horizontal line for the first and second slopes. The round trip time between these reflected waves from the beginning to the end of the probe can be described as (Fellner-Feldegg 1969) 
$t=2 L K^{0.5} / v$

where $t$ is a round trip time between the reflected waves (s), $v$ is the velocity of electromagnetic waves in free space $\left(3 \times 10^{8} \mathrm{~m} \cdot \mathrm{s}^{-1}\right), L$ is the physical length of probe needle $(L \approx 0.05 \mathrm{~m})$, and $K$ is the dielectric constant of soil. Eq. (3.1) can be rearranged as

$K=(v t / 2 L)^{2}$

Generally for most common used coaxial cables the term $v t / 2$ is equal to the apparent length of the probe $\left(L_{a}\right)$ (Baker et al. 1990) so that dielectric constant can be expressed as

$K=\left(L_{a} / L\right)^{2}$

Topp et al. (1980) found an empirical equation to calculate volumetric water content for homogeneous soil which is defined as

$\theta=\left(4.3 \times 10^{-6}\right) K^{3}-\left(5.5 \times 10^{-4}\right) K^{2}+\left(2.92 \times 10^{-2}\right) K-\left(5.3 \times 10^{-2}\right)$

The spatial resolution of the TDR measurement is considered as a distribution of the propagated electromagnetic waveform within the wave guide surrounding volume. Baker and Lascano (1989) found the spatial resolution of TDR technique using experimental procedures. According to their studies, it was determined that the spatial resolution of TDR measurement is limited to a region with a cross section area of about $0.001 \mathrm{~m}^{2}$. Laboratory studies by Ren et al. (2005) showed that their T-TDR probe has the spatial resolution of an approximate radius of 11 $\mathrm{mm}$ about the wave guide. In this study, it was determined that the spatial resolution of TDR measurement is a radius of about $11 \mathrm{~mm}$ surrounding the wave guide ${ }^{7}$. Therefore, spatial resolution of TDR technique for measuring soil water content was found to be $1.52 \times 10^{-5} \mathrm{~m}^{3}$ surrounding the central needle of the T-TDR probe.

\subsubsection{TDR System}

The process of finding volumetric water content of the soil using TDR system requires two main steps. The first step is to find the entire waveform as a results of electromagnetic signal's

\footnotetext{
${ }^{7}$ Refer to Chapter 5 for more detail
} 
reflection for every measurement and the second step is to accurately analyze the waveform in order to estimate soil water content. In this study Campbell Scientific's Time Domain Reflectometry (TDR) system was employed to execute TDR technique. The system is comprised of the TDR100 Time Domain Reflectometer device, CR1000 data-logger, SDMX50 coaxial multiplexers, PC-TDR software and Logger-net software which supports data-logger programming, data transfer and data processing functions. The TDR100 device produces short electromagnetic pulse that is applied to a coaxial cable of the probe and captures the resulting reflection waveform as digital data for analysis. The data-logger CR1000 is a device which can be programmed for automatic control of the TDR100 and SDMX50 multiplexers. In this study, Campbell Scientific's PC-TDR software was used to characterize all the constructed TDR probes. The characterized probes are to be defined to the TDR system via Logger-net software by the means of CRBasic programing code. In order to properly characterize the probes, two important parameters need to be determined. These parameters are known as 'cable length' and 'window length'. The cable length parameter specifies the cable length of the coaxial cable and window length identifies the length of the waveform to be collected. The waveform initiates at the cable length and ends at the summation of cable length and window length. These parameters are to be determined by PC-TDR software. Using PC-TDR the user can adjust the cable length and window length so that the reflection pulse though the entire waveform can be displayed clearly. The following steps were performed in order to determine cable and window lengths of all the probes:

1- Disconnect connections of C1, C2 and C3 ports between data-logger and TDR100

2- Submerge each probe individually into de-ionized water (de-ionized water has minimum ions which can cause the interference with electromagnetic signals).

3- Connect all the probes to SDMX50 multiplexer channels.

4- Set the SDM address at 0 on TDR100 and 1 on SDMX50.

5- Set the lengths 0 and $25 \mathrm{~m}$ in $\mathrm{X}$ axis and -2 and $2 \mathrm{~m}$ in $\mathrm{Y}$ axis.

6- Click on "get waveform" to see the entire wave form.

7- Identify reflected pulse through the entire waveform.

8- Adjust X and Y coordinates so the waveform will be similar to the one in Fig. 3.2.

9- Record cable and window lengths to be used in CRBasic programing code. 
Depending on consistency of the probes construction, cable and window lengths may vary to some extent. In this study, for all the in-house made probes, cable and window lengths were determined to be respectively $4.3 \mathrm{~m}$ and $0.7 \mathrm{~m}$. Since the in-house made T-TDR probe was much shorter than any typical TDR probe sold commercially ( $4 \mathrm{~cm}$ vs. $15 \mathrm{~cm}$ or longer), the technical staffs from Campbell Scientific were unable to provide any assistance in characterizing the probe. Through a long trial-and-error process, the probe was finally characterized successfully.

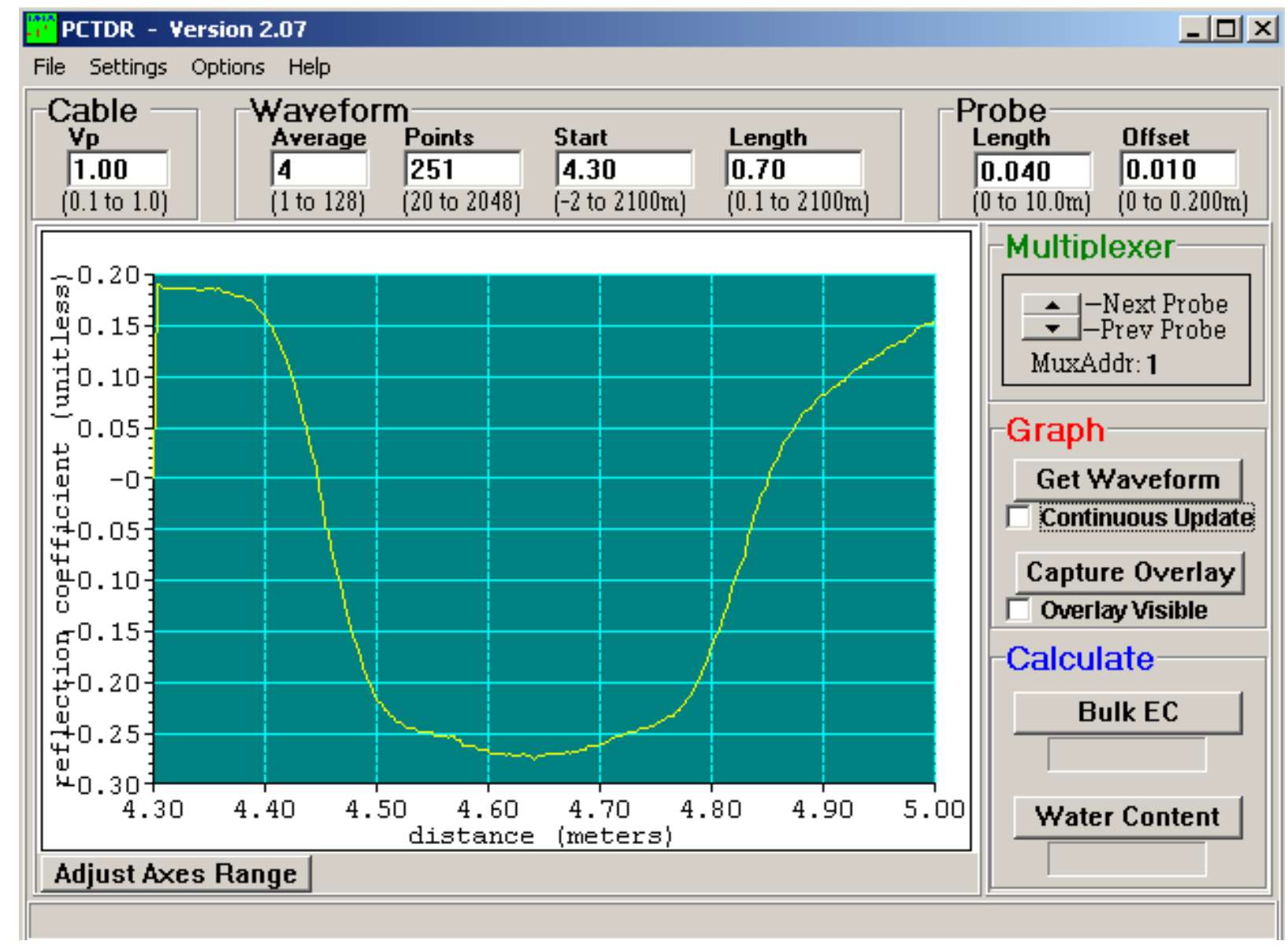

Fig. 3.2 Illustration of PC-TDR toolbox to find cable and window lengths

\subsection{Heat Pulse}

In 2003, Ren et al. (2003) applied heat pulse method to determine thermal properties of the soil as well as soil volumetric water content. Field studies by Bristow et al. (1993) showed that applying this method is beneficial since the costs and the disturbance of the soil are minimal. Therefore, in this study the heat pulse method was applied to determine soil thermal properties and 
soil water content for preliminary and main soil assessments. In 1996, a DOS base computer code so-called HPC (heat pulse C language) was developed by Welch et al. (1996) at Kansas State University. HPC program enables users to determine soil thermal diffusivity, volumetric heat capacity and thermal conductivity by fitting the transient temperature response to a heat pulse generated from a finite line heat source. The program models the pulse emitter as a finite line heat source. After tabular data of time versus temperature change are collected by the data-logger, they can be read by HPC program as a text file. There are certain values which need to be entered in HPC program. These values are: 1- the distance between the stainless-steel probe needles, 2duration of heat pulse generated by the central heater needle, 3- emitter half-length which is basically the half length of the probe needles embedded into the castable epoxy (probe handle) and 4- power generated per unit length of the emitter. For the in-house made T-TDR probes, correspondingly, these values are: $1-0.006 \mathrm{~m}, 2-7 \mathrm{~s}, 3-0.005 \mathrm{~m}$ and 4- $42.7 \mathrm{~W} / \mathrm{m}$.

\subsubsection{Theory}

Determination of soil volumetric heat capacity $(C)$ using heat pulse technique depends on the theory of heat conduction of short interval heat pulse. Fig.3.3 illustrates a sample graph of temperature rise variations vs. time. In an infinite medium, the change of temperature $\Delta T$ (temperature rise in ${ }^{\circ} \mathrm{C}$ ) as a function of time $t$ at a distance $d$ from the heat pulse source can be determined by (de Vries 1952 and Kluitenberg et al. 1993):

$$
\Delta T(d, t)=\frac{q}{4 \pi D_{a} C}\left[E_{i}\left(\frac{-d^{2}}{4 D_{a}\left(t-t_{o}\right)}\right)-E_{i}\left(\frac{-d^{2}}{4 D_{a} t}\right)\right] \quad \text { for } t>t_{o}
$$

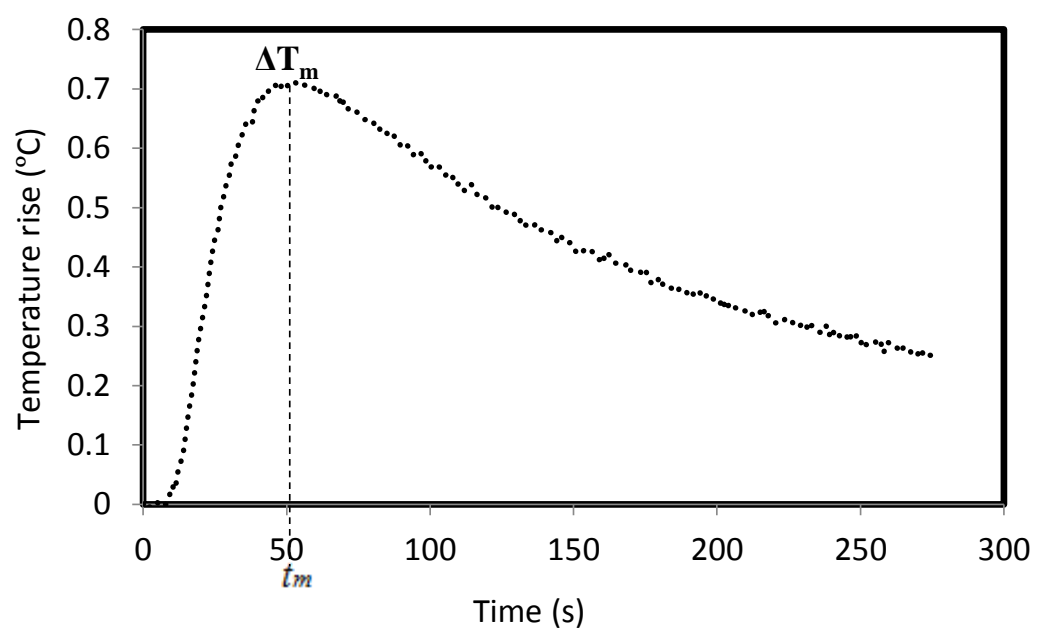

Fig. 3.3 Sample graph of temperature rise variations vs. time 
where $t$ is the time $(\mathrm{s}), t_{o}$ is the heat pulse duration $(\mathrm{s}), q$ is the heating power per unit length of the emitter $(\mathrm{W} / \mathrm{m}), d$ is the radial distance from the emitter $(\mathrm{m}), D_{a}$ is the thermal diffusivity of the soil $\left(\mathrm{m}^{2} / \mathrm{s}\right), C$ is the volumetric heat capacity of the soil $\left(\mathrm{J} / \mathrm{m}^{3} \cdot \mathrm{K}\right)$ and $E_{i}(x)$ is the exponential integral function (Abramowitz and Stegun 1972). Through the nonlinear regression method (Bristow et al. 1995 and Welch et al. 1996) to fit the temperature response data to Eq. (3.5), the thermal diffusivity and volumetric heat capacity of the soil can be determined. The expression for thermal diffusivity can be derived by differentiating Eq. (3.5) with respect to time (Kluitenberg 1995):

$D_{a}=\frac{d^{2}}{4}\left[\frac{\frac{1}{\left(t_{m}-t_{0}\right)}-\frac{1}{t_{m}}}{\ln \left(\frac{t_{m}}{t_{m}-t_{0}}\right)}\right]$

where $t_{m}$ indicates the time in which temperature reach maximum $\Delta \mathrm{T}_{\mathrm{m}}$. By rearranging Eq. (3.5) and replacing $t$ by $t_{m}$ and $\Delta \mathrm{T}$ by $\Delta \mathrm{T}_{\mathrm{m}}$, i.e. maximum temperature difference, volumetric heat capacity can be determined as

$C=\frac{q}{4 \pi D_{a} \Delta T_{m}}\left[E_{i}\left(\frac{-d^{2}}{4 D_{a}\left(t_{m}-t_{o}\right)}\right)-E_{i}\left(\frac{-d^{2}}{4 D_{a} t_{m}}\right)\right]$

The thermal conductivity can be computed using the following relation:

$k=C D_{a}$

The volumetric heat capacity can also be calculated from the following equation (Campbell et al. 1991):

$C=\rho_{b} c_{s}+\rho_{w} c_{w} \theta$

By rearranging Eq. (3.9), volumetric water content can be obtained as

$\theta=\left(C-\rho_{b} c_{s}\right) / \rho_{w} c_{w}$

\subsection{Construction Procedure of T-TDR Probe}

The construction of T-TDR probe was a delicate and time-consuming procedure. There are potential challenges associated with the process of construction and assembly. These challenges 
include, but not limited to, short circuiting, weak spots and breakage of the probe which may occur throughout the construction process. In this study, although it was tried to present detailed procedure, successful construction of a T-TDR probe requires developed skills by hands-on experience. Fig. 3.4 shows the image of a completed T-TDR probe with the labels of the main components. Each T-TDR probe has one stainless steel heater needle, positioned in the middle of the probe's head, and two stainless steel thermocouple needles, positioned on the sides of the probe's head. Appendix A.1 presents a list of materials used for the construction of T-TDR probes.

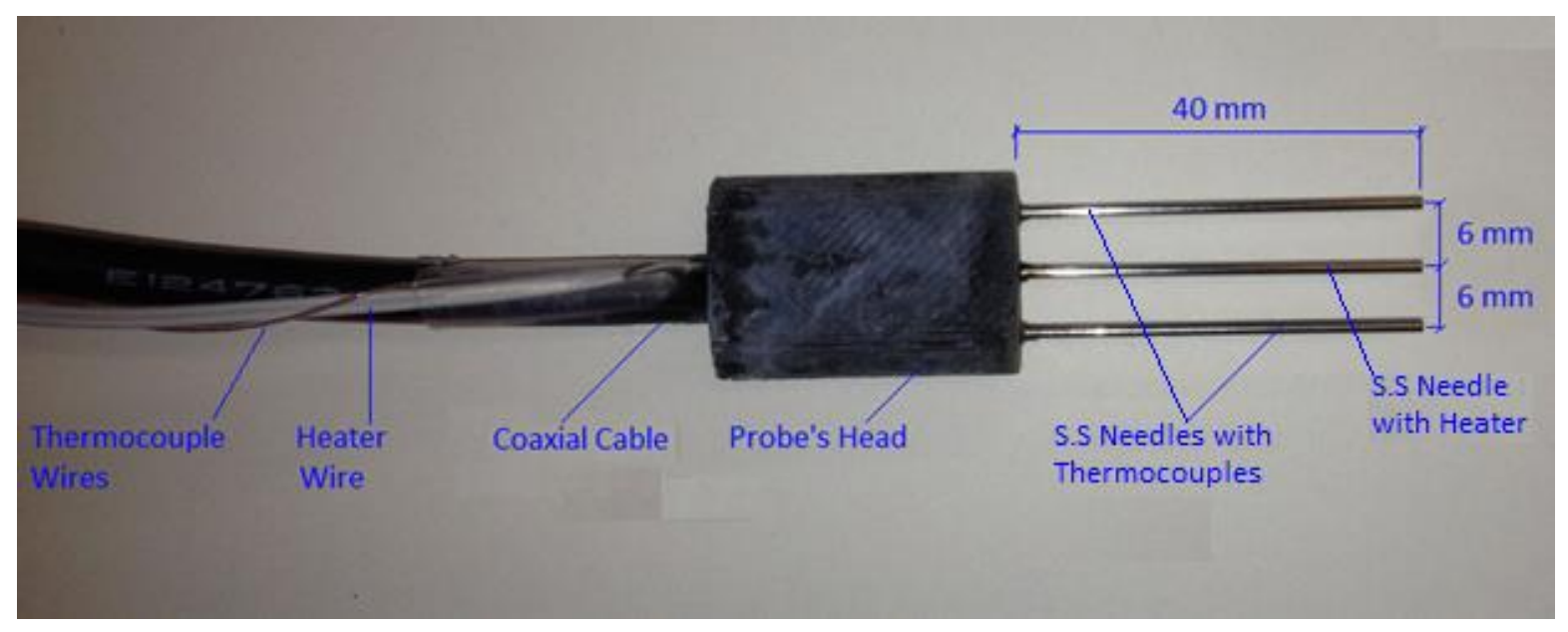

Fig. 3.4 An in-house made T-TDR Probe

\subsubsection{Heater Needle}

The heater needle is a 50-mm long 18 gauge stainless steel 304 tubing with outer and inner diameters of respectively 1.27 and $0.84 \mathrm{~mm}$. The resistance heater was selected to be 38 gauge Nichrome 80 wire with enamel coating. The electrical resistance of the heating wire is $138.7 \Omega / \mathrm{m}$. Following are the steps in making a heater needle:

1- Secure the needle to a wooden plate using a glue tape as shown in Fig. 3.5.

2- Cut $255 \mathrm{~mm}$ of the heater wire off from the spool, insert it into one needle until about 23.5 $\mathrm{mm}$ of one end is left out of the needle and tape it to the plate so that it is fixed.

3- Insert the free end of the heater wire through the needle until tip of the ' $U$ ' shaped wires was made just at the end of the needle. Again, insert back into the needle until ' $U$ ' shaped 
wires was made on the opposite side. For the third time, continue passing the wire through the needle so that a needle with 2 tag ends, each about $25 \mathrm{~mm}$ long, left out from the same end of the needle with all the "U" shaped wires placed just inside the needle.

Care should be taken to verify that there are no wires extended from the needle's ends other than the tag ends which are going to be connected to the electrical power.

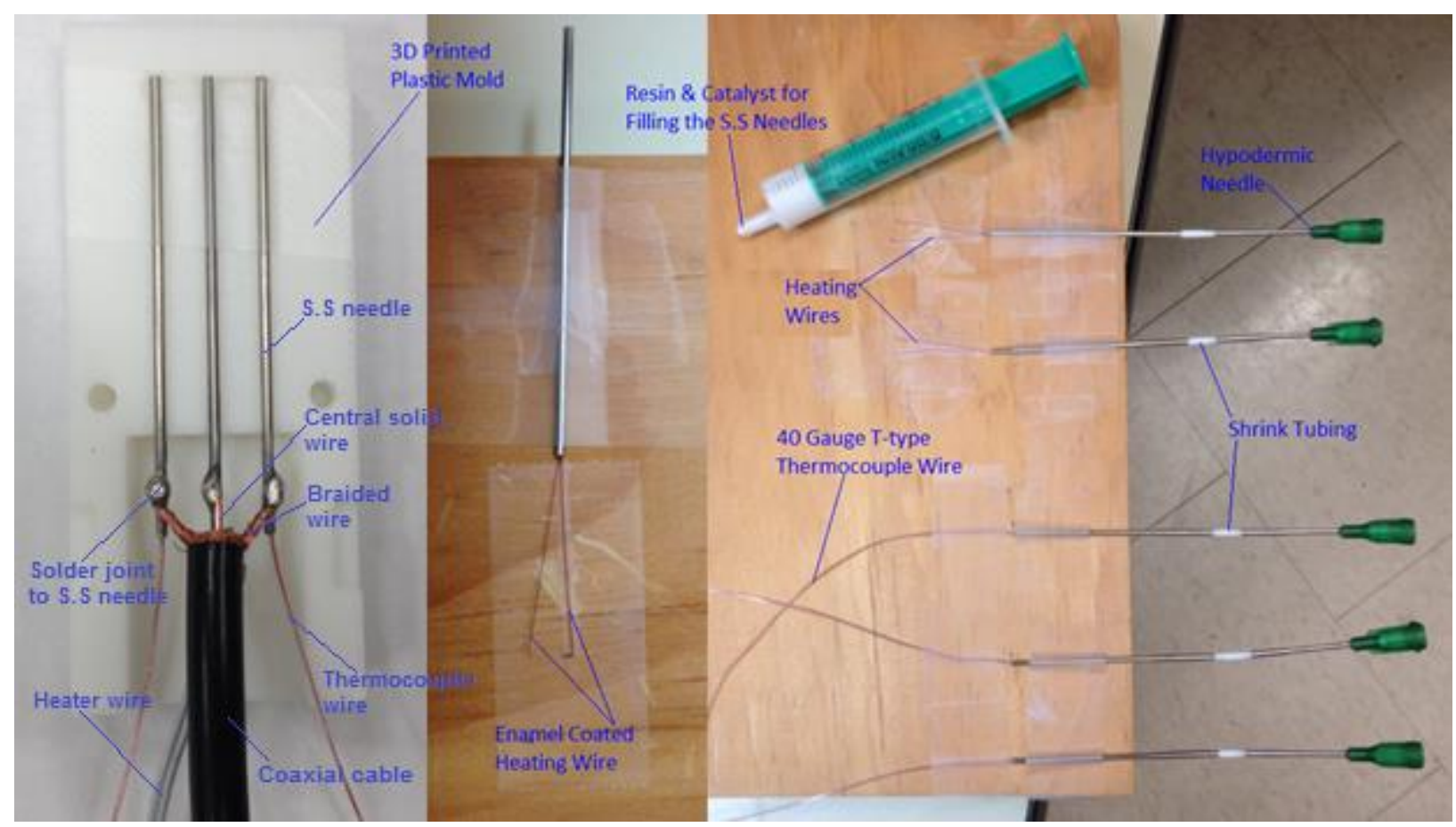

Fig. 3.5 The photo shoots of uncompleted T-TDR probe during the process of construction

\subsubsection{Thermocouple Needle}

The thermocouple wires used in this study was type-T 40 AWG OMEGA copper-constantan wires with duplex insulated layers. Although it is quite challenging to work with such fine thermocouple wires, it would deliver fast-response and high-precision measurement of temperature. In this study the experimental tests were transient, i.e. time dependent, therefore to achieve high level of accuracy it was important to measure temperature variations in very short period of time. Following are the steps in making a thermocouple needle:

1- Secure the needle to a wooden plate using a glue tape. 
2- Cut about $2 \mathrm{~m}$ of the type-T 40 AWG OMEGA thermocouple wires off from the spool and peel off $40 \mathrm{~mm}$ of outer insulated layer at both ends.

3- Mark $31 \mathrm{~mm}$ of copper (blue insulation) and constantan (red insulation) from the end which will be soldered.

4- Peel of $5 \mathrm{~mm}$ of both copper wire and constantan wire from both ends of the thermocouple wires and twist copper and constantan together only on one end.

5- Dip the end of the twisted wires into solder flux and solder the fluxed tip to make a small thermocouple junction, i.e. about $0.25 \mathrm{~mm}$ long.

6- Thread the junction through the needle until the point marked in step 3 and tape both thermocouple wires and the needle to the wooden plate.

7- Place both positive and negative guide ends of the ohmmeter on the peeled off copperconstantan wires on the opposite end of twisted wires made on step 4 to check whether the thermocouple junction is good.

Note that in steps 4 and 5 high level of skills are required and may need to work with a magnifier.

\subsubsection{Needle Filling}

After the resistance heater wire and the thermocouple wires were positioned in the needles they must be filled with specific type of epoxy. In this study Omega-bond 101 Resin and Catalyst was used for filling the needles. The process of filling was executed using 18 AWG blunt hypodermic needle with associated syringe. The hypodermic needle can be connected to stainless steel needles via heat-shrink tubing as shown in Fig. 3.5. Following are the steps in filling a needle:

1- Cut about $25 \mathrm{~mm}$ of heat-shrink tubing and insert hypodermic needle in for about $10 \mathrm{~mm}$. Do the same this time for needle to be filled with epoxy so that needles are connected. A heat gun can be used to seal heat-shrink tubing on the needles.

2- Mix equal quantities of Omega-bond 101 Resin and Catalyst and stir well. Then, load a syringe with the mixture. 
3- Insert the tip of syringe into hypodermic needle and squeeze out the mixture until it was seen that it is just extruded from the other end of the stainless steel needle. Wipe away the extruded mixture at the needle's end carefully.

4- Let the mixture to cure for about 15 hours. Then, remove the heat-shrink tubing by bending the heat-shrink tubing.

\subsubsection{Soldering Procedure}

The method of soldering copper and constantan wires to make a thermocouple junction was briefly described earlier. The trickiest part of soldering was, however, soldering coaxial cable to stainless steel needles. The coaxial cable includes a central solid wire and a layer of braided wires which are separated with the inner plastic shield. In this study the braided wires were divided in two equal parts and each part was twisted together. Also, the inner plastic shield over central wire was removed by $5 \mathrm{~mm}$ to expose the wire. Both outer and inner shields of the coaxial cable were removed using stripper with circular motion. The left side of Fig. 3.5 illustrates how coaxial cable wires were soldered on the needles. Following are the steps to solder coaxial cable wires onto stainless steel needles:

1- Place heater needle in the center and the other two thermocouple needles at the sides in Polyamid plastic mold and secure them carefully using glue tape.

2- Cover the entire needles with the tape except for the first $10 \mathrm{~mm}$ end of the needles which will be soldered.

3- Use rough abrasive sandpaper and gently remove the oxide layer on the exposed side of the needles. The indication of successful removal of the oxide layer is when the shininess of stainless steel is disappeared.

4- Carefully clean the surfaces and place the solder flux ${ }^{8}$ at desire points.

5- Hold the coaxial cable with a support stand and carefully adjust the central wire tip and twisted wires tips at the points where the solder flux was placed on the needles.

6- Use a soldered gun to heat up the central needle in the middle where will be soldered to the coaxial central wire.

${ }^{8}$ Qatey No. 5 NSF-61 
7- Carefully hold the central wire tip on the needle where solder flux was on top and drop small portion of solder $^{9}$ at the interface of needle and coaxial central wire.

8- Repeat step 6 and 7 to solder the other two twisted wires on the thermocouple needles.

For the case of heater needle following are the steps:

1- Carefully remove about $15 \mathrm{~mm}$ of the enamel coating from the end of resistance heater wire to expose the bare wire using very five abrasive sandpaper.

2- Clean the bare wire and carefully cut $10 \mathrm{~mm}$ of it in such a way that at least $5 \mathrm{~mm}$ bare wire left to be soldered to a 2-m long 28 AWG lead wire.

3- Drop the solder flux on each bare wire and carefully solder the two ends of heater wire to the lead wires.

4- Delicately soak the soldered parts into a liquid sealant ${ }^{10}$ and let it dry for at least one hour.

5- Use the ohmmeter to see if any short circuiting exist. This can be done by holding one of the ohmmeter's guides on one end of heater wire and the other on stainless steel needle. This should be done for both ends of the heater wire.

\subsubsection{Probe Casting}

Appendix B.1 presents engineering drawings of Polyamid plastic mold used for the probe's head casting in this study. United Resin El-Cast with 641 hardener was used for the probe casting. United Resin El-Cast was selected because it can be cured at the room temperature without any further treatment and can withstand temperatures up to $120^{\circ} \mathrm{C}$. The ratio of resin-hardener mixture is 5 to 1 . Following are the steps of probe casting in this study.

1- Ensure that all the needle ends are rested on the mold properly. Check for any bare wire touching each other and let the tapes to stay on the needles.

2- Carefully adjust the support stand, holding coaxial cable, in such a way that the mold and the needles become suspended vertically as shown in Fig. C.5 in Appendix C.

3- Spray lubricant release agent on the entire mold. Also, spray on the other half of the mold.

\footnotetext{
${ }^{9}$ Bernzomatic, Silver Bearing, lead free 1.6-mm SRC300

${ }^{10}$ Lepage, Gel epoxy
} 
4- Use another support stand to hold two clamps. Then clamp the two half molds together firmly making sure that the exerted pressure is uniformly spread on the molds.

5- Adjust the two support stands so that the cavity between the two half molds is suspended vertically without any slope.

6- Tape the two thermocouples and the heater wire together with coaxial cable at the section just out of the mold cavity.

7- Adjust the two support stands so that coaxial cable and the attached wires are in the center of the cavity.

8- Mix resin and hardener in clean $50 \mathrm{ml}$ beaker with the ratio of 5 to 1 and stir well.

9- Pour the casting mixture very slowly into the cavity until it is completely filled up. Note: make sure no air bubbles are generated during filling the mold cavity.

10- Let the cast to be cured for at least 72 hours before de-casting the mold.

\subsection{Digital Control System}

In this study, the operation of T-TDR probes was controlled by Campbell Scientific digital control system. The operation of the probes is composed of measuring temperatures, generating heat pulses, measuring electrical powers supplied to the heaters, sending electromagnetic signals and receiving the reflected waveforms. Digital control system is composed of two main parts. These parts are data acquisition (DAQ) system and software. In fact DAQ is a bridge between apparatus and the software. The software can be compared to a brain component of a system as a whole. The software used in this study was Campbell Scientific Logger-Net which was compatible with the DAQ. Logger-Net is composed of CRBasic editor and data monitoring toolbox. DAQ was composed of data logger (CR1000), thermocouple multiplexer (AM25T), TDR system (TDR100) and power relay controller (SDMCD-16S). Fig. 3.6 illustrates a flow chart of experimental setup algorithm. TDR system was introduced earlier in this chapter. The following subsections are brief introductions to the components of Campbell Scientific digital control system which were employed in this experimental study and are intended only for general information. In Appendix C, Fig. C.6 illustrates Campbell Scientific digital system including relay controller (SDMSCD16S), data logger (CR-1000), TDR system (TDR-100) and thermocouple multiplexer (AM25T). 


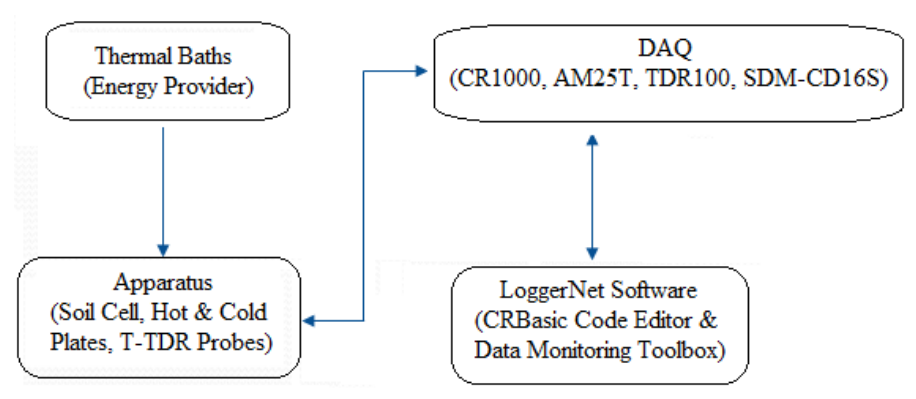

Fig. 3.6 Flow chart of experimental setup algorithm

\subsubsection{Programming CRBasic}

The CRBasic code editor is a programming tool in Logger-Net which can be used with Campbell Scientific data loggers which is CR1000 in this study. Generally CRBasic code editor is a tool which is intended for the programmers who need to have specific and unique control over the data logger. The structure of the program flow, logic and syntax of CRBasic program code is comparable with BASIC language programming. For the present experiment, a comprehensive CRBasic code was developed to perform various tasks during an experimental run, such as control timing, simultaneous measurements of temperatures, simultaneous operation of TDR probes, simultaneous control of heat pulses, simultaneous measurements of differential voltages across shunt resistances, and storage of all measured raw data in memory. Once an experimental run is completed, the experimentalist can save the raw data into an Excel file for post-processing and date reduction.

\subsubsection{Data Logger CR1000}

CR1000 is one of the components of data acquisition system. It can be power by $12 \mathrm{~V}$-DC source which was PS100 in this study. CR1000 executes CRBasic program instructions which include collecting data over time and controlling other DAQ components according to the instructions of the program. CR1000 is a widely-used data logger and it is compatible with various types of sensors.

\subsubsection{Power Relay Controller SDM-CD16S}

SDM-CD16S was used to control the required power on each T-TDR probes to generate the heat pulse. The program instructions were being executed by SDM-CD16S via data logger. 
This relay controller can provide up to 16 DC voltage outputs, however only 5 DC voltage outputs were utilized for all 5 probes. Although the relay could be powered by PS100, an external DC power supply (shown in Appendix C Fig. C.7) was used to power up the relay controller since simultaneous heat pulse generation for the probes required high voltage potential which was out of PS100 power aptitude. The probes resistance heaters were connected to relays positive and negative channels. The positive channels were connected to the heaters via 1- $\Omega$ shunt resistance in series so that the current (A) could be computed by differential voltage measurement across each shunt resistance. Once the current is determined, the electrical power supplied to the heater needle can be calculated as

$P=I^{2} R$

where $I$ is the electrical current (A) supplied to the heater, and $R$ is the electrical resistance of the heating wire in the heater needle. At higher temperature, the resistance increases according to the following relation.

$R(T)=R_{o}\left[1+\varphi\left(T-T_{o}\right)\right]$

where $R_{o}$ is the reference resistance at $T_{o}\left(R_{o}=27.47 \Omega\right.$ at $T_{o}=20^{\circ} \mathrm{C}$ for the heater needle), and $\varphi$ is the temperature coefficient of the heating wire. For the Nichrome 80 wire, $\varphi=1.096 \times 10^{-4}$ per degree Celsius.

\subsubsection{Thermocouple Multiplexer AM25T}

There were a total of twelve thermocouples used in this study for every experimental test. The benefit of using AM25T is its capability of providing up to 25 thermocouple terminals which are sequentially connected to a common differential channel. The AM25T has a built-in temperature reference junction for the thermocouples. The output from AM25T differential channel is connected to a differential input channel on the CR1000 for measuring the differential voltages generated by the thermocouples, and hence calculating the measured temperatures of the thermocouples. 


\section{CHAPTER 4}

\section{EXPERIMENTAL UNCERTAINTY ANALYSIS}

It is common to expect certain level of uncertainty in an experimental study. Therefore, in order to acquire reliable confidence level of experimental results, main sources of uncertainty must be identified and analyzed. Generally these sources of uncertainties are associated with the equipment specifications, system parameters, configuration and computational process. In this chapter, an experimental uncertainty analysis was carried out to estimate the uncertainties of the experimentally-determined quantities. In this study root-sum-square (RSS) technique was taken to obtain overall uncertainty according to the classic work of Kline and McClintock (1953).

\subsection{Uncertainty Analysis of Heat Pulse Technique}

Heat pulse technique was established to allow for concurrent measurement of soil thermal properties such as volumetric heat capacity, thermal diffusivity and thermal conductivity. The method of heat pulse is based on the theory for heat conduction through the soil away from the line heat source for a short time. In this experimental study, potential source of uncertainty was studied based on the method used by Kluitenberg et al. (1995) which is also recommended for various types of soils. The accuracy of the heat pulse method in measuring thermal properties can be evaluated by computing and systematically investigating potential sources of uncertainty. These potential sources of uncertainty include uncertainty estimations in measurements of required inputs such as delivered heat pulse, duration of delivered heat pulse, heat pulse maximum temperature, spacing between the probes and the time it takes to reach the heat pulse maximum temperature.

\subsubsection{Theory}

Recalling from Chapter 3, the expression for thermal diffusivity has been presented by Kluitenberg et al. (1995).

$D_{a}=\frac{d^{2}}{4}\left[\frac{\frac{1}{\left(t_{m}-t_{0}\right)}-\frac{1}{t_{m}}}{\ln \left(\frac{t_{m}}{t_{m}-t_{0}}\right)}\right]$

where $t_{m}$ indicates the time in which temperature reach maximum. The volumetric heat capacity can be expressed as 
$C=\frac{q}{4 \pi D_{a} \Delta T_{m}}\left[E_{i}\left(\frac{-d^{2}}{4 D_{a}\left(t_{m}-t_{o}\right)}\right)-E_{i}\left(\frac{-d^{2}}{4 D_{a} t_{m}}\right)\right]$

The thermal conductivity can be obtained by the product of Eqs. (4.1) and (4.2). In this study, it was assumed that the soil is homogenous and isotropic with no contact resistance ${ }^{11}$ between the probe's stainless-steel needles and soil. It was also assumed that the thermocouples embedded in the probe's needles have precise temperature measurements. This indicates that the junction in the thermocouple has considerably large thermal conductivity and considerably small heat capacity.

\subsubsection{Measurement Uncertainty of Thermal Diffusivity}

Considering Eq. (4.1), it can be presumed that the uncertainty in determination of thermal diffusivity $D_{a}$ is caused by measurement uncertainties in the probe spacing $d$, time for needle's temperature to reach maximum $t_{m}$ and duration of heat pulse $t_{0}$. Hence, the propagation uncertainty due to data reduction of Eq. (4.1) can be assessed using the root-sum-square (RSS) method, as follows:

$$
\delta D_{a}=\left[\left(\frac{\partial D_{a}}{\partial d} \delta d\right)^{2}+\left(\frac{\partial D_{a}}{\partial t_{m}} \delta t_{m}\right)^{2}+\left(\frac{\partial D_{a}}{\partial t_{0}} \delta t_{0}\right)^{2}\right]^{1 / 2}
$$

By solving partial derivatives and writing Eq. (4.3) as the first order approximation, relative uncertainty of thermal diffusivity is described as

$$
\frac{\delta D_{a}}{D_{a}} \approx\left[\left(2 \frac{\delta d}{d}\right)^{2}+\left(\varepsilon \frac{\delta t_{m}}{t_{m}}\right)^{2}+\left(-(1+\varepsilon) \frac{\delta t_{0}}{t_{0}}\right)^{2}\right]^{1 / 2}
$$

where

$$
\varepsilon=\frac{t_{m}}{\left(d^{2} / 4 D_{a}\right)}-\frac{t_{m}}{\left(t_{m}-t_{0}\right)}-1
$$

\subsubsection{Measurement Uncertainty of Volumetric Heat Capacity}

\footnotetext{
${ }^{11}$ Refers to discrete contact spots in which gaps with relatively lower thermal conductivity can be formed in between these spots so that most of the heat is forced to pass through only these contact spots.
} 
Considering Eq. (4.2), it can be presumed that the uncertainty in determination of volumetric heat capacity $C$ is caused by measurement uncertainties in energy per unit length of the emitter $q, \Delta T_{m}, d, t_{m}$ and $t_{0}$. Since Eq. (4.2) includes $D_{a}$, uncertainty in $C$ can also be affected by the uncertainty in $D_{a}$ as a consequence of measurement uncertainties in $d, t_{m}$ and $t_{0}$. Hence, the propagation uncertainty due to data reduction of Eq. (4.2) can be assessed using the RSS method, as follows:

$\delta C=\left[\left(\frac{\partial C}{\partial q} \delta q\right)^{2}+\left(\frac{\partial C}{\partial T_{m}} \delta T_{m}\right)^{2}+\left(\frac{\partial C}{\partial d} \delta d\right)^{2}+\left(\frac{\partial C}{\partial t_{m}} \delta t_{m}\right)^{2}+\left(\frac{\partial C}{\partial t_{0}} \delta t_{0}\right)^{2}+\left(\frac{\partial C}{\partial D_{a}} \frac{\partial D_{a}}{\partial d} \delta d\right)^{2}+\right.$

$\left.\left(\frac{\partial C}{\partial D_{a}} \frac{\partial D_{a}}{\partial t_{m}} \delta t_{m}\right)^{2}+\left(\frac{\partial C}{\partial D_{a}} \frac{\partial D_{a}}{\partial t_{0}} \delta t_{0}\right)^{2}\right]^{1 / 2}$

By solving partial derivatives and writing Eq. (4.5) as the first order approximation, relative uncertainty of volumetric heat capacity is described as

$$
\frac{\delta C}{C} \approx\left[\left(\frac{\delta q}{q}\right)^{2}+\left(-\frac{\delta T_{m}}{T_{m}}\right)^{2}+\left(-2 \frac{\delta d}{d}\right)^{2}+\left(\sigma \frac{\delta t_{m}}{t_{m}}\right)^{2}+\left((1-\sigma) \frac{\delta t_{0}}{t_{0}}\right)^{2}\right]^{1 / 2}
$$

where

$\sigma=-\varepsilon(1+\xi)$

and

$\xi=\frac{\exp \left[-\frac{d^{2}}{4 D_{a}\left(t_{m}-t_{0}\right)}\right]-\exp \left(-\frac{d^{2}}{4 D_{a} t_{m}}\right)}{E i\left[-\frac{d^{2}}{4 D_{a}\left(t_{m}-t_{0}\right)}\right]-E i\left(-\frac{d^{2}}{4 D_{a} t_{m}}\right)} \quad$ and $\quad \varepsilon=\frac{t_{m}}{\left(d^{2} / 4 D_{a}\right)}-\frac{t_{m}}{\left(t_{m}-t_{0}\right)}-1$

\subsubsection{Measurement Uncertainty of Thermal Conductivity}

Recalling from Chapter 3, Eq. (3.8) represents thermal conductivity $(k)$ as a product of $C$ and $D_{a}$. Thus, the propagation uncertainty due to data reduction of Eq. (3.8) can be assessed using the RSS method, as follows:

$$
\delta k=\left[\left(\frac{\partial k}{\partial C} \delta C\right)^{2}+\left(\frac{\partial k}{\partial D_{a}} \delta D_{a}\right)^{2}\right]^{1 / 2}
$$


By solving partial derivatives and writing Eq. (4.7) as the first order approximation, the relative uncertainty of thermal conductivity is described as

$$
\frac{\delta k}{k}=\left[\left(\frac{\delta C}{C}\right)^{2}+\left(\frac{\delta D_{a}}{D_{a}}\right)^{2}\right]^{1 / 2}
$$

Substituting the first two terms in Eq. (4.8) by Eqs. (4.4) and (4.6), the relative uncertainty of thermal conductivity can be rearranged as

$$
\frac{\delta k}{k} \approx\left[\left(\frac{\delta q}{q}\right)^{2}+\left(-\frac{\delta T_{m}}{T_{m}}\right)^{2}+\left((\varepsilon+\sigma) \frac{\delta t_{m}}{t_{m}}\right)^{2}+\left(-(\varepsilon+\sigma) \frac{\delta t_{0}}{t_{0}}\right)^{2}\right]^{1 / 2}
$$

\subsubsection{Measurement Uncertainty of Volumetric Water Content}

The volumetric heat capacity of a soil can be calculated from the following equation (Campbell et al. 1991):

$C=\rho_{b} c_{s}+\rho_{a} c_{a}(\eta-\theta)+\rho_{w} c_{w} \theta$

By rearranging Eq. (4.10), the volumetric water content can be determined as

$\theta=\frac{C-\rho_{b} c_{s}-\rho_{a} c_{a} \eta}{\rho_{w} c_{w}-\rho_{a} c_{a}}$

Since $\rho_{a} c_{a} \ll \rho_{w} c_{w}$ and $\rho_{a} c_{a} \ll \rho_{b} c_{s}$, Eq. (4.11) can be simplified to

$\theta=\frac{c-\rho_{b} c_{s}}{\rho_{w} c_{w}}$

In Eq. (4.12), the quantity $\rho_{b} c_{s}$ is the volumetric heat capacity of dry soil, and it can be determined using the heat pulse method beforehand.

Applying the root-sum-square (RSS) method, the uncertainty can be determined from the following relation:

$\delta \theta=\left[\left(\frac{\partial \theta}{\partial C} \delta C\right)^{2}+\left(\frac{\partial \theta}{\partial\left(\rho_{b} c_{s}\right)} \delta\left(\rho_{b} c_{S}\right)\right)^{2}+\left(\frac{\partial \theta}{\partial \rho_{w}} \delta \rho_{w}\right)^{2}++\left(\frac{\partial \theta}{\partial c_{w}} \delta c_{w}\right)^{2}\right]^{1 / 2}$ 
Hence, the relative uncertainty becomes

$\frac{\delta \theta}{\theta}=\left[\left(\frac{\delta C}{C-\rho_{b} c_{s}}\right)^{2}+\left(-\frac{\delta\left(\rho_{b} c_{s}\right)}{C-\rho_{b} c_{s}}\right)^{2}+\left(-\frac{\delta \rho_{w}}{\rho_{w}}\right)^{2}+\left(-\frac{\delta c_{w}}{c_{w}}\right)^{2}\right]^{1 / 2}$

\subsubsection{Summary of the Uncertainty Calculations for Soil Thermal Properties and Volumetric Water Content}

In this study the computed uncertainties, associated with the heat pulse technique in measuring the thermal properties and volumetric water content were implemented for the cases of dry, half saturated and saturated soil (Matilda) each at two different temperatures. The maximum uncertainty was found to be about $\pm 7.2 \%$ in the measurement of the thermal conductivity of the dry soil sample at $90^{\circ} \mathrm{C}$. The minimum uncertainty was found to be about $\pm 2 \%$ in the measurement of the volumetric heat capacity of saturated soil at $20^{\circ} \mathrm{C}$. Table 4.1 presents the typical parameters used in this study to calculate uncertainties in measurements of thermal properties and volumetric water contents. Table 4.2 presents the summary of the results of measurement uncertainties in the calculations of the soil thermal properties and volumetric water contents at different soil saturation degrees and temperatures.

Table 4.1 Typical input parameters used for uncertainty calculations

\begin{tabular}{|c|c|c|c|c|c|c|c|c|c|c|}
\hline & $\begin{array}{c}\delta T_{m} \\
\left({ }^{\circ} \mathrm{C}\right)\end{array}$ & $\begin{array}{c}T_{m} \\
\left({ }^{\circ} \mathrm{C}\right)\end{array}$ & $\begin{array}{c}\delta d \\
(\mathrm{~mm})\end{array}$ & $\begin{array}{c}d \\
(\mathrm{~mm})\end{array}$ & $\begin{array}{c}\delta t_{m} \\
(\mathrm{~s})\end{array}$ & $\begin{array}{c}t_{m} \\
(\mathrm{~s})\end{array}$ & $\begin{array}{c}\delta t_{0} \\
(\mathrm{~s})\end{array}$ & $\begin{array}{c}t_{0} \\
(\mathrm{~s})\end{array}$ & $\begin{array}{c}\delta q \\
(\mathrm{~W} / \mathrm{m})\end{array}$ & $\begin{array}{c}q \\
(\mathrm{~W} / \mathrm{m})\end{array}$ \\
\hline $\begin{array}{c}\text { Soil } \mathrm{S}_{\mathrm{r}}=0 \\
\text { at } 90^{\circ} \mathrm{C}\end{array}$ & \pm 0.5 & 90.29 & \pm 0.1 & 6 & \pm 0.75 & 53.5 & \pm 0.01 & 7 & \pm 0.16 & 42.69 \\
\hline $\begin{array}{c}\text { Soil } \mathrm{S} \mathrm{r}=0 \\
\text { at } 20^{\circ} \mathrm{C}\end{array}$ & \pm 0.5 & 20.93 & \pm 0.1 & 6 & \pm 0.75 & 57.2 & \pm 0.01 & 7 & \pm 0.16 & 42.74 \\
\hline $\begin{array}{c}\text { Soil } \mathrm{S}= \\
0.5 \text { at } 90^{\circ} \mathrm{C}\end{array}$ & \pm 0.5 & 90.65 & \pm 0.1 & 6 & \pm 0.75 & 15.3 & \pm 0.01 & 7 & \pm 0.16 & 42.67 \\
\hline $\begin{array}{c}\text { Soil } \mathrm{S}_{\mathrm{r}}= \\
0.5 \text { at } 20^{\circ} \mathrm{C}\end{array}$ & \pm 0.5 & 20.22 & \pm 0.1 & 6 & \pm 0.75 & 16.8 & \pm 0.01 & 7 & \pm 0.16 & 42.79 \\
\hline $\begin{array}{c}\text { Soil } \mathrm{Sr}=1 \\
\text { at } 90^{\circ} \mathrm{C}\end{array}$ & \pm 0.5 & 90.71 & \pm 0.1 & 6 & \pm 0.75 & 11.2 & \pm 0.01 & 7 & \pm 0.16 & 42.81 \\
\hline $\begin{array}{c}\text { Soil } \mathrm{S}=1 \\
\text { at } 20^{\circ} \mathrm{C}\end{array}$ & \pm 0.5 & 20.69 & \pm 0.1 & 6 & \pm 0.75 & 11.5 & \pm 0.01 & 7 & \pm 0.16 & 42.83 \\
\hline
\end{tabular}


Table 4.2 Results of uncertainties in measurement of the thermal properties and water content

\begin{tabular}{|c|c|c|c|c|c|c|c|}
\hline & $\begin{array}{c}\mathrm{C} \\
\left(\mathrm{J} / \mathrm{m}^{3} \cdot \mathrm{K}\right)\end{array}$ & $\begin{array}{c}\mathrm{D}_{\mathrm{a}} \\
\left(\mathrm{m}^{2} / \mathrm{s}\right)\end{array}$ & $\begin{array}{c}\mathrm{k} \\
(\mathrm{W} / \mathrm{m} \cdot \mathrm{K})\end{array}$ & $\begin{array}{c}\delta \mathrm{C} / \mathrm{C} \\
(\%)\end{array}$ & $\begin{array}{c}\delta D_{a} / D_{a} \\
(\%)\end{array}$ & $\begin{array}{c}\delta \mathrm{k} / \mathrm{k} \\
(\%)\end{array}$ & $\begin{array}{c}\delta \theta / \theta \\
(\%)\end{array}$ \\
\hline $\begin{array}{c}\text { Soil } \mathrm{S}_{\mathrm{r}}=0 \\
\text { at } 90^{\circ} \mathrm{C}\end{array}$ & 68325 & $9.10 \times 10^{-6}$ & 0.61 & \pm 4.2 & \pm 5.8 & \pm 7.2 & - \\
\hline $\begin{array}{c}\text { Soil } \mathrm{S}_{\mathrm{r}}=0 \\
\text { at } 20^{\circ} \mathrm{C}\end{array}$ & 68325 & $3.80 \times 10^{-6}$ & 0.26 & \pm 3.6 & \pm 3.6 & \pm 5.2 & - \\
\hline $\begin{array}{c}\text { Soil } \mathrm{S}_{\mathrm{r}}= \\
0.5 \text { at } 90^{\circ} \mathrm{C}\end{array}$ & 2000563 & $7.02 \times 10^{-7}$ & 1.4 & \pm 3.1 & \pm 4.3 & \pm 5.1 & \pm 2.2 \\
\hline $\begin{array}{c}\text { Soil } \mathrm{S}_{\mathrm{r}}= \\
0.5 \text { at } 20^{\circ} \mathrm{C}\end{array}$ & 1923783 & $6.77 \times 10^{-7}$ & 1.3 & \pm 2.1 & \pm 3.4 & \pm 4 & \pm 3.23 \\
\hline $\begin{array}{c}\text { Soil } \mathrm{S}_{\mathrm{r}}=1 \\
\text { at } 90^{\circ} \mathrm{C}\end{array}$ & 4861298 & $3.78 \times 10^{-7}$ & 1.8 & \pm 2.8 & \pm 4.2 & \pm 5.3 & \pm 2.8 \\
\hline $\begin{array}{c}\text { Soil } \mathrm{S}_{\mathrm{r}}=1 \\
\text { at } 20^{\circ} \mathrm{C}\end{array}$ & 3991543 & $3.75 \times 10^{-7}$ & 1.5 & \pm 2 & \pm 3.3 & \pm 3.9 & \pm 2 \\
\hline
\end{tabular}

\subsection{Uncertainty Analysis of Soil Sample Preparation}

The uncertainty of soil sample preparation based on degrees of saturation were estimated based on $95 \%$ confidence level. A number of 10 samples were prepared for every saturation degree and for each types of soil. The overall uncertainty for $95 \%$ confidence level can be defined as

$U_{0.95}=\left[B_{\bar{\theta}}^{2}+\left(t S_{\bar{\theta}}\right)^{2}\right]^{\frac{1}{2}}$

where $B_{\bar{\theta}}$ is the systematic uncertainty of the mean volumetric water content $\bar{\theta}$ due to specifications of tools and equipment used to prepare the samples, $S_{\bar{\theta}}$ is the standard deviation of the mean and $t$ is the student's $t$ multiplier for $95 \%$ confidence level and $n-1$ degrees of freedom. Standard deviation of the mean can be evaluated as:

$S_{\bar{\theta}}=\sqrt{\frac{\sum_{i=1}^{n}\left(\theta_{i}-\bar{\theta}\right)^{2}}{n(n-1)}}$

where $n$ is the number of samples and $\theta_{i}$ is the volumetric water content $\left(\mathrm{m}^{3} / \mathrm{m}^{3}\right)$ for each sample which is defined as

$\theta_{i}=S_{r} \eta_{i}$ 
where $\eta_{i}$ is porosity of soil for each sample and $S_{r}$ is a degree of saturation and can be defined as

$S_{r}=\frac{V_{w}}{V_{\text {void }}}$

In Eq. (4.18), $V_{w}$ and $V_{v o i d}$ are respectively volumes of water and void space in a soil sample. The porosity of soil can be calculated using the following equation:

$\eta=1-\frac{\rho_{b}}{\rho_{s}}$

where $\rho_{b}$ and $\rho_{s}$ are respectively bulk and solid densities of soil. From the process of sample preparation, the bulk density is the mass of dry soil per its volume $V_{\text {soil }}$. The volume of the soil $V_{\text {soil }}$ is the volume of the graduated beaker used in preparation of the soil samples and $V_{\text {soil }}=$ $200 m L \pm 2 m L$. Hence $S_{\mathrm{r}}$ can be rewritten as

$S_{r_{i}}=\frac{m_{w}}{\rho_{w} \eta_{i} V_{\text {soil }}}$

where

$\eta_{i}=1-\frac{\left(m_{\text {tot }}-m_{w}\right)}{\rho_{s} V_{\text {soil }}}$

where $m_{t o t}$ is the total mass of the wet soil, $m_{w}$ is the mass of water added to the dry soil, and $\rho_{w}$ is the density of water.

In Eq. (4.15) the systematic uncertainty can be expressed as

$B_{\bar{\theta}}=\frac{\delta \theta}{\theta} \bar{\theta}$

where $\theta$ is volumetric water content and from Eqs. (4.17) and (4.20). It can be expressed as

$\theta=\frac{m_{w}}{\rho_{w} V_{\text {soil }}}$

and $\frac{\delta \theta}{\theta}$ is relative uncertainty of water content. Applying the RSS method, the uncertainty can be determined from the following relation:

$\delta \theta=\left[\left(\frac{\partial \theta}{\partial m_{w}} \delta m_{w}\right)^{2}+\left(\frac{\partial \theta}{\partial \rho_{w}} \delta \rho_{w}\right)^{2}+\left(\frac{\partial \theta}{\partial V_{\text {soil }}} \delta V_{\text {soil }}\right)^{2}\right]^{1 / 2}$ 
Hence, the relative uncertainty becomes

$\frac{\delta \theta}{\theta}=\left[\left(\frac{\delta m_{w}}{m_{w}}\right)^{2}+\left(\frac{\delta \rho_{w}}{\rho_{w}}\right)^{2}+\left(\frac{\delta V_{\text {soil }}}{V_{\text {soil }}}\right)^{2}\right]^{1 / 2}$

\subsubsection{Summary of the Uncertainty Calculations for Soil Sample Preparation}

Tables 4.3 to 4.5 present the overall uncertainties in sample preparation at $95 \%$ confidence level which were determined in this study for three types of soils. The three types of soils are Matilda (ON-3), Ottawa sand (C-190) and Fort St. James or FSJ\#1 (BC-1). The details about the soils will be presented in Chapter 5. For each $S_{\mathrm{r}}$ and soil type, the overall uncertainty of the soil sample preparation was evaluated based on ten soil samples $(n=10)$ with the student's $t$ multiplier of 2.262 for $95 \%$ confidence and nine degrees of freedom. It can be seen that the maximum overall uncertainty in soil sample preparation is about $2.7 \%$ when $\mathrm{S}_{\mathrm{r}}$ is low.

Table 4.3 Overall uncertainty in sample preparation at 95\% confidence level for ON-3

\begin{tabular}{|c|c|c|c|c|c|c|c|c|c|}
\hline & $\begin{array}{c}\rho_{S} \\
\left(\mathrm{~kg} \cdot \mathrm{m}^{-3}\right)\end{array}$ & $\begin{array}{l}m_{w} \\
(\mathrm{~kg})\end{array}$ & $\begin{array}{c}m_{t o t} \\
(\mathrm{~kg})\end{array}$ & $\begin{array}{c}S_{\bar{\theta}} / \bar{\theta} \\
(\%)\end{array}$ & $\bar{\eta}$ & $\begin{array}{c}\bar{\theta} \\
\left(\mathrm{m}^{3} \cdot \mathrm{m}^{-3}\right)\end{array}$ & $\overline{S_{r}}$ & $\begin{array}{c}B_{\bar{\theta}} / \bar{\theta} \\
(\%)\end{array}$ & $\begin{array}{c}U_{0.95} / \bar{\theta} \\
(\%)\end{array}$ \\
\hline$S_{r}=0.25$ & 2706 & 0.023 & 0.315 & 0.387 & 0.46 & 0.116 & 0.252 & 1.48 & 1.72 \\
\hline$S_{r}=0.5$ & 2706 & 0.046 & 0.338 & 0.351 & 0.46 & 0.232 & 0.504 & 1.43 & 1.64 \\
\hline$S_{r}=1$ & 2706 & 0.092 & 0.384 & 0.225 & 0.46 & 0.463 & 1.01 & 1.42 & 1.51 \\
\hline
\end{tabular}

Table 4.4 Overall uncertainty in sample preparation at 95\% confidence level for C-190

\begin{tabular}{|c|c|c|c|c|c|c|c|c|c|}
\hline & $\begin{array}{c}\rho_{S} \\
\left(\mathrm{~kg} \cdot \mathrm{m}^{-3}\right)\end{array}$ & $\begin{array}{c}m_{w} \\
(\mathrm{~kg})\end{array}$ & $\begin{array}{c}m_{\text {tot }} \\
(\mathrm{kg})\end{array}$ & $\begin{array}{c}S_{\bar{\theta}} / \bar{\theta} \\
(\%)\end{array}$ & $\bar{\eta}$ & $\begin{array}{c}\bar{\theta} \\
\left(\mathrm{m}^{3} \cdot \mathrm{m}^{-3}\right)\end{array}$ & $\overline{S_{r}}$ & $\begin{array}{c}B_{\bar{\theta}} / \bar{\theta} \\
(\%)\end{array}$ & $\begin{array}{c}\boldsymbol{U}_{\mathbf{0 . 9 5}} / \overline{\boldsymbol{\theta}} \\
(\boldsymbol{\%})\end{array}$ \\
\hline$S_{r}=0.25$ & 2650 & 0.017 & 0.366 & 0.969 & 0.34 & 0.086 & 0.252 & 1.53 & $\mathbf{2 . 6 7}$ \\
\hline$S_{r}=0.5$ & 2650 & 0.034 & 0.384 & 0.599 & 0.34 & 0.172 & 0.505 & 1.44 & $\mathbf{1 . 9 8}$ \\
\hline$S_{r}=1$ & 2650 & 0.069 & 0.418 & 0.334 & 0.34 & 0.344 & 1.01 & 1.42 & $\mathbf{1 . 6 1}$ \\
\hline
\end{tabular}


Table 4.5 Overall uncertainty in sample preparation at 95\% confidence level for BC-1

\begin{tabular}{|c|c|c|c|c|c|c|c|c|c|}
\hline & $\begin{array}{c}\rho_{s} \\
\left(\mathrm{~kg} \cdot \mathrm{m}^{-3}\right)\end{array}$ & $\begin{array}{l}m_{w} \\
(\mathrm{~kg})\end{array}$ & $\begin{array}{c}m_{t o t} \\
(\mathrm{~kg})\end{array}$ & $\begin{array}{c}S_{\bar{\theta}} / \bar{\theta} \\
(\%)\end{array}$ & $\bar{\eta}$ & $\begin{array}{c}\bar{\theta} \\
\left(\mathrm{m}^{3} \cdot \mathrm{m}^{-3}\right)\end{array}$ & $\overline{S_{r}}$ & $\begin{array}{c}B_{\bar{\theta}} / \bar{\theta} \\
(\%)\end{array}$ & $\begin{array}{c}U_{0.95} / \bar{\theta} \\
(\%)\end{array}$ \\
\hline$S_{r}=0.25$ & 2650 & 0.025 & 0.294 & 0.851 & 0.51 & 0.128 & 0.250 & 1.47 & 2.42 \\
\hline$S_{r}=0.5$ & 2650 & 0.051 & 0.320 & 0.491 & 0.51 & 0.257 & 0.504 & 1.43 & 1.81 \\
\hline$S_{r}=1$ & 2650 & 0.102 & 0.370 & 0.403 & 0.51 & 0.511 & 1.00 & 1.42 & 1.69 \\
\hline
\end{tabular}

\subsection{Uncertainty Analysis of Temperature Measurement via T-TDR Probe}

One main possible source of uncertainty in this experiment was heat conduction via TTDR probes since thermal conductivity of stainless steel needles of the probe as well as thermocouple wire embedded in the probe's needles is significantly higher than surrounding soil. In fact, heat conduction through stainless steel needle and thermocouple wires could cause substantial uncertainty in temperature measurement. The purpose of this analysis is to estimate the temperature measurement uncertainty as a results of heat conduction through T-TDR probe. The methodology to analyze the uncertainty caused by the heat conduction through thermocouple wires was derived from Leong (1996).

\subsubsection{Problem Definition}

A thermocouple wire is embedded in stainless steel (S.S) hypodermic tube of T-TDR probe. It is assumed that the junction of the thermocouple has distance $L$ from the soil cell inner wall. Thermocouple wires as well as coaxial cable connected to the probe's handle are surrounded by an insulation layer with a total thickness of about $10 \mathrm{~cm}$ (4 inches) before they are extended infinitely long into the ambient air at temperature $T_{a}$, as depicted in Fig. 4.1. Table 4.6 tabulates the input data for uncertainty calculations due to conduction heat transfer via T-TDR probe. 


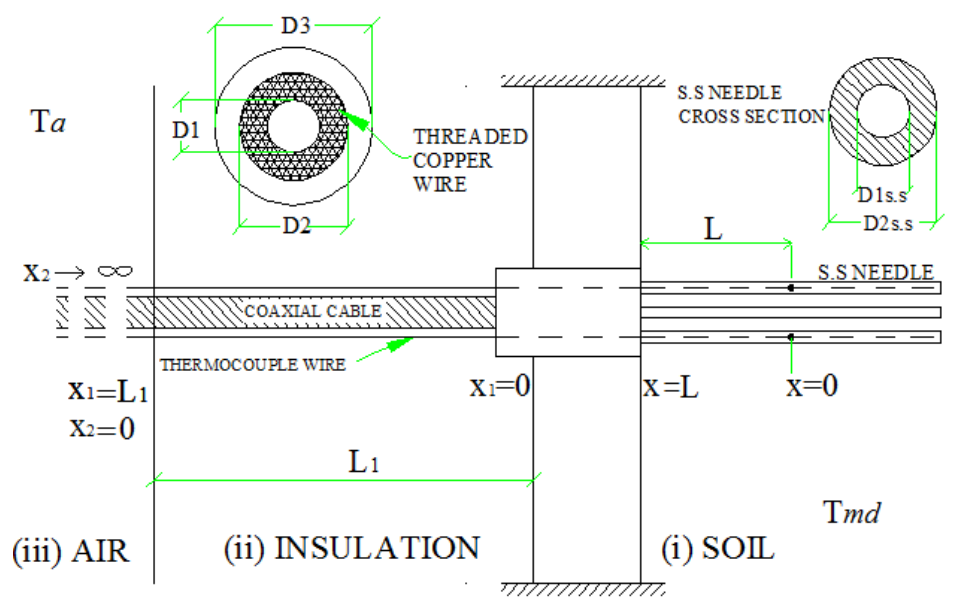

Fig. 4.1 Schematic of heat conduction problem as modelled: The probe's needles are inserted in soil, portion of coaxial cable is surrounded by insulation and extended infinitely into the air

\subsubsection{Uncertainty Caused by Heat Conduction from Thermocouple Wire}

Conduction heat loss over the entire length of thermocouple wire can be described by the following equations:

(i) Along thermocouple wire embedded in the T-TDR probe's S.S needle in the soil $(0 \leq$ $x \leq L)$ :

$\frac{\partial^{2} T}{\partial x^{2}}-\alpha_{m d}^{2}\left(T-T_{m d}\right)=0$

where

$\alpha_{m d}^{2}=\frac{h_{m d} P}{k_{\text {copper }} A}=\frac{2 h_{m d}}{k_{\text {copper }} a}$

and $h_{m d}=$ heat transfer coefficient between copper wire and soil medium.

(ii) Along thermocouple wire in insulation layer $\left(0 \leq x_{1} \leq L_{1}\right)$ :

$\frac{\partial^{2} T}{\partial x_{1}^{2}}-\alpha_{i n}^{2}\left(T-T_{a}\right)=0$ 
where

$\alpha_{\text {in }}^{2}=\frac{h_{\text {in }} P}{k_{\text {copper }} A}=\frac{2 h_{\text {in }}}{k_{\text {copper }} a}$

and $h_{\text {in }}=$ heat transfer coefficient between copper wire and outer surface of fiberglass insulation.

(iii) Along thermocouple wire in the ambient air $\left(0 \leq x_{2} \leq \infty\right)$ :

$\frac{\partial^{2} T}{\partial x_{2}^{2}}-\alpha_{a}^{2}\left(T-T_{a}\right)=0$

where

$\alpha_{a}^{2}=\frac{2 h_{a}}{k_{\text {copper }} a}$

and $h_{a}=$ heat transfer coefficient between copper wire and ambient air.

The heat from copper thermocouple wire to the soil flows through three thermal resistances which are connected in series. Fig. 4.2 illustrates three layers of thermal resistance from the thermocouple wire to the surface of S.S needle embedded in the soil. Considering Eq. (4.26), $h_{m d}$ is the heat transfer coefficient between the copper wire and soil and can be computed via the following equation:

$h_{m d}=1 /\left(\frac{\operatorname{aln} \frac{b}{a}}{k_{\text {teflon }}}+\frac{\operatorname{aln} \frac{c}{b}}{k_{\text {epoxy }}}+\frac{\operatorname{aln} \frac{d}{c}}{k_{s . s}}+\frac{a}{h_{c} d}\right)$

where $a=$ radius of copper wire, $b=$ outer radius of Teflon insulation layer, $c=$ outer radius of epoxy layer, $d=$ outer radius of S.S needle, $k_{\text {teflon }}=$ thermal conductivity of Teflon, $k_{\text {epoxy }}=$ thermal conductivity of epoxy, $k_{S . S}=$ thermal conductivity of stainless steel needle, and $h_{c}=$ thermal contact conductance at S.S needle-soil interface.

Considering Eq. (4.27), $h_{\text {in }}$ is the heat transfer coefficient between copper wire and outer surface of fiberglass insulation which can be determined by the following equation: 
$h_{\text {in }}=1 /\left(\frac{\operatorname{aln} \frac{b}{a}}{k_{\text {teflon }}}+\frac{\operatorname{aln} \frac{2 L_{1}}{b}}{k_{\text {in }}}\right)$

where $L_{1}=$ the thickness of fiberglass insulation covering the coaxial cable and the thermocouple wire, before the wire is exposed to the ambient air, and $k_{\text {in }}=$ thermal conductivity of fiberglass insulation.

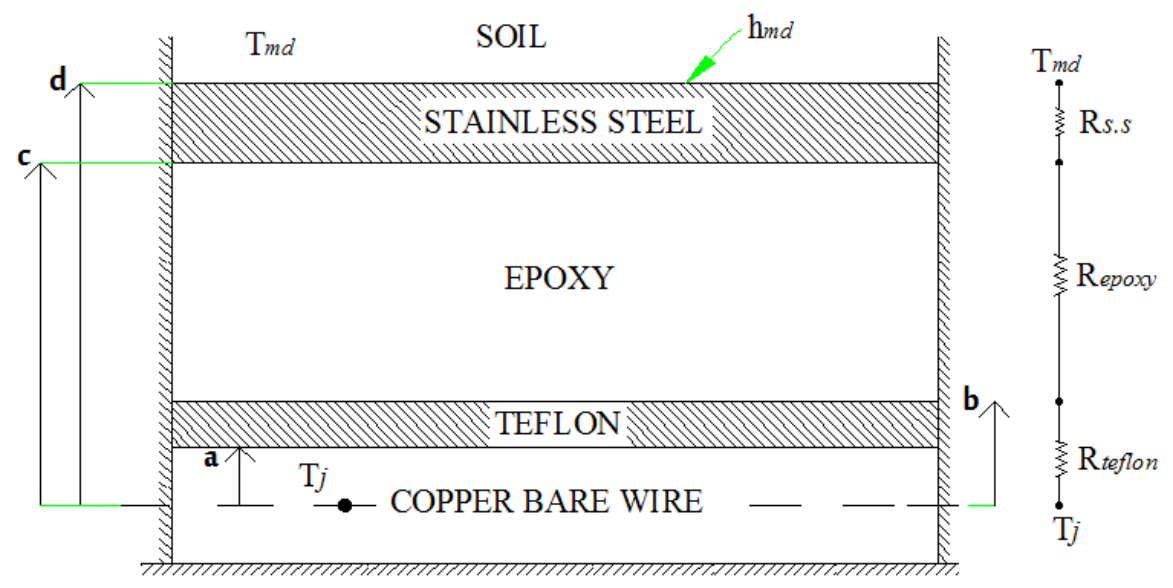

Fig. 4.2 Schematic of thermal resistance network between bare copper wire and soil medium

\subsubsection{Uncertainty Caused by Heat Conduction from Stainless Steel Needle and Coaxial Cable}

Conduction heat loss also occurs from the S.S needle to the threaded copper wire of the coaxial cable which is soldered to the S.S needle. The following equations describe the conduction loss in three different sections along the S.S needle and cable:

(i) Along T-TDR probe's S.S needle in the soil $\left(0 \leq x \leq L_{\text {needle }}\right)$ :

$\frac{\partial^{2} T}{\partial x^{2}}-\alpha_{m d}^{2}\left(T-T_{m d}\right)=0$

where

$\alpha_{m d}^{2}=\frac{h_{m d} P}{k_{s . S} A}=\frac{4 h_{m d} D_{2 s . s}}{k_{s . s}\left(D_{2 s . S}^{2}-D_{1 s . s}^{2}\right)}$

and 
$h_{m d}=h_{c}$

(ii) Along threaded copper wire of coaxial cable in insulation layer $\left(0 \leq x_{1} \leq L_{1}\right)$ :

$\frac{\partial^{2} T}{\partial x_{1}^{2}}-\alpha_{i n}^{2}\left(T-T_{a}\right)=0$

where

$\alpha_{\text {in }}^{2}=\frac{h_{\text {in }} P}{k_{\text {copper }} A}=\frac{4 h_{\text {in }} D_{2}}{k_{\text {copper }}\left(D_{2}^{2}-D_{1}^{2}\right)}$

and

$h_{\text {in }}=1 /\left(\frac{D_{2} \ln \frac{D_{3}}{D_{2}}}{k_{p v c}}+\frac{D_{2} \ln \frac{4 L_{1}}{D_{3}}}{k_{i n}}\right)$

(iii) Along threaded copper wire of coaxial cable in the ambient air $\left(0 \leq x_{2} \leq \infty\right)$ :

$\frac{\partial^{2} T}{\partial x_{2}^{2}}-\alpha_{a}^{2}\left(T-T_{a}\right)=0$

where

$\alpha_{a}^{2}=\frac{h_{a} P}{k_{\text {copper }} A}=\frac{4 h_{a} D_{2}}{k_{\text {copper }}\left(D_{2}^{2}-D_{1}^{2}\right)}$

and

$h_{a}=1 /\left(\frac{D_{2} \ln \frac{D_{3}}{D_{2}}}{2 k_{p v c}}+\frac{D_{2}}{h_{c o n v} D_{3}}\right)$

The $h_{\text {conv }}$ is natural convection heat transfer coefficient and can be computed by following equation (Incropera et al. 2007):

$h_{\text {conv }}=\left(\frac{k_{\text {air }}}{D_{3}}\right) \overline{N u}$ 
where Nusselt number can be calculated using Churchill and Chu equation (Incropera et al. 2007):

$\overline{N u}=\left\{0.6+\frac{0.387 R_{a}^{\left(\frac{1}{6}\right)}}{\left[1+\left(\frac{0.559}{P r}\right)^{\left(\frac{9}{16}\right)}\right)^{\frac{8}{27}}}\right\}^{2}$

for $\quad R a \leq 10^{12}$ and $R a \approx \frac{g\left(T_{m d}-T_{a}\right) D_{3}^{3}}{\left(T_{m d}\right) v \alpha}$

\subsubsection{Calculation of the Temperature Measurement Uncertainty}

Considering the following boundary conditions:

(1) Heat flux is negligible at the thermocouple's junction

$$
\left[\frac{d T}{d x}\right]_{x=0}=0
$$

(2) Temperature is continuous through the entire wire

$$
T_{x=L}=T_{x_{1}=0}, T_{x_{1}=L_{1}}=T_{x_{2}=0}
$$

(3) Heat flux is continuous through the entire wire

$$
\left[\frac{d T}{d x}\right]_{x=L}=\left[\frac{d T}{d x_{1}}\right]_{x_{1}=0},\left[\frac{d T}{d x_{1}}\right]_{x_{1}=L_{1}}=\left[\frac{d T}{d x_{2}}\right]_{x_{2}=0}
$$

(4) As $x_{2} \rightarrow \infty$ then $T \rightarrow T_{a}$

By solving Eqs. (4.26) through (4.33) with the boundary conditions defined above the temperature measurement uncertainty caused by the conduction heat loss can be determined as:

$T_{m d}-T_{j}=\frac{\left[e^{\alpha_{S} L_{1}}+\left(\frac{\alpha_{i n}}{\alpha_{a}}-1\right) \sinh \alpha_{i n} L_{1}\right]\left(T_{m d}-T_{a}\right)}{e^{\alpha_{S} L_{1} \beta+\left(\frac{\alpha_{i n}}{\alpha_{a}}-1\right) \gamma}}$

where 


$$
\beta=\cosh \alpha_{m d} L+\frac{\alpha_{m d}}{\alpha_{i n}} \sinh \alpha_{m d} L
$$

$\gamma=\cosh \alpha_{m d} L \sinh \alpha_{i n} L_{1}+\frac{\alpha_{m d}}{\alpha_{i n}} \sinh \alpha_{m d} L \cosh \alpha_{i n} L_{1}$

The maximum uncertainty can be assumed to be at $L_{1}=0$ when the wire is extended to the ambient without passing through insulation so that Eq. (4.36) can be written as:

$$
\left(T_{m d}-T_{j}\right)_{\max }=\frac{\left(T_{m d}-T_{a}\right)}{\cosh \alpha_{m d} L+\frac{\alpha_{m d} d}{\alpha_{a}} \sinh \alpha_{m d} L}
$$

The minimum uncertainty can be assumed when $L_{1} \rightarrow \infty$ meaning that the entire length of wire is in insulation layer, so that $\alpha_{a}=0$ and Eq. (4.36) can be written as:

$$
\left(T_{m d}-T_{j}\right)_{\min }=\frac{\left(T_{m d}-T_{a}\right)}{\cosh \alpha_{m d} L+\frac{\alpha_{m d} d}{\alpha_{i n}} \sinh \alpha_{m d} L}
$$

\subsubsection{Summary of the Uncertainty Calculations for Temperature Measurements}

Thermocouple wire used in this experiment consists copper and constantan; however, only copper wire was considered in the calculations since its thermal conductivity is significantly greater than constantan. The thermocouple-wire case is more severe than the stainless-steel tube case, even though the copper wire is so much smaller than the S.S. tube. However, the uncertainty for thermocouple-wire case is still negligible. The results from thermocouple-wire case for maximum and minimum uncertainty were found to be respectively $6.24 \times 10^{-6{ }^{\circ}} \mathrm{C}$ and $3.1 \times$ $10^{-7 \circ} \mathrm{C}$ and from stainless-steel tube case for maximum and minimum uncertainty were respectively $2.02 \times 10^{-15 \circ} \mathrm{C}$ and $2.83 \times 10^{-15 \circ} \mathrm{C}$. Therefore, it was concluded that although the uncertainty, caused by heat conduction through the wire, could have potentially significant impact on the experimental results, it was found to be negligible in this study. However, there is still systematic or bias uncertainty of $\pm 0.5^{\circ} \mathrm{C}$ or $0.4 \%$ (whichever is greater) due to T-type thermocouples ${ }^{12}$.

${ }^{12}$ TT-T-40-SLE OMEGA Thermocouple 
Table 4.6 Input data for uncertainty calculations due to conduction heat transfer

S.S. thermal conductivity, $k_{\text {s.s }}(\mathrm{W} / \mathrm{m} \cdot \mathrm{K})$

Epoxy thermal conductivity, $k_{\text {epoxy }}(\mathrm{W} / \mathrm{m} \cdot \mathrm{K})$

1.04

Copper thermal conductivity, $k_{\text {copper }}(\mathrm{W} / \mathrm{m} \cdot \mathrm{K})$

388

Insulation thermal conductivity, $k_{\text {in }}(\mathrm{W} / \mathrm{m} \cdot \mathrm{K})$

0.035

Teflon thermal conductivity, $k_{\text {teflon }}(\mathrm{W} / \mathrm{m} \cdot \mathrm{K})$

0.25

PVC thermal conductivity, $k_{p v c}(\mathrm{~W} / \mathrm{m} \cdot \mathrm{K})$

0.19

Soil thermal conductivity, $k_{s}(\mathrm{~W} / \mathrm{m} \cdot \mathrm{K})$

0.26

Diameter with threaded copper, D2 (m)

0.0042

Diameter without threaded copper, D1(m)

0.0036

Inner radius of PVC layer $(\mathrm{m})$

0.0021

PVC thickness (m)

0.0012

Outer diameter of PVC layer of coaxial cable, D3 (m)

0.0066

Radius of bare copper wire, a (m)

0.000045

Radius of copper wire with Teflon cover, $b(\mathrm{~m})$

0.000125

S.S. needle outer diameter, $\mathrm{D} 2 \mathrm{s.s}=2 \mathrm{~d}(\mathrm{~m})$

0.000127

S.S. needle inner diameter, $\mathrm{D} 1_{\mathrm{s} . \mathrm{s}}=2 \mathrm{c}(\mathrm{m})$

0.00084

Thermal contact conductance at S.S. needle-Soil interface, $h_{c}$

$\left(\mathrm{W} / \mathrm{m}^{2} \cdot \mathrm{K}\right)(\mathrm{Lam} 2013)$

7273

Distance of thermocouple junction embedded in S.S. needle, $L(\mathrm{~m})$

0.02

Insulation thickness covering coaxial cable, $L_{1}(\mathrm{~m})$

0.1

Ambient temperature, $T_{\mathrm{a}}\left({ }^{\circ} \mathrm{C}\right)$

22.5

Soil temperature, $T_{\mathrm{md}}\left({ }^{\circ} \mathrm{C}\right)$

27.7

Thermal diffusivity of air, $\alpha_{a}\left(\mathrm{~m}^{2} / \mathrm{s}\right)$

0.000023

kinematic viscosity of air, $v_{a}\left(\mathrm{~m}^{2} / \mathrm{s}\right)$

0.000016

Thermal conductivity of air, $k_{a}(\mathrm{~W} / \mathrm{m} \cdot \mathrm{K})$

0.026

Volumetric thermal expansion coefficient of air, $\beta\left(\mathrm{K}^{-1}\right) \quad 0.036$

Prandtl Number of air, Pr

0.71

Gravitational acceleration, $\mathrm{g}\left(\mathrm{m} / \mathrm{s}^{2}\right)$

9.81

Rayleigh number, Ra

1476

Nusselt number, $\mathrm{Nu}$

2.83

Natural convection heat transfer coefficient, $h_{\text {conv }}\left(\mathrm{W} / \mathrm{m}^{2} \cdot \mathrm{K}\right)$

22.6 


\subsection{Summary}

In this study, the analysis was carried out to estimate the uncertainties of the experimentally-determined quantities. The analysis was consisted of three main parts: 1Uncertainty analysis of heat pulse technique, 2- Uncertainty analysis of soil sample preparation and 3- Uncertainty analysis of temperature measurement via T-TDR Probe.

The computed uncertainties associated with the heat pulse technique in measuring the thermal properties and volumetric water content were implemented for the cases of dry, half saturated and saturated soil (Matilda) each at two different temperatures. The maximum uncertainty was found to be about $7.2 \%$ in the measurement of the thermal conductivity of the dry soil sample at $90^{\circ} \mathrm{C}$. The minimum uncertainty was found to be about $2 \%$ in the measurement of the volumetric heat capacity of saturated soil at $20^{\circ} \mathrm{C}$.

The overall uncertainties in sample preparation at $95 \%$ confidence level were determined for three types of soils. The three types of soils are Matilda (ON-3), Ottawa sand (C-190) and FSJ\#1 (BC-1). For each $\mathrm{S}_{\mathrm{r}}$ and soil type, the overall uncertainty of the soil sample preparation was evaluated based on ten soil samples $(n=10)$ with the student's $t$ multiplier of 2.262 for $95 \%$ confidence and nine degrees of freedom. It can be seen that the maximum overall uncertainty in soil sample preparation is about $2.7 \%$ when $\mathrm{S}_{\mathrm{r}}$ is low.

From the uncertainty analysis of temperature measurement via T-TDR Probe it was found that the thermocouple-wire case is more severe than the stainless-steel tube case, even though the copper wire is so much smaller than the S.S. tube. However, the uncertainty for thermocouplewire case is still negligible. The results from thermocouple-wire case for maximum and minimum uncertainty were found to be respectively $6.24 \times 10^{-6 \circ} \mathrm{C}$ and $3.1 \times 10^{-7 \circ} \mathrm{C}$ and from stainlesssteel tube case for maximum and minimum uncertainty were respectively $2.02 \times 10^{-15{ }^{\circ}} \mathrm{C}$ and $1.83 \times 10^{-15^{\circ}} \mathrm{C}$. Therefore, it was concluded that although the uncertainty, caused by heat conduction through the wire, could have potentially significant impact on the experimental results it found to be negligible in this study. 


\section{CHAPTER 5}

\section{EXPERIMENTAL EVALUATION OF TDR PROBES INTERFERENCE}

\subsection{Introduction}

The interference effect between two adjacent time domain reflectometry (TDR) probes has been evaluated and analyzed experimentally. In this experiment three different soil samples were examined. The first soil was Matilda soil (Schönenberger et al. and Tarnawski et al. 2012) from Ontario, Canada with identification code of ON-3 which has loamy sand texture (71\% sand, $25.4 \%$ silt and $3.6 \%$ clay) and has a porosity of 0.46 . The second soil was FSJ\# $1^{13}$ soil (Schönenberger et al. and Tarnawski et al. 2012) from British Colombia, Canada with identification code of BC-1 which has silty clay texture ( $0 \%$ sand, $58 \%$ silt and $42 \%$ clay) and has a porosity of 0.51 . The third soil was Ottawa sand (Tarnawski et al. 2009 and Tarnawski et al. 2013) from Ottawa, Illinois, USA with identification code of C-190 which is quartz sand (99.8\% sand and $0 \%$ clay) and has a porosity of 0.34 . For each soil, two samples of unsaturated soil and one saturated sample were packed into three separate glass containers (200 $\mathrm{mL}$ of volume each). Soil samples were prepared having degrees of saturation $\left(\mathrm{S}_{\mathrm{r}}\right)$ of $0.25,0.5$ and 1.0. The techniques which were applied to prepare the soil samples with specified degrees of saturation were taken from Tarnawski et al. (2015). Once a soil sample was ready, two TDR probes were inserted into the soil with a specified distance between them. The main objective of the study was to determine whether the reflected electromagnetic waveforms captured by the two probes would be mutually affected if the probes were placed in close vicinity to each other in the soil. In other words, it was desired to realize whether the two adjacent probes interfere with each other when they are operated simultaneously. Fig. 5.1 illustrates a schematic of experimental setup.

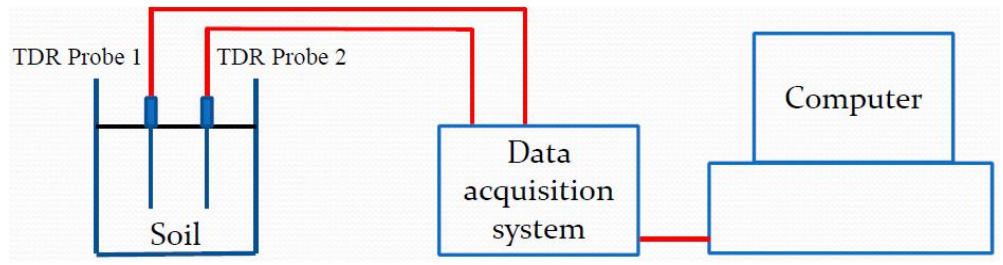

Fig. 5.1 Schematic of experimental setup

\footnotetext{
${ }^{13}$ Fort St. James is a district municipality and former fur trading post in north-central British Columbia, Canada.
} 


\subsection{Sample Preparation}

It was important to prepare all three types of soil samples in consistent ways so that the effect of interference on the TDR measurements would have been comparable among the samples. Three samples of the same soil, but with dissimilar $S_{r}$, were to be prepared. In the first step, a glass container with known volume (200 mL graded volume) was filled and evenly compacted with a known weight of dry soil so that a specific dry bulk density $\rho_{b}$ of the soil sample can be achieved (Tarnawski et al. 2015):

$\rho_{b}=\frac{m_{\text {soil }}}{V}$

where $m_{\text {soil }}$ is the mass of dry soil and $V$ is the volume of the glass container. Then, the porosity $\eta$ of the soil sample can be calculated using the following relation (Tarnawski et al. 2015):

$\eta=1-\frac{\rho_{b}}{\rho_{s}}$

where $\rho_{s}$ is the density of soil solids. The $\rho_{s}$ of ON-3, BC-1 and C-190 are $2706 \mathrm{~kg} / \mathrm{m}^{3}$ (Schönenberger et al. 2012), $2740 \mathrm{~kg} / \mathrm{m}^{3}$ (Schönenberger et al. 2012) and $2650 \mathrm{~kg} / \mathrm{m}^{3}$ (Tarnawski et al. 2009), respectively. The volume of air space within the soil texture can be determined by multiplying porosity with the volume of the sample, i.e. the volume of the glass container. The mass of required water to be mixed with the volume of the sample for $S_{\mathrm{r}}=0.25,0.5$ and 1.0 are respectively 25,50 and $100 \%$ of the air space volume.

$m_{w}=\rho_{w} \eta V S_{r}$

The preparation of soil samples follows the methodology provided by Tarnawski et al. (2015). There are four methods to be applied depending on soil texture and $\mathrm{S}_{\mathrm{r}}$. Method 2 (Mixing method) was applied to $\mathrm{ON}-3$ and BC-1 for the samples having $\mathrm{S}_{\mathrm{r}}$ of 0.25 and 0.5 ; however, for C-190 with $S_{\mathrm{r}}=0.25$ and 0.5, Method 2 (Mixing method) and Method 3 (Microwave Oven method) were applied, respectively. For all completely saturated samples $\left(\mathrm{S}_{\mathrm{r}}=1\right)$, Method 4 (Vacuum Pump method) was applied. Readers are encouraged to refer to Tarnawski et al. (2015) for details of the soil sample preparation methods. Fig. 5.2 illustrates two cases of TDR probes inserted in a soil sample of C-190. The right figure shows the position of the two probes when the distance between 
them is $1 \mathrm{~cm}$. The left photograph shows the position of the two probes when the distance between them is $4.2 \mathrm{~cm}$.

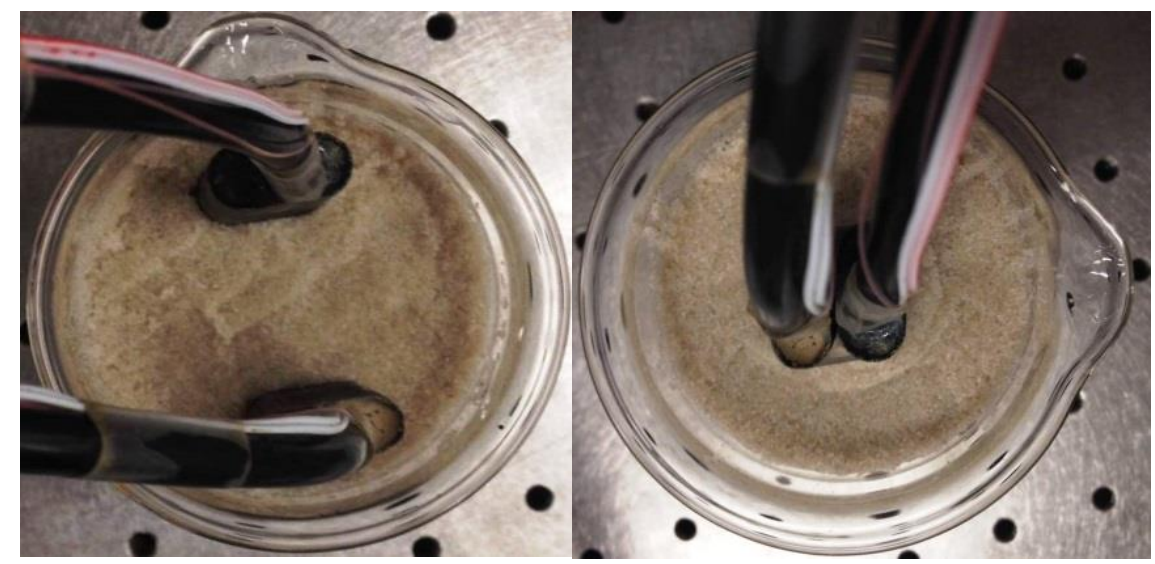

Fig. 5.2 Soil sample C-190 with inserted TDR probes at $1 \mathrm{~cm}$ and $4.2 \mathrm{~cm}$ apart

\subsection{Analysis of Results}

The effect of interference between two adjacent TDR probes was examined. A total of nine samples of $\mathrm{ON}-3, \mathrm{BC}-1$ and $\mathrm{C}-190$ soils with three different $\mathrm{S}_{\mathrm{r}}$ of $0.25,0.5$ and 1.0 were considered. When each probe was tested separately, the maximum uncertainty of 18 tests (i.e., three $S_{\mathrm{r}} \times$ three soil types $\times$ two TDR probes) in determining the volumetric water content (using Eqs. 2.3 and 2.4) was obtained to be $-4.91 \%$ for $\mathrm{ON}-3$ soil with $\mathrm{S}_{\mathrm{r}}=0.5$. Therefore, it can be established that the uncertainties of the TDR measurements in the present study are within $\pm 5 \%$. For the interference tests, the two probes were placed apart at four different distances parallel to and facing each other in vertical alignment. The distances were 4.2, 3.2, 2.2 and $1.0 \mathrm{~cm}$. In order to establish the overall uncertainty of soil sample preparation, the systematic $\left(B_{\theta}\right)$ uncertainty and precision $\left(S_{\bar{\theta}}\right)$ uncertainty of the mean volumetric water content $\bar{\theta}$ of ten $(M=10)$ independently prepared soil samples of $\theta_{k}(k=1,2, \ldots, M)$ at each $\mathrm{S}_{\mathrm{r}}$ for each soil type were evaluated. Then the uncertainties were combined using the root sum square (RSS) method to obtain the overall uncertainty at $95 \%$ confidence level (see Chapter 4 ). 


\subsubsection{Matilda}

Fig. 5.3 illustrates the waveforms that were captured by the probes when the distances between them were 2.2, 3.2 and $4.2 \mathrm{~cm}$ for $\mathrm{ON}-3$ and $\mathrm{S}_{\mathrm{r}}=0.25\left(\theta=0.116 \mathrm{~m}^{3} / \mathrm{m}^{3} \pm 1.72 \%\right.$ at $95 \%$ confidence level). It can be perceived that there is no interference since both waveforms are overlapping each other. The water contents determined by the two probes were 0.1136 and 0.1106 $\mathrm{m}^{3} / \mathrm{m}^{3}$, which are -2.07 and $-4.66 \%$ uncertainties comparing with the known $\theta$ of $0.116 \mathrm{~m}^{3} / \mathrm{m}^{3}$. However, when the distance was reduced to $1.0 \mathrm{~cm}$, some interference was observed as shown in Fig. 5.4. By looking at the waveforms in Fig. 5.4, it can be seen that the entire reflected waveform for probe 1 is slightly shifted to the right. Although the waveform was shifted, the apparent lengths of the two probes were similar; therefore, the determined water contents were 0.1135 and 0.1182 $\mathrm{m}^{3} / \mathrm{m}^{3}$, which are -2.16 and $+1.90 \%$ uncertainties.

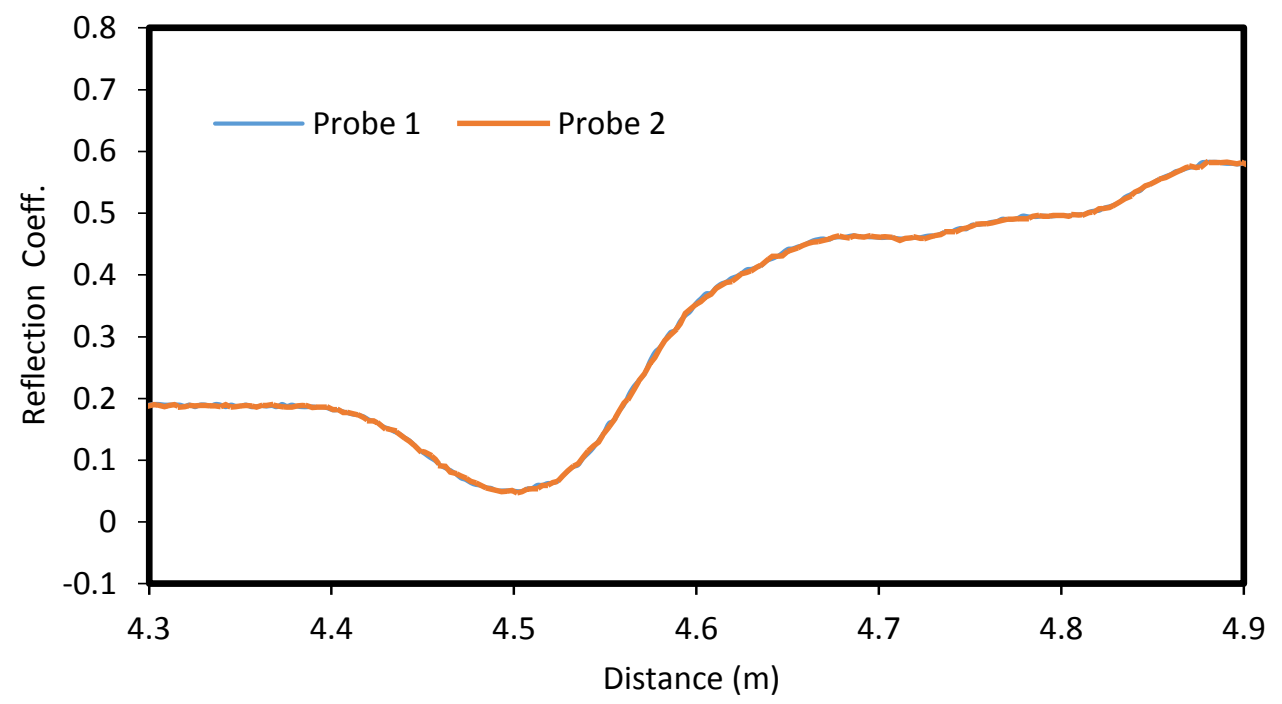

Fig. 5.3 The waveforms captured simultaneously by the probes placing 2.2, 3.2 and $4.2 \mathrm{~cm}$ apart in $\mathrm{ON}-3$ with $\mathrm{S}_{\mathrm{r}}=0.25$ 


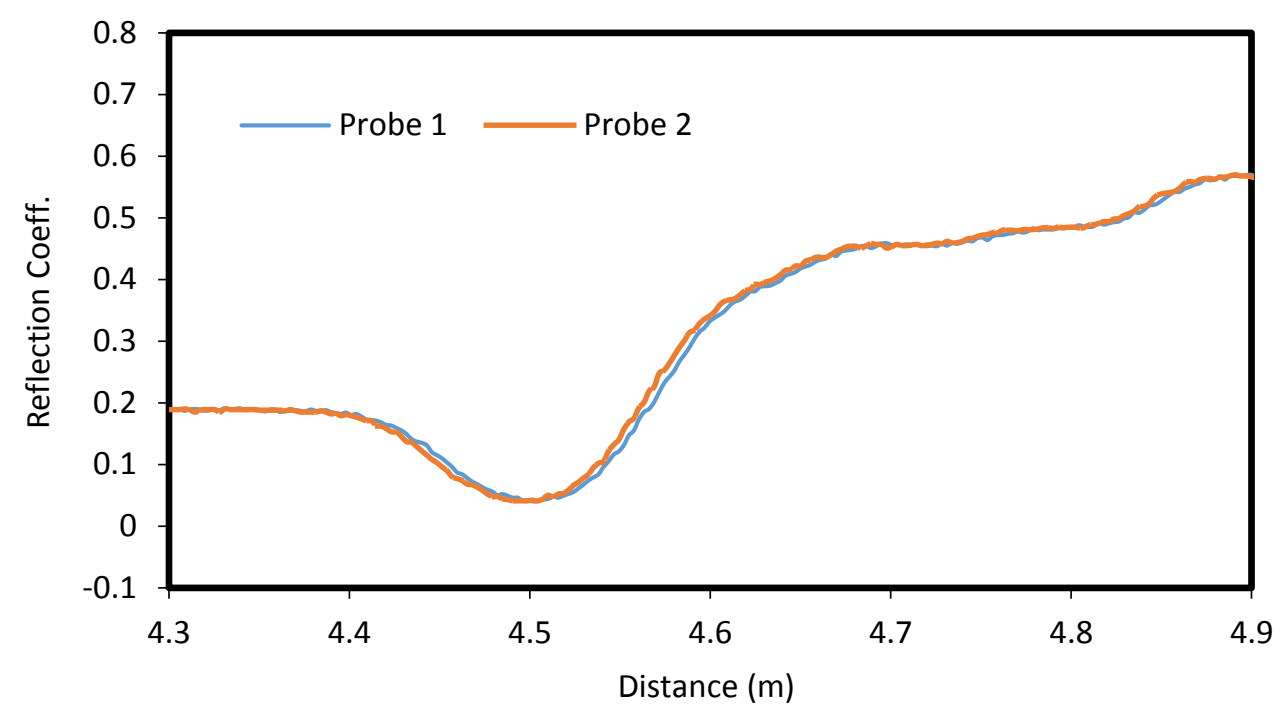

Fig. 5.4 The waveforms captured simultaneously by the probes placing $1.0 \mathrm{~cm}$ apart in $\mathrm{ON}-3$ with $S_{\mathrm{r}}=0.25$

Fig. 5.5 illustrates the waveforms that were captured by the probes when the distances between them were 2.2, 3.2 and $4.2 \mathrm{~cm}$ for ON-3 and $\mathrm{S}_{\mathrm{r}}=0.5\left(\theta=0.230 \mathrm{~m}^{3} / \mathrm{m}^{3} \pm 1.64 \%\right.$ at $95 \%$ confidence level). It can be perceived that there is negligible interference since both waveforms are essentially the same with the entire waveform of probe 1 being very slightly shifted to the right. The water contents determined by the two probes were 0.2187 and $0.2394 \mathrm{~m}^{3} / \mathrm{m}^{3}$, which are -4.91 and $+4.09 \%$ uncertainties comparing with the known $\theta$ of $0.230 \mathrm{~m}^{3} / \mathrm{m}^{3}$. However, when the distance was reduced to $1.0 \mathrm{~cm}$, some interference was observed as shown in Fig. 5.6. By looking at the waveforms in Fig. 5.6, it can be seen that the entire reflected waveform for probe 1 is slightly shifted to the right. Although the waveform was shifted, the apparent lengths of the two probes were similar; therefore, the determined water contents were 0.2193 and $0.2318 \mathrm{~m}^{3} / \mathrm{m}^{3}$, which are -4.65 and $+0.78 \%$ uncertainties. 


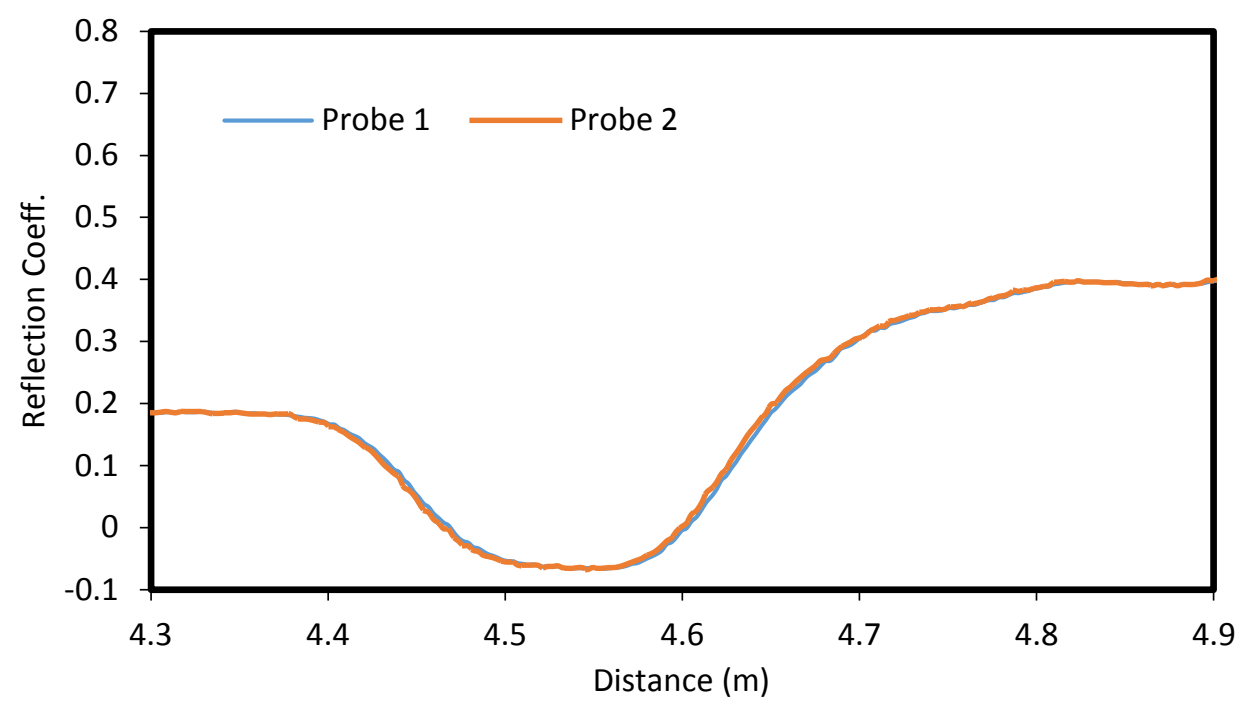

Fig. 5.5 The waveforms captured simultaneously by the probes placing 2.2, 3.2 and $4.2 \mathrm{~cm}$ apart in $\mathrm{ON}-3$ with $\mathrm{S}_{\mathrm{r}}=0.5$

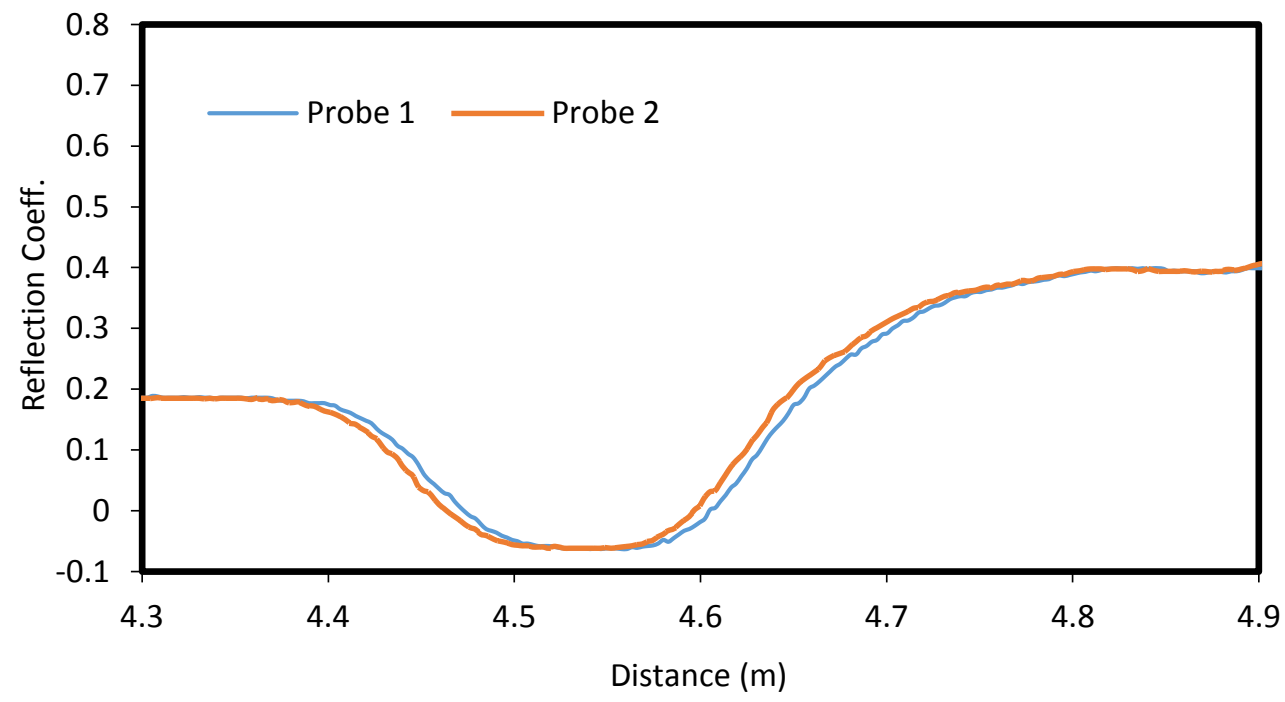

Fig. 5.6 The waveforms captured simultaneously by the probes placing $1.0 \mathrm{~cm}$ apart in $\mathrm{ON}-3$ with $\mathrm{S}_{\mathrm{r}}=0.5$ 
Fig. 5.7 illustrates the waveforms that were captured by the probes when the distances between them were 2.2, 3.2 and $4.2 \mathrm{~cm}$ for $\mathrm{ON}-3$ and $\mathrm{S}_{\mathrm{r}}=1.0\left(\theta=0.460 \mathrm{~m}^{3} / \mathrm{m}^{3} \pm 1.51 \%\right.$ at $95 \%$ confidence level). It can be perceived that there is again negligible interference since both waveforms are essentially the same with the entire waveform of probe 1 being very slightly shifted to the right. The water contents determined by the two probes were 0.4658 and $0.4588 \mathrm{~m}^{3} / \mathrm{m}^{3}$, which are +1.26 and $-0.26 \%$ uncertainties comparing with the known $\theta$ of $0.460 \mathrm{~m}^{3} / \mathrm{m}^{3}$. However, when the distance was reduced to $1.0 \mathrm{~cm}$, some interference was observed as shown in Fig. 5.8. By looking at the waveforms in Fig. 5.8, it can be seen that the latter half of the reflected waveform for probe 1 is slightly shifted downward and to the right. This uneven shift caused the apparent lengths of the two probes to be dissimilar; therefore, the determined water contents were 0.4573 and $0.4387 \mathrm{~m}^{3} / \mathrm{m}^{3}$, which are -0.59 and $-4.63 \%$ uncertainties.

Comparing the waveforms shown in Figs. 5.3, 5.5 and 5.7, it can be realized that the increase of water content in ON-3 would induce negligible interference effect, when the distances between probes were 2.2,3.2 and $4.2 \mathrm{~cm}$; the analysis of these waveforms results in the same water content values for the two probes. The increase of water content would change the features of the waveform. That is, as the water content increases, the reflected waveform would be deeper and wider, resulting in longer apparent length of the probe or higher dielectric constant of the soil.

Figs. 5.4, 5.6 and 5.8 illustrate the waveforms that were captured by the probes when the distance between them was $1.0 \mathrm{~cm}$ for ON-3. It can be seen that the severity of interference increases as the water content increases. Up to $S_{r}=0.5$, the interference only causes horizontal shift of the waveforms without altering the results. However, at fully saturated condition $\left(\mathrm{S}_{\mathrm{r}}=1\right)$, the interference affects features of the waveform, resulting in different results and relatively high uncertainty. By comparing with the previous observations, it can be concluded that both water content and distance between the probes influence the interference effect. It was determined that for ON-3 the interference, as a result of water content increase up to $\theta=0.230 \mathrm{~m}^{3} / \mathrm{m}^{3}$, would shift the position of the electromagnetic wave reflections over the entire waveform but it would not affect the probe's capability of correct measurement. However, when ON-3 was in fully saturated condition $\left(\theta=0.460 \mathrm{~m}^{3} / \mathrm{m}^{3}\right)$ and the distance between probes was reduced to $1.0 \mathrm{~cm}$, as shown in Fig. 5.8, the accuracy of TDR measurements was affected by the interference; but the uncertainties were still within the $5 \%$. This seems to indicate that for medium coarse soil (loamy sand) the effect 
of interference is not significant even when the water content is high and the distance between probes is short.

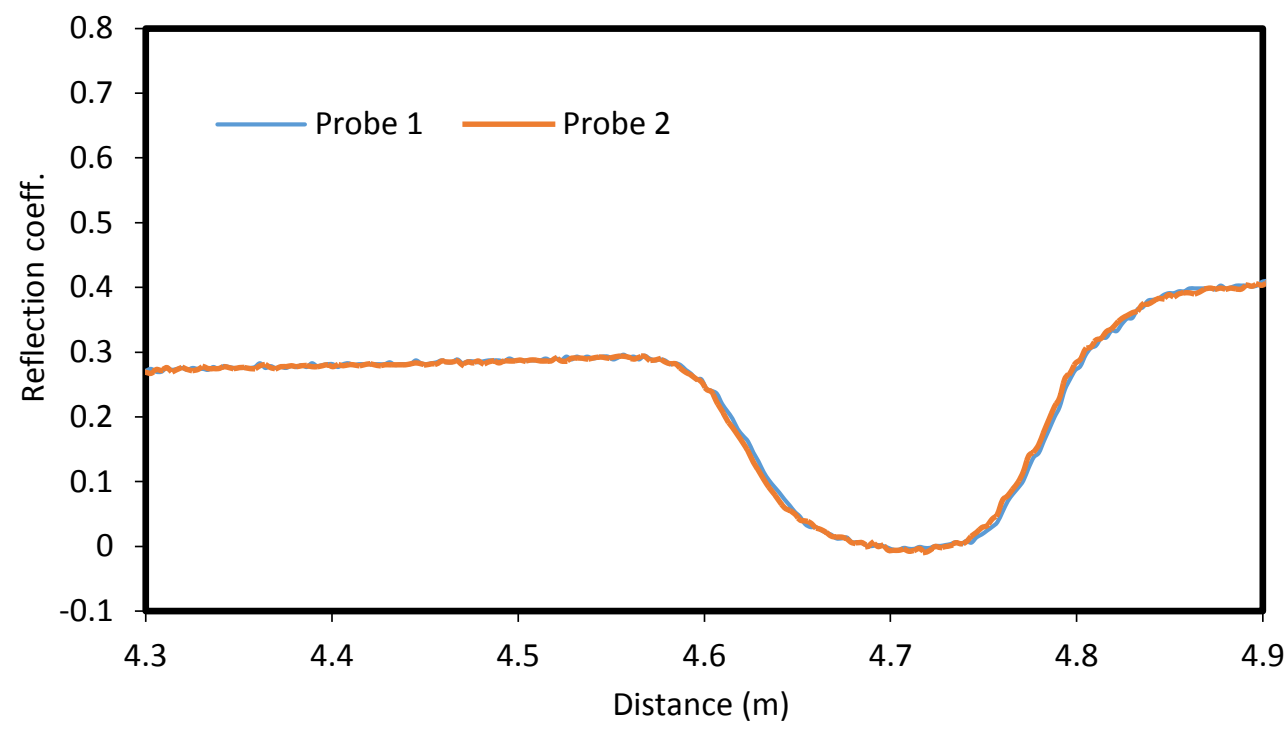

Fig. 5.7 The waveforms captured simultaneously by the probes placing 2.2, 3.2 and $4.2 \mathrm{~cm}$ apart in $\mathrm{ON}-3$ with $\mathrm{S}_{\mathrm{r}}=1$

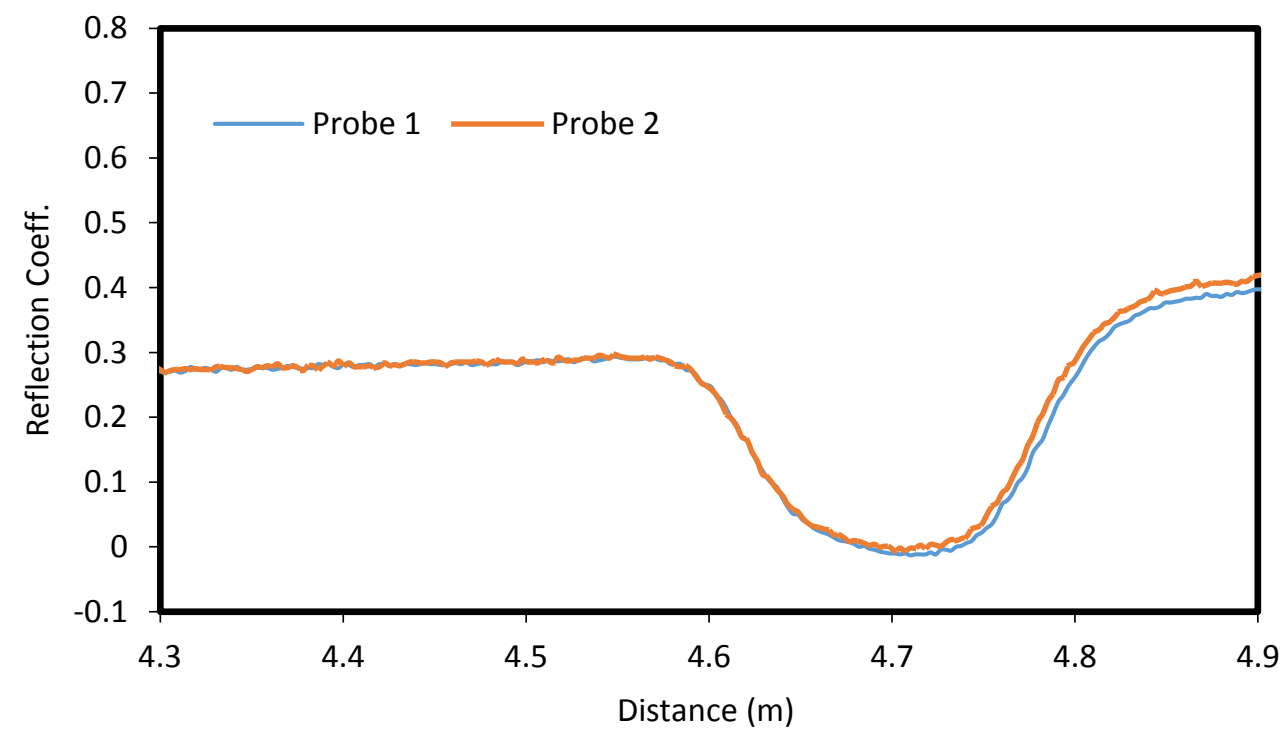

Fig. 5.8 The waveforms captured simultaneously by the probes placing $1.0 \mathrm{~cm}$ apart in $\mathrm{ON}-3$ with $S_{\mathrm{r}}=1$ 


\subsubsection{FSJ \# 1}

Fig. 5.9 illustrates the waveforms that were captured by the probes when the distances between them were 2.2, 3.2 and $4.2 \mathrm{~cm}$ for BC-1 and $\mathrm{S}_{\mathrm{r}}=0.25\left(\theta=0.127 \mathrm{~m}^{3} / \mathrm{m}^{3} \pm 2.42 \%\right.$ at $95 \%$ confidence level). It can be perceived that there is no interference since both waveforms are overlapping each other. The water contents determined by the two probes were 0.1232 and 0.1216 $\mathrm{m}^{3} / \mathrm{m}^{3}$, which are -2.99 and $-4.25 \%$ uncertainties comparing with the known $\theta$ of $0.127 \mathrm{~m}^{3} / \mathrm{m}^{3}$. However, when the distance was reduced to $1.0 \mathrm{~cm}$, some interference was observed as shown in Fig. 5.10. By looking at the waveforms in Fig. 5.10, it can be noticed that not only the entire reflected waveform for probe 2 is slightly shifted to the right but also the configuration of the waveforms for both probes was altered; hence, the apparent lengths were distorted. Therefore, unlike the previous case the uncertainties in measurements were increased as a result of interference. The determined water contents were 0.1158 and $0.1152 \mathrm{~m}^{3} / \mathrm{m}^{3}$, which are -8.82 and $-9.29 \%$ uncertainties.

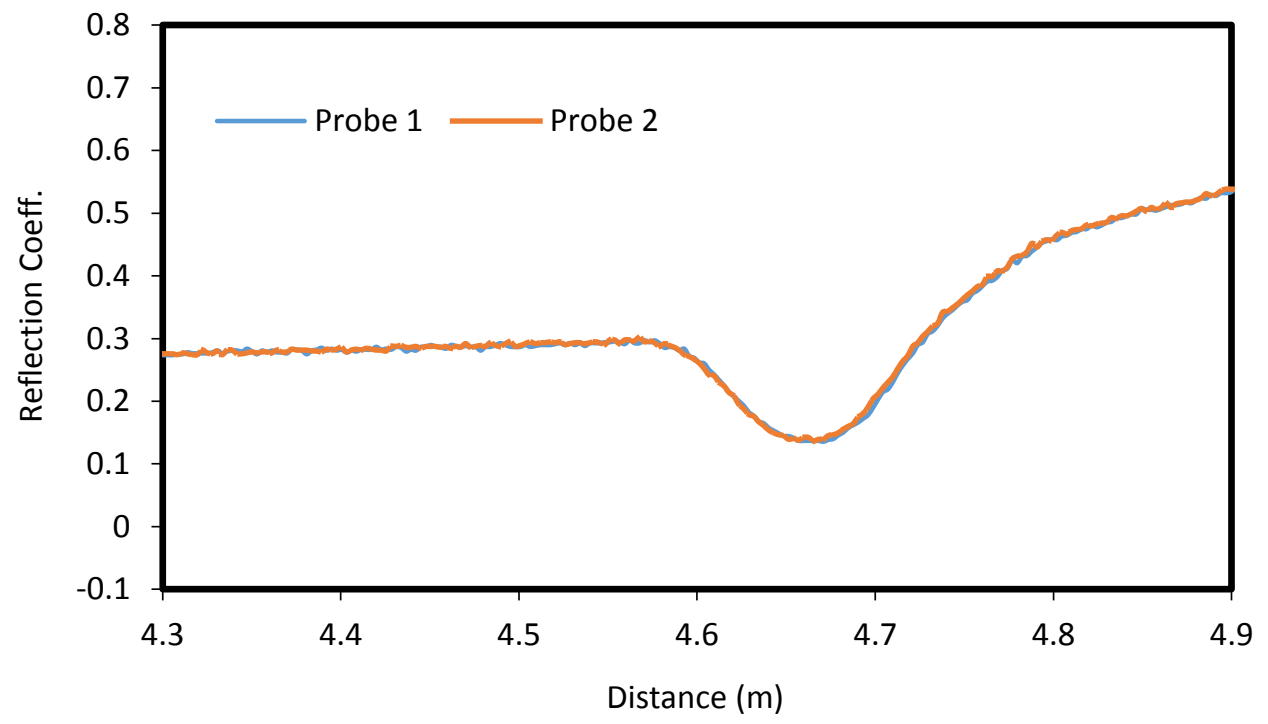

Fig. 5.9 The waveforms captured simultaneously by the probes placing 2.2, 3.2 and $4.2 \mathrm{~cm}$ apart in BC-1 soil with $\mathrm{S}_{\mathrm{r}}=0.25$ 


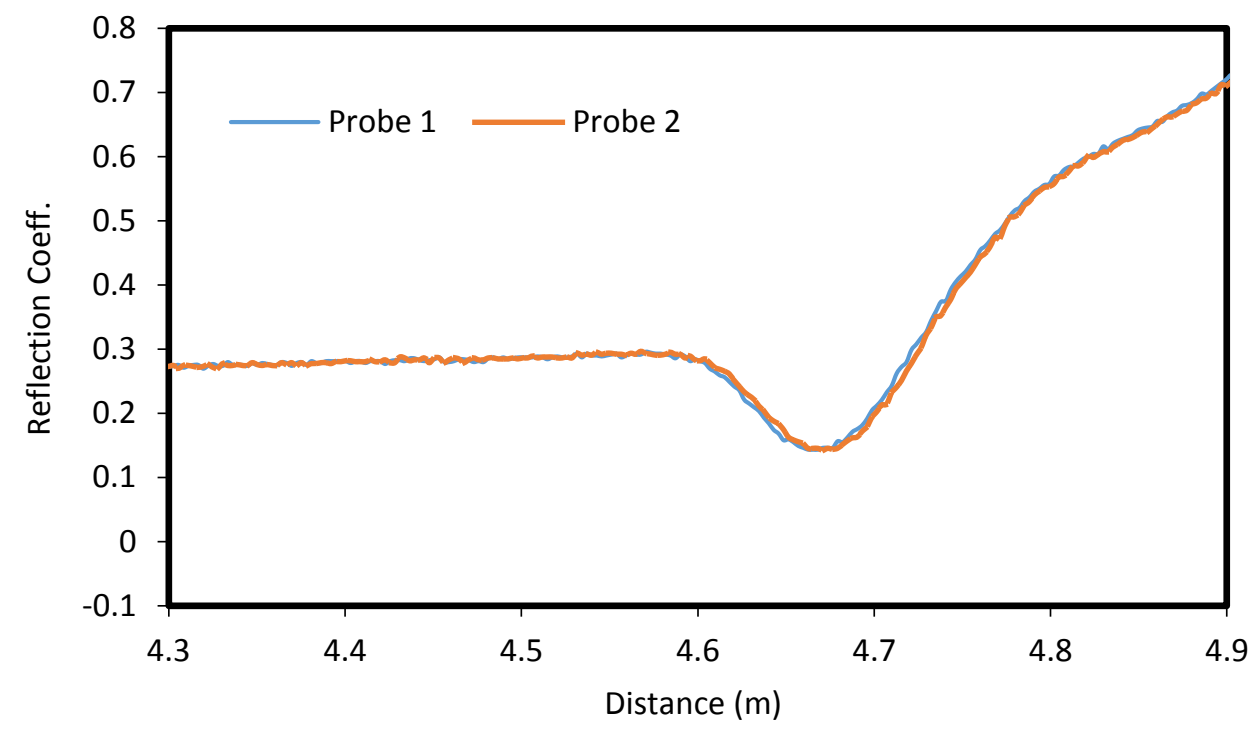

Fig. 5.10 The waveforms captured simultaneously by the probes placing $1.0 \mathrm{~cm}$ apart in $\mathrm{BC}$ 1soil with $\mathrm{S}_{\mathrm{r}}=0.25$

Fig. 5.11 illustrates the waveforms that were captured by the probes when the distances between them were $2.2,3.2$ and $4.2 \mathrm{~cm}$ for $\mathrm{BC}-1$ and $\mathrm{S}_{\mathrm{r}}=0.5\left(\theta=0.255 \mathrm{~m}^{3} / \mathrm{m}^{3} \pm 1.81 \%\right.$ at $95 \%$ confidence level). It can be perceived that there is no interference since both waveforms are overlapping each other. The water contents determined by the two probes were 0.2444 and 0.2481 $\mathrm{m}^{3} / \mathrm{m}^{3}$, which are -4.16 and $-2.71 \%$ uncertainties comparing with the known $\theta$ of $0.255 \mathrm{~m}^{3} / \mathrm{m}^{3}$. However, when the distance was reduced to $1.0 \mathrm{~cm}$, interference was observed as shown in Fig. 5.12. By looking at the waveforms in Fig. 5.12, it can be noticed that not only the entire reflected waveform for probe 1 is slightly shifted to the right but also the pattern of the waveforms for both probes was altered; hence, the apparent lengths were changed. Therefore, the uncertainties in measurements were increased as a result of interference. The determined water contents were 0.2223 and $0.2213 \mathrm{~m}^{3} / \mathrm{m}^{3}$, which are -12.8 and $-13.2 \%$ uncertainties. 


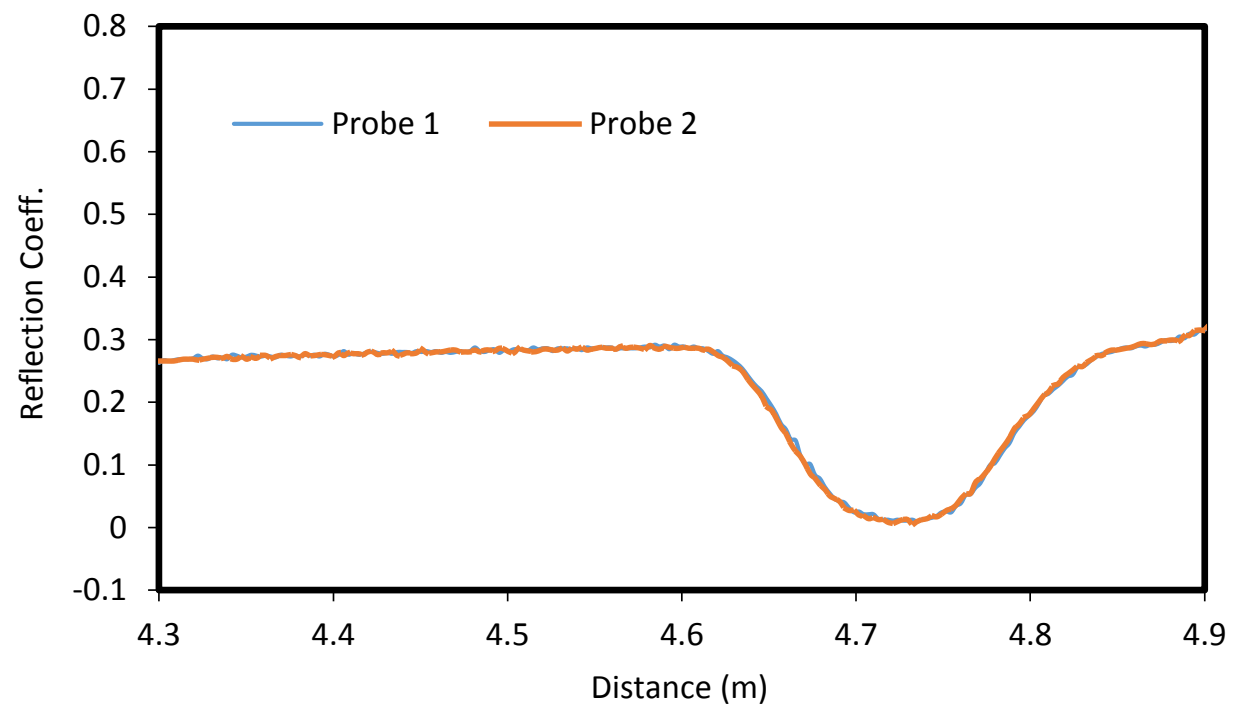

Fig. 5.11 The waveforms captured simultaneously by the probes placing $2.2,3.2$ and $4.2 \mathrm{~cm}$ apart in BC-1 soil with $\mathrm{S}_{\mathrm{r}}=0.5$

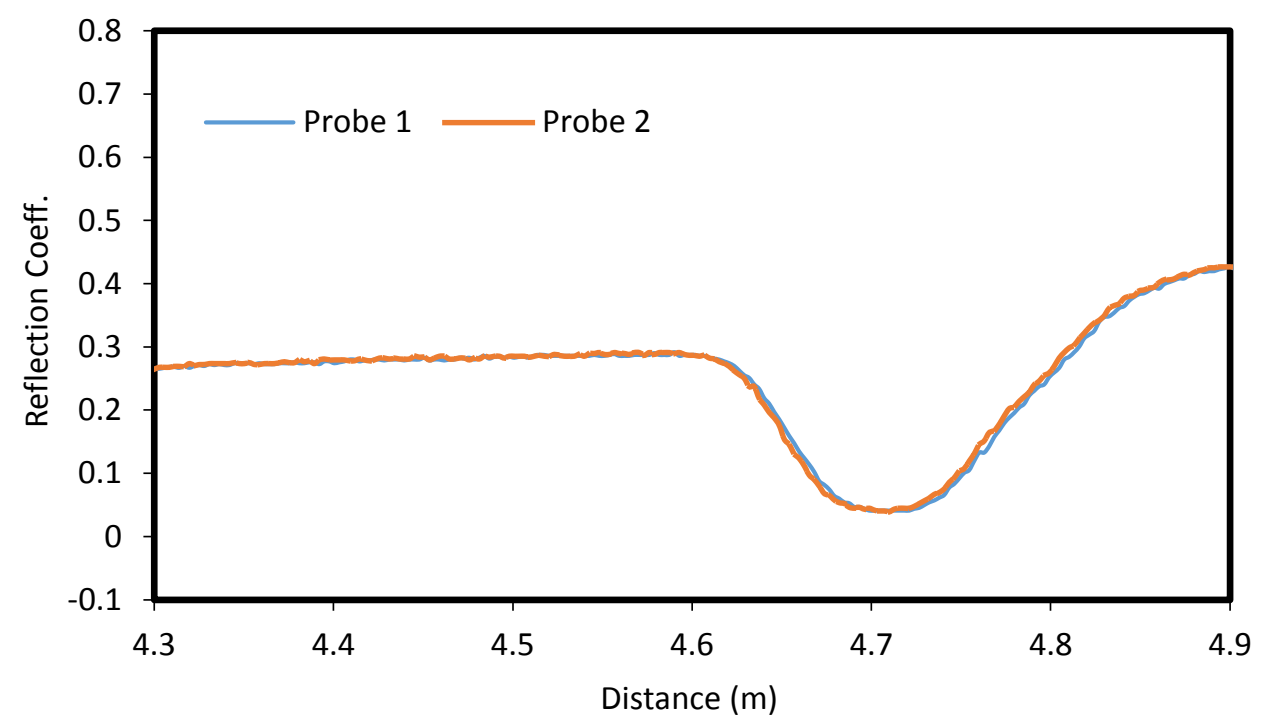

Fig. 5.12 The waveforms captured simultaneously by the probes placing $1.0 \mathrm{~cm}$ apart in $\mathrm{BC}-1$ soil with $\mathrm{S}_{\mathrm{r}}=0.5$ 
Fig. 5.13 illustrates the waveforms that were captured by the probes when the distances between them were 2.2, 3.2 and $4.2 \mathrm{~cm}$ for $\mathrm{BC}-1$ and $\mathrm{S}_{\mathrm{r}}=1.0\left(\theta=0.510 \mathrm{~m}^{3} / \mathrm{m}^{3} \pm 1.69 \%\right.$ at $95 \%$ confidence level). It can be perceived that there is again no interference since both waveforms are overlapping each other. The water contents determined by the two probes were 0.5175 and 0.5225 $\mathrm{m}^{3} / \mathrm{m}^{3}$, which are +1.47 and $+2.45 \%$ uncertainties comparing with the known $\theta$ of $0.510 \mathrm{~m}^{3} / \mathrm{m}^{3}$. However, when the distance was reduced to $1.0 \mathrm{~cm}$, some interference was observed as shown in Fig. 5.14. By looking at the waveforms in Fig. 5.14, it can be observed that not only the whole reflected waveform for probe 2 is shifted to the right but also the profile of the waveforms for both probes was changed; hence, the apparent lengths were changed. Thus, the uncertainties in measurements were increased as a result of interference. The determined water contents were 0.4323 and $0.4311 \mathrm{~m}^{3} / \mathrm{m}^{3}$, which are -15.2 and $-15.5 \%$ uncertainties.

Comparing the waveforms shown in Figs. 5.9, 5.11 and 5.13, it can be realized that increase of water content in BC-1 would induce no interference effect, when the distances between probes were 2.2, 3.2 and $4.2 \mathrm{~cm}$; the analysis of these waveforms results in the same water content values for the two probes. The increase of water content would change the features of the waveform. That is, as the water content increases, the reflected waveform would be deeper and wider, resulting in longer apparent length of the probe or higher dielectric constant of the soil.

Figs. 5.10, 5.12 and 5.14 illustrate the waveforms that were captured by the probes when the distance between them was $1.0 \mathrm{~cm}$ for BC-1. It can be seen that the severity of interference increases as the water content increases. For all cases, the interference affects features of the waveform, resulting in different results and relatively high uncertainty. By comparing with the previous observations, it can be concluded that both water content and distance between the probes influence the interference effect. It was determined that for BC-1 no interference occur, as a result of water content increase, when the distance between probes was greater than $1 \mathrm{~cm}$. However, when distance between probes was reduced to $1.0 \mathrm{~cm}$, as shown in Figs. 5.10, 5.12 and 5.14, the accuracy of TDR measurements was noticeably affected by the interference and uncertainties were increased as a result of water content increase from about $9 \%$ for $S_{r}=0.25$ to $15 \%$ for $S_{r}=1$. It is obvious that for the probes with $1-\mathrm{cm}$ distance in fine soil (silty clay) the effect of interference exists even when the water content is low $\left(S_{r}=0.25\right)$. 


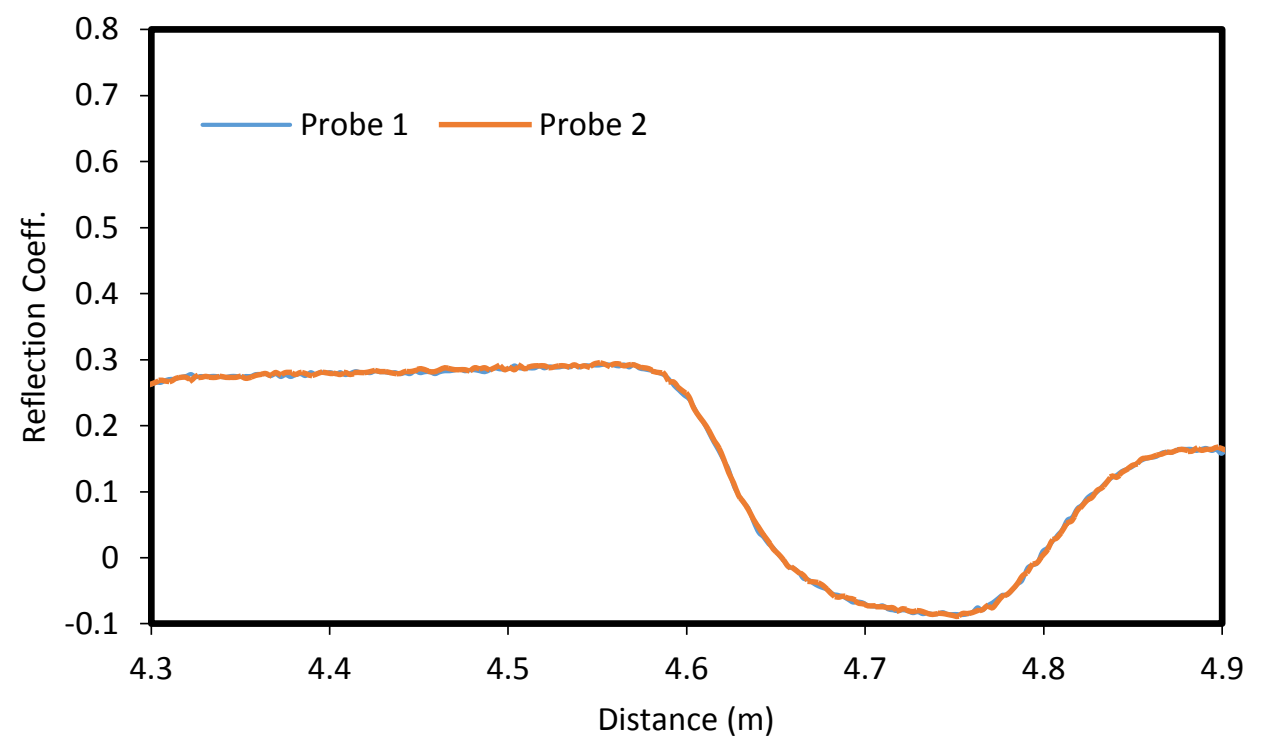

Fig. 5.13 The waveforms captured simultaneously by the probes placing 2.2, 3.2 and $4.2 \mathrm{~cm}$ apart in $\mathrm{BC}-1$ soil with $\mathrm{S}_{\mathrm{r}}=1$

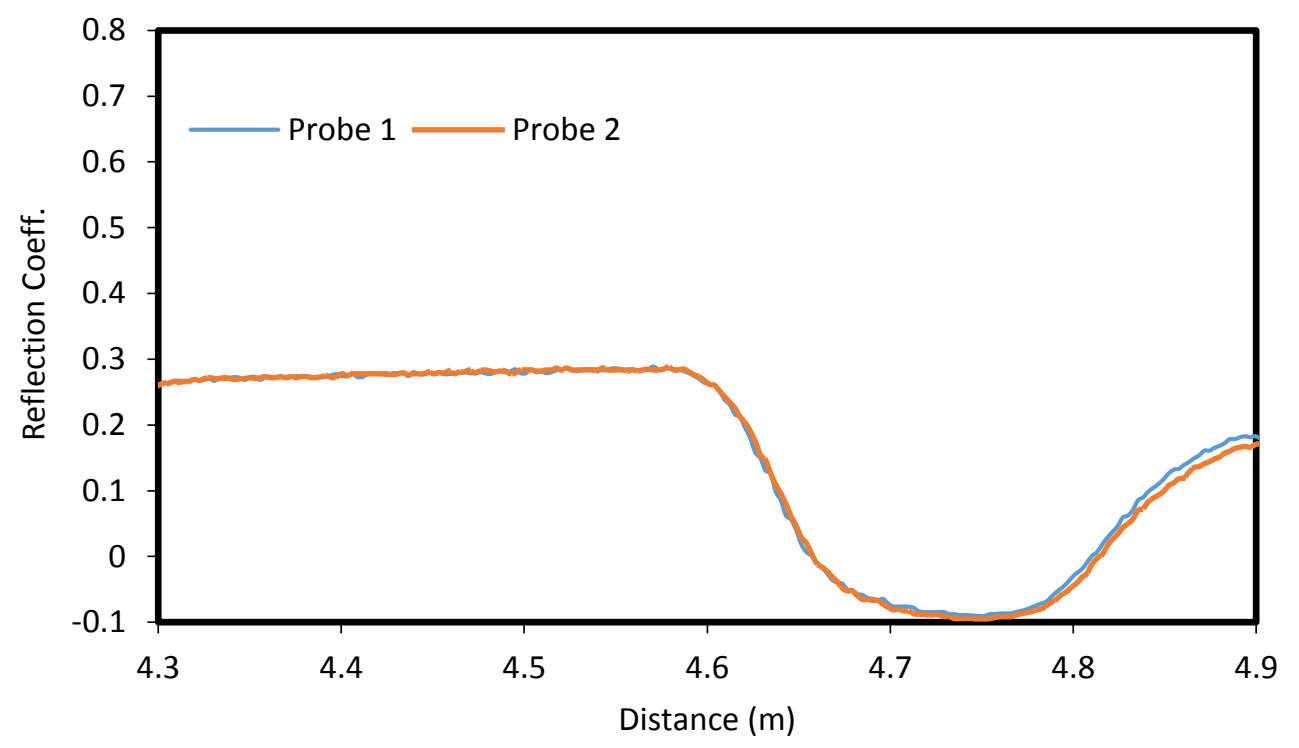

Fig. 5.14 The waveforms captured simultaneously by the probes placing $1.0 \mathrm{~cm}$ apart in $\mathrm{BC}-1$ soil with $\mathrm{S}_{\mathrm{r}}=1$ 


\subsubsection{Ottawa Sand}

Fig. 5.15 illustrates the waveforms that were captured by the probes when the distances between them were $2.2,3.2$ and $4.2 \mathrm{~cm}$ for $\mathrm{C}-190$ and $\mathrm{S}_{\mathrm{r}}=0.25\left(\theta=0.085 \mathrm{~m}^{3} / \mathrm{m}^{3} \pm 2.67 \%\right.$ at $95 \%$ confidence level). It can be perceived that there is no interference since both waveforms are overlapping each other. The water contents determined by the two probes were 0.0862 and 0.0858 $\mathrm{m}^{3} / \mathrm{m}^{3}$, which are +1.41 and $+0.94 \%$ uncertainties comparing with the known $\theta$ of $0.085 \mathrm{~m}^{3} / \mathrm{m}^{3}$. However, when the distance was reduced to $1.0 \mathrm{~cm}$, some interference was observed as shown in Fig. 5.16. By looking at the waveforms in Fig. 5.16, it can be seen that the entire reflected waveform for probe 2 is slightly shifted to the right. Although the waveform was shifted, the apparent lengths of the two probes were similar; therefore, the determined water contents were 0.0829 and $0.0866 \mathrm{~m}^{3} / \mathrm{m}^{3}$, which are -2.47 and $+1.88 \%$ uncertainties.

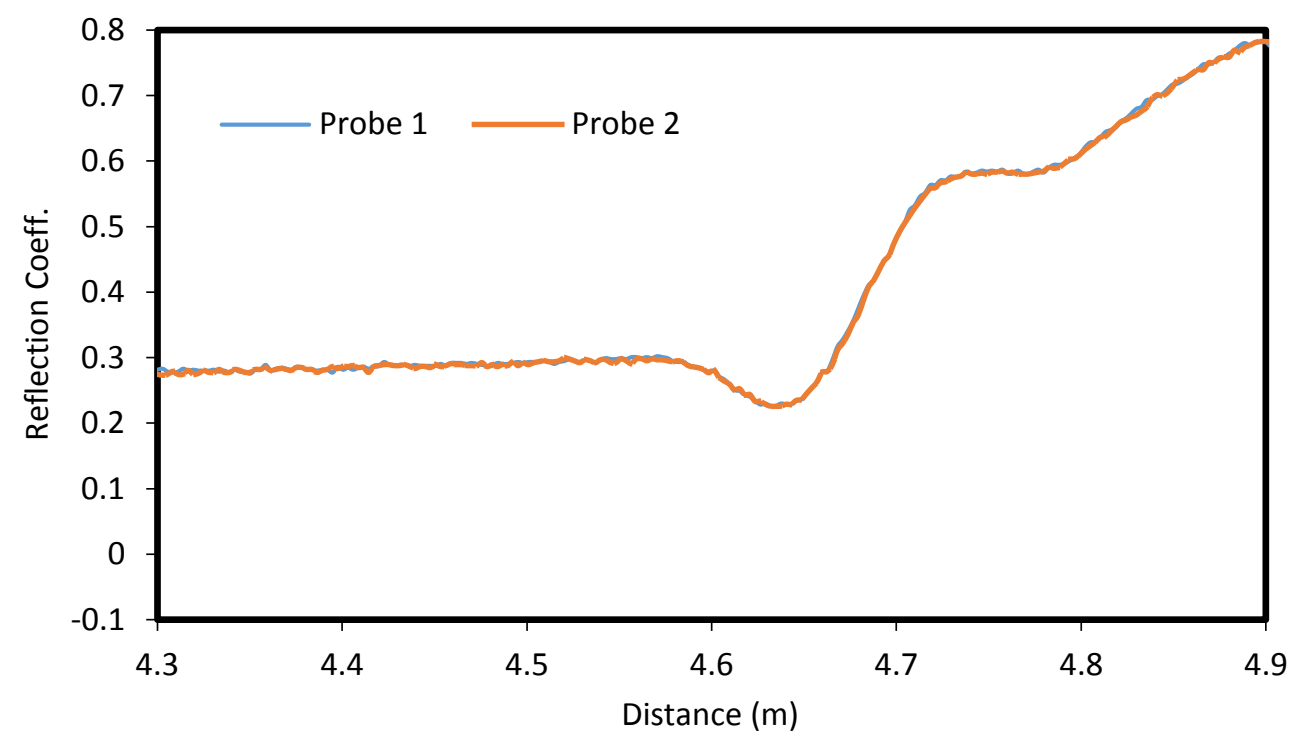

Fig. 5.15 The waveforms captured simultaneously by the probes placing 2.2, 3.2 and $4.2 \mathrm{~cm}$ apart in C-190 soil with $\mathrm{S}_{\mathrm{r}}=0.25$ 


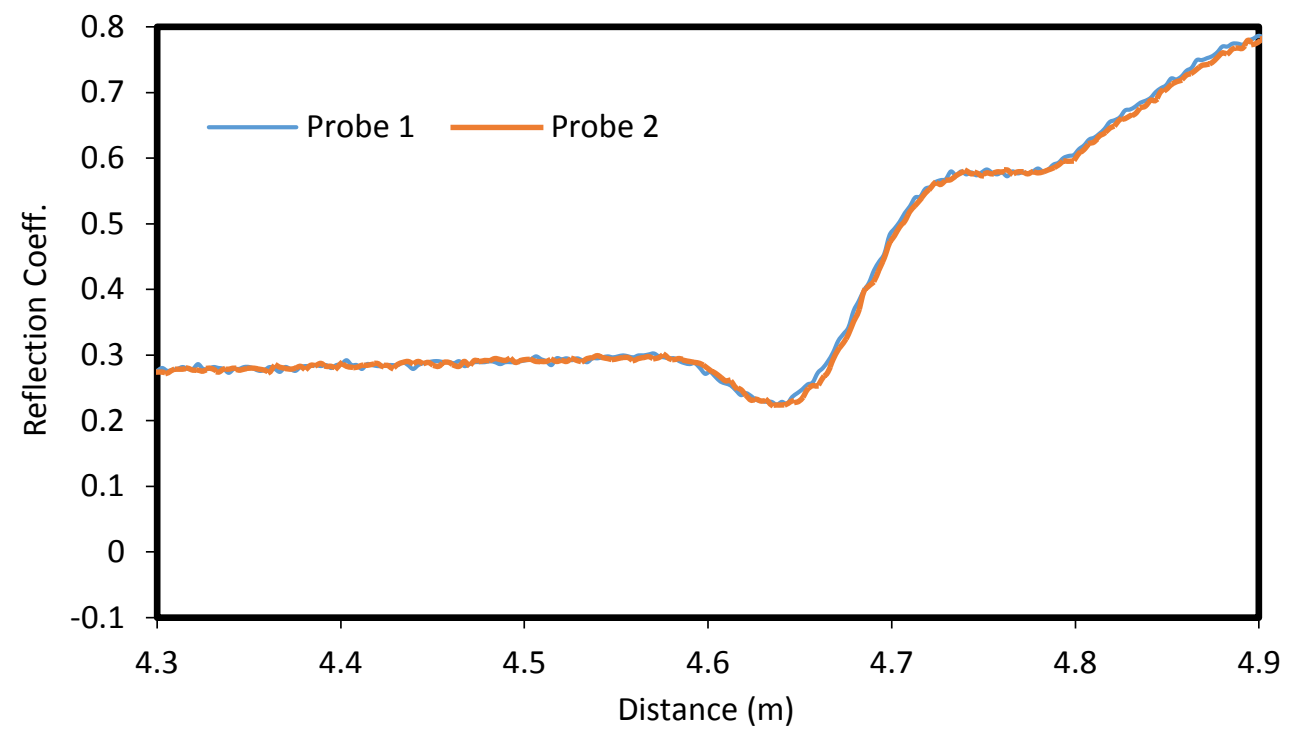

Fig. 5.16 The waveforms captured simultaneously by the probes placing $1.0 \mathrm{~cm}$ apart in C-190 soil with $\mathrm{S}_{\mathrm{r}}=0.25$

Fig. 5.17 illustrates the waveforms that were captured by the probes when the distances between them were 2.2, 3.2 and $4.2 \mathrm{~cm}$ for $\mathrm{C}-190$ and $\mathrm{S}_{\mathrm{r}}=0.5\left(\theta=0.170 \mathrm{~m}^{3} / \mathrm{m}^{3} \pm 1.98 \%\right.$ at $95 \%$ confidence level). It can be perceived that there is no interference since both waveforms are overlapping each other. The water contents determined by the two probes were 0.1737 and 0.1732 $\mathrm{m}^{3} / \mathrm{m}^{3}$, which are +2.18 and $+1.88 \%$ uncertainties comparing with the known $\theta$ of $0.170 \mathrm{~m}^{3} / \mathrm{m}^{3}$. However, when the distance was reduced to $1.0 \mathrm{~cm}$, some interference was observed as shown in Fig. 5.18. By looking at the waveforms in Fig. 5.18, it can be seen that the entire reflected waveform for probe 2 is slightly shifted to the right. Although the waveform was shifted, the apparent lengths of the two probes were similar; therefore, the determined water contents were 0.1753 and $0.1774 \mathrm{~m}^{3} / \mathrm{m}^{3}$, which are +3.12 and $+4.35 \%$ uncertainties. 


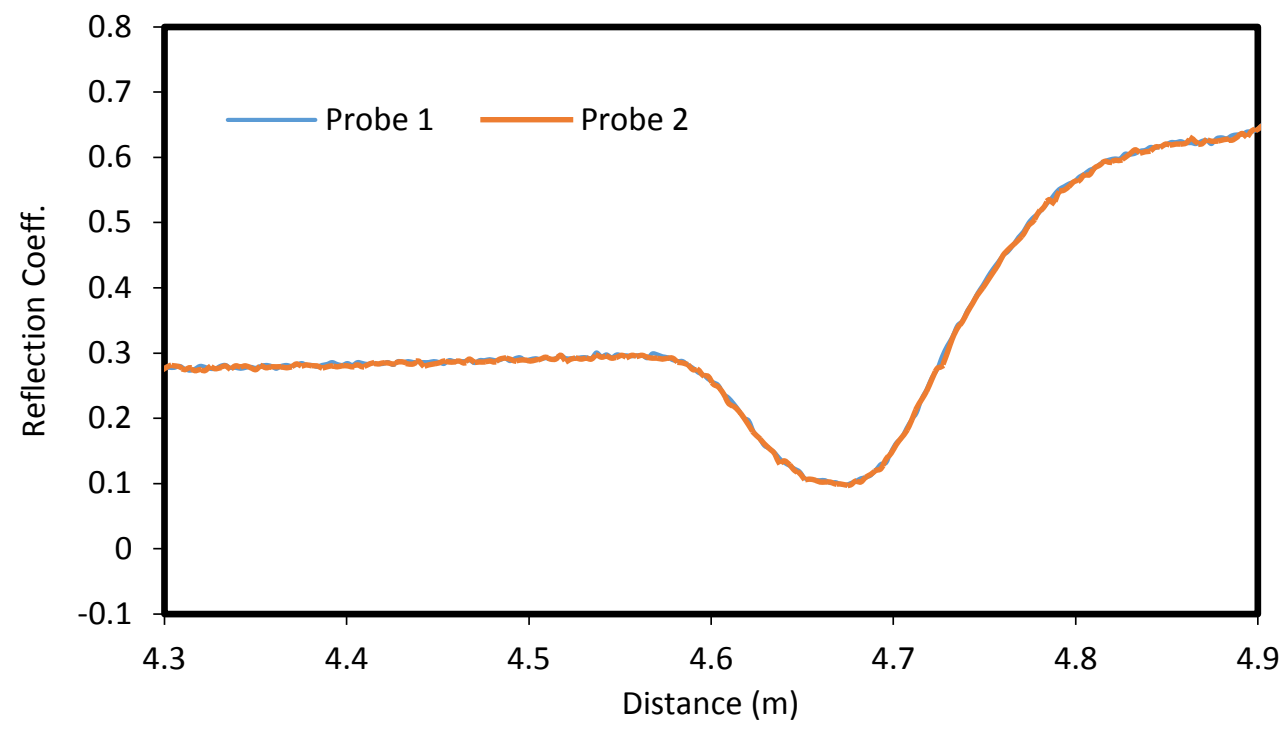

Fig. 5.17 The waveforms captured simultaneously by the probes placing 2.2, 3.2 and $4.2 \mathrm{~cm}$ apart in C-190 soil with $\mathrm{S}_{\mathrm{r}}=0.5$

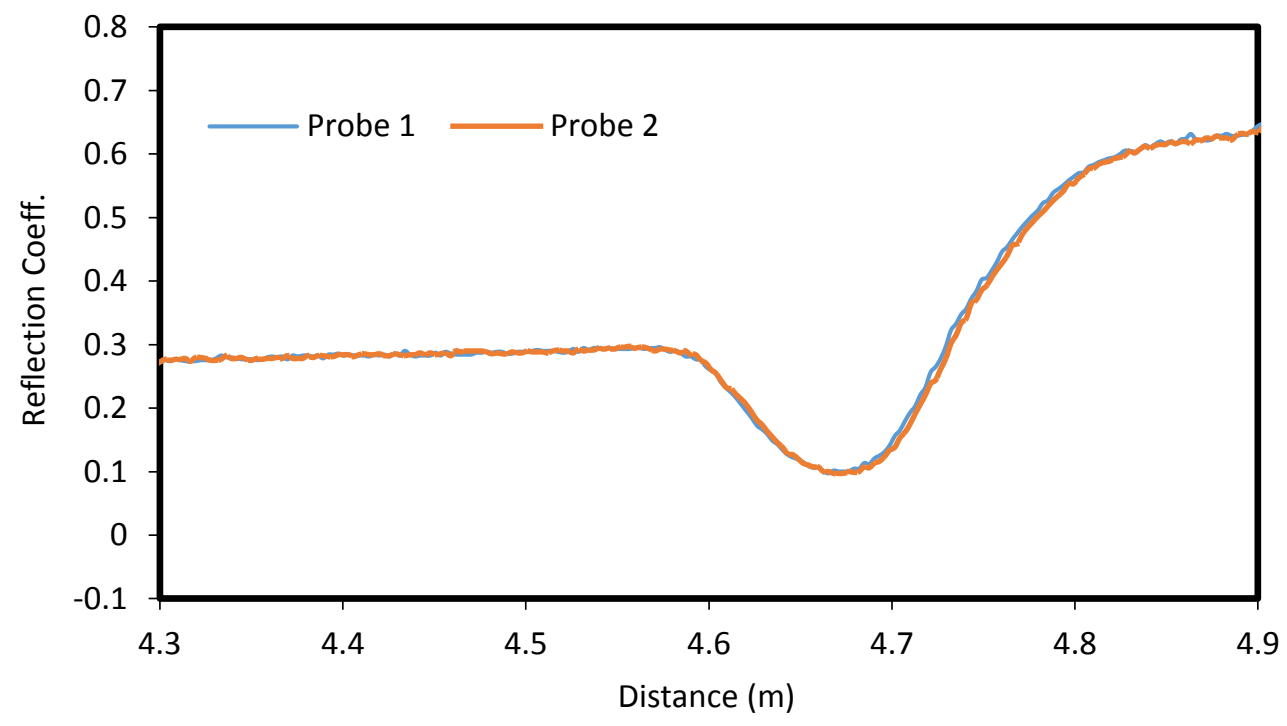

Fig. 5.18 The waveforms captured simultaneously by the probes placing $1.0 \mathrm{~cm}$ apart in C-190 soil with $\mathrm{S}_{\mathrm{r}}=0.5$ 
Fig. 5.19 illustrates the waveforms that were captured by the probes when the distances between them were 2.2, 3.2 and $4.2 \mathrm{~cm}$ for $\mathrm{C}-190$ and $\mathrm{S}_{\mathrm{r}}=1.0\left(\theta=0.340 \mathrm{~m}^{3} / \mathrm{m}^{3} \pm 1.61 \%\right.$ at $95 \%$ confidence level). It can be perceived that there is again no interference since both waveforms are overlapping each other. The water contents determined by the two probes were 0.3434 and 0.3452 $\mathrm{m}^{3} / \mathrm{m}^{3}$, which are +1.00 and $+1.53 \%$ uncertainties comparing with the known $\theta$ of $0.340 \mathrm{~m}^{3} / \mathrm{m}^{3}$. However, when the distance was reduced to $1.0 \mathrm{~cm}$, some interference was observed as shown in Fig. 5.20. By looking at the waveforms in Fig. 5.20, it can be seen that, similar to ON-3, the latter half of the reflected waveform for probe 2 is slightly shifted downward and to the right. This uneven shift caused the apparent lengths of the two probes to be dissimilar; therefore, the determined water contents were 0.3478 and $0.3499 \mathrm{~m}^{3} / \mathrm{m}^{3}$, which are +2.29 and $+2.91 \%$ uncertainties.

Comparing the waveforms shown in Figs. 5.15, 5.17 and 5.19, it can be realized that increase of water content in C-190 would induce no interference effect, when the distances between probes were 2.2,3.2 and $4.2 \mathrm{~cm}$; the analysis of these waveforms results in the same water content values for the two probes. The increase of water content would change the features of the waveform. That is, as the water content increases, the reflected waveform would be deeper and wider, resulting in longer apparent length of the probe or higher dielectric constant of the soil.

Figs. 5.16, 5.18 and 5.20 illustrate the waveforms that were captured by the probes when the distance between them was $1.0 \mathrm{~cm}$ for C-190. It can be seen that, unlike ON-3, the severity of interference does not increases as the water content increases. Up to $S_{r}=0.5$, the interference only causes horizontal shift of the waveforms without altering the results. However, at fully saturated condition $\left(S_{r}=1\right)$, the interference affects features of the waveform. By comparing with the previous observations, it can be concluded that both water content and distance between the probes influence the interference effect. It was determined that, similar to BC-1, for C-190 no interference occur, as a result of water content increase. However, when distance between probes was reduced to $1.0 \mathrm{~cm}$, as shown in Figs. 5.16, 5.18 and 5.20, the accuracy of TDR measurements was slightly affected by the interference but uncertainties were well within 5\% and not increased as a result of water content increase. It can be concluded that for coarse soil (sand) the effect of interference is negligible even when the water content is high and the distance between probes is short. 


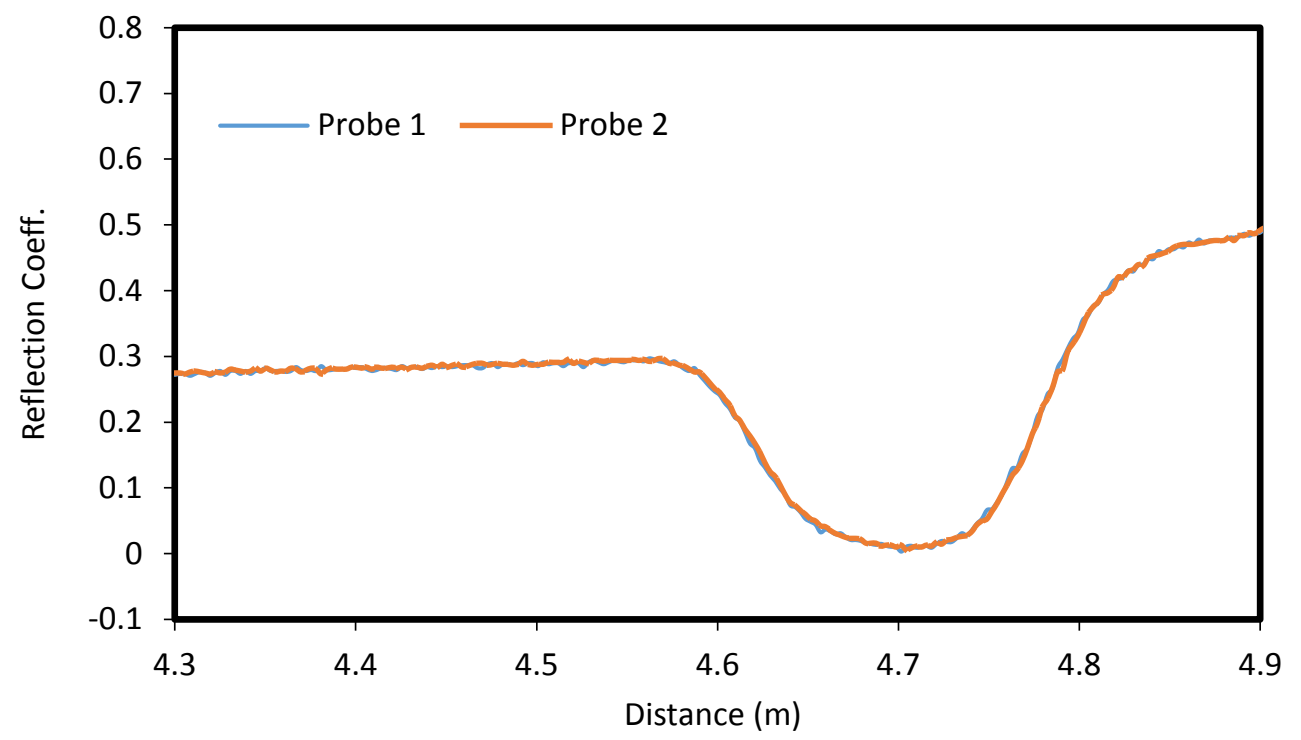

Fig. 5.19 The waveforms captured simultaneously by the probes placing 2.2, 3.2 and $4.2 \mathrm{~cm}$ apart in C-190 soil with $S_{\mathrm{r}}=1$

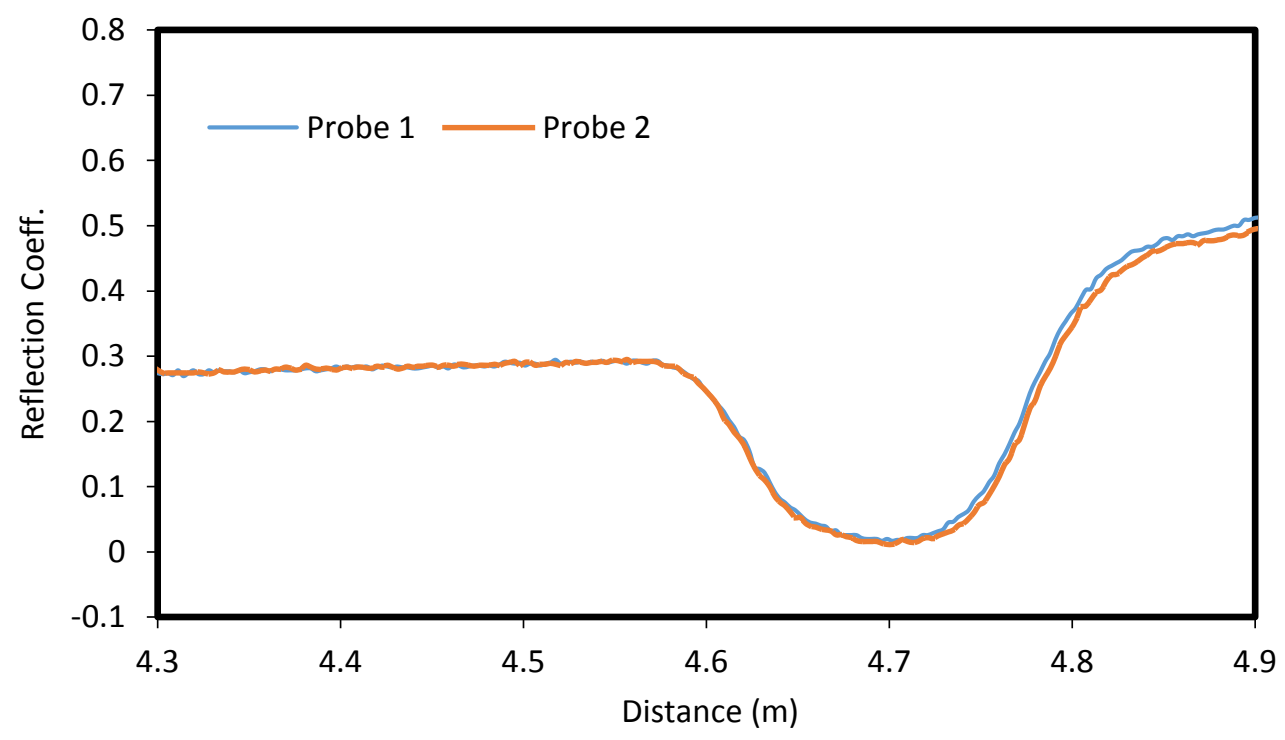

Fig. 5.20 The waveforms captured simultaneously by the probes placing $1.0 \mathrm{~cm}$ apart in C-190 soil with $S_{\mathrm{r}}=1$

The results are summarized in Table 5.1. In general, it can be concluded that the effect of interference of two adjacent TDP probes only becomes significant when the TDR probes are very close to about $1 \mathrm{~cm}$ apart. For all three types of soils the probes were also inserted within several 
different distances from each other within the range of $12 \mathrm{~mm}$ to $22 \mathrm{~mm}$ and no difference in measurements were observed. The effect of interference is also highly depends on the soil texture; a fine soil (such as, silty clay in this study) is highly susceptible to interference. Even for low water content $\left(S_{\mathrm{r}}=0.25\right)$ the effect of interference can cause about $10 \%$ uncertainty in TDR measurements of water content; as the water content increases, the uncertainty also increases up to about $15 \%$ when the soil is fully saturated $\left(S_{\mathrm{r}}=1\right)$. For the other two soils, which are medium coarse (loamy sand) and coarse (sand), the effect of interference is not significant and only causes measurement uncertainties of less than 5\%, which is the overall uncertainty of sample preparation at $95 \%$ confidence level.

\subsection{Results Summary}

The interference effect between two proximate TDR probes was examined. It was observed that the distance between the probes, soil texture and the water content of the testing medium are the key parameters which affect the interference and TDR probe capability of measurement. In this study, for the specified probes, it was found that parallel TDR probes can interfere with each other if the distance between them is around $1 \mathrm{~cm}$. It was also found that stronger interference can be detected at higher water contents. In this study three types of soils were examined, namely FSJ\#1 (silty clay), Matilda (loamy sand) and Ottawa sand C-190 (sand). Three different volumetric water contents were evaluated for each soil, namely: $S_{r}=0.25,0.5$ and 1 . The interference effect was noticeable in all soil samples when the distances between the probes were $1 \mathrm{~cm}$. For the samples with higher water content, the effect of interference on the TDRs' electromagnetic waveform signals is more prominent. Interference was found in Matilda samples with degrees of saturations of 0.5 and higher even though the distances between the probes were greater than 1 $\mathrm{cm}$; nevertheless, the determination of volumetric water content was not affected by the interference, because the interference only caused the two TDRs' electromagnetic waveform signals to shift by the same length. No interference was observed in cases of FSJ\#1 and Ottawa sand when the distances between the probes were $2.2,3.2$ and $4.2 \mathrm{~cm}$. It can be concluded that soils with more clay content are more susceptible to interference and result in significant TDR measurement uncertainty of water content. For FSJ\#1 (silty clay) it was observed that, during the event of interference, increase in soil water content induces higher measurement uncertainty of the TDR probes up to about $15 \%$ at full saturation conditions. 
Table 5.1: Summary of the Results of TDR Interference

\begin{tabular}{|c|c|c|c|}
\hline Soil type & $\mathrm{S}_{\mathrm{r}}$ & $\begin{array}{c}\text { Distance between two probes } \\
2.2,3.2 \text { and } 4.2 \mathrm{~cm}\end{array}$ & $\begin{array}{c}\text { Distance between two probes } \\
1 \mathrm{~cm}\end{array}$ \\
\hline \multirow{3}{*}{$\begin{array}{c}\text { ON-3 (Matilda) } \\
3.6 \% \text { clay, } 25.5 \% \\
\text { Silt and } 70.9 \% \\
\text { Sand }\end{array}$} & 0.25 & $\begin{array}{l}\text { No interference. No effect on } \\
\text { TDR capability of } \\
\text { measurement. }\end{array}$ & $\begin{array}{l}\text { Interference effect exists. No } \\
\text { effect on TDR capability of } \\
\text { measurement (<5\% error). }\end{array}$ \\
\hline & 0.5 & $\begin{array}{l}\text { Very slight interference. No } \\
\text { effect on TDR capability of } \\
\text { measurement. }\end{array}$ & $\begin{array}{l}\text { Greater interference effect than } \\
\text { the case of } S_{\mathrm{r}}=0.25 \& 1 \mathrm{~cm} \text {. } \\
\text { Slight effect on TDR capability } \\
\text { of measurement }(<5 \% \text { error). }\end{array}$ \\
\hline & 1 & $\begin{array}{c}\text { Slight interference. No effect } \\
\text { on TDR capability of } \\
\text { measurement. }\end{array}$ & $\begin{array}{l}\text { Greater interference effect than } \\
\text { the case of } S_{\mathrm{r}}=0.5 \& 1 \mathrm{~cm} \text {. } \\
\text { Slight effect on TDR capability } \\
\text { of measurement }(<5 \% \text { error). }\end{array}$ \\
\hline \multirow{3}{*}{$\begin{array}{l}\text { BC-1 ( FSJ\#1) } \\
41.8 \% \text { clay and } \\
58.2 \% \text { Silt }\end{array}$} & 0.25 & $\begin{array}{c}\text { No Interference. No effect on } \\
\text { TDR capability of } \\
\text { measurement. }\end{array}$ & $\begin{array}{l}\text { Interference effect exists. Effects } \\
\text { TDR capability of measurement } \\
\text { ( } ~ 10 \% \text { error }) .\end{array}$ \\
\hline & 0.5 & $\begin{array}{c}\text { No Interference. No effect on } \\
\text { TDR capability of } \\
\text { measurement. }\end{array}$ & $\begin{array}{l}\text { Greater interference effect than } \\
\text { the case of } S_{\mathrm{r}}=0.25 \& 1 \mathrm{~cm} . \\
\text { Effects TDR capability of } \\
\text { measurement }(\sim 13 \% \text { error }) \text {. }\end{array}$ \\
\hline & 1 & $\begin{array}{c}\text { No Interference. No effect on } \\
\text { TDR capability of } \\
\text { measurement. }\end{array}$ & $\begin{array}{l}\text { Greater interference effect than } \\
\text { the case of } S_{\mathrm{r}}=0.5 \& 1 \mathrm{~cm} . \\
\text { Effects TDR capability of } \\
\text { measurement }(\sim 15 \% \text { error }) \text {. }\end{array}$ \\
\hline \multirow{3}{*}{$\begin{array}{l}\text { C-190 (Ottawa } \\
\text { Sand) } 100 \% \\
\text { Sand }\end{array}$} & 0.25 & $\begin{array}{c}\text { No Interference. No effect on } \\
\text { TDR capability of } \\
\text { measurement. }\end{array}$ & $\begin{array}{l}\text { Interference effect exists. Slight } \\
\text { effect on TDR capability of } \\
\text { measurement (<5\% error). }\end{array}$ \\
\hline & 0.5 & $\begin{array}{c}\text { No Interference. No effect on } \\
\text { TDR capability of } \\
\text { measurement. }\end{array}$ & $\begin{array}{l}\text { Greater interference effect than } \\
\text { the case of } S_{\mathrm{r}}=0.25 \& 1 \mathrm{~cm} \text {. } \\
\text { Slight effect on TDR capability } \\
\text { of measurement }(<5 \% \text { error). }\end{array}$ \\
\hline & 1 & $\begin{array}{c}\text { No Interference. No effect on } \\
\text { TDR capability of } \\
\text { measurement. }\end{array}$ & $\begin{array}{l}\text { Greater interference effect than } \\
\text { the case of } S_{\mathrm{r}}=0.5 \& 1 \mathrm{~cm} \text {. } \\
\text { Slight effect on TDR capability } \\
\text { of measurement }(<5 \% \text { error). }\end{array}$ \\
\hline
\end{tabular}




\section{CHAPTER 6}

\section{EXPERIMENTAL RESULTS OF HEAT AND MASS TRANSFER IN A SOIL}

\subsection{Introduction}

A study of one-dimensional heat and moisture transfer within a vertical soil column was conducted experimentally. An experimental soil cell made of stainless-steel tube was exposed to differential heating by sandwiching it between two differentially-heated plates for studying heat and moisture transfer in the soil column at different temperature levels and temperature differences. The main objective of the experimental study was to investigate heat and moisture transfer characteristics in a soil at temperatures greater than $40^{\circ} \mathrm{C}$ up to $90^{\circ} \mathrm{C}$. In this chapter, the results are divided into two parts. In the first part, the results of heat transfer in dry soil are presented and discussed. The main objective was to investigate temperature distributions and heat gains/losses at the steady-state conditions along the soil cell at the various temperature levels. From the experiments it was observed that the steady-state conditions can be reached after about 4.5 hours for the cases of dry soil. The study of dry soil was beneficial because the capability of the apparatus to have one-dimensional heat transfer could be examined in the most critical condition. The critical condition was expected for dry soil because of its low thermal conductivity; therefore, the highest temperature and heat transfer deviations were expected to occur within the soil. In the second part, the results of heat and moisture transfer in wet soil are presented and discussed. In this part, the thermal properties and moisture contents were obtained within the time period of 2.5 hours for each test using the heat pulse technique. From the experiments, it was observed that the steady-state conditions can be reached after about 2 hours for the cases of wet soil. The wet sample of Matilda soil (ON-3) was prepared to have a saturation degree of 0.65 and a porosity of 0.40 . The tests were carried out using the modified model \#3. The engineering CAD drawing of the modified model \#3 is presented in Appendix B.5. After a soil sample was prepared and packed into the stainless-steel soil cell, the five T-TDR probes were inserted into the soil cell from the side along the axial direction of the soil cell at an equal distance of $29.6 \mathrm{~mm}$ between the centers of the probes. In total, twelve temperature measurements were recorded every second along $147.9 \mathrm{~mm}$ height of the soil column. 


\subsection{Heat Transfer in Dry Soil}

A number of tests were carried out to investigate the heat transfer in dry soil. For the series of tests, dry soil was evenly packed in the stainless-steel cylindrical tube and was vertically sandwiched between the hot and cold plates. The two heat flux meters (HFMs) were placed separately at both ends of the soil column to measure the heat fluxes entering and exiting the soil, as well as to measure the temperatures of the HFMs at the top and bottom of the soil column. The HFMs contain type-T thermocouples embedded in them. The tests were carried out at various temperature levels while the temperature difference between the top and bottom plates was kept at about $15^{\circ} \mathrm{C}$. The temperature level is defined as the average temperature between the temperatures of the HFMs, and the temperature difference is defined as the temperature difference between the temperatures of the HFMs. In all cases, when plotting the temperature distributions along the soil column in the subsequent figures, the linear line connecting the temperatures of the HFMs was considered to be a reference temperature distribution along the soil column for a one-dimensional heat transfer condition.

\subsubsection{Observations and Analysis of Results}

Figs. 6.1 to 6.4 illustrate temperature variations along the soil column for the modified model \#3 at four different temperature levels of 37.9, 52.9, 67.8 and $82.6^{\circ} \mathrm{C}$, respectively, at steady state conditions. Since all four temperature levels were higher than the ambient temperature of about $23^{\circ} \mathrm{C}$, there was heat loss from the entire soil cell to the ambient air, causing the entire measured soil temperature profiles to be below the reference linear temperature profile with the highest deviations occurred at the top part of the soil column. From the results, it was found that the maximum temperature deviation from the linear temperature profile occur at around $40 \mathrm{~mm}$ from the top of the soil column. It can be seen from Figs. 6.1 to 6.4 that the deviation increases as the temperature level increases. 


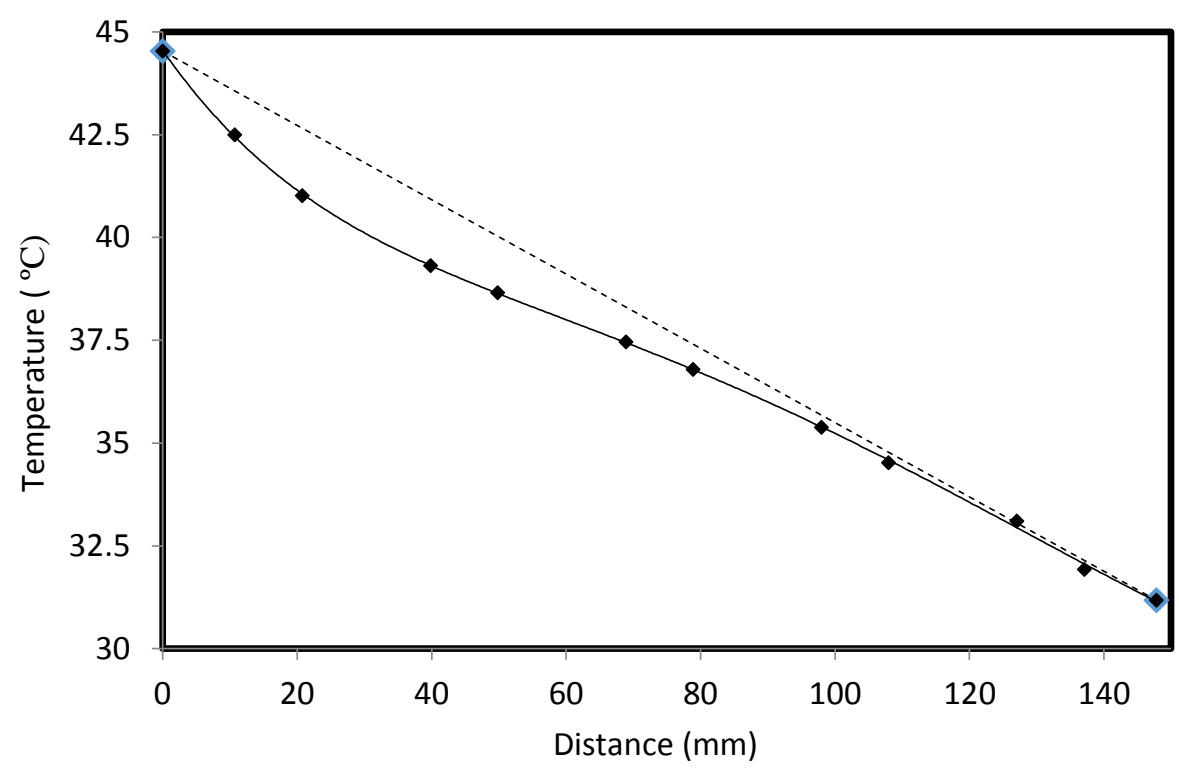

Fig. 6.1 Temperature variations along dry soil column from the top, where the distance is $0 \mathrm{~mm}$, to the bottom, where the distance is $147.9 \mathrm{~mm}$ at the temperature level of $37.9^{\circ} \mathrm{C}$ and the temperature difference of $13.4^{\circ} \mathrm{C}$

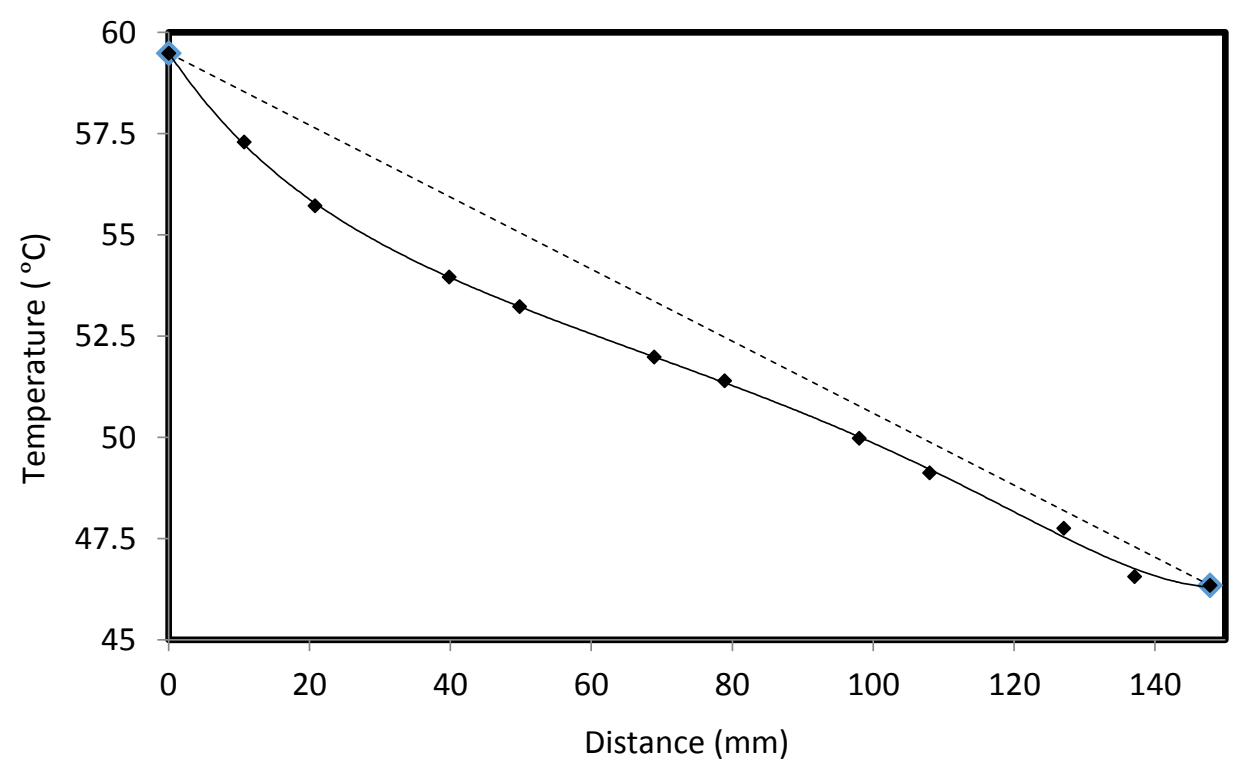

Fig. 6.2 Temperature variations along dry soil column from the top, where the distance is $0 \mathrm{~mm}$, to the bottom, where the distance is $147.9 \mathrm{~mm}$ at the temperature level of $52.9^{\circ} \mathrm{C}$ and the temperature difference of $13.1^{\circ} \mathrm{C}$ 


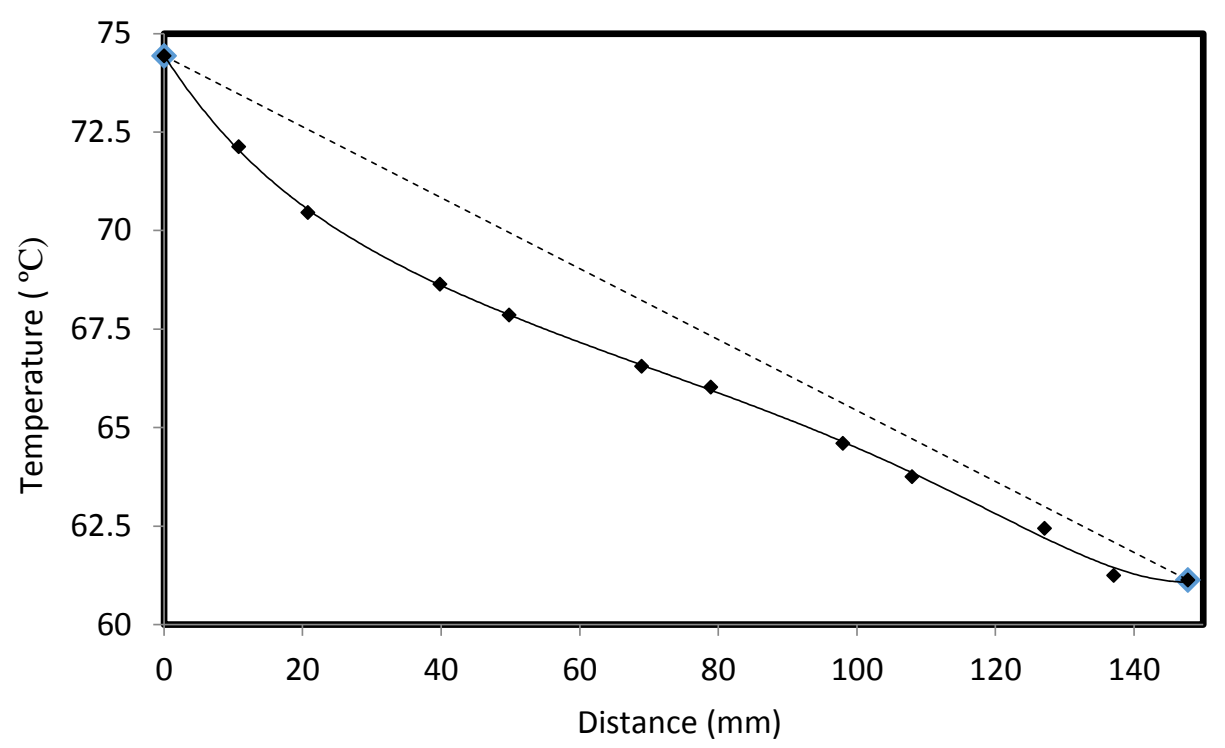

Fig. 6.3 Temperature variations along dry soil column from the top, where the distance is $0 \mathrm{~mm}$, to the bottom, where the distance is $147.9 \mathrm{~mm}$ at the temperature level of $67.8^{\circ} \mathrm{C}$ and the temperature difference of $13.3^{\circ} \mathrm{C}$

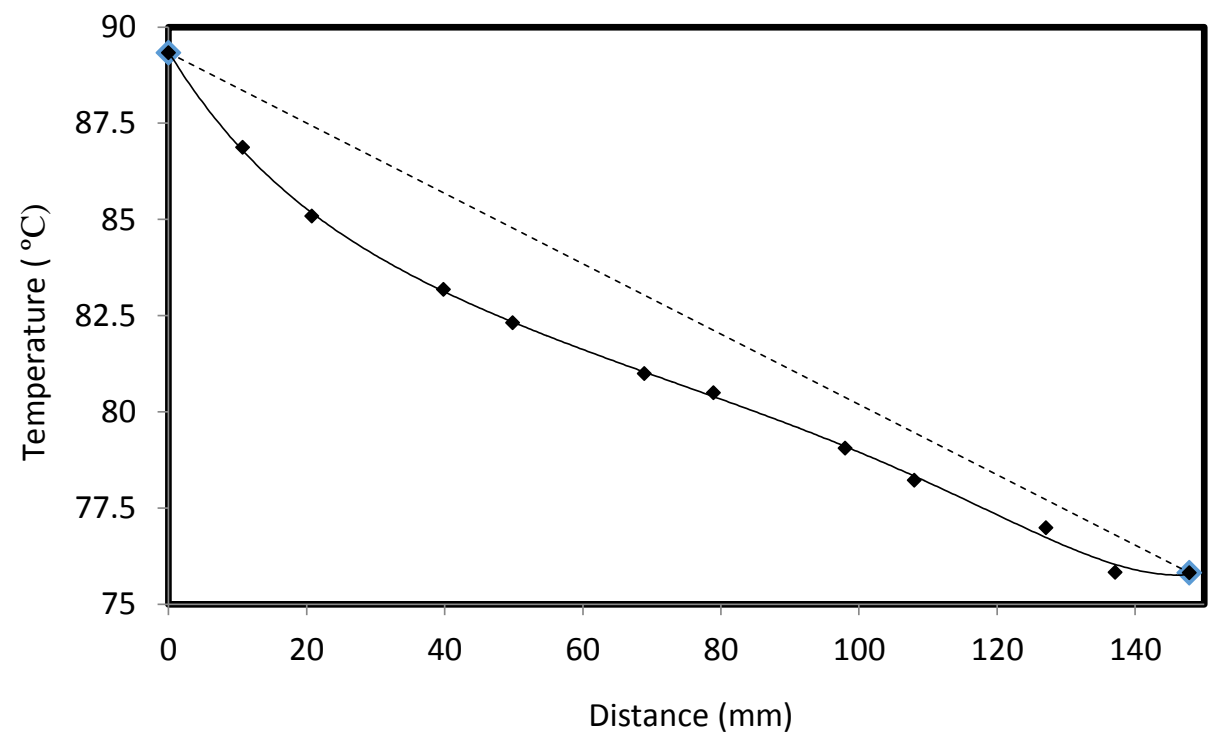

Fig. 6.4 Temperature variations along dry soil column from the top, where the distance is $0 \mathrm{~mm}$, to the bottom, where the distance is $147.9 \mathrm{~mm}$ at the temperature level of $82.6^{\circ} \mathrm{C}$ and the temperature difference of $13.5^{\circ} \mathrm{C}$ 
Fig. 6.5 presents the highest percent deviations from the linear temperature profile (LTP) with respect to the overall temperature difference, $\Delta T=T_{H F M, t o p}-T_{H F M, \text { bottom }}$, at different temperature levels.

$\%$ Temperature Deviation $=\frac{\left(T_{L T P, x}-T_{x}\right)_{\max }}{\Delta T} \times 100$

where $T_{L T P, x}$ is the temperature of the LTP at a distance $x$ from the top of the soil column, and $T_{x}$ is the measured soil temperature at the same distance $x$. Fig. 6.5 also illustrates the differences of heat fluxes, measured by the heat flux meters.

$\%$ Difference of Heat Flux $=\frac{q_{H F M, \text { top }}-q_{H F M, \text { bottom }}}{q_{H F M, \text { bottom }}} \times 100$

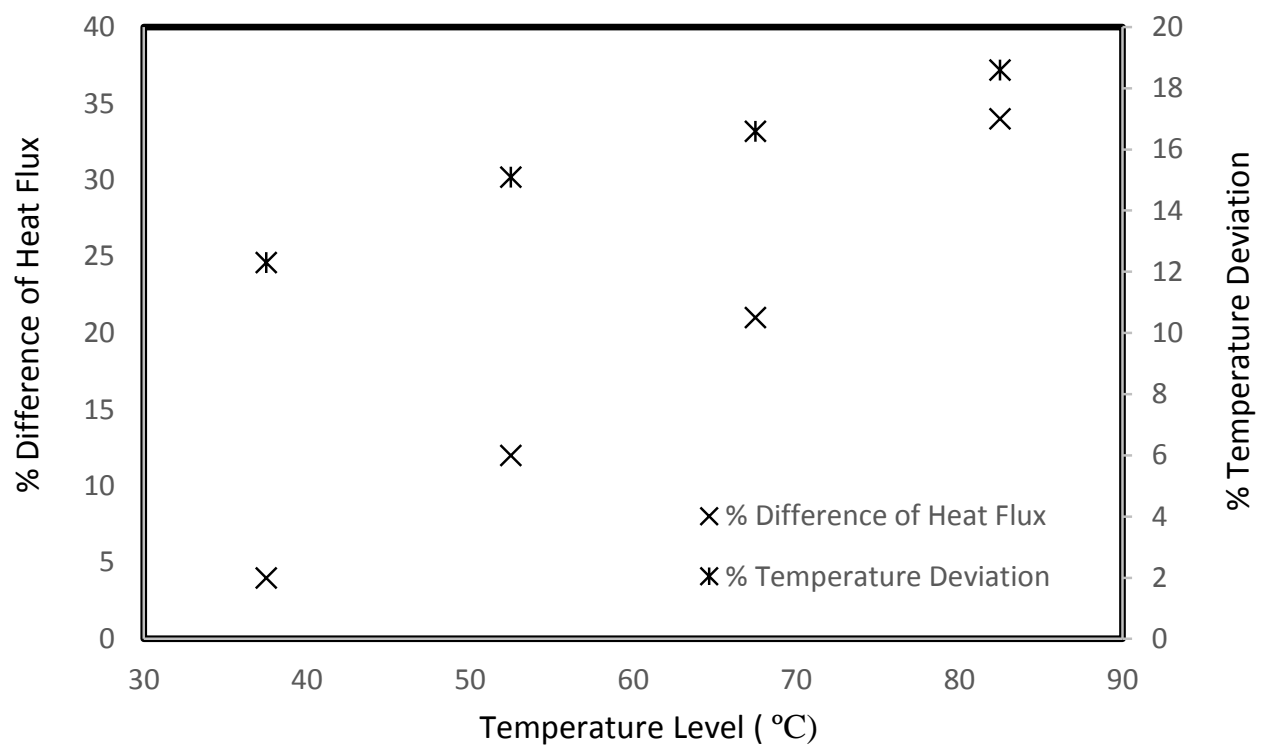

Fig. 6.5 The results of highest percent temperature deviations from the linear temperature distribution with respect to overall temperature differences and the percent difference of heat fluxes between the inlet and the outlet of dry soil cell at four different temperature levels

where $q_{H F M, t o p}$ and $q_{H F M, b o t t o m}$ are the heat fluxes measured by the top and bottom HFMs, respectively. It was found that the highest temperature deviation is $18.6 \%$ at the temperature level of $82.6^{\circ} \mathrm{C}$ and the temperature gradient of about $90^{\circ} \mathrm{C} / \mathrm{m}$. This deviation is an indication of the existence of two-dimensional heat transfer in the soil column, as some of the heat was conducted 
radially toward the stainless-steel tube wall. This phenomenon is particularly noticeable due to the low thermal conductivity of dry soil $\left(k_{d r y}\right)$ relative to the thermal conductivity of stainless steel tube wall $\left(k_{s s}\right)$, i.e. $k_{d r y} / k_{s s} \approx 0.015$. Thus, it can be realized that the heat conduction in the soil would rather bypass the soil and thermally short-circuited to the wall. It may seem that the temperature deviation due to heat loss is quite high; but, comparing to the amount of heat conduction through the soil column at the same temperature level of $82.6^{\circ} \mathrm{C}$ (in the order of 20 $\mathrm{W} / \mathrm{m}^{2}$ or $0.064 \mathrm{~W}$ ), the heat loss from the soil is in fact very small as indicated in Fig. 6.5 to be $34 \%$ difference between the top and bottom heat fluxes (i.e., in the order of $0.022 \mathrm{~W}$ ).

From the Fig. 6.4, it can be observed that there was higher heat losses in the upper portion of the soil cell due to the higher temperature difference between the upper portion temperature and the room temperature. In fact, since the heat conductivity of stainless steel is significantly larger than that of the dry soil and its surrounding insulation layer, the heat is forced to flow through the side walls. As the heat skip through the side walls the potential of soil to let the heat flow back from the side walls increases downstream simultaneously.

\subsection{Heat and Moisture Transfer in Wet Soil}

After the modified model \#3 was made, a series of tests were carried out for a wet soil (Matilda with $\mathrm{S}_{\mathrm{r}}=0.65$ and $\theta=0.26 \mathrm{~m}^{3} / \mathrm{m}^{3}$ ) to study heat and moisture transfer in soil. In this section, the focus of result presentation will be based on the most critical case which is the case of $90-10^{\circ} \mathrm{C}$, i.e. the hot and cold plates were set at 90 and $10^{\circ} \mathrm{C}$, respectively, or temperature level of $54.8^{\circ} \mathrm{C}$. This case was selected since the main purpose of this work is to study high temperature heat and moisture transfer in soil. The discussion of the results will be presented in two parts. In the first part, a general analysis of the results will be discussed, and in the second part more indepth analysis will be discussed. The results of the remaining cases, i.e. various temperature levels and temperature differences, are presented in graphical form in Appendix D.

\subsubsection{General Discussion of Results}

Fig. 6.6 illustrates the results of the highest percent temperature deviations from the linear temperature profile of four cases of temperature levels $\left(38.1^{\circ} \mathrm{C}, 52.9^{\circ} \mathrm{C}, 67.6^{\circ} \mathrm{C}\right.$ and $\left.82.1^{\circ} \mathrm{C}\right)$ with 
respect to the overall temperature differences $\left(12.6^{\circ} \mathrm{C}\right)$ and the percent differences of heat fluxes measured by the heat flux meters. It can be seen that the highest difference of heat fluxes would not be exceeding $4.2 \%$ and the highest temperature deviation in the worst case is not more than 7.7\%. This was expected, as compared with the results of the dry soil, because the wet soil has much higher thermal conductivity $(1.4$ vs. $0.26 \mathrm{~W} / \mathrm{m} \cdot \mathrm{K})$ which resulted in higher heat transfer through the soil. As a consequence, the amount of heat loss with respect to the heat transfer through the soil was relatively small; therefore, greater uniformity of heat flux in the radial direction would be achieved along the soil column, i.e. achieving one-dimensional heat and moisture transfer and resulting in closer linear temperature profile along the soil column. From Fig. 6.6 it was observed that the higher discrepancies of the results occur at higher temperature levels while the temperature differences remain relatively constant.

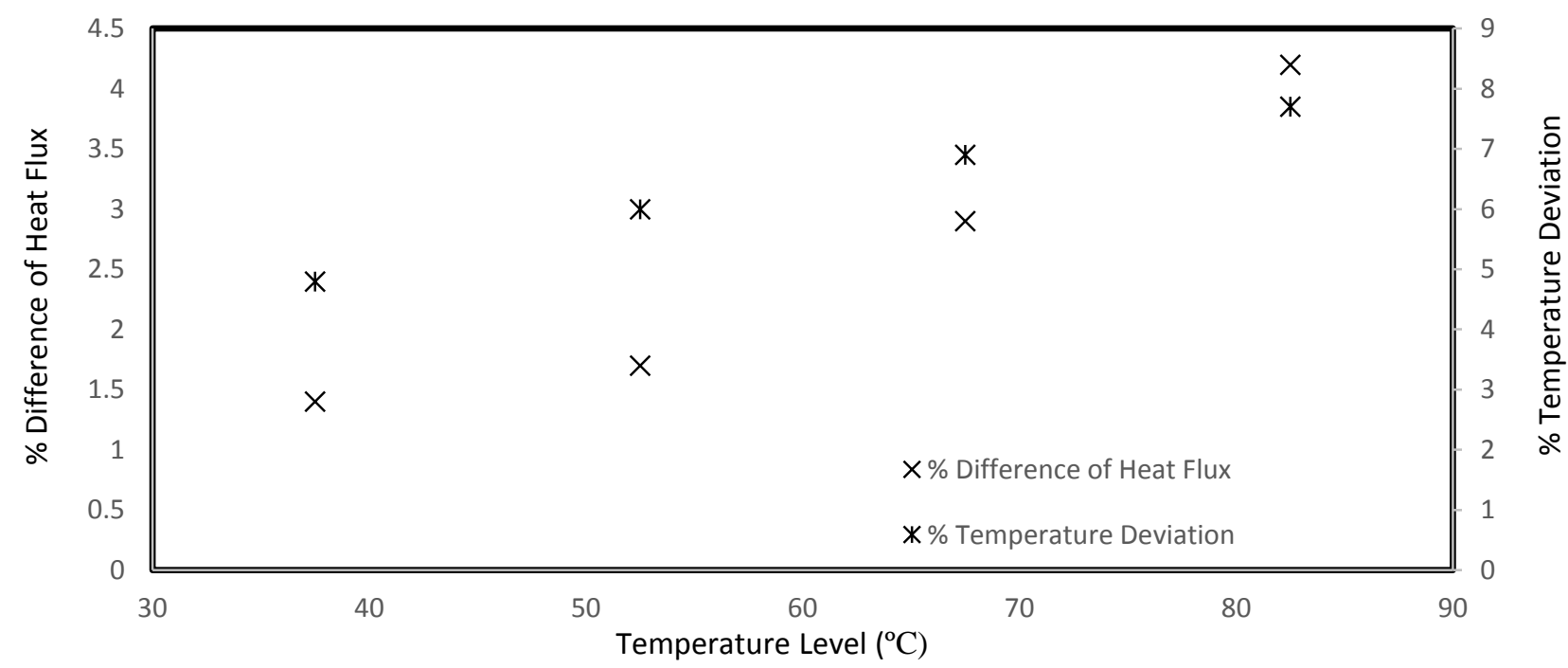

Fig. 6.6 The results of the highest percent temperature deviations from the linear temperature profile with respect to overall temperature differences and the percent differences of heat fluxes at four different temperature levels

Fig. 6.7 illustrates the results of the highest percent temperature deviations from the linear temperature profile with respect to overall temperature differences and the percent difference of heat fluxes at various temperature levels and temperature differences. It can be seen that although the highest deviation increases at higher temperature differences the percent difference of heat fluxes actually remains around 1\%. By carefully looking at the results it can be noticed that the percent difference of heat fluxes slightly reduces from $1.5 \%$ to $0.2 \%$ as temperature difference 
increases from about $17^{\circ} \mathrm{C}\left(50-30^{\circ} \mathrm{C}\right)$ to about $49^{\circ} \mathrm{C}\left(70-10^{\circ} \mathrm{C}\right)$. This is the range that the temperature level would not significantly increases (from 40 to $41.6{ }^{\circ} \mathrm{C}$ ). Later, the percent difference of heat fluxes slightly increases to $1 \%$ as temperature difference increases to roughly $57^{\circ} \mathrm{C}\left(80-10^{\circ} \mathrm{C}\right)$. However, this time the change in temperature level would be more significant (from 41.6 to $49.1^{\circ} \mathrm{C}$ ). This could increase heat loss from the soil cell due to higher temperature difference between the soil cell and the ambient air. Therefore, from the results, it can be concluded that generally speaking the percent difference of heat fluxes increases as temperature level increases and the percent difference of heat fluxes decreases as temperature difference increases.

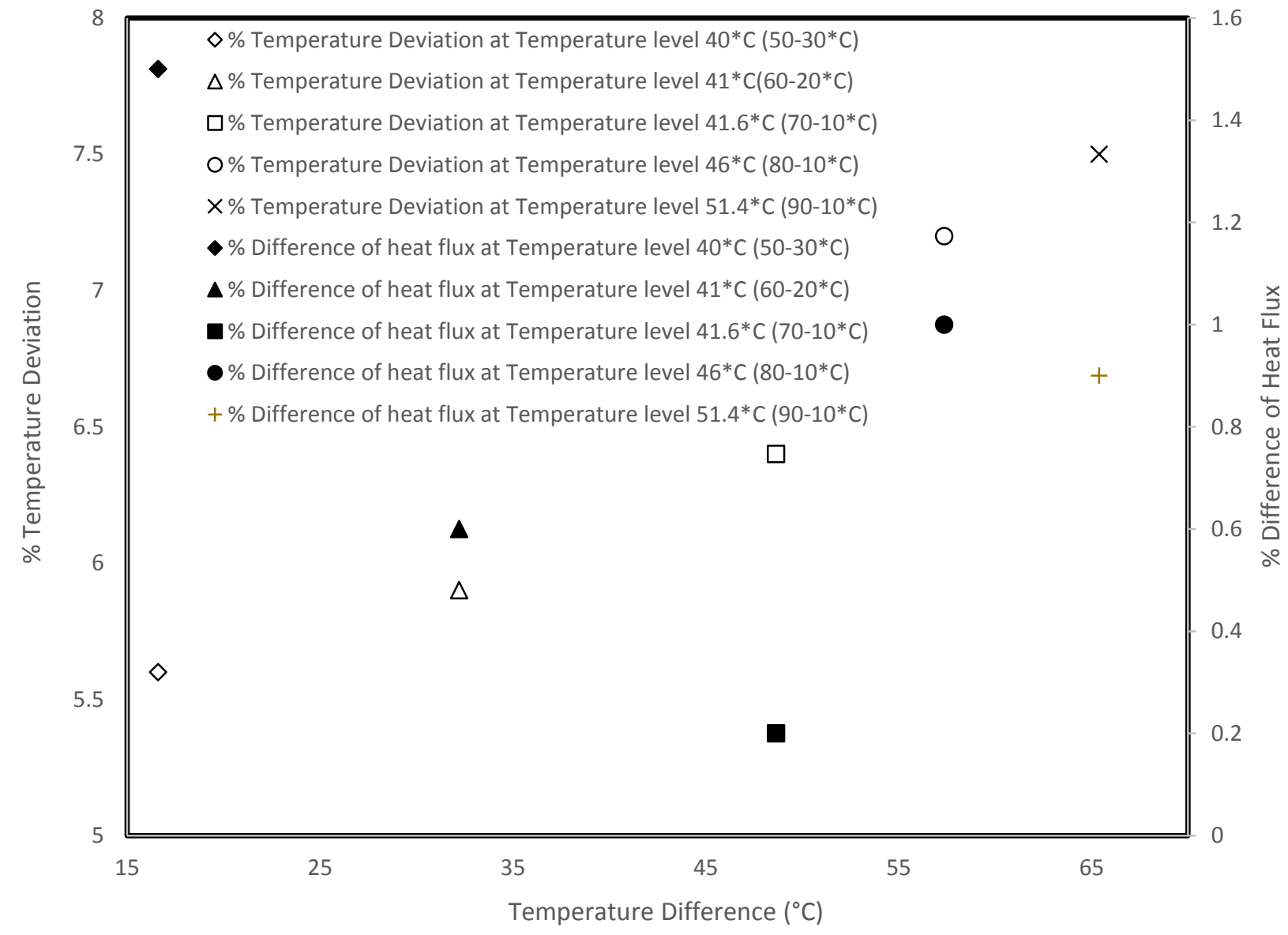

Fig. 6.7 The results of the highest percent temperature deviations from the linear temperature profile with respect to overall temperature differences and the percent differences of heat fluxes at various temperature levels and temperature differences

Suppose that the total volume of the soil cell was divided into five equal control volumes each associated with a T-TDR probe which was positioned in the center of the corresponding 
control volume. Fig. 6.8 shows schematic view of the soil cell's control volumes. Thus, the data (such as, temperature, volumetric water content, and soil properties obtained from each probe were reasonably assumed to represent the average values of the corresponding volume. Fig. 6.9 illustrates a general trend of temperature variations versus time along the soil column for the case of $90-10^{\circ} \mathrm{C}$. From the results it can be observed that the steady state condition was obtained after about 120 minutes into the test. It is interesting to see that although the temperature set point at the cold plate was set to be $10^{\circ} \mathrm{C}$, the temperature readings of the heat flux meter were highly affected by relatively large heat flux coming from the hot plate toward the bottom of the soil cell. The temperature gradient was increased to $442.4^{\circ} \mathrm{C} / \mathrm{m}$ at the steady state conditions. Due to large initial temperature difference (about $65^{\circ} \mathrm{C}$ ) between the hot plate and the top part of the soil, the temperature rise at the points closer to the upper hot plate is more rapid than the temperature drop at the points closer to the lower cold plate, which has only about $11^{\circ} \mathrm{C}$ difference between the cold plate and the bottom part of the soil. By carefully looking at the results, it can be noticed that until 30 minutes past into the test the lower region of the soil column was not yet significantly affected by the heat flux from the upper side. Therefore, under the effect of the cold plate, the temperature at the bottom of soil column remained at about $13.6^{\circ} \mathrm{C}$; not until at some point in time (30 to 60 minutes), the temperature at the bottom soil column began to rise up to about $17.3^{\circ} \mathrm{C}$. This indicates that the heat would take about an hour to reach the bottom of the soil column for a temperature difference of $65.4^{\circ} \mathrm{C}$. Based on the temperature gradient at the bottom of the soil column at 15 minutes into the test and the measured soil thermal conductivity of $1.4 \mathrm{~W} / \mathrm{m} \cdot \mathrm{K}$, the heat flux was found to be about $463 \mathrm{~W} / \mathrm{m}^{2}$ from the soil to the cold plate. Therefore, the thermal resistance between the heat flux meter and the cold plate was estimated to be $0.0078 \mathrm{~m}^{2} \cdot \mathrm{K} / \mathrm{W}$. When the heat from the hot plate was finally transferred to the bottom of the soil column between 30 to 60 minutes into the test, the heat flux increased up to $1468 \mathrm{~W} / \mathrm{m}^{2}$, which resulted in the temperature rise of the heat flux meter by about $11.5^{\circ} \mathrm{C}$ due to the thermal resistance, in order to transfer the high heat flux into the cold plate. 


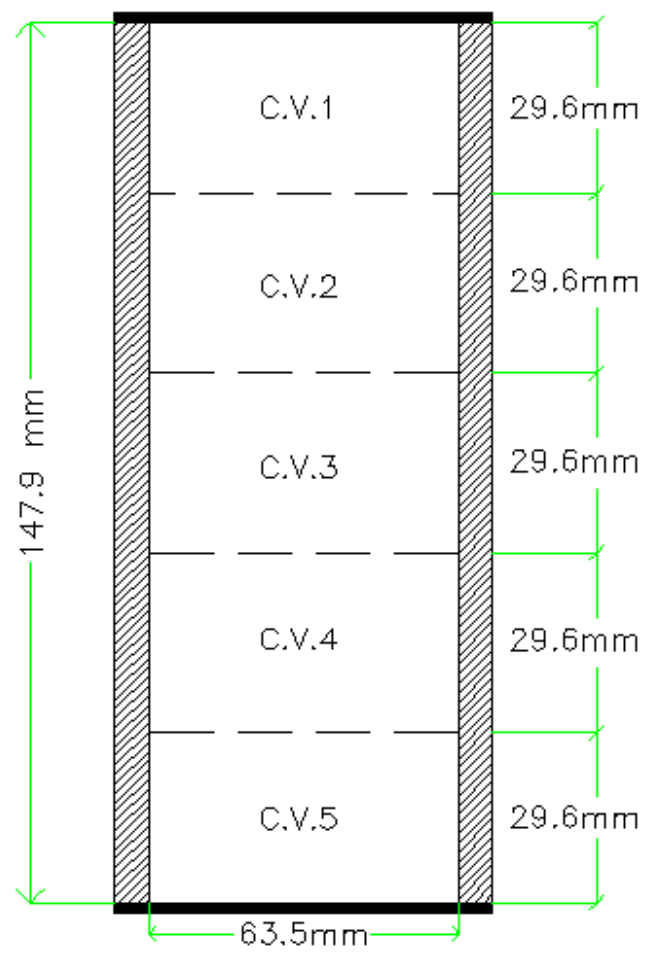

Fig. 6.8 Schematic view of soil cell's control volumes

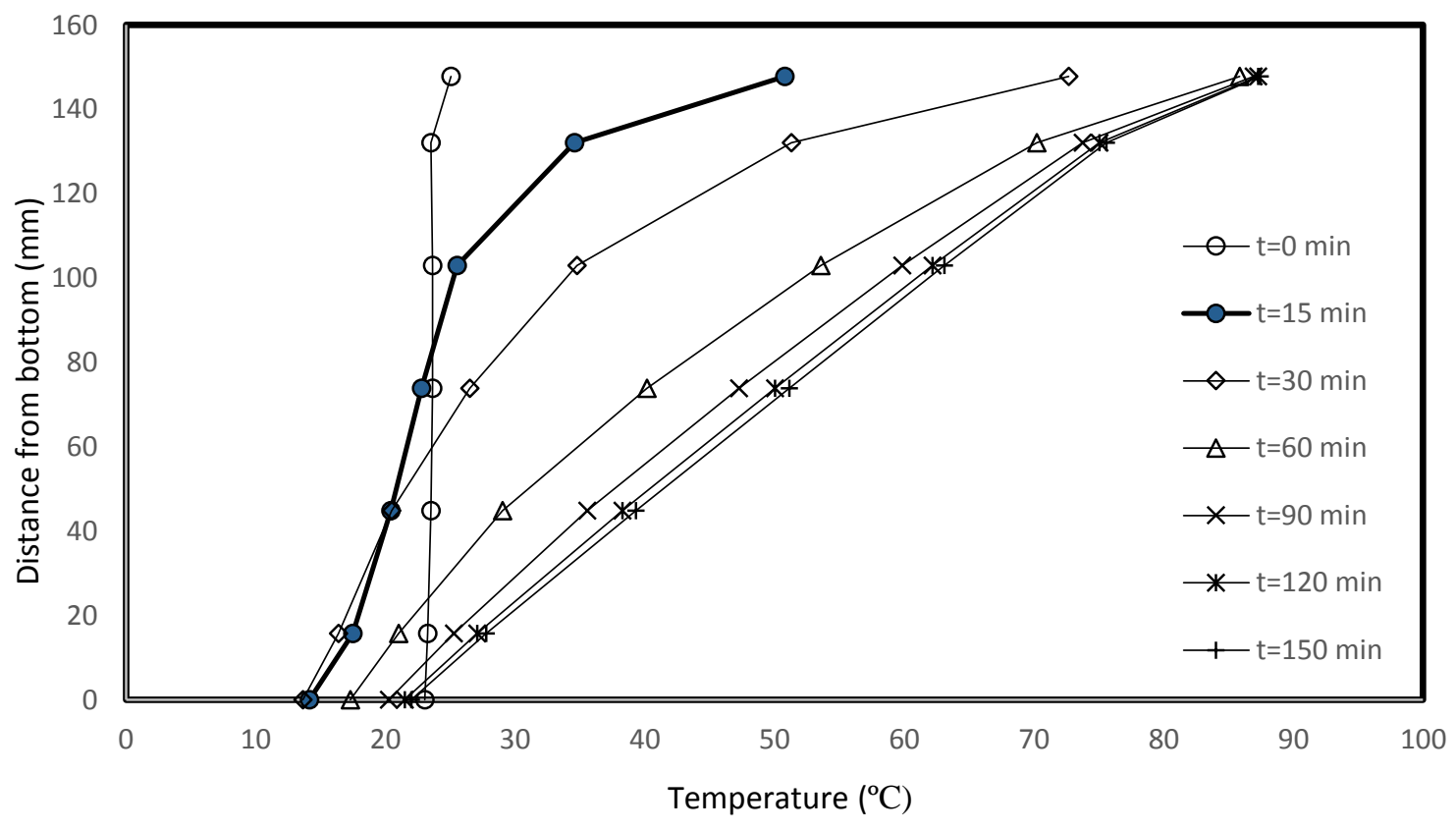

Fig. 6.9 Variations of the temperature vs. time along the soil column 
Fig. 6.10 illustrates a general trend of degree of saturation variation versus time along the soil column for the case of $90-10^{\circ} \mathrm{C}$. From the results, it can be observed that the moisture contents in the two upper control volumes (control volumes 1 and 2) dramatically dropped over time; while in the two lowest control volumes (control volumes 4 and 5), their moisture contents correspondingly increased. By comparing the results of moisture variations with the results of temperature variations, it can be understood that the significant increase of the temperature gradients in control volumes 1 and 2 was the key factor which induced the rapid moisture transfer out from the control volumes. The liquid water flowing down the soil column would be collected in the lower control volumes. Eventually, after about 2 hours, control volumes 3 to 5 are fully saturated, and control volumes 1 and 2 became almost completely dry.

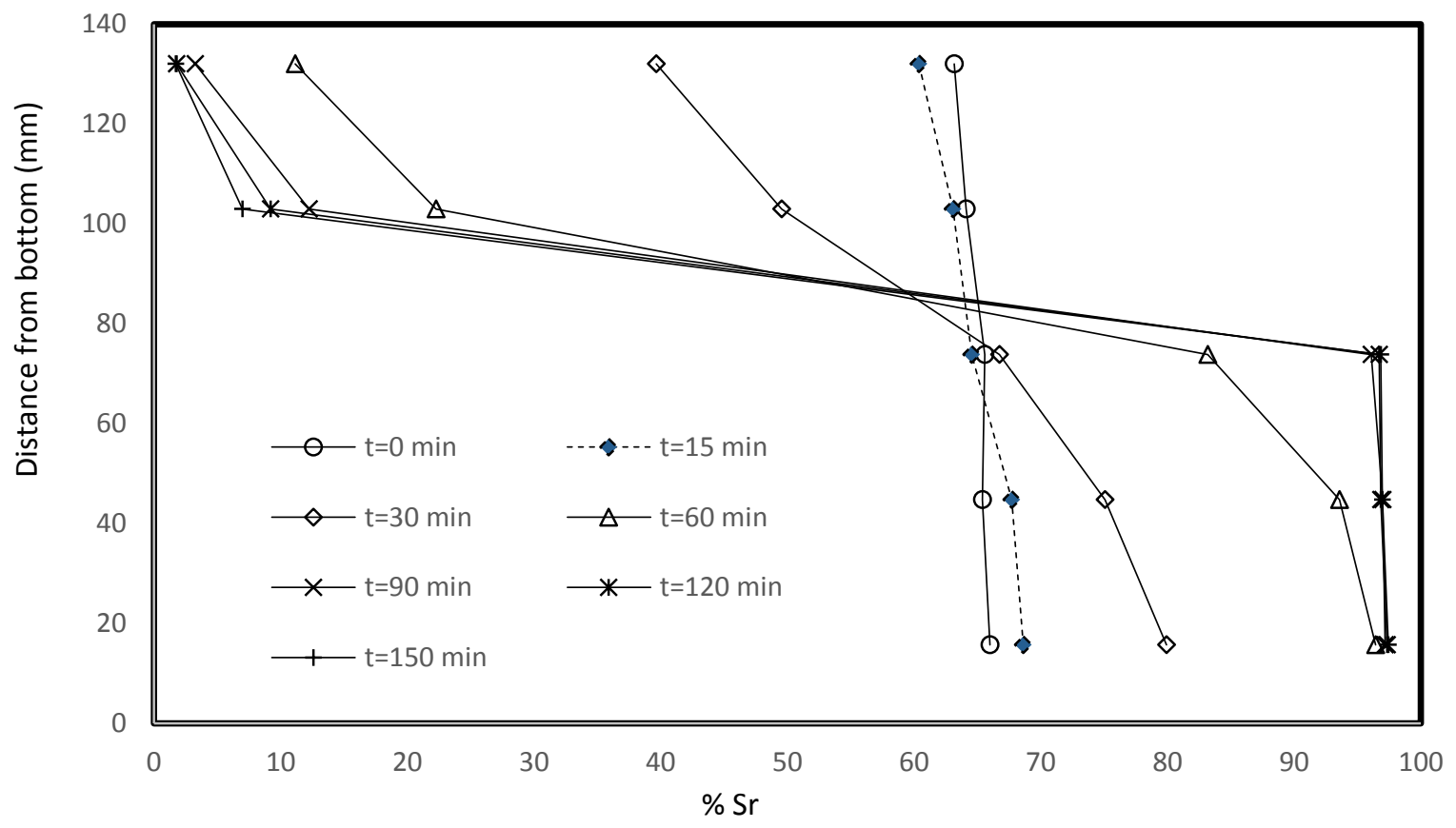

Fig. 6.10 Variations of the degree of saturation vs. time along the soil column 
Fig. 6.11 illustrates variations of the thermal properties vs. time along the soil column. From the results it can be perceived that all the thermal properties such as volumetric heat capacity $\left(\mathrm{J} / \mathrm{m}^{3} \cdot \mathrm{K}\right)\left(\right.$ Fig. 6.11(a)), thermal conductivity $(\mathrm{W} / \mathrm{m} \cdot \mathrm{K})\left(\right.$ Fig. 6.11(b)) and thermal diffusivity $\left(\mathrm{m}^{2} / \mathrm{s}\right)$ (Fig. 6.11(c)) are mainly affected by the moisture content variations. Volumetric heat capacity and thermal conductivity increase as moisture content increases; however, thermal diffusivity decreases as moisture content increases. Comparing the results of moisture variations versus time (Fig. 6.10) and volumetric heat capacity variations versus time (Fig. 6.11(a)) it can be noticed that at the time equal to 60 minutes into the test the soil would have relatively large heat capacity with respect to the volumetric heat capacity of saturated soil, i.e. in the same order of magnitude, when saturation degree is greater than about $22 \%$. This may be considered as a threshold of soil saturation degree for maintaining high heat capacity of the soil for storing larger amount of heat. Also at the same saturation degree, the soil thermal conductivity would still be relatively high at about $1.3 \mathrm{~W} / \mathrm{m} \cdot \mathrm{K}$ with respect to the thermal conductivity of saturated soil, which is about 1.5 W/m.K (Fig. 6.11(b)). From the results, shown in Fig. 6.11(c) with reference to Fig. 6.11(a) and (b), it can be observed that the variations of thermal diffusivity are mainly affected by the variations of heat capacity rather than thermal conductivity, because the variation of heat capacity is at least one order of magnitude, while the thermal conductivity varies within the same order of magnitude over the entire moisture range. Therefore, the thermal diffusivity varies from $3.8 \times$ $10^{-7} \mathrm{~m}^{2} / \mathrm{s}$ at almost saturated conditions to $9.1 \times 10^{-6} \mathrm{~m}^{2} / \mathrm{s}$ at almost dry conditions. The results of the remaining cases, i.e. various temperature levels and temperature differences, are presented in graphical form in Appendix D. 


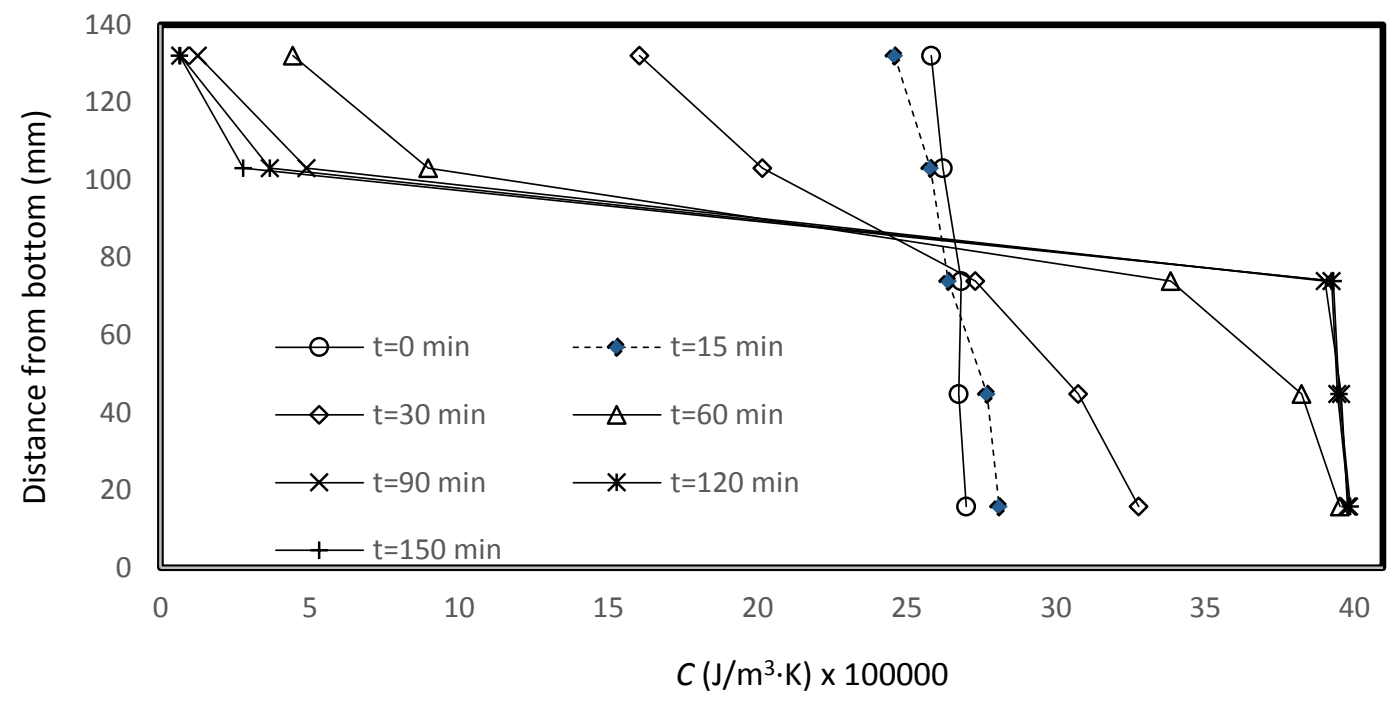

(a)

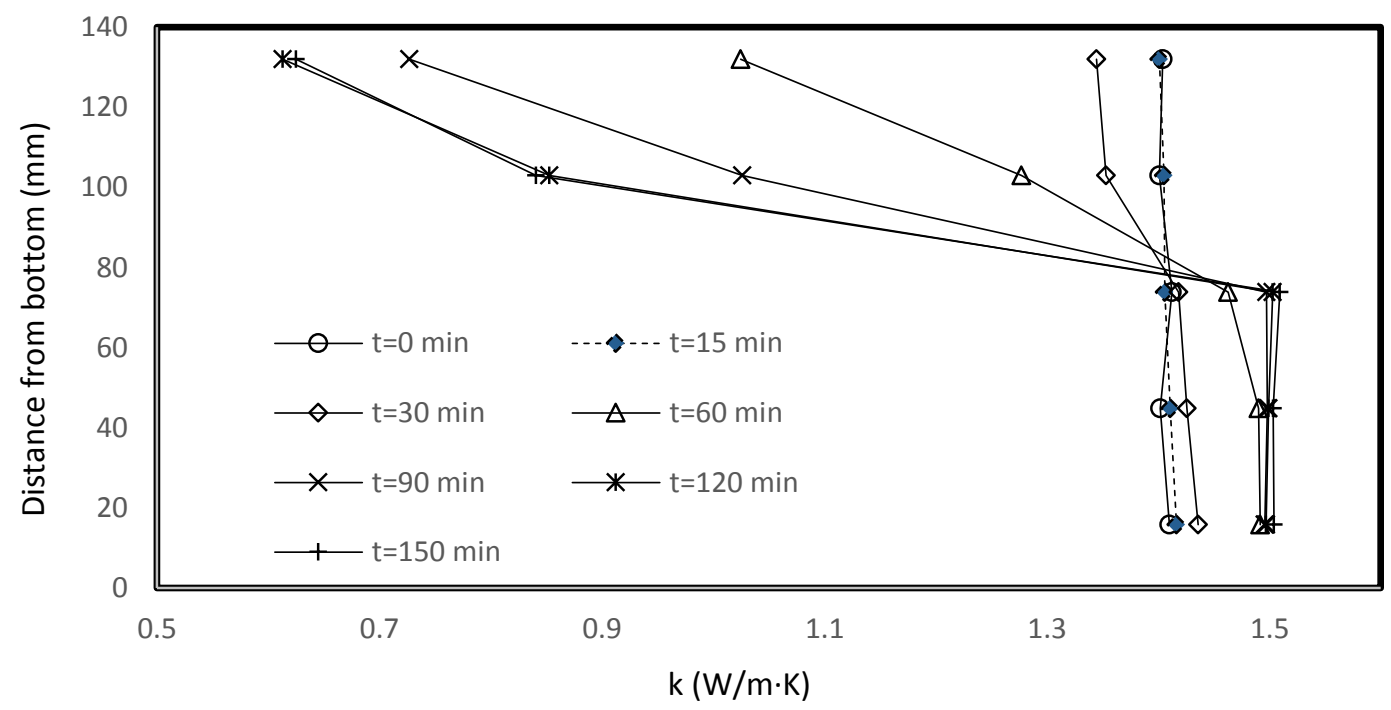

(b)

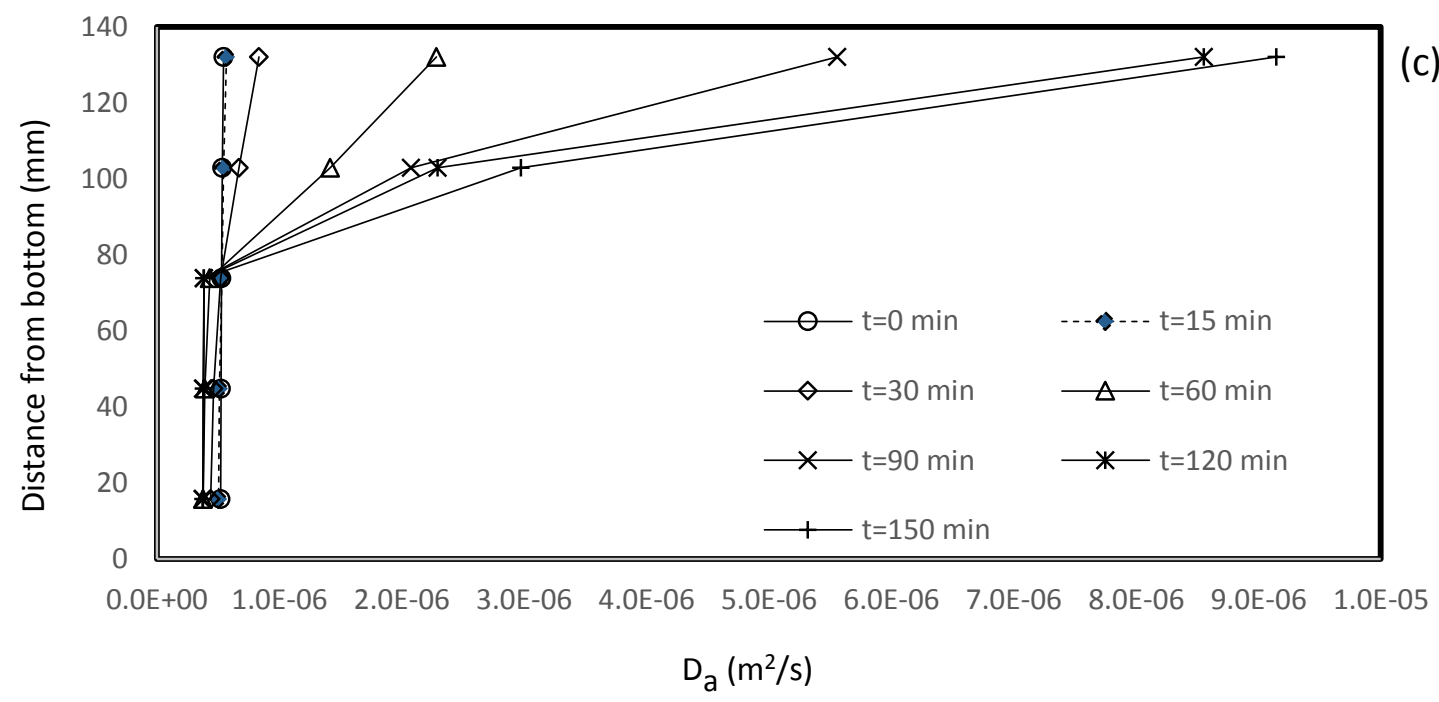

Fig. 6.11 Variations of the thermal properties vs. time along the soil column for the case of 90$10^{\circ} \mathrm{C}$. a) Volumetric heat capacity b) Thermal conductivity c) Thermal diffusivity 


\subsubsection{In-depth Discussion of the Results}

Fig. 6.12 illustrates the variations of the water content differences $\left(\Delta \theta=\theta_{\mathrm{t}}-\theta_{\mathrm{o}}\right)$ over time for the case of $90-10^{\circ} \mathrm{C}$. The water content differences at every time increment were referenced to the water contents of the control volumes at the initial time, i.e. $\theta_{\mathrm{o}}=0.26 \mathrm{~m}^{3} / \mathrm{m}^{3}$ or $\mathrm{S}_{\mathrm{r}}=0.65$. From the results it can be seen that after about 15 minutes, the change in water contents of different control volumes starts to appear. It can be observed that control volumes 1 and 2 lose moisture and at the same time control volumes 4 and 5 gain moisture. Up until 25 minutes into the test, control volume 3 acts as a transit and remain at relatively constant rates of moisture exchange between its preceding and following control volumes. However after about 30 minutes it begins to have net gain of moisture from control volume 2, because control volumes 4 and 5 are becoming near full saturation. It should be noted that the process of moisture loss and gain does not necessarily mean the moisture only leaves the control volume or only enters into it. In the case of moisture gains, the term gain is referred to the fact that the amount of the moisture entering into the control volume is greater than the amount of the moisture leaving the control volume. Similarly in case of moisture loss it is referred to the fact that the amount of the moisture entering into the control volume is less than the amount of the moisture leaving the control volume.

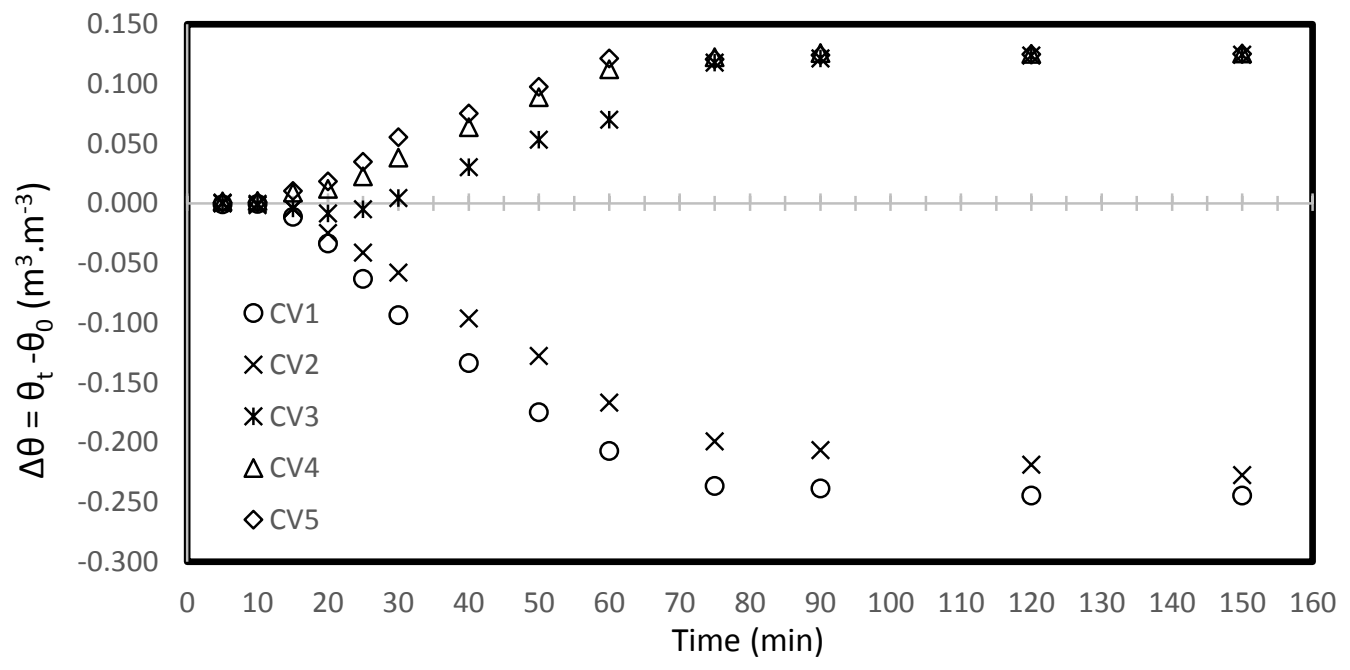

Fig. 6.12 Variations of the water content differences $(\Delta \theta)$ vs. time for the case of $90-10^{\circ} \mathrm{C}$. The symbol $\Delta \theta$ is the water content difference of control volumes between time $t$ and initial time $t_{o}$. The positive value is an indication of moisture gain and the negative value is an indication of moisture loss 
Figs. 6.13 to 6.17 illustrates the variations of the net liquid fluxes $\left(\mathrm{kg} \cdot \mathrm{s}^{-1} \cdot \mathrm{m}^{-2}\right)$, temperature $\left({ }^{\circ} \mathrm{C}\right)$, saturation degrees $(\% \mathrm{Sr})$, temperature gradients $\left({ }^{\circ} \mathrm{C} / \mathrm{m}\right)$ and moisture gradients $\left(\mathrm{m}^{3} \cdot \mathrm{m}^{-3} \cdot \mathrm{m}^{-1}\right)$ over time ( $\mathrm{min}$ ) for control volumes 1 to 5, respectively. The net liquid flux of each control volume is calculated as follows:

$\left(q_{l}\right)_{n e t}=\frac{\theta_{t}-\theta_{t-\Delta t}}{\Delta t} \rho_{l} \Delta x$

where $\theta_{t}$ is the volumetric water content at the present time, $\theta_{t-\Delta t}$ is the volumetric water content at the previous time step, $\rho_{l}$ is the density of liquid water, and $\Delta x$ is the height of the control volume. The volumetric water contents of all control volumes were measured simultaneously with a time interval of 5 minutes, i.e. $\Delta t=5$ minutes. The heights of all control volumes are equal to the height of the soil column divided by 5 , i.e. $\Delta x=29.6 \mathrm{~mm}$.

It is known from the theory of heat and moisture transfer in soils (Philip and de Vries 1957, de Vries 1987) that the three driving forces to result in liquid movement are due to moisture gradient, temperature gradient and gravity.

$q_{l}=-\rho_{l} D_{\theta l} \nabla \theta-\rho_{l} D_{T l} \nabla T-K_{h} \hat{k}$

where $D_{\theta l}$ is moisture diffusivity due to moisture gradient $\left(\mathrm{m}^{2} / \mathrm{s}\right), D_{T l}$ is moisture diffusivity due to temperature gradient $\left(\mathrm{m}^{2} / \mathrm{s} \cdot \mathrm{K}\right)$ and $K_{h}$ is hydraulic conductivity $(\mathrm{m} / \mathrm{s})$.

After a wet soil sample was prepared in the soil cell, it was left to stand for over 24 hours in vertical position before an experiment would begin. Obviously from Fig. 6.10 it can be observed that the moisture was uniform along the soil column; this indicates that the gravity does not play an important role in liquid movement for Matilda soil. Therefore, the main driving forces causing liquid movement are the moisture gradient and temperature gradient; the following equations (Eqs. (6.5) to (6.8)) are used to calculate the temperature gradients and moisture gradients which will help to explain the effects of the gradients on the net liquid flux of each control volume. The temperature gradients at the upper and lower boundaries of each control volume are calculated as follows: 
$\left.\frac{d T}{d x}\right|_{\text {upper }}=\frac{T_{C V u}-T_{C V}}{\Delta x}$

$\left.\frac{d T}{d x}\right|_{\text {lower }}=\frac{T_{C V}-T_{C V l}}{\Delta x}$

where $T_{C V}$ is the temperature of the present control volume, $T_{C V u}$ and $T_{C V l}$ are the temperatures of the upper and lower control volumes, respectively.

The moisture gradients at the upper and lower boundaries of each control volume are calculated as follows:

$\left.\frac{d \theta}{d x}\right|_{\text {upper }}=\frac{\theta_{C V u}-\theta_{C V}}{\Delta x}$

$\left.\frac{d \theta}{d x}\right|_{\text {lower }}=\frac{\theta_{C V}-\theta_{C V l}}{\Delta x}$

where $\theta_{C V}$ is the volumetric water content of the present control volume, $\theta_{C V u}$ and $\theta_{C V l}$ are the volumetric water contents of the upper and lower control volumes, respectively.

In the following, the relationships among the results of each control volume are analyzed. The temperature and moisture gradients are shown for both upper and lower boundaries of each control volume. This helps to have a better understanding of how the heat and moisture enter and exit a specific control volume.

Fig. 6.13 represents the results of control volume 1 for the case of $90-10^{\circ} \mathrm{C}$. In this control volume, the highest temperature was achieved to be $75.6^{\circ} \mathrm{C}$, as shown in Fig. 6.13(b). Since no moisture enters into the control volume from its upper boundary, the moisture gradients remained at zero at the upper boundary. By simultaneously looking at Figs. 6.13(b) and (d), it can be noticed that the increase of temperature results in the increase of temperature gradients. The temperature gradients are greater in the upper boundary compared to the lower boundary. Considering Figs. 6.13(a), (d) and (e), it can be perceived that the increase of temperature gradients induces the increase of moisture fluxes and that would induce the increase in moisture gradients in the lower boundary of the control volume. As the temperature becomes relatively constant after about 90 minutes (Figs. 6.13(b) and (c)), the changes of temperature gradients and net liquid flux would 
also become constant; however, the moisture gradient continues to decrease with time. This indicates that control volume 1 continues to get drier. The liquid flux leaves control volume 1 at a relatively high rate from 10 to 30 minutes into the test with a maximum liquid flux of about 0.003 $\mathrm{kg} \cdot \mathrm{s}^{-1} \cdot \mathrm{m}^{-2}$ at $t=30$ minutes (Fig. 6.13(a)), at which time about half of the water content in control volume 1 has migrated to control volume 2 (Fig. 6.13(c)). At that moment, the temperature gradients at the upper and lower boundaries have reached 1444 and $560^{\circ} \mathrm{C} / \mathrm{m}$ (Fig. 6.13(d)), respectively, which strongly drive the moisture toward control volume 2. Although the moisture gradient at the lower boundary opposes the moisture migration at that moment, it fails to stop the moisture migration because the moisture gradient of $1.33 \mathrm{~m}^{3} \cdot \mathrm{m}^{-3} \cdot \mathrm{m}^{-1}$ (Fig. 6.13(e)) is too small. 

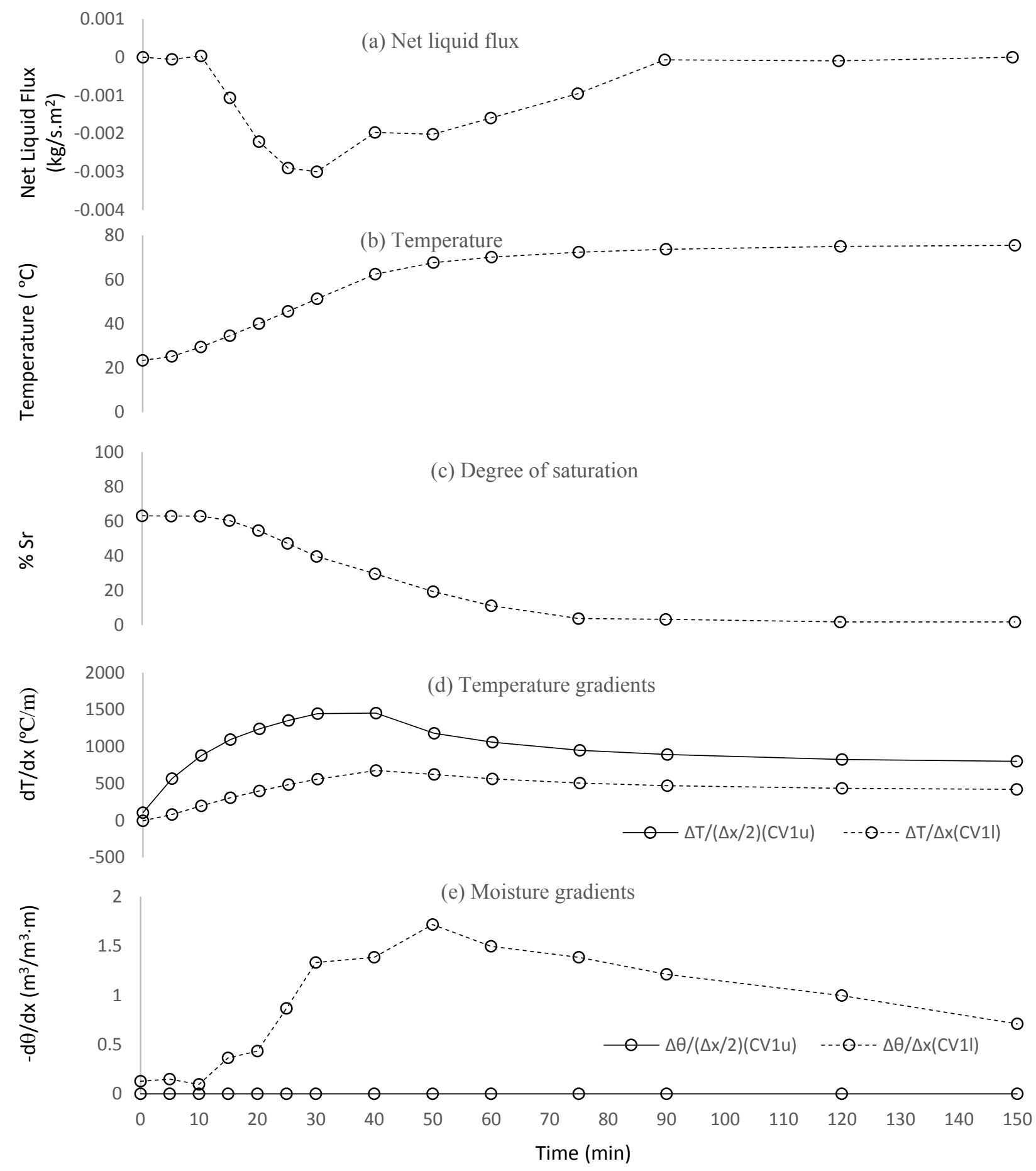

Fig. 6.13 The experimental results for control volume 1 of the soil column for the case of $90^{\circ} \mathrm{C}$ hot plate and $10^{\circ} \mathrm{C}$ cold plate 
Fig. 6.14 represents the results of control volume 2 for the case of $90-10^{\circ} \mathrm{C}$. In this control volume, the highest temperature was achieved to be $63.1^{\circ} \mathrm{C}$, as shown in Fig. 6.14(b). The amount of moisture leaving control volume 1 would enter into the upper boundary of control volume 2. Similar to the previous case, control volume 2 would also get drier over time; however, with relatively lower rate. This can be verified by comparing the values of net liquid fluxes in both control volumes. Considering the results of temperature, saturation degree and temperature gradients, shown in Fig. 6.14(b), (c) and (d), it can be perceived that, as the temperature and temperature gradients increase, saturation degree decreases. In addition, the gravity would also play a role in moving the moisture down toward control volume 3 , because the viscosity and surface tension of water reduces as the temperature rises. Hence, this phenomenon results in higher moisture gradients at the lower boundary of control volume 2. By comparing the moisture gradient variations at the upper and lower boundaries of control volume 2, it can be seen that the moisture gradient at the lower boundary increases much higher than the moisture gradient at the upper boundary. From Fig. 6.14(a), it can be observed that the net liquid fluxes are all negative values, indicating net loss of moisture in control volume 2 at a relatively high rate from 20 to 60 minutes into the test with an average net loss of liquid flux of about $0.0018 \mathrm{~kg} \cdot \mathrm{s}^{-1} \cdot \mathrm{m}^{-2}$ (Fig. 6.14(a)). By 60 minutes into the test, even with the moisture gain from control volume 1, the saturation degree of control volume 2 has dropped from original $64.1 \%$ to $22.3 \%$ due to net loss of moisture to control volume 3 (Fig. 6.14(c)). At the same moment, the temperature gradients at the upper and lower boundaries have reached 564 and $452^{\circ} \mathrm{C} / \mathrm{m}$ (Fig. 6.14(d)), respectively, which drive the moisture toward control volume 3 . In this case, similar to the previous control volume, it can be understood that although the moisture gradient at the lower boundary opposes the moisture migration at the moment, it yet fails to stop the moisture migration even though the moisture gradient is relatively high at $8.2 \mathrm{~m}^{3} / \mathrm{m}^{3} \cdot \mathrm{m}$ (Fig. 6.14(e)). The analysis of control volume 3 is important since it acts as a transit between control volumes 2 and 4 . 

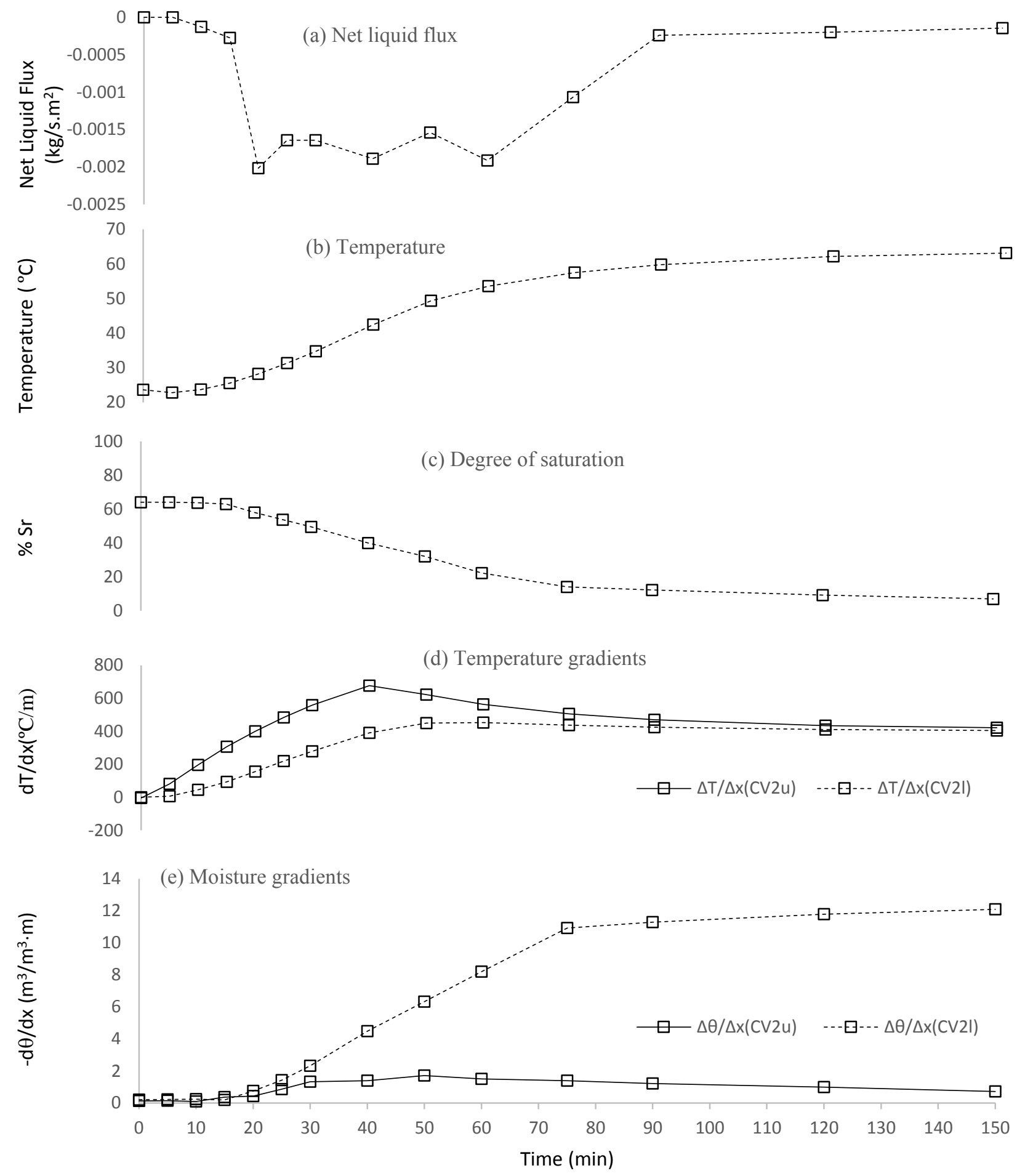

Fig. 6.14 The experimental results for control volume 2 of the soil column for the case of $90^{\circ} \mathrm{C}$ hot plate and $10^{\circ} \mathrm{C}$ cold plate 
Fig. 6.15 illustrates the results of control volume 3 for the case of $90-10^{\circ} \mathrm{C}$. In this control volume, the highest temperature was achieved to be $51.1^{\circ} \mathrm{C}$. Similar to the previous control volume, the moisture leaving control volume 2 enters into control volume 3 . Unlike control volumes 1 and 2, moisture content increases in control volume 3 (Fig. 6.15(c)), due to the net gain of moisture (Fig. 6.15(a)); however, the net gain of moisture is at a lower rate in comparison with control volumes 1 and 2. From Fig. 6.15(e), it can be noticed that after about $75 \mathrm{~min}$, the variations of the moisture gradients become very small. In fact, the moisture gradient at the lower boundary becomes negligibly small, indicating that both control volumes 3 and 4 have achieved similar moisture contents which are close to the saturation point of the soil at saturation degrees of about 95-96\% (Figs. 6.15(c) and 6.16(c)). From Fig. 6.15(a), it can be observed that the net gain of liquid flux in control volume 3 is at relatively high rates from 25 to 75 minutes into the test with a maximum liquid flux of about $0.0016 \mathrm{~kg} \cdot \mathrm{s}^{-1} \cdot \mathrm{m}^{-2}$ at $t=75$ minutes. At the same moment, the temperature gradients at the upper and lower boundaries have reached 438 and $391^{\circ} \mathrm{C} / \mathrm{m}(\mathrm{Fig}$. 6.15(d)), respectively, which continue to drive small amount of the moisture toward control volume 4. In this case, it can be seen that although the moisture gradient at the upper boundary opposes the moisture migration from control volume 2 to control volume 3 at that moment, it still cannot completely stop the migration of the moisture even though the moisture gradient is high at $10.9 \mathrm{~m}^{3} \cdot \mathrm{m}^{-3} \cdot \mathrm{m}^{-1}$ (Fig. 6.16(e)). Fig. 6.16 demonstrates the results of control volume 4 for the case of $90-10^{\circ} \mathrm{C}$. In this control volume, the highest temperature was achieved to be $39.6^{\circ} \mathrm{C}$. Considering Fig. 6.16(d) and (e), it can be observed that the highest moisture gradient happens at 60 minutes and the highest temperature gradient happens at 75 minutes. However, that is not necessarily the case in control volume 5 (Fig. 6.17(d) and (e)). Indeed the regions with higher moisture contents have less potential in accepting the incoming moisture. Beside the above reason, it should be noted that some moisture might have been evaporated into vapor at high-temperature regions which cannot be detected with the applied technique in this study. The liquid flux enters control volume 4 at a relatively high rate from 20 to 30 minutes into the test with a maximum liquid flux of about $0.00356 \mathrm{~kg} \cdot \mathrm{s}^{-1} \cdot \mathrm{m}^{-2}$ (the sum of net liquid fluxes for control volumes 4 and 5) at $t=30$ minutes (Figs. 6.16(a) and 6.17(a)). At the moment, the temperature gradients at the upper and lower boundaries have reached 204 and $139^{\circ} \mathrm{C} / \mathrm{m}$ (Fig. 6.16(d)), respectively, which drive the moisture toward control volume 5 at a relatively high rate of $0.002 \mathrm{~kg} \cdot \mathrm{s}^{-1} \cdot \mathrm{m}^{-2}$ (Fig. 6.17(a)) even though the temperature gradients are not very high in comparison to the temperature gradients in other 
previous control volumes. From Figs. 6.16(c) and 6.17(c), it can be observed that control volume 4 become relatively saturated, i.e. $\theta_{\mathrm{o}}=0.385 \mathrm{~m}^{3} / \mathrm{m}^{3}$ or $\mathrm{S}_{\mathrm{r}}=0.96$, from 60 to 75 minutes into the test. Within this period, the net liquid fluxes in both control volumes 4 and 5 become almost zero (Figs. 6.16(a) and 6.18(a)). From Fig. 6.17(c) it can be seen that during above period control volume 5 remain relatively saturated. Therefore, the moisture potential become very small in control volume 5 after 60 minutes into the test. This condition prevents the moisture migration from control volume 4 and so, results in rapid reduction in moisture gradients (Figs. 6.16(e) and 6.17(e)). 

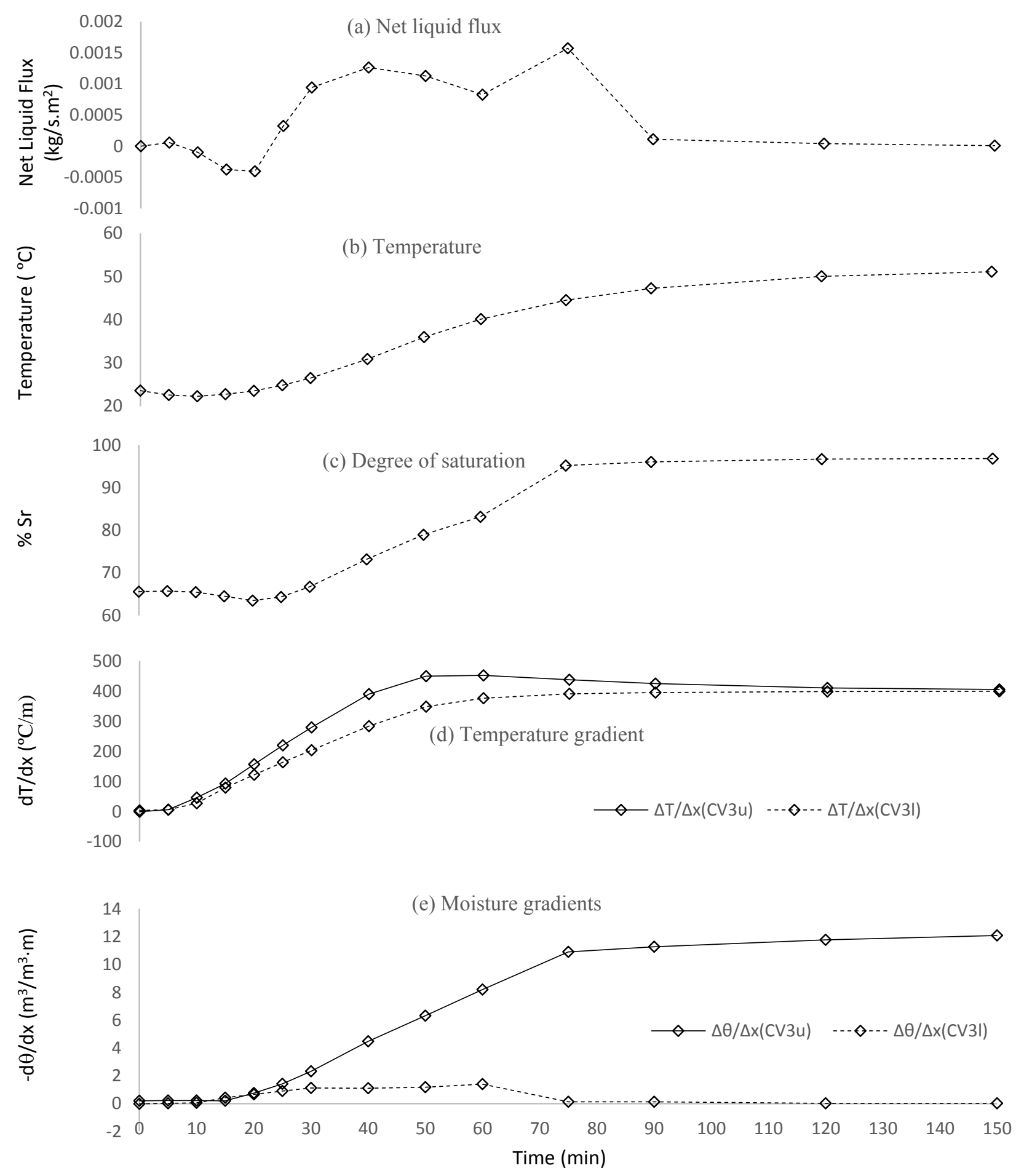

Fig. 6.15 The experimental results for control volume 3 of the soil column for the case of $90^{\circ} \mathrm{C}$ hot plate and $10^{\circ} \mathrm{C}$ cold plate 

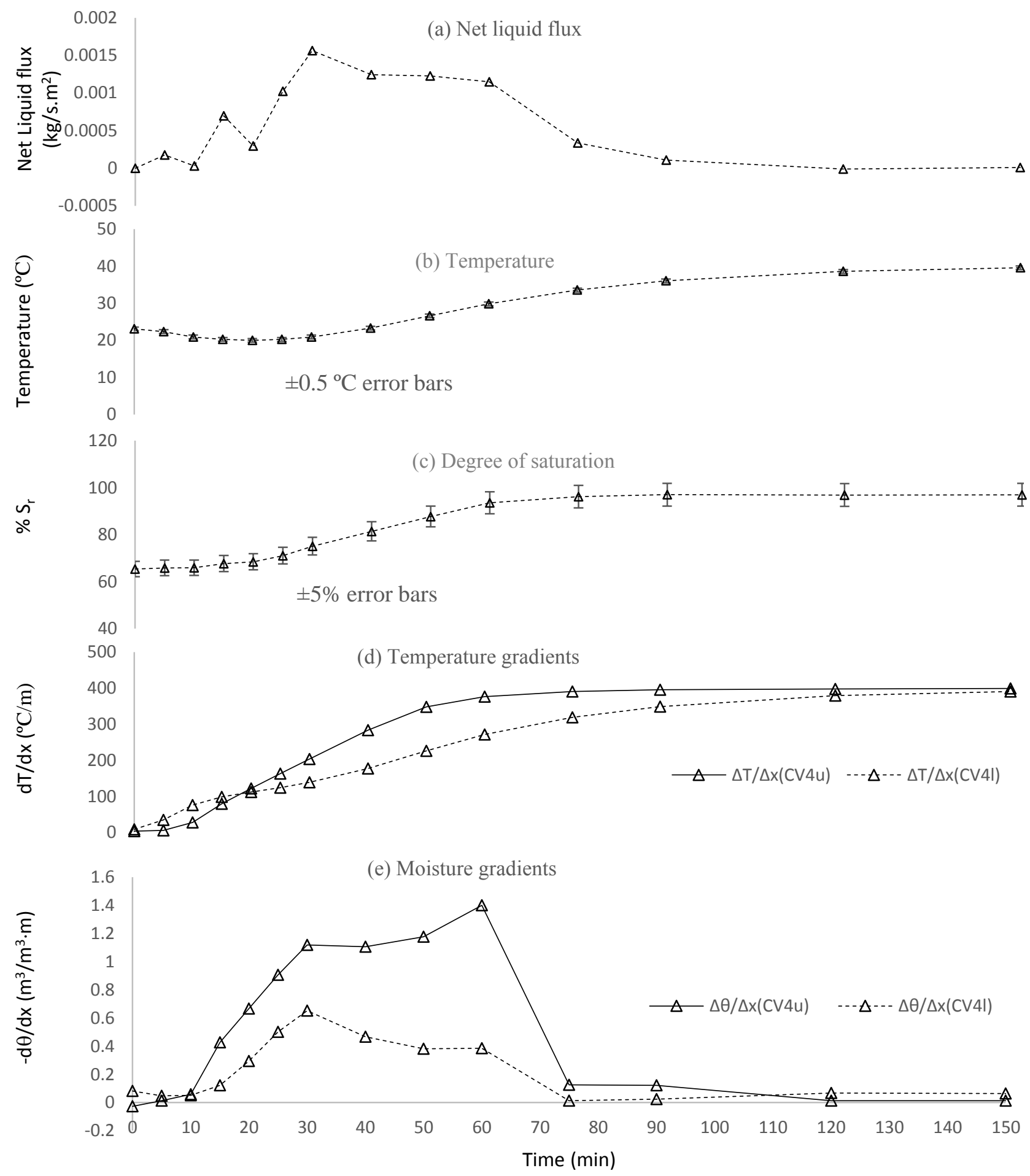

Fig. 6.16 The experimental results for control volume 4 of the soil column for the case of $90^{\circ} \mathrm{C}$ hot plate and $10^{\circ} \mathrm{C}$ cold plate 

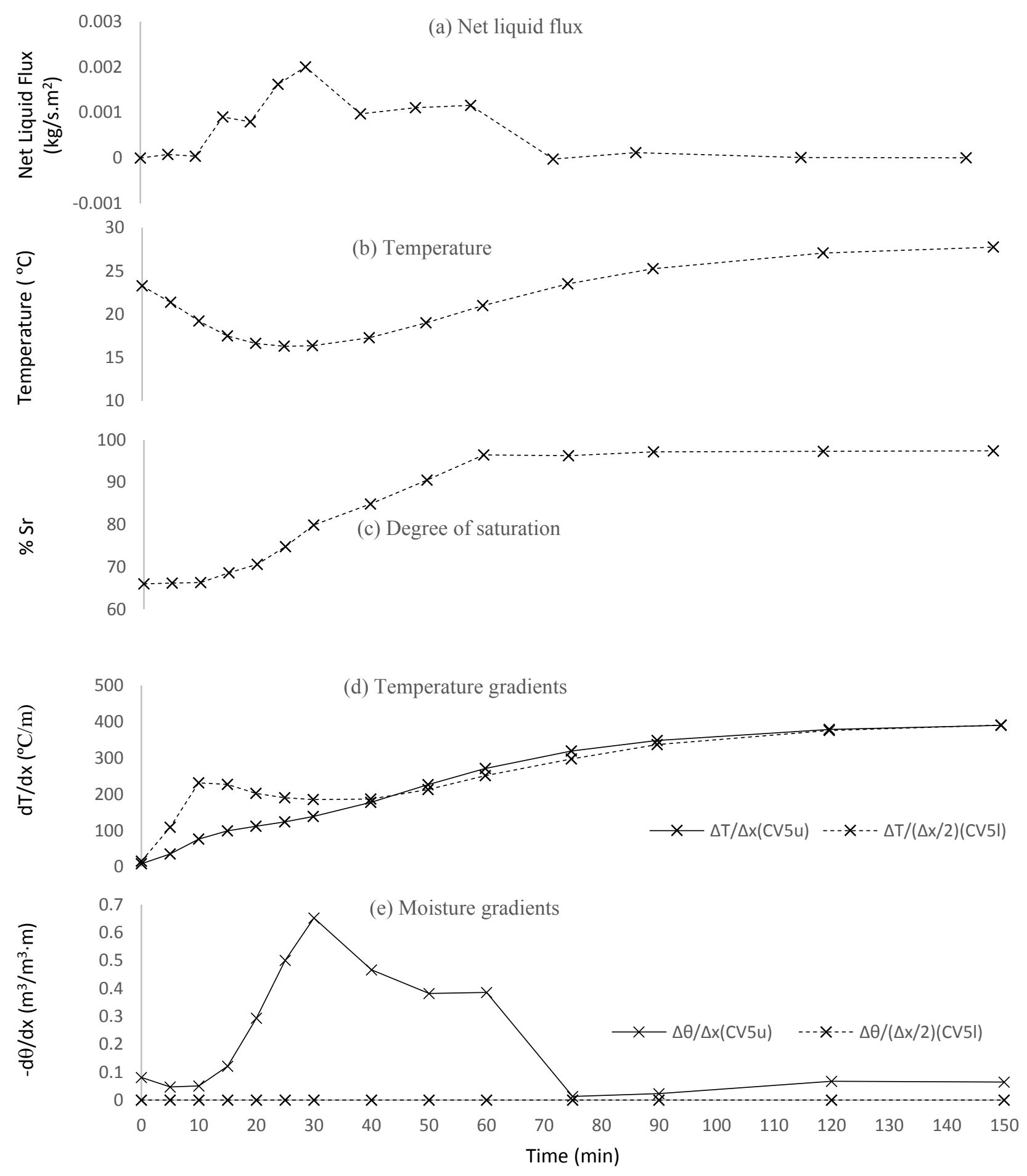

Fig. 6.17 The experimental results for control volume 5 of the soil column for the case of $90^{\circ} \mathrm{C}$ hot plate and $10^{\circ} \mathrm{C}$ cold plate 


\subsection{Results Summary}

One-dimensional heat and moisture transfer was studied within a vertical soil column to investigate heat and moisture transfer characteristics in a soil (Matilda) at high temperatures. The results were presented for both dry and wet soils. For the case of dry soil, temperature distributions and heat gains/losses at the steady-state conditions along the soil cell at the various temperature levels were investigated.

The highest temperature deviation from the linear temperature profile was found to be $18.6 \%$ at the temperature level of $82.6^{\circ} \mathrm{C}$ and the temperature gradient of about $90^{\circ} \mathrm{C} / \mathrm{m}$ which occur at around $40 \mathrm{~mm}$ from the top of the soil column. It was observed that there was higher heat losses in the upper portion of the soil cell due to the higher temperature difference between the upper portion temperature and the room temperature. For the case of wet soil, it was observed that the highest difference of heat fluxes between the top and bottom HFMs would not exceed $4.2 \%$ and the highest temperature deviation from the LTP in the worst case is not more than 7.7\%. The amount of heat loss with respect to the heat transfer through the soil was found to be relatively small; therefore, greater uniformity of heat flux in the radial direction would be achieved along the soil column. Also it was observed that the higher discrepancies of the results occur at higher temperature levels while the temperature differences remain relatively similar.

From the results of thermal properties variations vs. time along the soil column, for the case of $90-10^{\circ} \mathrm{C}$, it was observed that the volumetric heat capacity and thermal conductivity increase as moisture content increases; however, thermal diffusivity decreases as moisture content increases. Comparing the results of moisture variations and volumetric heat capacity variations it was noticed that the soil would have relatively large heat capacity with respect to the volumetric heat capacity of saturated soil, i.e. in the same order of magnitude, when saturation degree is greater than about $22 \%$. This may be considered as a threshold of soil saturation degree for maintaining high heat capacity of the soil for storing larger amount of heat. Also at the same saturation degree, the soil thermal conductivity would still be relatively high at about $1.3 \mathrm{~W} / \mathrm{m} \cdot \mathrm{K}$ with respect to the thermal conductivity of saturated soil, which is about $1.5 \mathrm{~W} / \mathrm{m} \cdot \mathrm{K}$. 
From the results of the case of $90-10^{\circ} \mathrm{C}$, it was noticed that, until 30 minutes into the test, the lower region of the soil column was not yet significantly affected by the heat flux from the upper side. It was observed that the heat would take about an hour to reach the bottom of the soil column for a temperature difference of $65.4^{\circ} \mathrm{C}$. Although the focus of result presentation was based on the case of $90-10^{\circ} \mathrm{C}$, the results of the remaining cases were presented in Appendix D in graphical form.

As a general observation, as shown in Fig. 6.18, considering control volume 1 for the three cases of $70-10^{\circ} \mathrm{C}, 80-10^{\circ} \mathrm{C}$ and $90-10^{\circ} \mathrm{C}$, it can be perceived that the increase of the temperature gradient $(d T / d x)$ is the key factor inducing a rapid moisture transfer out from the control volume 1 , resulting in very low moisture content at high temperature gradients. Also, as a general observation, considering control volume 1 for the cases of $45-30^{\circ} \mathrm{C}, 60-45^{\circ} \mathrm{C}, 75-60^{\circ} \mathrm{C}$ and 90 $75^{\circ} \mathrm{C}$, it can be understood that the increase of the temperature level would result in similar moisture contents in the end if the temperature gradients remain about the same (similar $d T / d x)$.

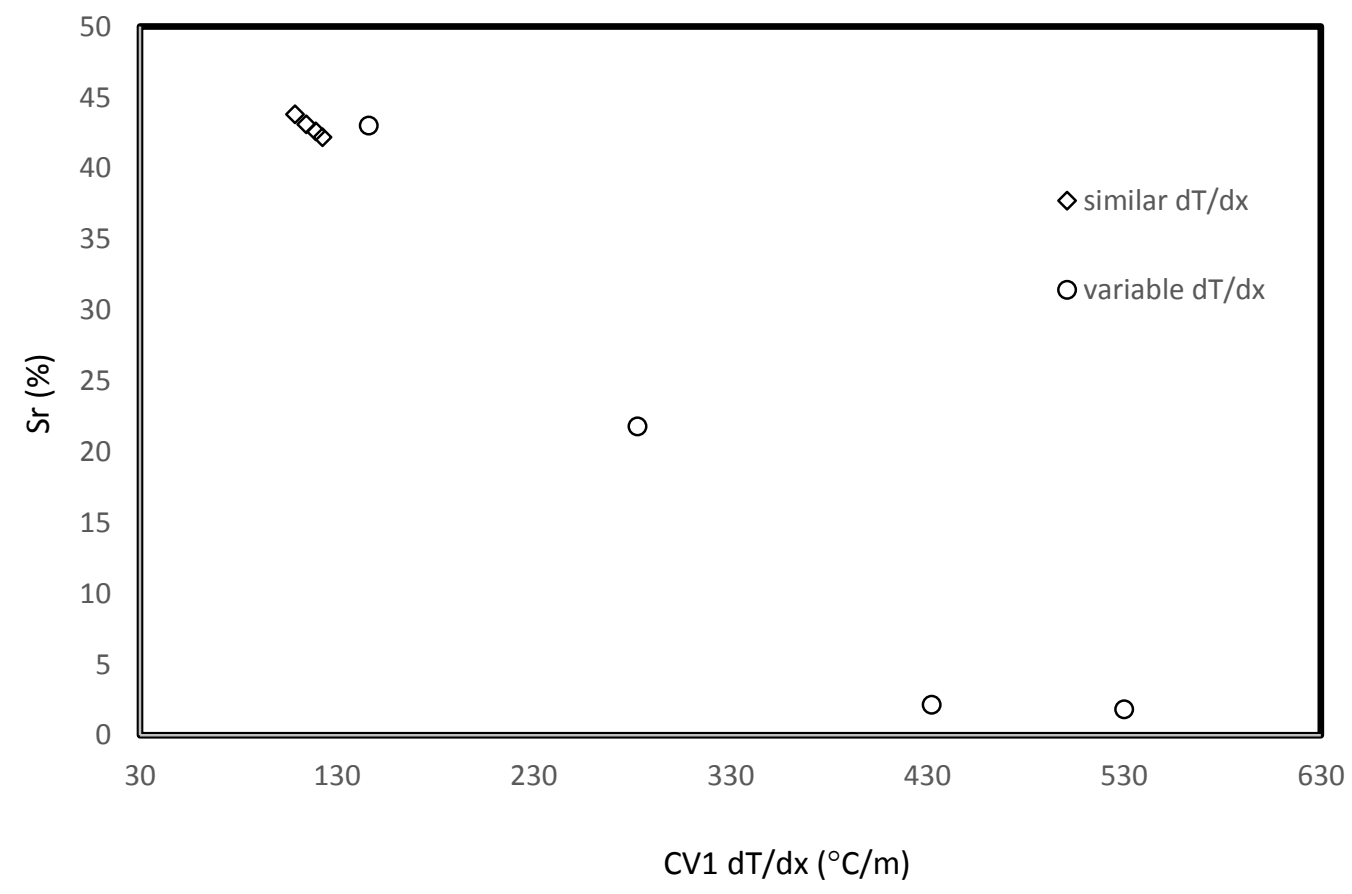

Fig. 6.18 Variations of the saturation degrees in percent vs. temperature gradients at control volume 1 


\section{CHAPTER 7}

\section{CONCLUSIONS AND FUTURE WORK}

\subsection{Introduction}

Through literature survey, it has been found that no comprehensive study on simultaneous heat and moisture transfer in soils had been experimentally conducted at high temperature levels above $40^{\circ} \mathrm{C}$. The objective of this study was to design and build an experimental apparatus for studying heat and moisture transport phenomena in soils at temperatures greater than $40^{\circ} \mathrm{C}$ up to $90^{\circ} \mathrm{C}$. An experimental soil cell made of stainless-steel tube with $63.5-\mathrm{mm}$ inner diameter and $151-$ mm height was designed and constructed for experimental studies of one-dimensional heat and moisture transfer within a vertical soil column. The soil cell was exposed to differential heating by sandwiching it between two heated plates for studying heat and moisture transfer in the soil column at different temperature levels and temperature differences. The methodology used in the study to measure temperature, moisture content and thermal properties of soil is the thermo-time domain reflectometry (T-TDR) which is a combination of two different techniques: time domain reflectometry (TDR) and heat pulse. Five in-house-made T-TDR probes were inserted along the soil cell in order to simultaneously measure spatial and temporal variations of soil temperatures, moisture contents and thermal properties, such as thermal diffusivity, thermal conductivity and volumetric heat capacity. The study includes four main parts: first to evaluate the interference effects between two adjacent TDR probes, second to design the experimental apparatus using a numerical method, third to build and assess the reliability and accuracy of the apparatus and experimental techniques, and fourth to experimentally investigate heat and moisture transfer characteristics of Matilda soil (loamy sand). After the reliability of the apparatus was assessed, nine cases of the wet soil were studied; they were four temperature levels up to $90^{\circ} \mathrm{C}$ with the same temperature difference of $12.6^{\circ} \mathrm{C}$ (or temperature gradient of $85^{\circ} \mathrm{C} / \mathrm{m}$ ) and five temperature differences from about $20^{\circ} \mathrm{C}$ to $65^{\circ} \mathrm{C}$ (or temperature gradients from about 133 to $433^{\circ} \mathrm{C} / \mathrm{m}$ ) with five mean temperature levels from about $40^{\circ} \mathrm{C}$ to $55^{\circ} \mathrm{C}$. The results show that the temperature gradient is the main driving force to cause moisture migration. For the case of high temperature level and temperature gradient, i.e. $82.1{ }^{\circ} \mathrm{C}$ and $433^{\circ} \mathrm{C} / \mathrm{m}$, the maximum liquid flux can reach as high as $0.003 \mathrm{~kg} \cdot \mathrm{s}^{-1} \cdot \mathrm{m}^{-2}$, during the transient heat and moisture transfer processes. 


\subsection{Contributions}

The major contributions of this experimental study are as follows:

The design and construction of an apparatus for the simultaneous study of heat and moisture transfer in soils: The apparatus, including its data acquisition system and control system, can be utilized for further research investigations of heat and moisture transfer in soils. This includes different types of soils at various levels of temperature and moisture conditions.

The evaluation of TDR interference between the two adjacent TDR probes: The results of this evaluation provided an important information regarding the appropriate spacing between two TDR probes for minimizing their interference effects; hence, this leads to the present design of a soil cell with five TDR probes for small soil volumes.

The experimental results of heat and moisture transfer in a soil (loamy sand) at high temperatures: This results may be used in applications such as the design of high-temperature ground thermal energy storages and other geothermal applications.

\subsection{Concluding Remarks}

The concluding remarks from the results of the numerical simulation and experimental evaluation of a soil cell which was designed, constructed and used in this study are as follows:

A computer software COMSOL was used to properly design the experimental apparatus. Through the numerical study, four stages of design analysis were carried out to eventually reach a satisfactory design which deemed to meet the research objective, i.e. less than 5\% variation of heat fluxes in radial direction along the soil cell. The experimental assessment of the final soil cell (modified model \#3) was first performed using dry Matilda soil. At steady-state conditions, the measured temperature profile along the soil cell deviated from the linear temperature profile by as much as $18.6 \%$, with respect to the overall temperature difference of the soil cell, when the temperature level and gradient of the soil cell was high at $82.6^{\circ} \mathrm{C}$ and $90^{\circ} \mathrm{C} / \mathrm{m}$, respectively. This was due to the bypassed heat loss from the soil to the stainless steel wall; therefore, the difference of heat fluxes between the top and bottom heat flux meters was recorded to be $34 \%$. This case is considered to have the worst uncertainties or differences, because the dry soil has very low thermal 
conductivity which leads to a low heat flux of $26 \mathrm{~W} / \mathrm{m}^{2}$, and it is sensitive to heat loss. The experimental assessment of the final soil cell was also done for a wet Matilda soil at a degree of saturation of about $65 \%$. At steady-state conditions, the measured temperature profile along the soil cell had a maximum deviation of only $7.7 \%$ from the linear temperature profile, with respect to the overall temperature difference of the soil cell, even when the temperature level of the soil cell was high at $82.1^{\circ} \mathrm{C}$. At this condition, the difference of heat fluxes between the top and bottom heat flux meters was recorded to be $4.2 \%$. Unlike the case of dry soil which has a relatively constant thermal conductivity, the temperature deviation in the case of wet soil could be caused by the variation of soil thermal conductivity as a result of variation of water content. The main reason for a much smaller temperature deviation is due to much higher thermal conductivity of the wet soil with respect to those of dry soil $\left(1.4 \mathrm{vs} .0 .26 \mathrm{~W} / \mathrm{m}^{2} \cdot \mathrm{K}\right)$; therefore, the heat loss via the stainless steel wall is relatively small compared to the heat transfer through the wet soil, leading to only $4.2 \%$ difference of heat fluxes between the top and bottom heat flux meters. The percent difference of heat fluxes increases as temperature level increases, and the percent difference of heat fluxes decreases as temperature difference increases. For ground thermal energy storages, a soil with high water content is desirable due to its high thermal capacity and thermal conductivity. Therefore, it is the most important to be able to achieve small experimental uncertainty for the wet soils.

The concluding remarks from the results of TDR interference evaluation are as follows:

The interference effect between two proximate TDR probes was examined for three types of soils, namely FSJ\#1 (silty clay), Matilda (loamy sand) and Ottawa sand C-190 (sand). It was observed that the distance between the probes, soil texture and the water content of the testing medium are the key parameters which affect the interference and TDR probe capability of measurement.

Parallel TDR probes can interfere with each other if the distance between them is around $1 \mathrm{~cm}$. For the samples with higher water content, the effect of interference on the electromagnetic waveform signals is more prominent.

The soils with more clay content are more susceptible to interference, resulting in significant TDR measurement uncertainty of water content. For FSJ\#1 (silty clay), it was observed that, during the event of interference, increase in soil water content induces higher measurement uncertainty of the 
TDR probes up to about $15 \%$ at full saturation conditions. Based on these findings, a total of five TDR probes were utilized to study the heat and moisture transfer in the soil cell, resulting a spacing of $1.76 \mathrm{~cm}$ between the closest needles of the probes for minimizing any effect of interference.

The concluding remarks from the results of heat and moisture transfer within wet soil column are as follows:

Samples of wet Matilda soil at about $65 \%$ degree of saturation were prepared and packed into the stainless steel soil cell. The soil cell was tested vertically with the hot plate on the top and cold plate at the bottom. Nine cases of temperature settings were studied according to the temperatures of the hot and cold plates as the following:

- Similar temperature difference of $15^{\circ} \mathrm{C}: 45-30^{\circ} \mathrm{C}, 60-45^{\circ} \mathrm{C}, 75-60^{\circ} \mathrm{C}$, and $90-75^{\circ} \mathrm{C}$

- Similar temperature level of $40^{\circ} \mathrm{C}: 50-30^{\circ} \mathrm{C}, 60-20^{\circ} \mathrm{C}, 70-10^{\circ} \mathrm{C}, 80-10^{\circ} \mathrm{C}$, and $90-10^{\circ} \mathrm{C}$

The soil cell was subdivided into five equal-height control volumes of $29.6 \mathrm{~mm}$ each. There was a T-TDR probe inserted into the center of each control volume. A total of 10 thermocouples in the T-TDR probes were used to simultaneously measure temperatures along the soil cell in every second, together with two temperatures measured by the thermocouples embedded in the heat flux meters. Soil thermal properties and volumetric water content were measured by the heat pulse technique in 5-minute intervals up to 30 minutes into the tests, then 10-minute intervals up to 60 minutes, then 15-minute intervals up to 90 minutes, and finally 30-minute interval up to 150 minutes.

Temperature, degree of saturation, net liquid flux, temperature gradients, and moisture gradients of each control volume were presented in Chapter 6 and Appendix D in graphical form. As an example, the most drastic and interesting case of $90-10^{\circ} \mathrm{C}$ is concluded as follows:

The steady state condition was obtained after about 120 minutes into the test. Due to large initial temperature difference (about $65^{\circ} \mathrm{C}$ ) between the hot plate and the top part of the soil, the temperature rise at the points closer to the upper hot plate is more rapidly than the temperature drop at the points closer to the lower cold plate, which has only about $11^{\circ} \mathrm{C}$ difference between the cold plate and the bottom part of the soil. 
The heat would take about 30 to 60 minutes to reach the bottom of the soil column for a temperature difference of about $65^{\circ} \mathrm{C}$.

The moisture contents in the two upper control volumes (control volumes 1 and 2) dramatically dropped over time; while in the two lowest control volumes (control volumes 4 and 5), their moisture contents correspondingly increased. By comparing the results of moisture variations with the results of temperature variations, it can be understood that the significant increase of the temperature gradients in control volumes 1 and 2 was the key factor which induced the rapid moisture transfer out from the control volumes.

In control volume 1 , the highest temperature was achieved to be $75.6^{\circ} \mathrm{C}$. The increase of temperature gradients induces moisture fluxes flowing downward. As the temperature becomes relatively constant, the changes of temperature gradients and net liquid flux would also become constant. The liquid flux leaves control volume 1 at a relatively high rate from 10 to 30 minutes into the test with a maximum liquid flux of about $0.003 \mathrm{~kg} \cdot \mathrm{s}^{-1} \cdot \mathrm{m}^{-2}$ at $t=30$ minutes at which time about half of the water content in control volume 1 has migrated to control volume 2 . At the moment, the temperature gradients at the upper and lower boundaries have reached 1444 and $560^{\circ} \mathrm{C} / \mathrm{m}$, respectively, which strongly drive the moisture toward control volume 2 . Eventually, the moisture content of control volume 1 decreases to $1.71 \%$ degree of saturation at 150 minutes into the test.

In control volume 2 , the highest temperature was achieved to be $63.1^{\circ} \mathrm{C}$. The gravity would also play a role in moving the moisture down toward control volume 3 , because the viscosity and surface tension of water reduces as the temperature rises. Hence, this phenomenon results in higher moisture gradients at the lower boundary of control volume 2, as the moisture content in control volume 3 reaches near full saturation. At the same time, the moisture gradient reaches a high value of about $12.0 \mathrm{~m}^{3} / \mathrm{m}^{3} \cdot \mathrm{m}$, which opposes the temperature gradient of about $410^{\circ} \mathrm{C} / \mathrm{m}$, and reduces the liquid flux from 0.002 to $0.0002 \mathrm{~kg} \cdot \mathrm{s}^{-1} \cdot \mathrm{m}^{-2}$. The final moisture content of control volume 2 is $7 \%$ degree of saturation at 150 minutes into the test.

In control volume 3 , the highest temperature was achieved to be $51.1^{\circ} \mathrm{C}$. The net liquid fluxes, in control volume 3 , reduces more in comparison with control volumes 1 and 2 because its moisture 
content reaches almost full saturation (96\% degree of saturation) at around 75 minutes into the test.

In control volume 4 , the highest temperature was achieved to be $39.6^{\circ} \mathrm{C}$. Similar to control volume 3 , its moisture content also reaches near full saturation at 75 minutes into the test; therefore, the liquid flux from control volume 3 also becomes very low at $0.00034 \mathrm{~kg} \cdot \mathrm{s}^{-1} \cdot \mathrm{m}^{-2}$.

Finally, in control volume 5, the highest temperature was achieved to be $27.8^{\circ} \mathrm{C}$. It gains liquid from control volume 4 at a relatively high rate up to $0.002 \mathrm{~kg} \cdot \mathrm{s}^{-1} \cdot \mathrm{m}^{-2}$ at 30 minutes into the test. After that, it continues to gain moisture at a relatively constant rate of $0.001 \mathrm{~kg} \cdot \mathrm{s}^{-1} \cdot \mathrm{m}^{-2}$ until 60 minutes into the test, at which time the moisture content of control volume 5 reaches almost full saturation. Then, the liquid flux drops drastically to $0.0001 \mathrm{~kg} \cdot \mathrm{s}^{-1} \cdot \mathrm{m}^{-2}$, and eventually it reduces to $9.8 \times 10^{-6} \mathrm{~kg} \cdot \mathrm{s}^{-1} \cdot \mathrm{m}^{-2}$ at 120 minutes into the test.

\subsection{Future Work}

Precise modeling of coupled heat and moisture transfer in high-temperature ground thermal storages is yet to be realized, and so it requires further studies. The apparatus which was designed and constructed in the present study can be used for any future studies of heat and moisture transfer processes for other types of soils and with different degrees of saturation. More comprehensive studies can be performed based on the following future experimental results:

- Different heating conditions, e.g. heating from below,

- Different orientations of the soil cell, e.g. horizontal position,

- Different processes in series, e.g. first heating from above and then followed by heating from below. 


\section{APPENDIX A}

\section{A LIST OF MATERIALS}

\section{A.1 List of Material for construction of T-TDR Probe}

Needles: 18 gauge hypodermic tubing

Specifications: Stainless steel 304 OD: $1.27 \mathrm{~mm} \&$ ID: $0.84 \mathrm{~mm}$. Length: $914.4 \mathrm{~mm}$ Quantity: 1

Price: $\$ 12$

Company: www.amazon.com

Heater Wire: 38 gauge Nichrome 80 wire with enamel coating

Available 5 foot for $\$ 45$ USD at www.wiretron.com

Thermo Couple:

Specifications: TT-T-40-SLE OMEGA Thermocouple ${ }^{14}$

Quantity: 125 foot spool

Price per unit: \$ 52/unit total \$ 52 CAD

Company: www.omega.com use Canada branch

Needle fillers: Omegabond Resin \& Catalyst 101

Specifications: OB-101-16

Quantity: 1 pounds (can) includes two parts epoxy

Price: \$ 70 USD

Company: www.omega.com

Release agent for molding: Miller-stephenson PTFE Release Agent Dry Lubricant

Company: www.miller-stephenson.com

Note: To Make the purchase please call : 800-307-2199

280 Belfield RD. ask for MS-122AD

Epoxy for the probe Head: United Resin El-Cast with 641 hardener.

${ }^{14}$ Fine Wire Duplex Insulated Thermocouple Wire (http://www.omega.com/techref/colorcodes.html) 
Specification: Cure at room temperature with maximum use temperature of $250^{\circ} \mathrm{F}$

Ratio of hardener to Resin is 1 to 5

Company: http://www.unitedresin.com/index.html

Coaxial Cable:

Specification: RG59 75 Ohm Flexible Coax Cable Black PVC Jacket 25 foot Price: \$ 30.25 USD

Company: www.fairviewmicrowave.com

Coaxial connectors: UPL220-004 Trompeter Coaxial Cable connectors

Specification: 75 Ohm BNC Male Connector Crimp/Solder Attachment for RG59 cable Price: \$7.57 USD per unit for minimum 10 unit price is \$75.7 USD

Company: www.fairviewmicrowave.com

28 AWG ribbon cable

Require at least 20 foot

Company: http://www.digikey.ca/product-search/en/cables-wires/flat-ribbon-cables

Soldering equipment part B: Copper Bond Flux

Specification: Kapp Copper-Bond ${ }^{\mathrm{TM}}$ Flux $500^{\circ} \mathrm{F}-750^{\circ} \mathrm{F}\left(260^{\circ} \mathrm{C}-390^{\circ} \mathrm{C}\right)$

Price: \$ 10 USD for 2 OZ $(60 \mathrm{ml})$ bottle \& 6 OZ $(180 \mathrm{ml}) \$ 20$ USD

Company: http://www.solderdirect.com/all-products/companion-fluxes/kapp-copperbondtm-flux-500-f-750-f-260-c-390-c.html

\section{A.2 List of Material for Apparatus Construction}

Aluminum Alloy_2024 plate $8 " \times 8 " \times \frac{1 "}{2}$

Quantity \& price: 2 for 135.58 CAD

Company: www.McMaster.com

Aluminum Alloy_2024 plate $8 " \times 8 " \times \frac{1 "}{4}$

Quantity \& price: 2 for 84.28 CAD

Company: www.McMaster.com

Aluminum Alloy_2024 sheet $6 " \times 6 " \times \frac{1}{16}$ 
Quantity \& price: 2 for 15.7 CAD

Company: www.McMaster.com

Stainless Steel tube 304 ID 2.5", OD 3" and Length $\frac{1^{\prime}}{2}$

Quantity \& price: 1 for 54.14 CAD

Company: www.McMaster.com

Stainless Steel tube 304 ID 5.5", OD 6" and Length $\frac{1^{\prime}}{2}$

Quantity \& price: 1 for 133.5 CAD

Company: www.McMaster.com

Silicone O-Ring Length $10^{\prime}$

Quantity \& price: 1 spool for 3.7 CAD

Company: www.McMaster.com

Loctite Silicone sealants $2.7 \mathrm{oz}$

Quantity \& price: 1 tube for 5.39 CAD

Company: www.McMaster.com

Type 303 Stainless steel Barbed tube fitting $90^{\circ}$ elbow for $\frac{3^{\prime \prime}}{8}$ tube ID male pipe size

18 Threads per inch, $0.41^{\prime \prime}$ Thread Engagement-Withstands up to $750^{\circ} \mathrm{F}$

Quantity \& price: 4 for 32 CAD

Company: www.McMaster.com

Standard Heat Flux sensor $60 \mathrm{~mm}$ with sensitivity of $15 \mathrm{microVolt} / \mathrm{Watts} / \mathrm{m}^{2}$

Built in-T type thermocouples

Quantity \& price: 2 for 532 EUR, Captec Enterprise co.-France 


\section{APPENDIX B}

\section{ENGINEERING CAD DRAWINGS}

\section{B.1 Polyamid Plastic Mold for T-TDR Probe}
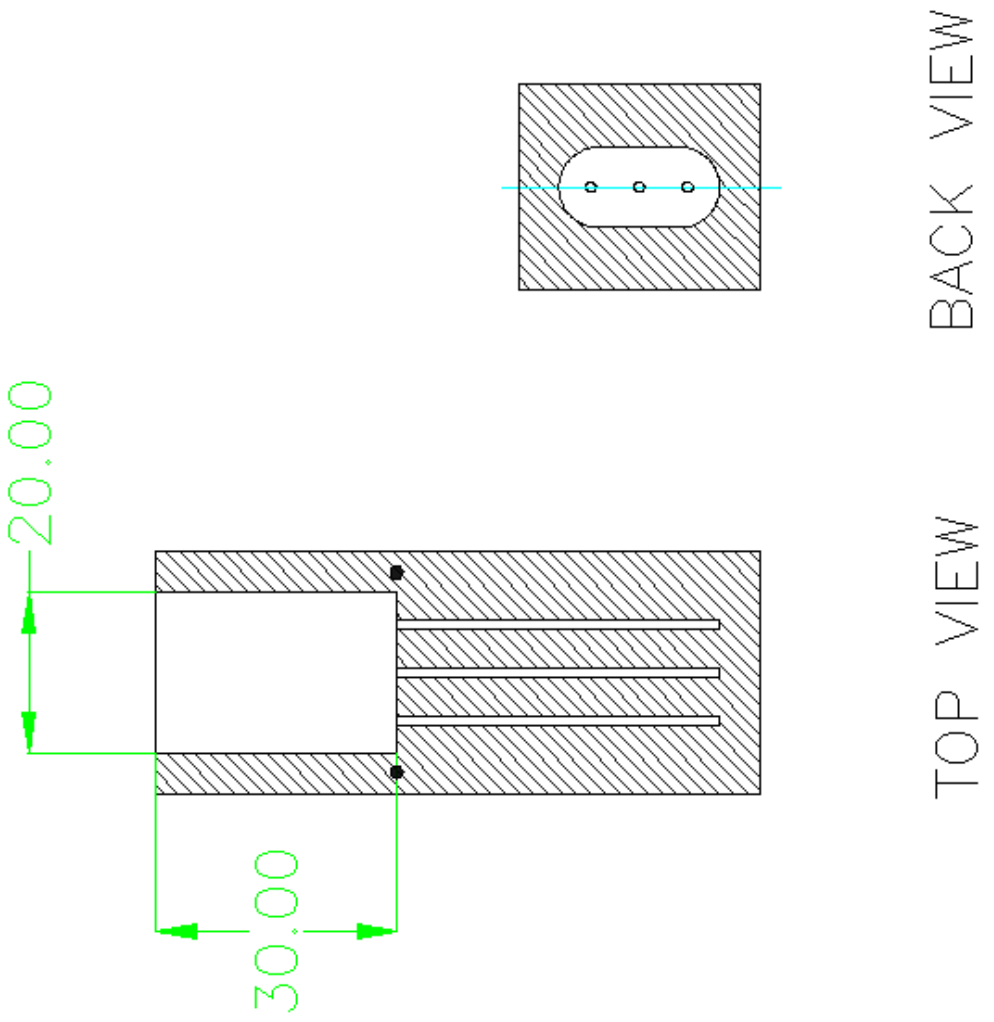

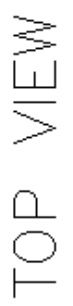

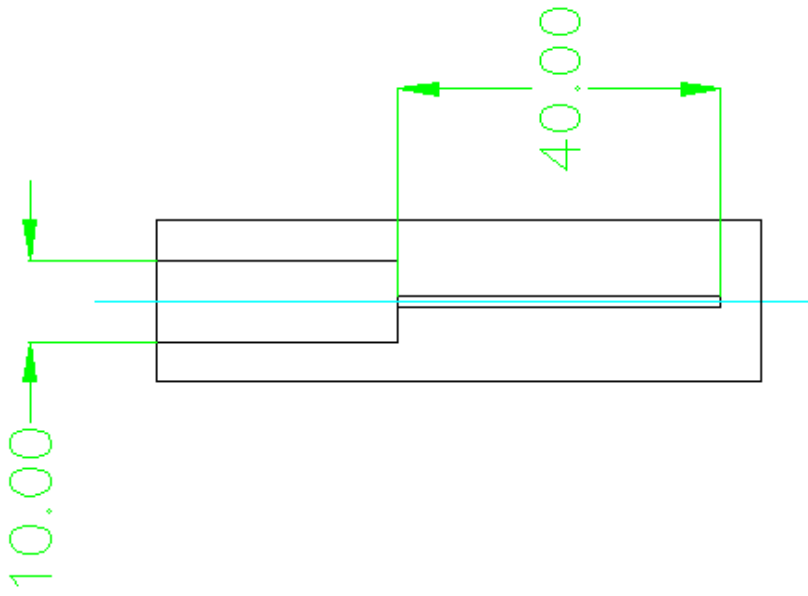

$\exists$
$\bar{\square}$
$\square$
$\square$
$\square$ 


\section{B.2 Aluminum Alloy 2024 Plate}

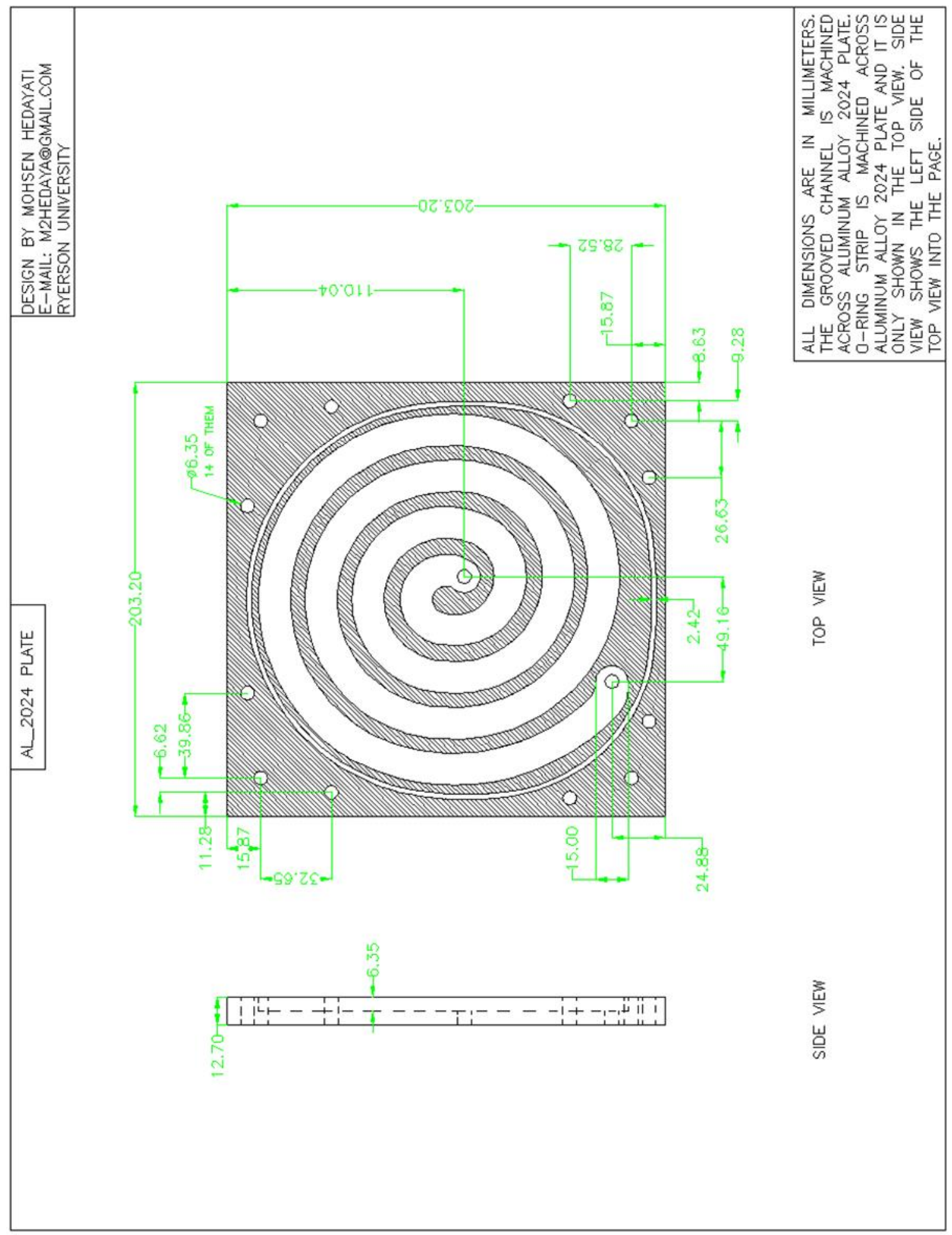




\section{B.3 Stainless Steel Inner Cylinder for Modified Model \#2}

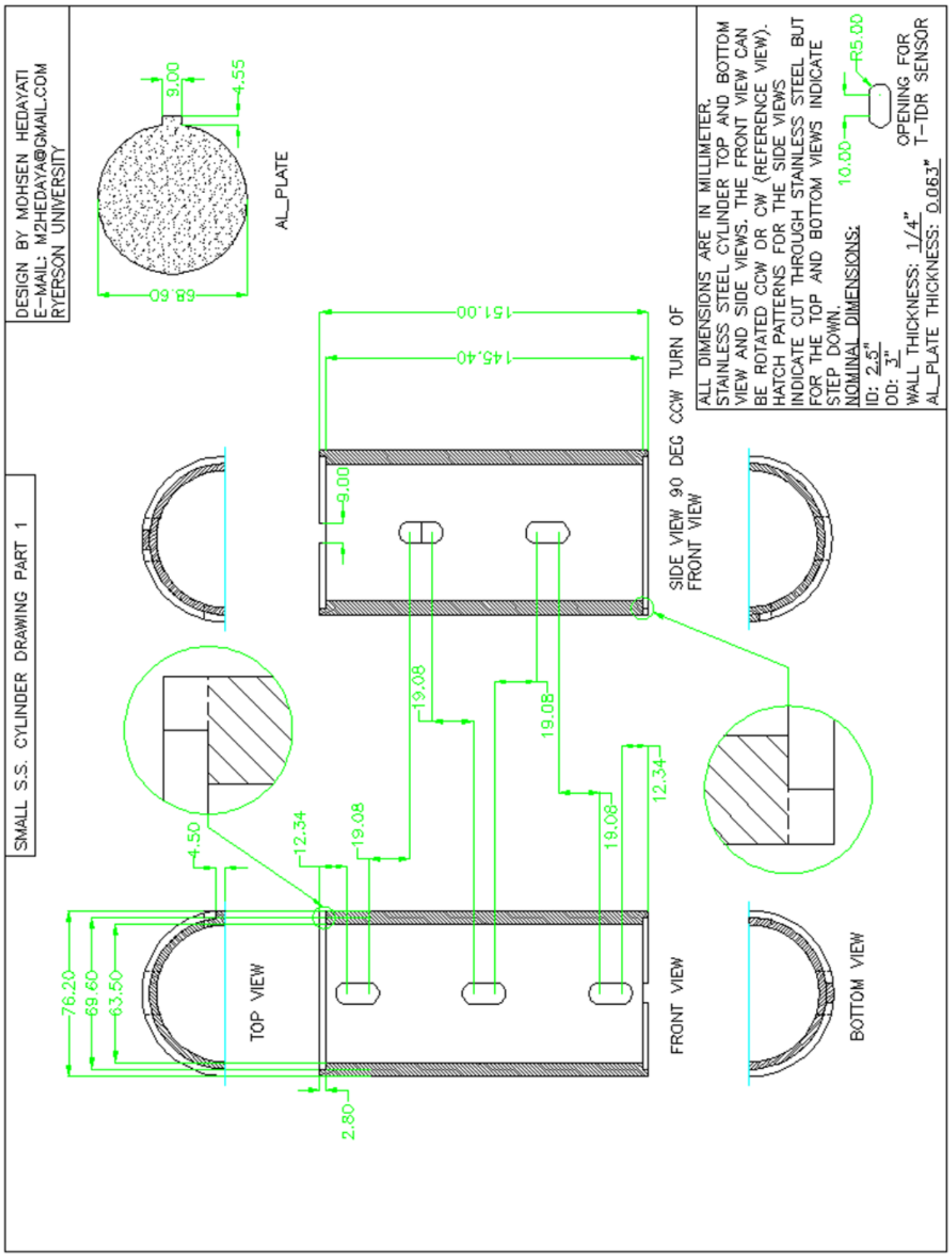




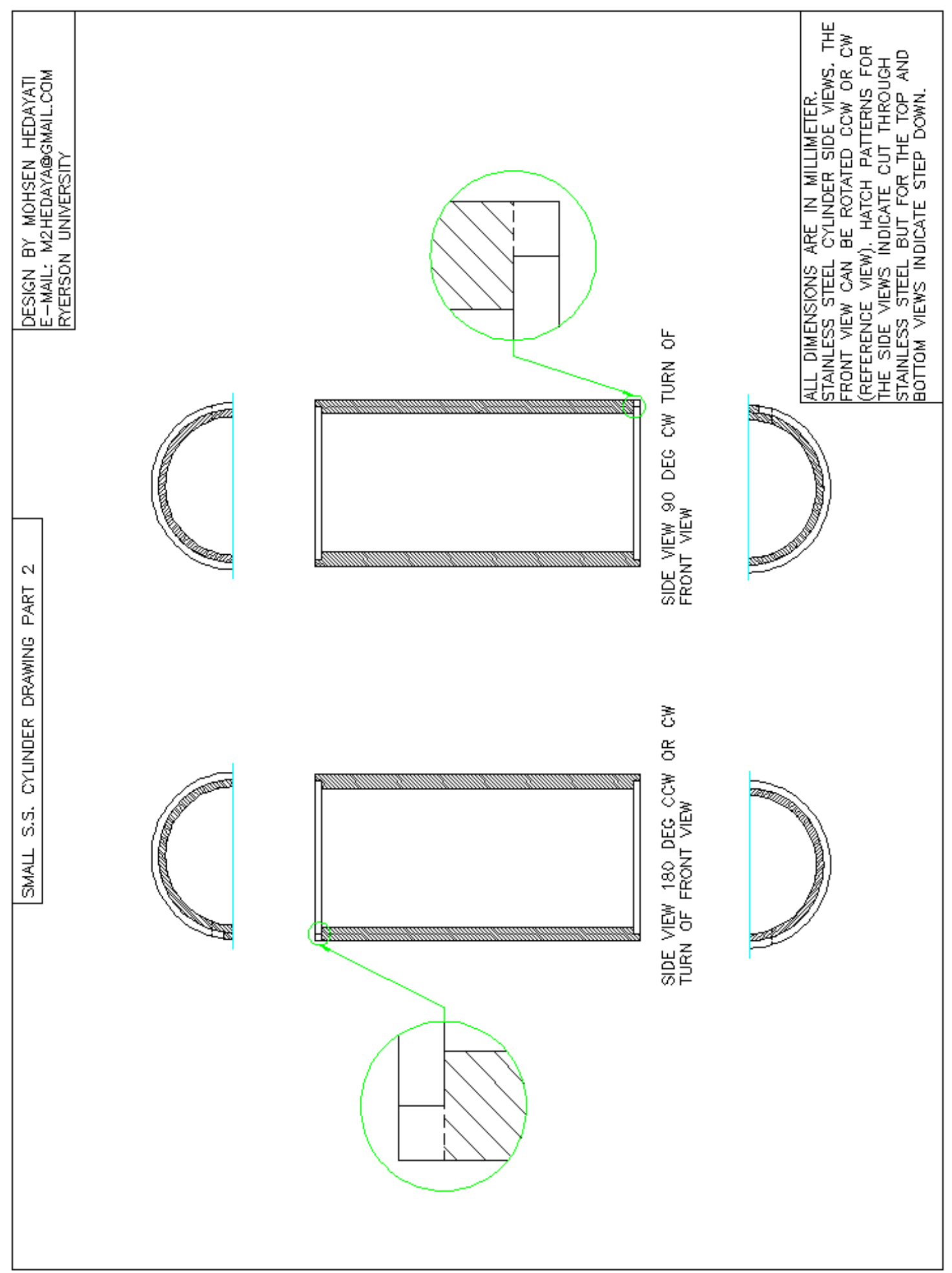




\section{B.4 Stainless Steel Outer Cylinder}

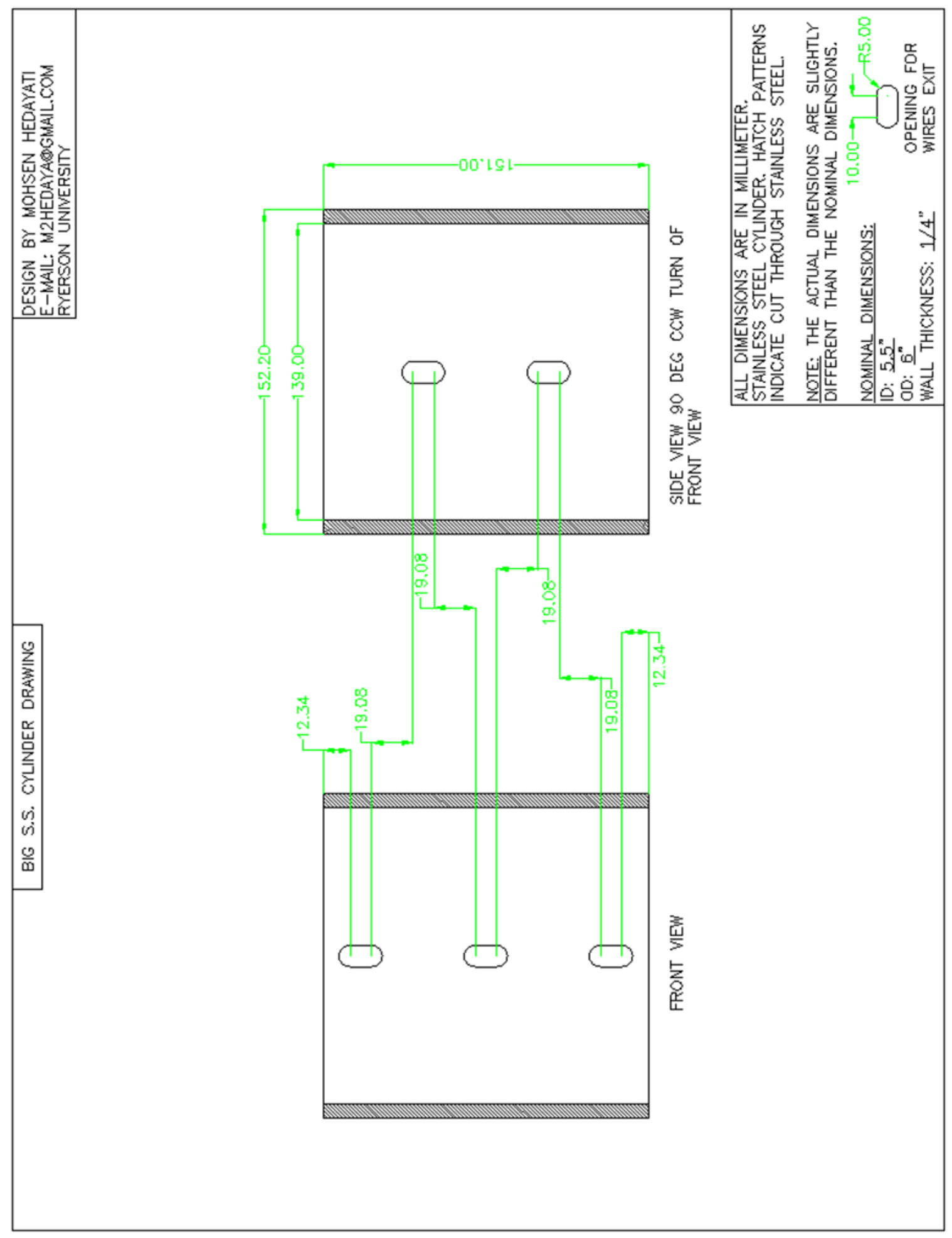




\section{B.5 Stainless Steel Inner Cylinder for Modified Model \#3}

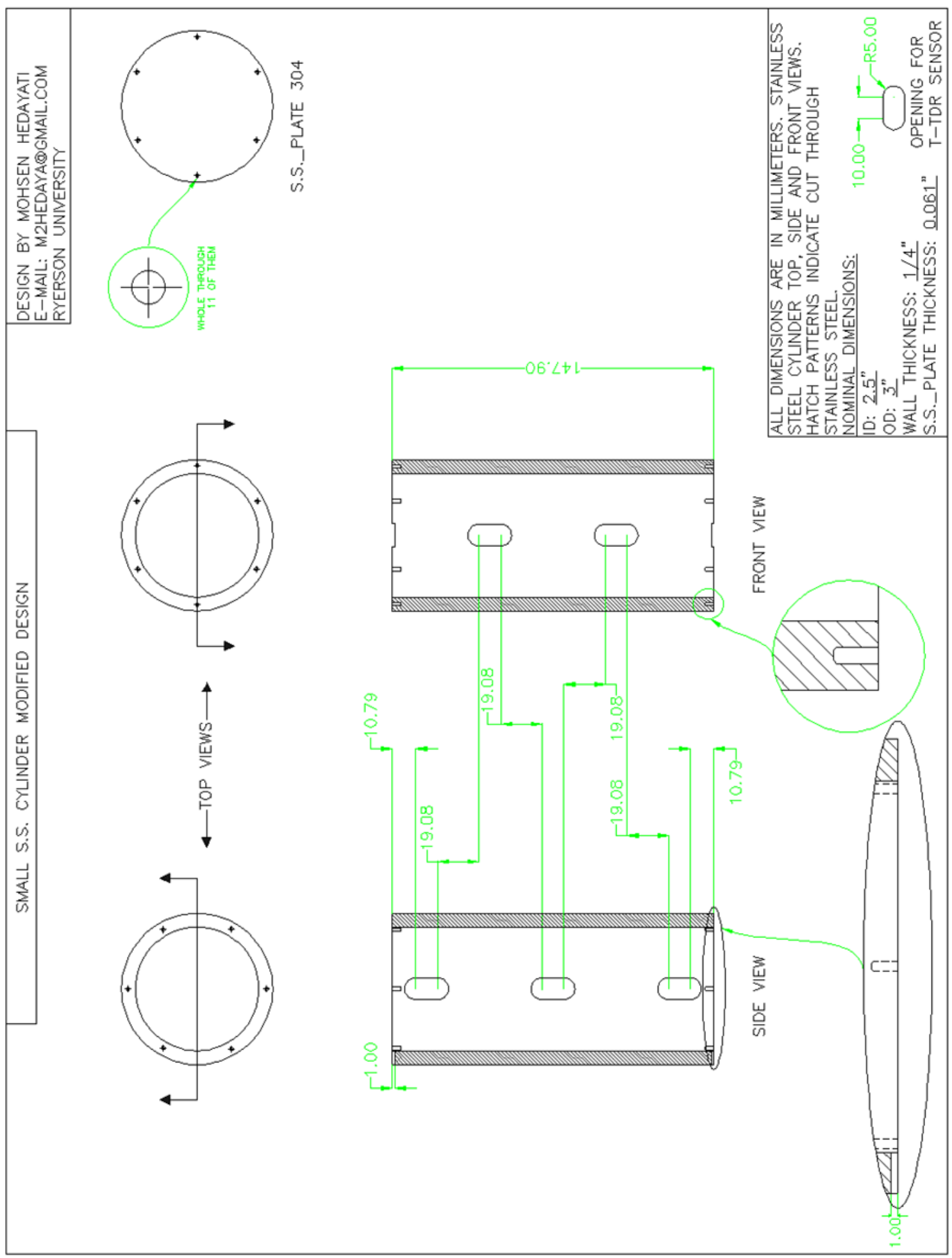




\section{B.6 Bolts, Nuts and Pipe connectors}

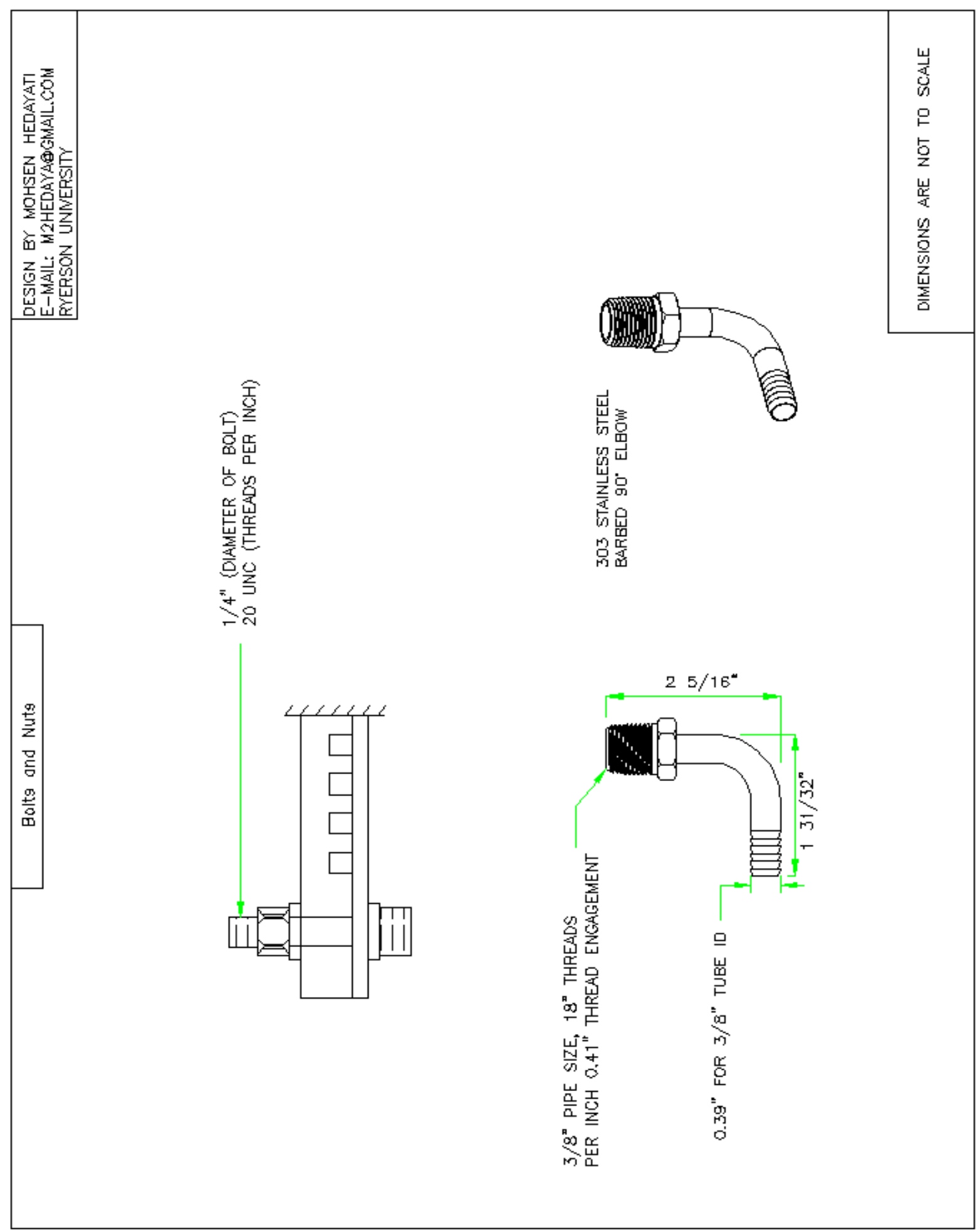




\section{APPENDIX C}

\section{EXPERIMENTAL PHOTOSHOOTS}

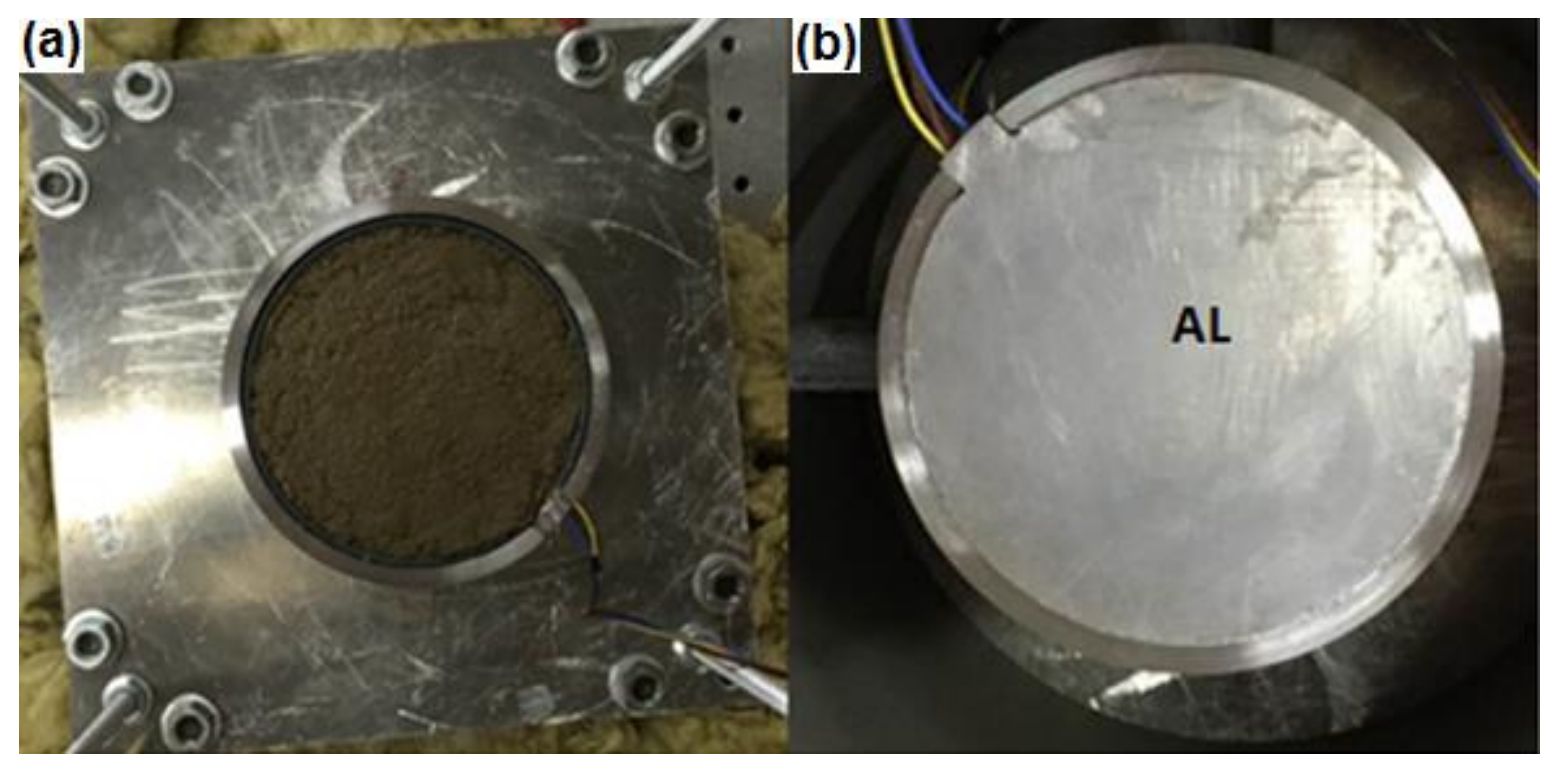

Fig. C.1 Soil cell of modified model \#2 containing wet soil: (a) Soil cell without the top aluminum cap (b) Soil cell with the top aluminum cap

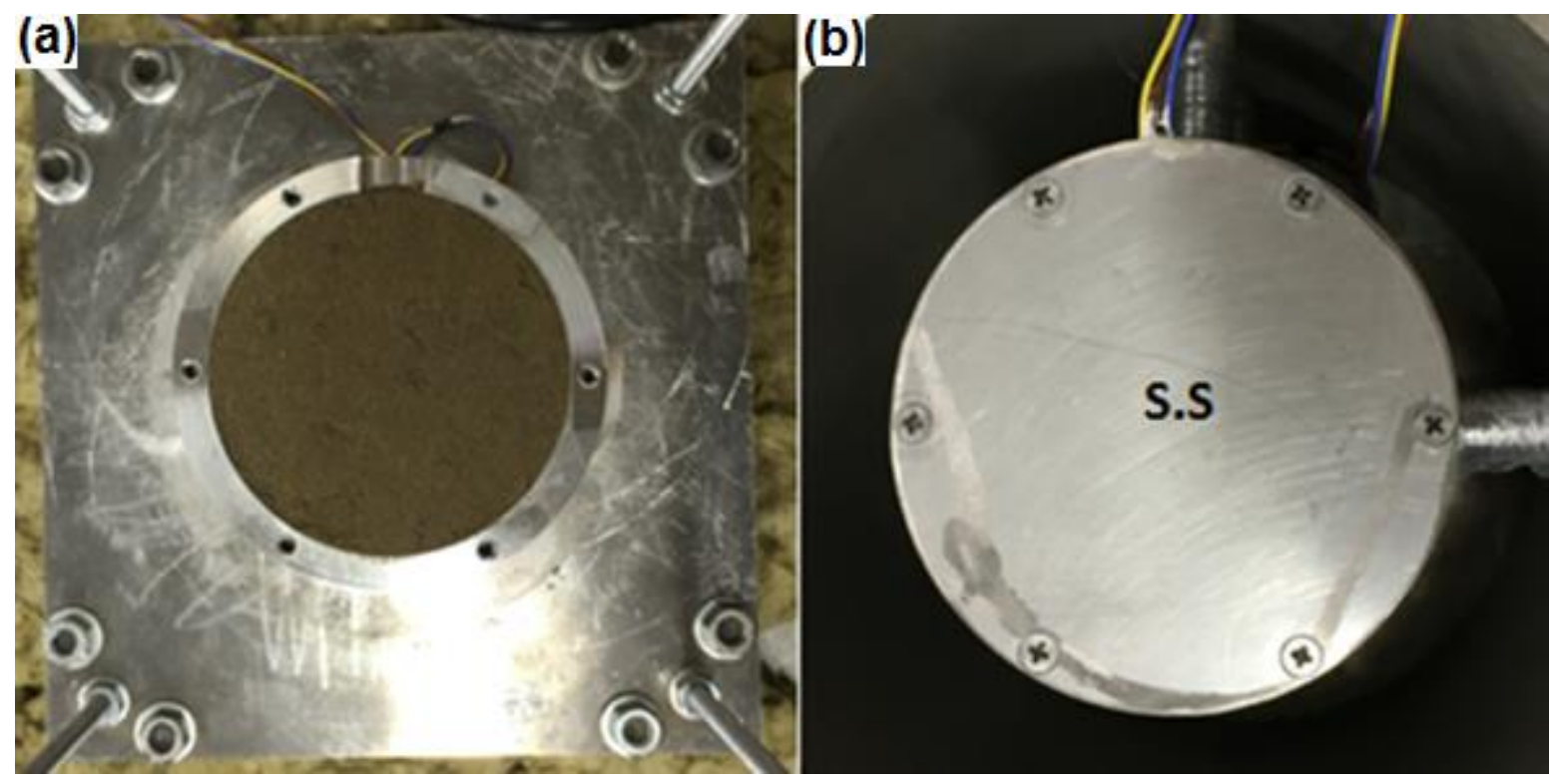

Fig. C.2 Soil cell of modified model \#3 containing wet soil: (a) Soil cell without the top stainless steel cap (b) Soil cell with the top stainless steel cap 


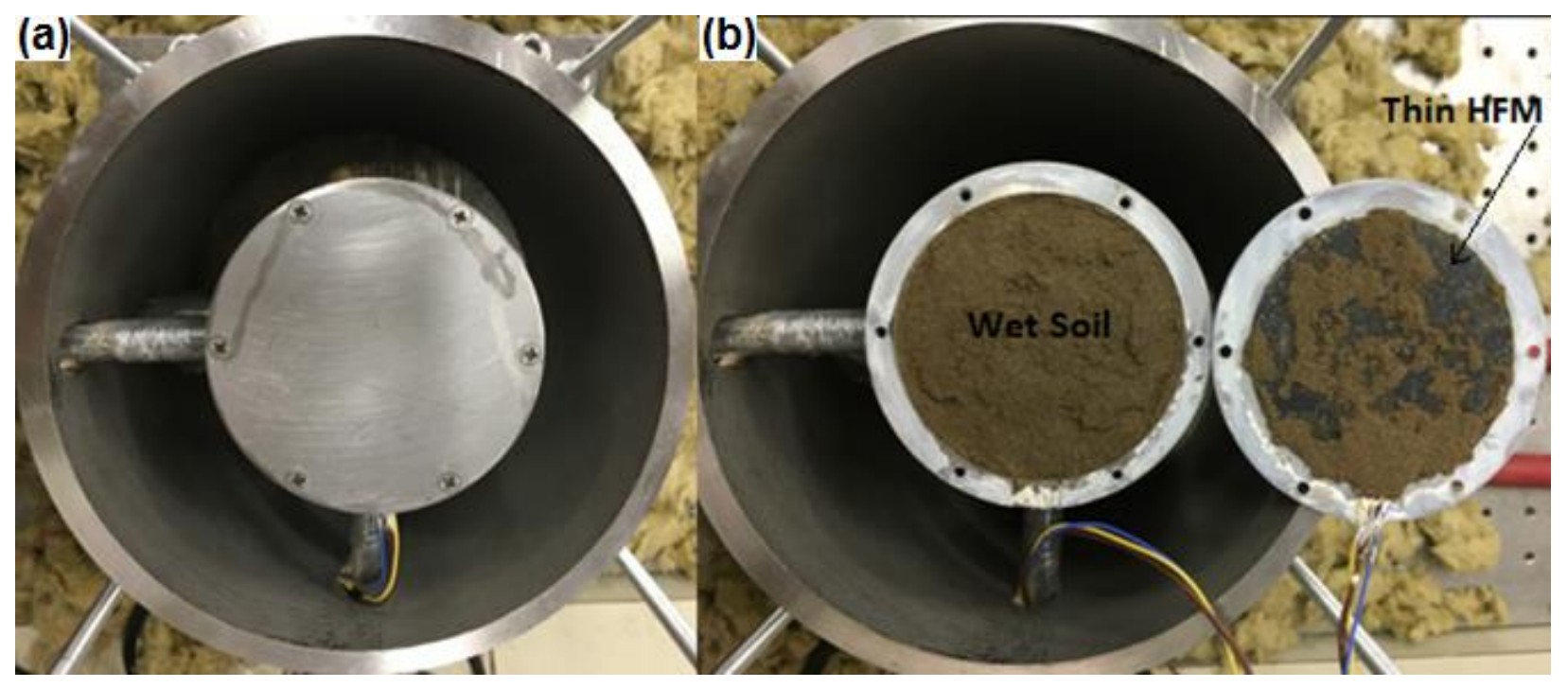

Fig. C.3 From left to right: (a) Top view of the closed cap soil cell of modified model \#3, containing wet soil, with inserted in-house made T-TDR probes which is surrounded by outer stainless steel tube, (b) Top view of the opened cap soil cell of modified model \#3, containing wet soil, with inserted in-house made T-TDR probes which is surrounded by outer stainless steel tube (as shown, thin HFM is attached to the surface of the thin stainless steel plate)

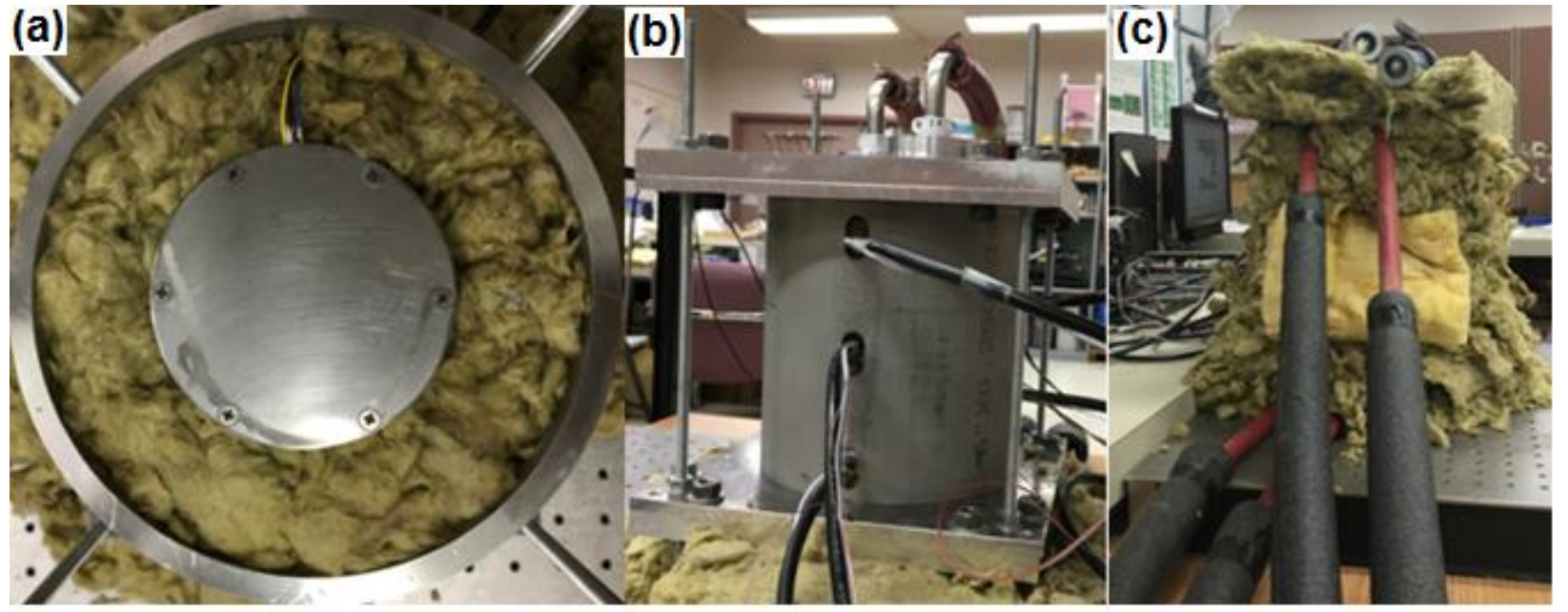

Fig. C.4 From left to right: (a) Top view of modified model \#3 soil cell which is surrounded by fiber glass insulation and outer stainless steel tube, (b) Side view of the apparatus without outer insulation layer and (c) Side view of the apparatus with outer insulation layer 


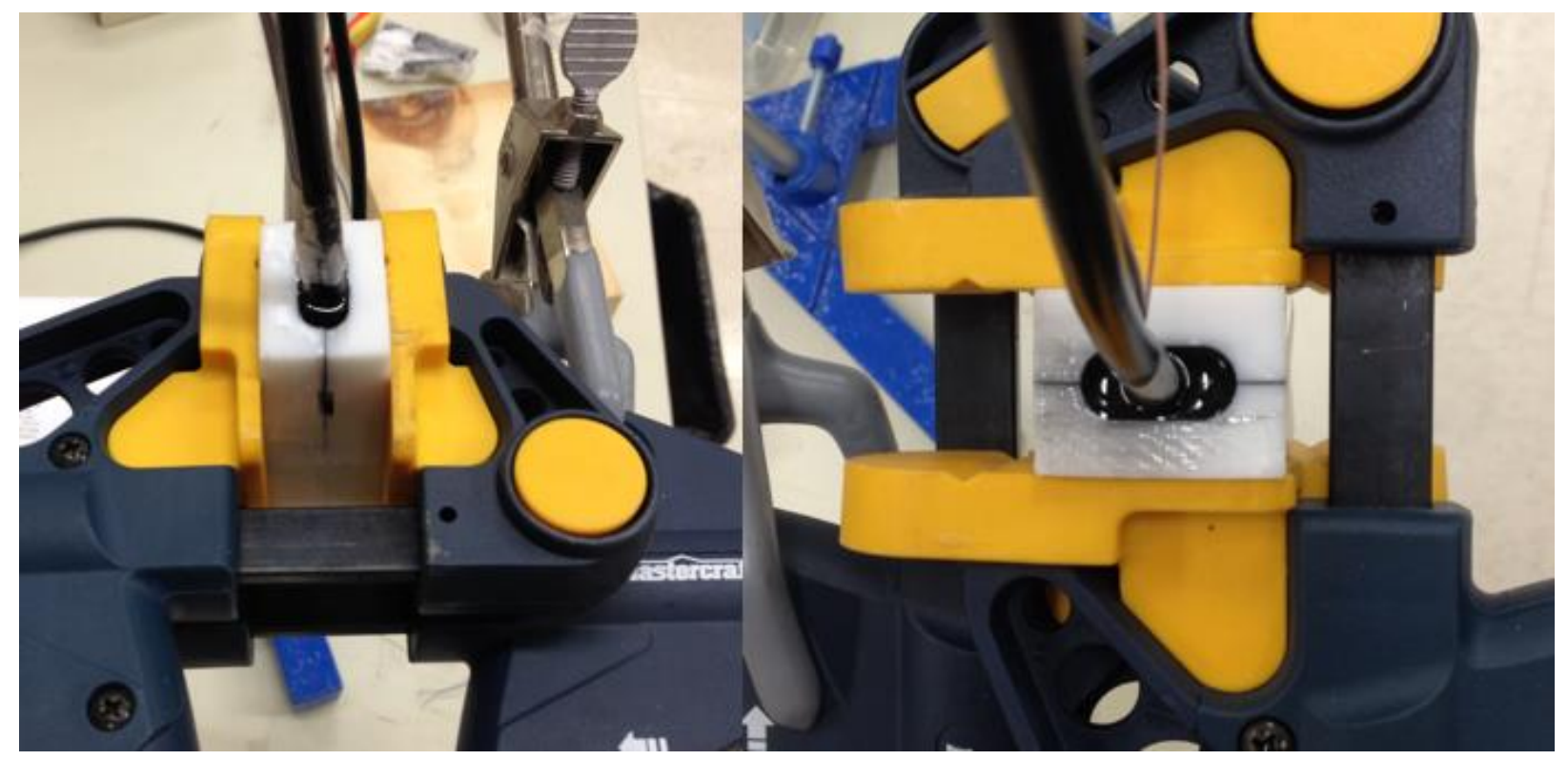

Fig. C.5 T-TDR probe mold casting clamped on the support bar

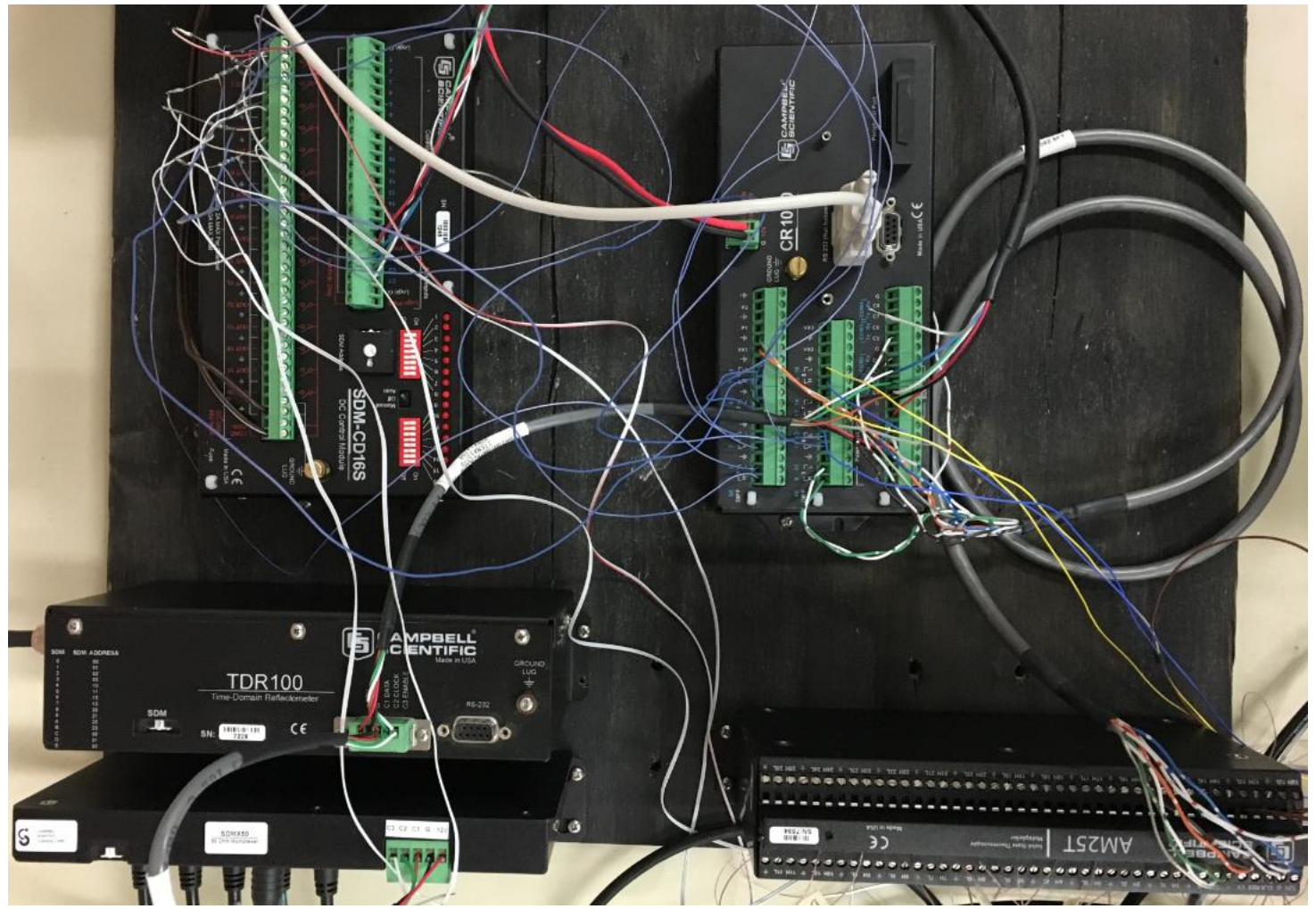

Fig. C.6 Campbell Scientific digital system includes SDMS-CD16S (relay controller), Data Logger (CR-1000), TDR-100 and AM25T (multiplexer for the thermocouples) 


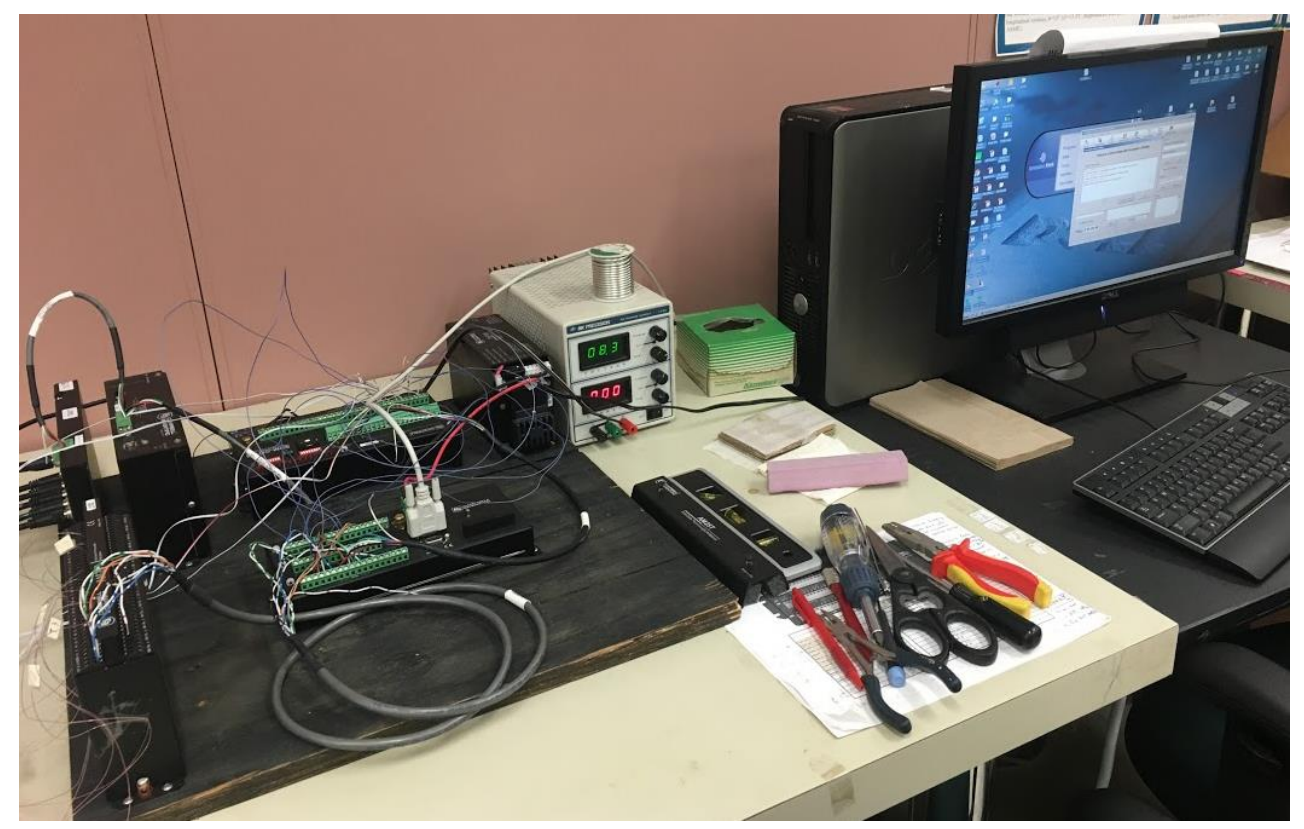

Fig. C.7 Campbell Scientific digital system and DC power supply connected to the PC via 8 conductor cable from Data Logger (CR-1000)

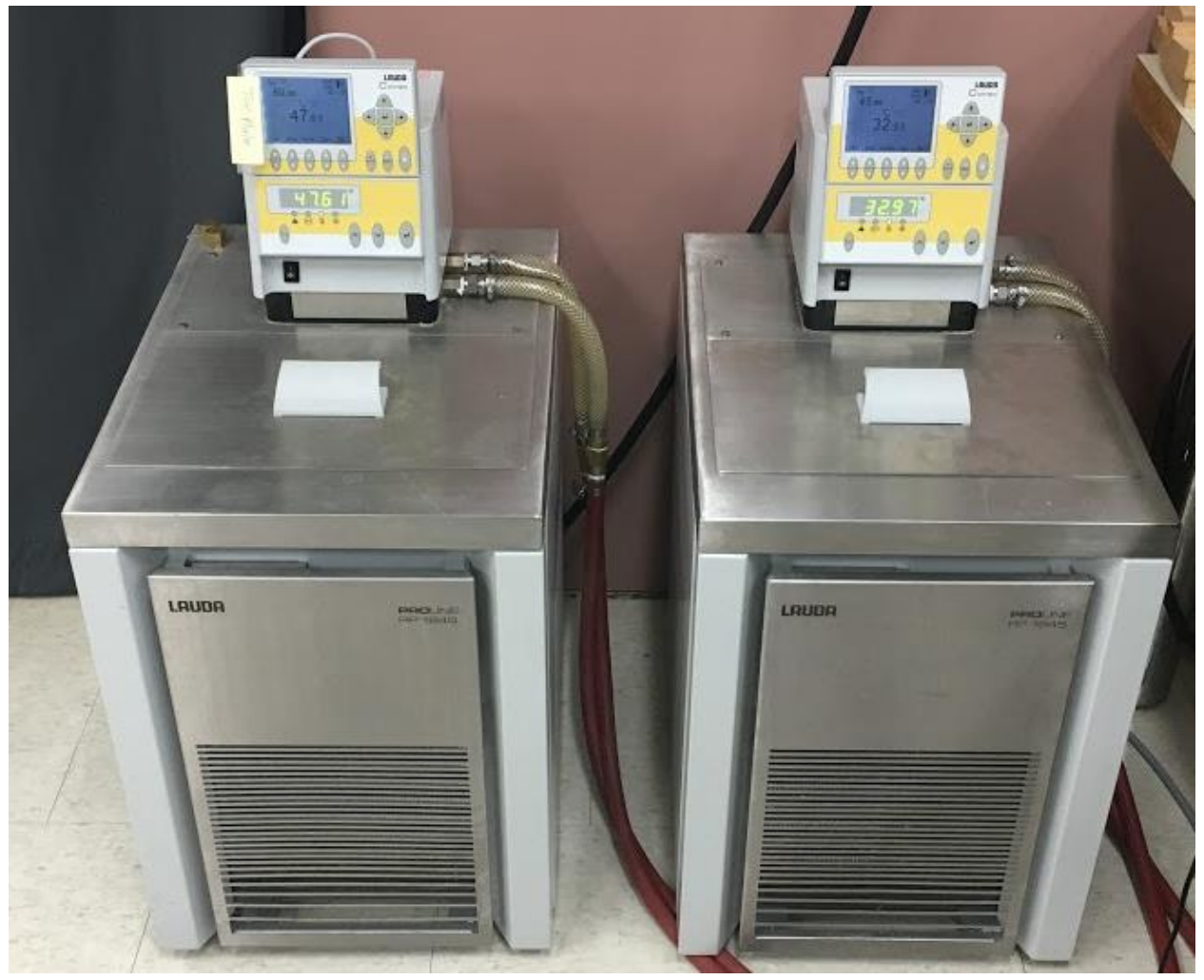

Fig. C.8 Lauda Proline RP1845 Thermal Baths 


\section{APPENDIX D}

\section{EXPERIMENTAL RESULTS OF THE WET SOIL}

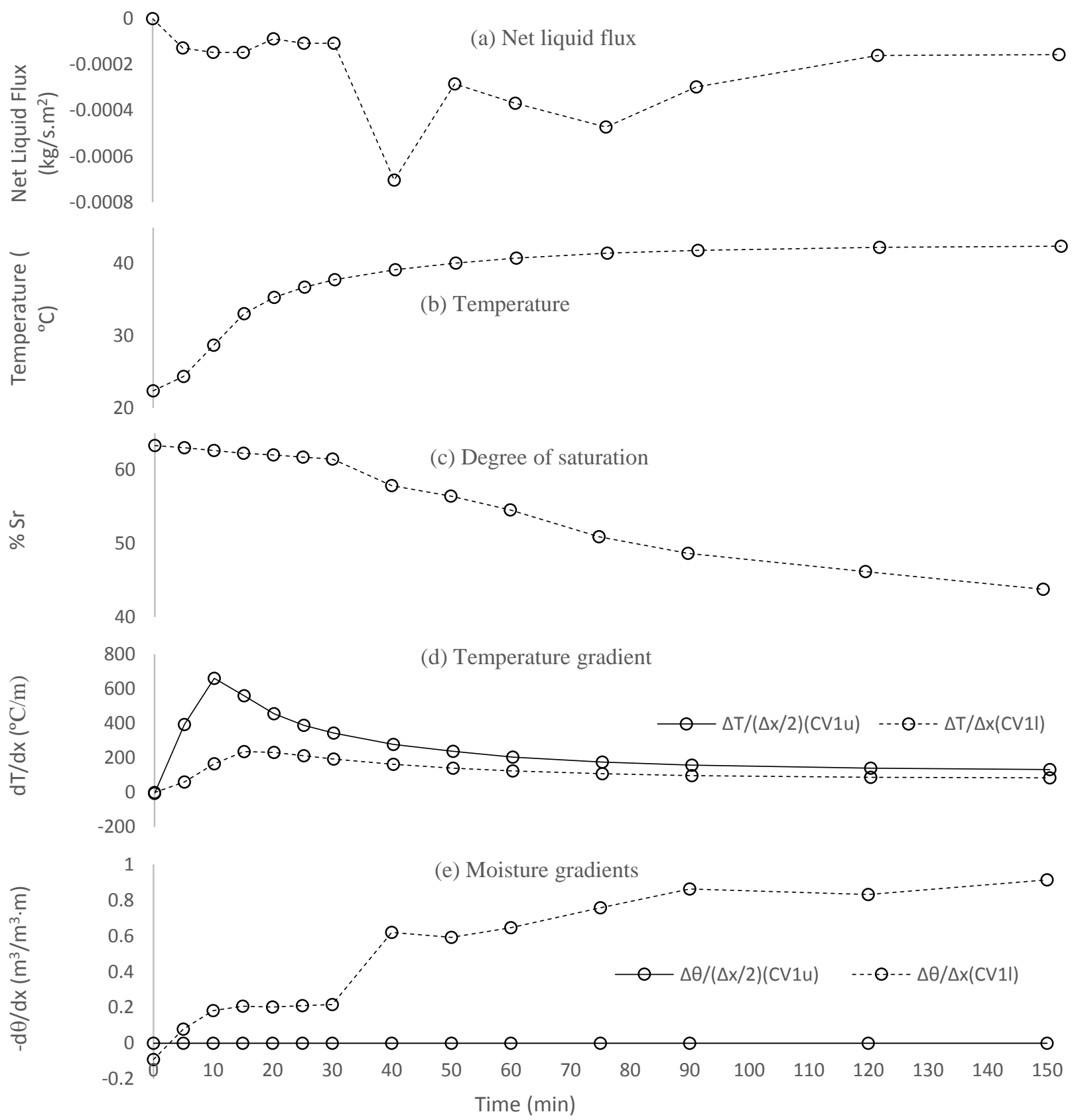

Fig. D.1 The experimental results for control volume 1 of the soil column for the case of $45^{\circ} \mathrm{C}$ hot plate and $30^{\circ} \mathrm{C}$ cold plate 

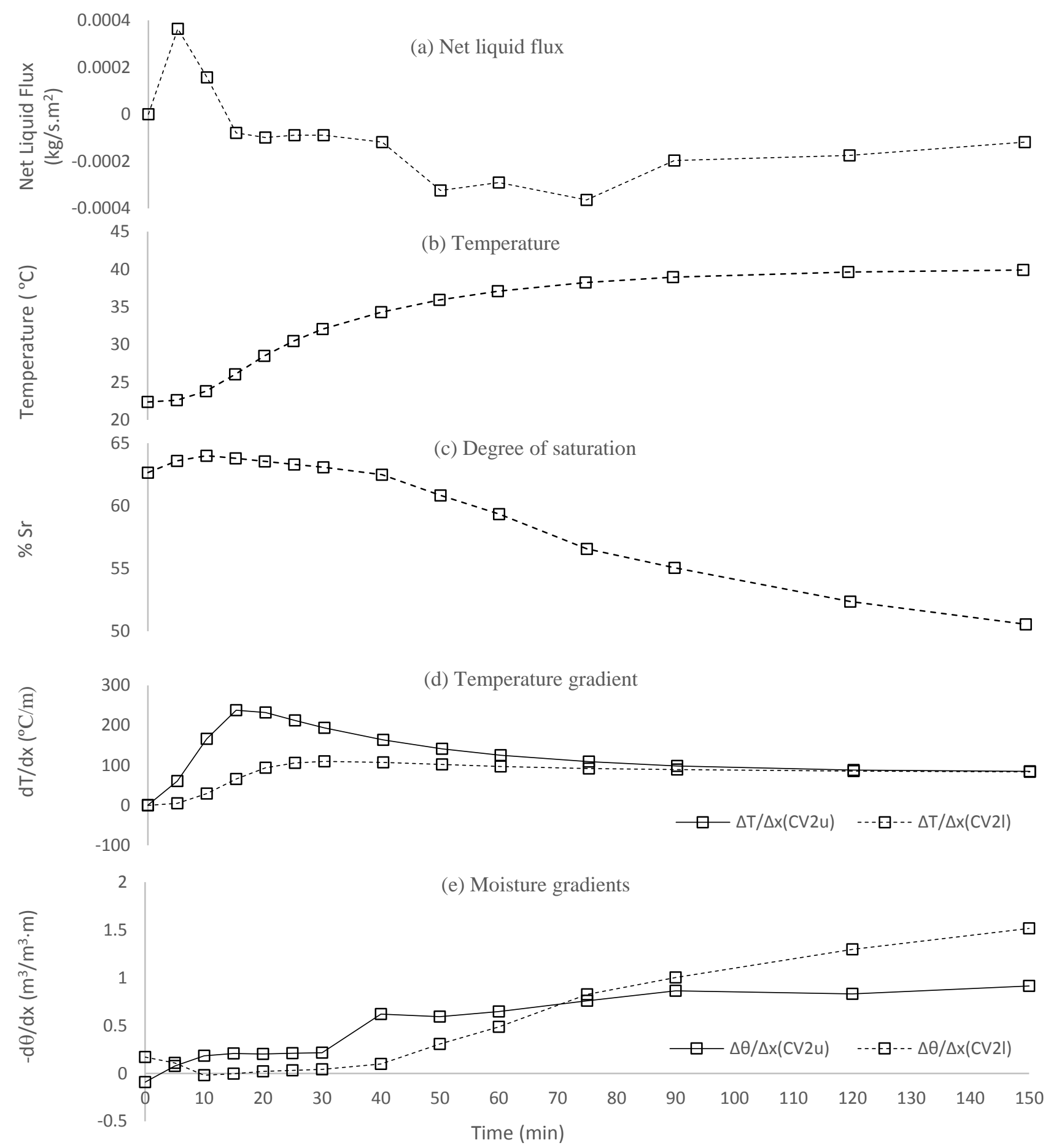

Fig. D.2 The experimental results for control volume 2 of the soil column for the case of $45^{\circ} \mathrm{C}$ hot plate and $30^{\circ} \mathrm{C}$ cold plate 


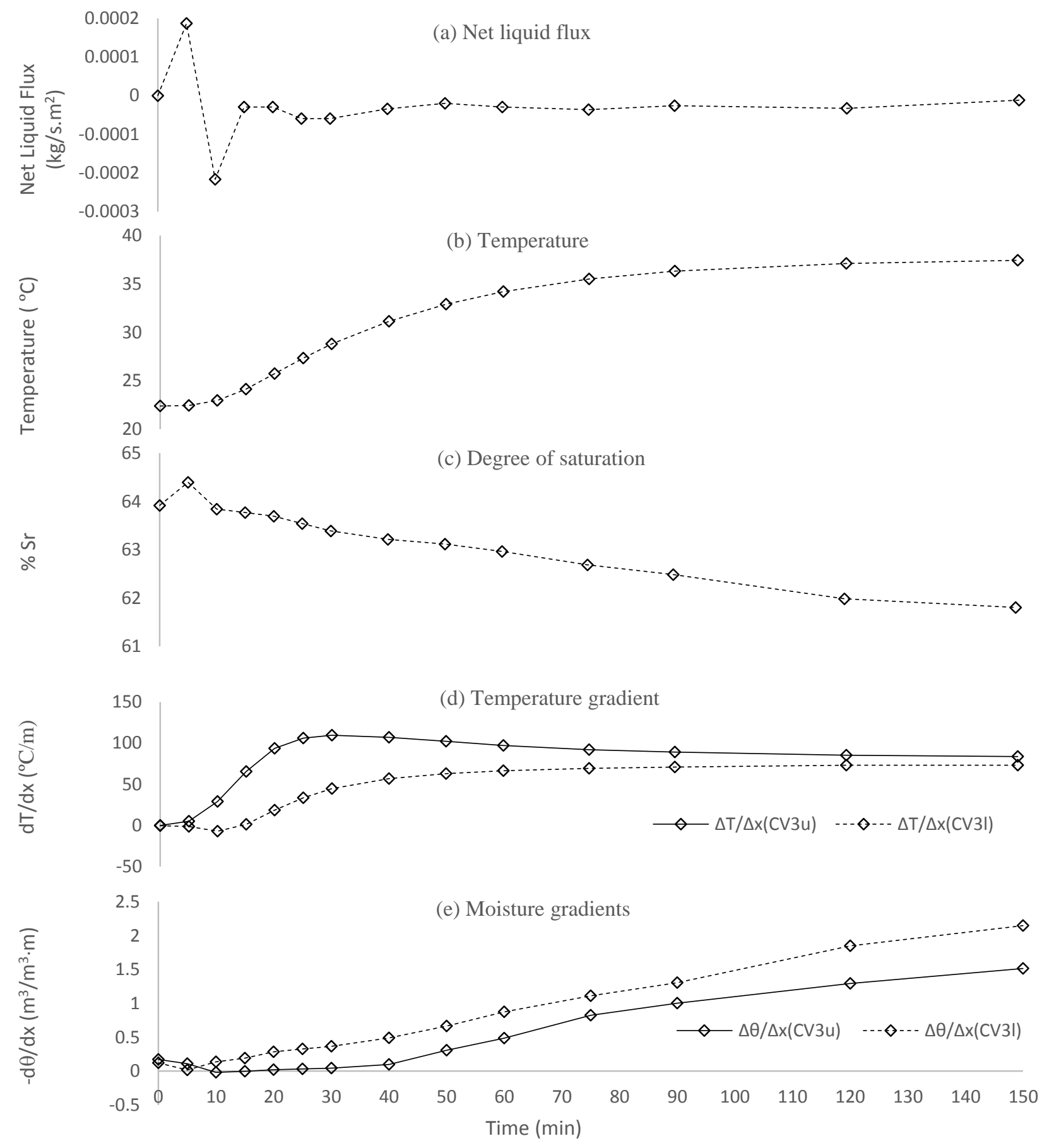

Fig. D.3 The experimental results for control volume 3 of the soil column for the case of $45^{\circ} \mathrm{C}$ hot plate and $30^{\circ} \mathrm{C}$ cold plate 

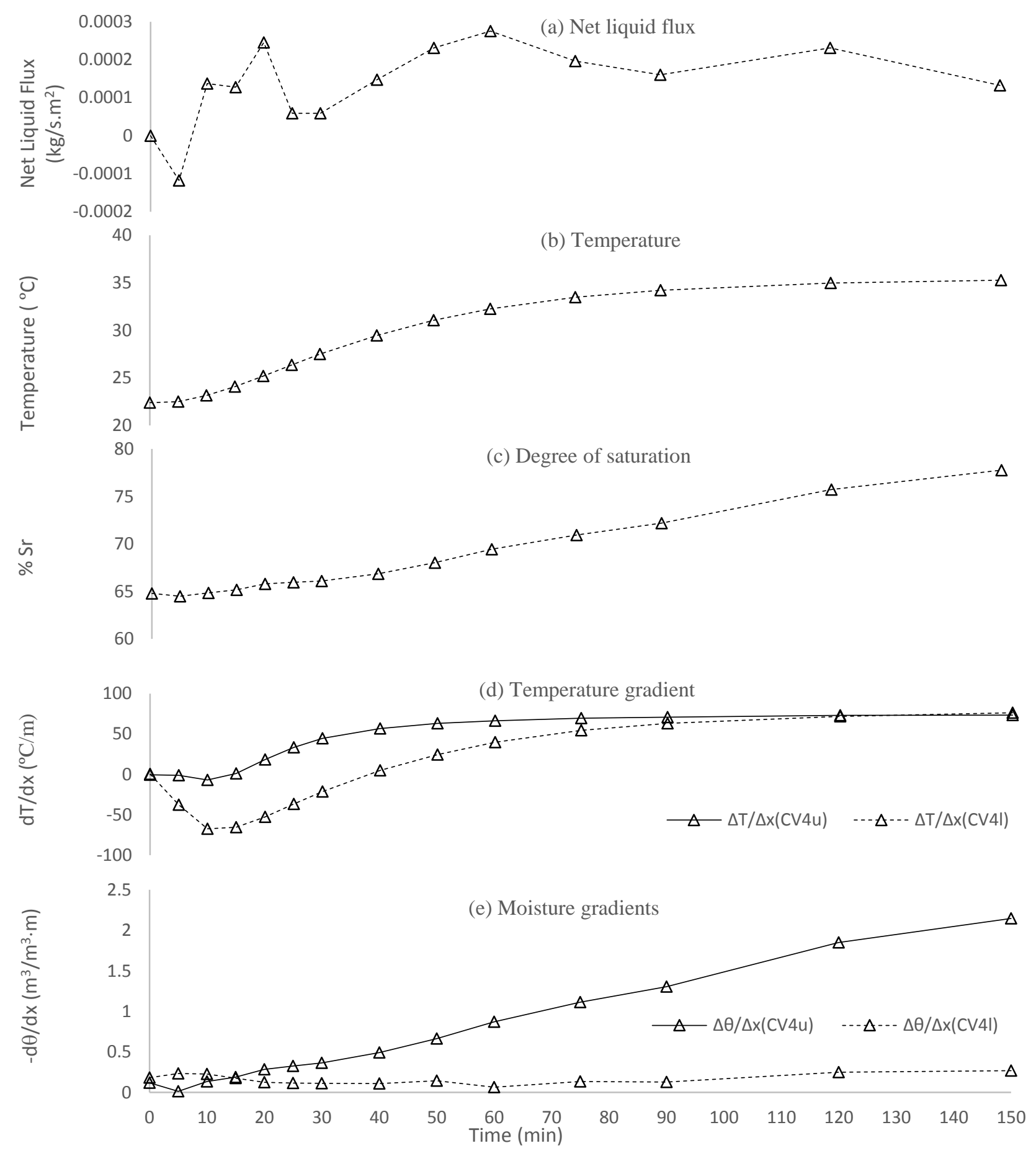

Fig. D.4 The experimental results for control volume 4 of the soil column for the case of $45^{\circ} \mathrm{C}$ hot plate and $30^{\circ} \mathrm{C}$ cold plate 

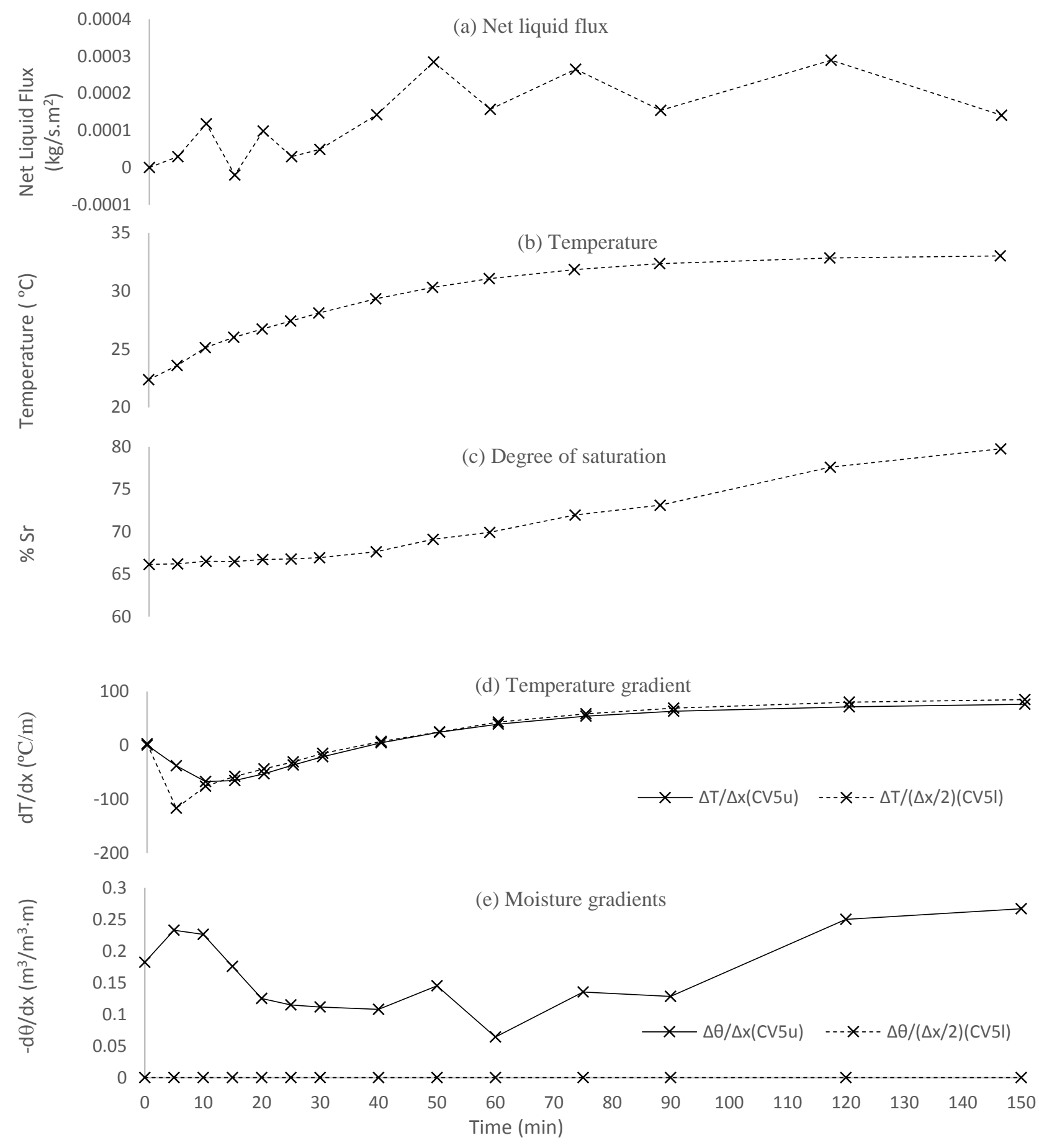

Fig. D.5 The experimental results for control volume 5 of the soil column for the case of $45^{\circ} \mathrm{C}$ hot plate and $30^{\circ} \mathrm{C}$ cold plate 

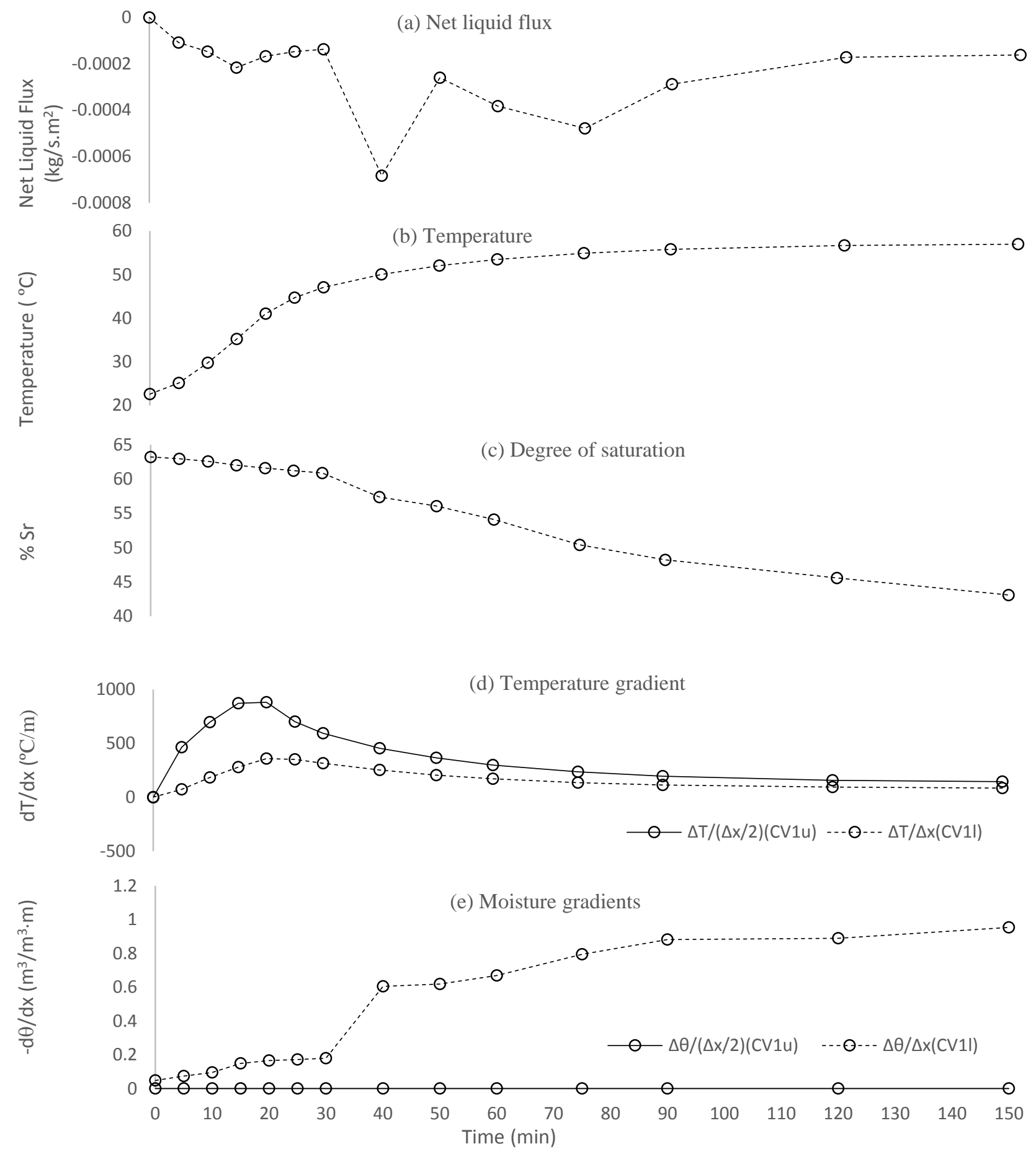

Fig. D.6 The experimental results for control volume 1 of the soil column for the case of $60^{\circ} \mathrm{C}$ hot plate and $45^{\circ} \mathrm{C}$ cold plate 

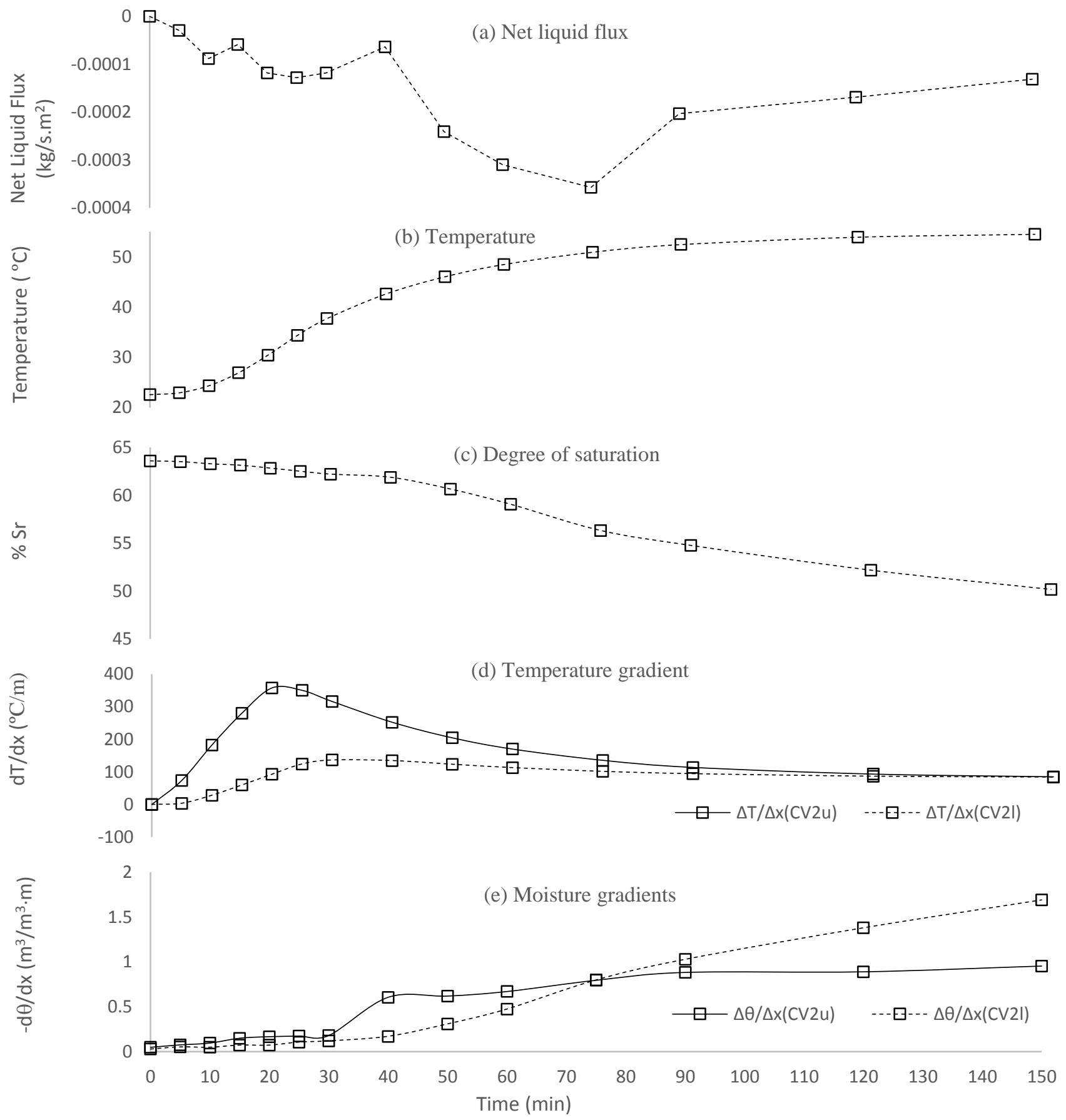

Fig. D.7 The experimental results for control volume 2 of the soil column for the case of $60^{\circ} \mathrm{C}$ hot plate and $45^{\circ} \mathrm{C}$ cold plate 

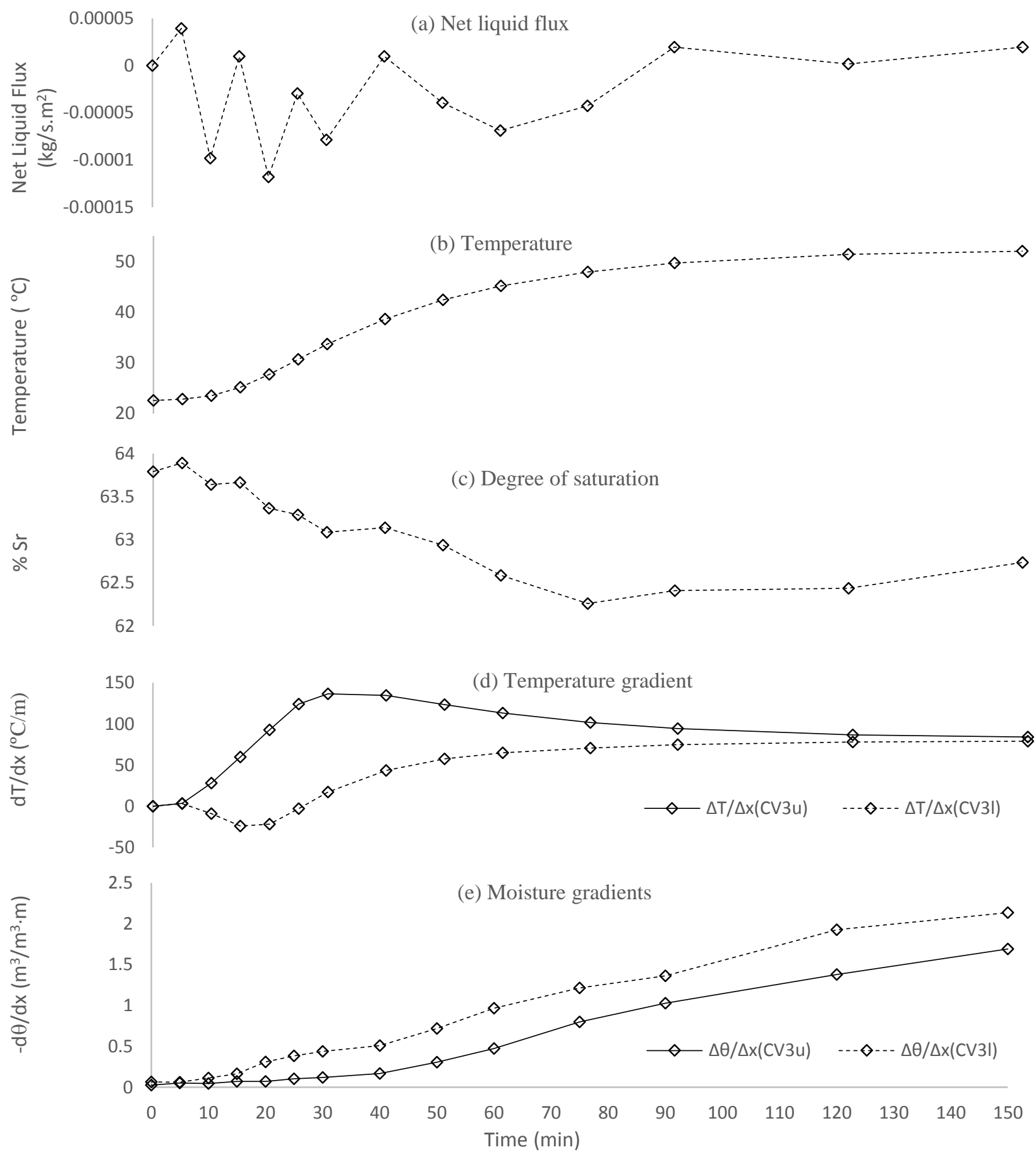

Fig. D.8 The experimental results for control volume 3 of the soil column for the case of $60^{\circ} \mathrm{C}$ hot plate and $45^{\circ} \mathrm{C}$ cold plate 

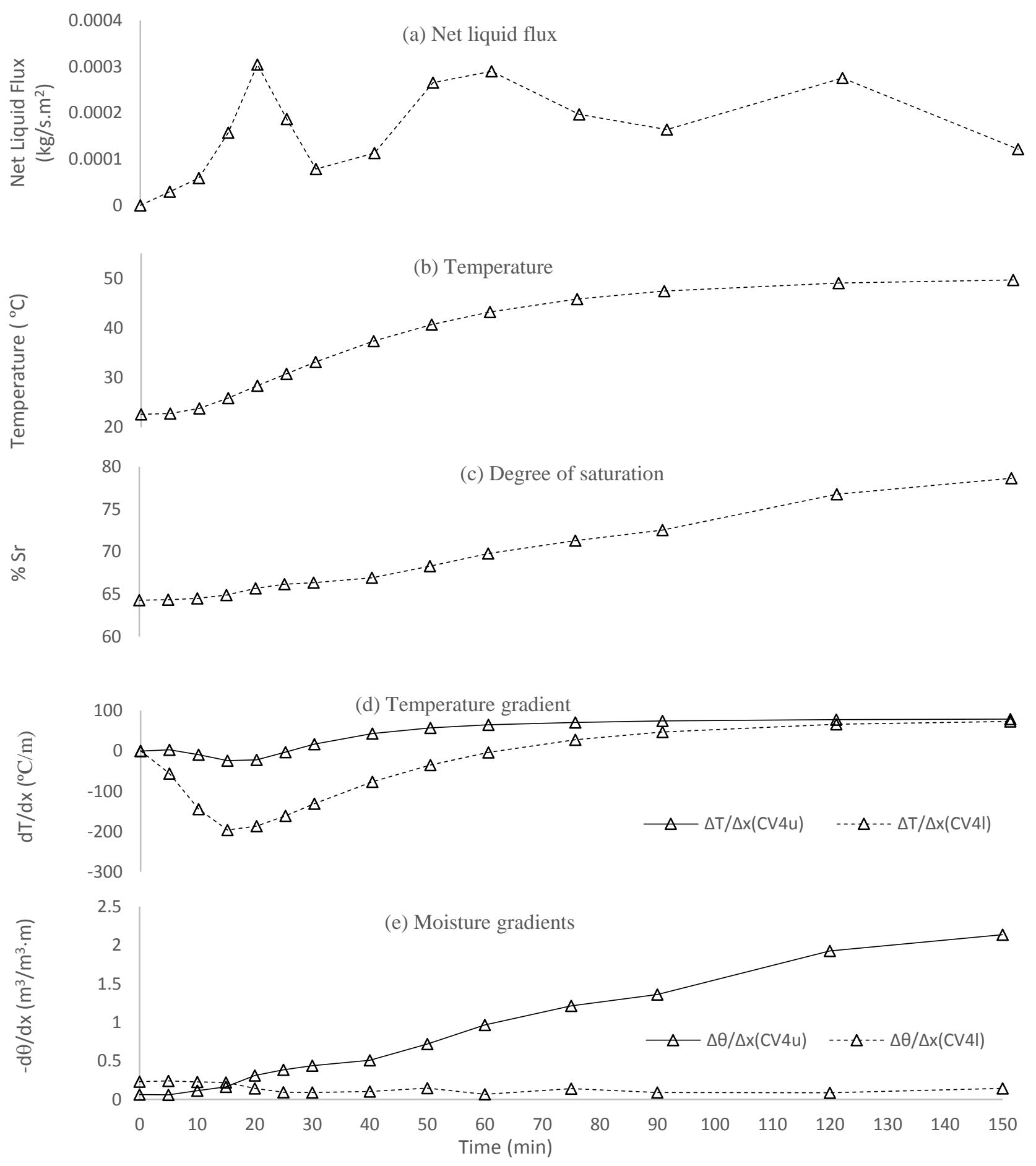

Fig. D.9 The experimental results for control volume 4 of the soil column for the case of $60^{\circ} \mathrm{C}$ hot plate and $45^{\circ} \mathrm{C}$ cold plate 

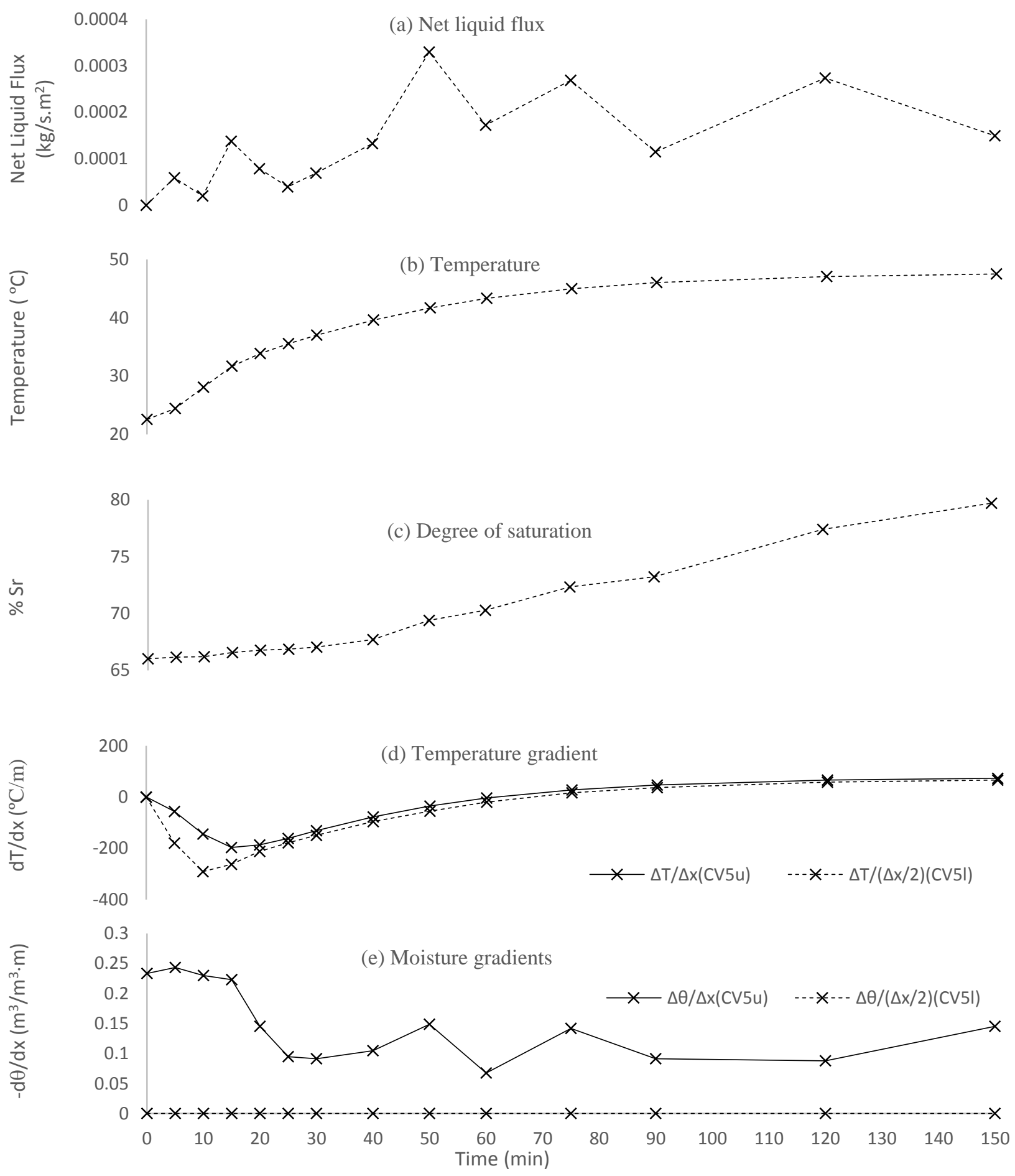

Fig. D.10 The experimental results for control volume 5 of the soil column for the case of $60^{\circ} \mathrm{C}$ hot plate and $45^{\circ} \mathrm{C}$ cold plate 

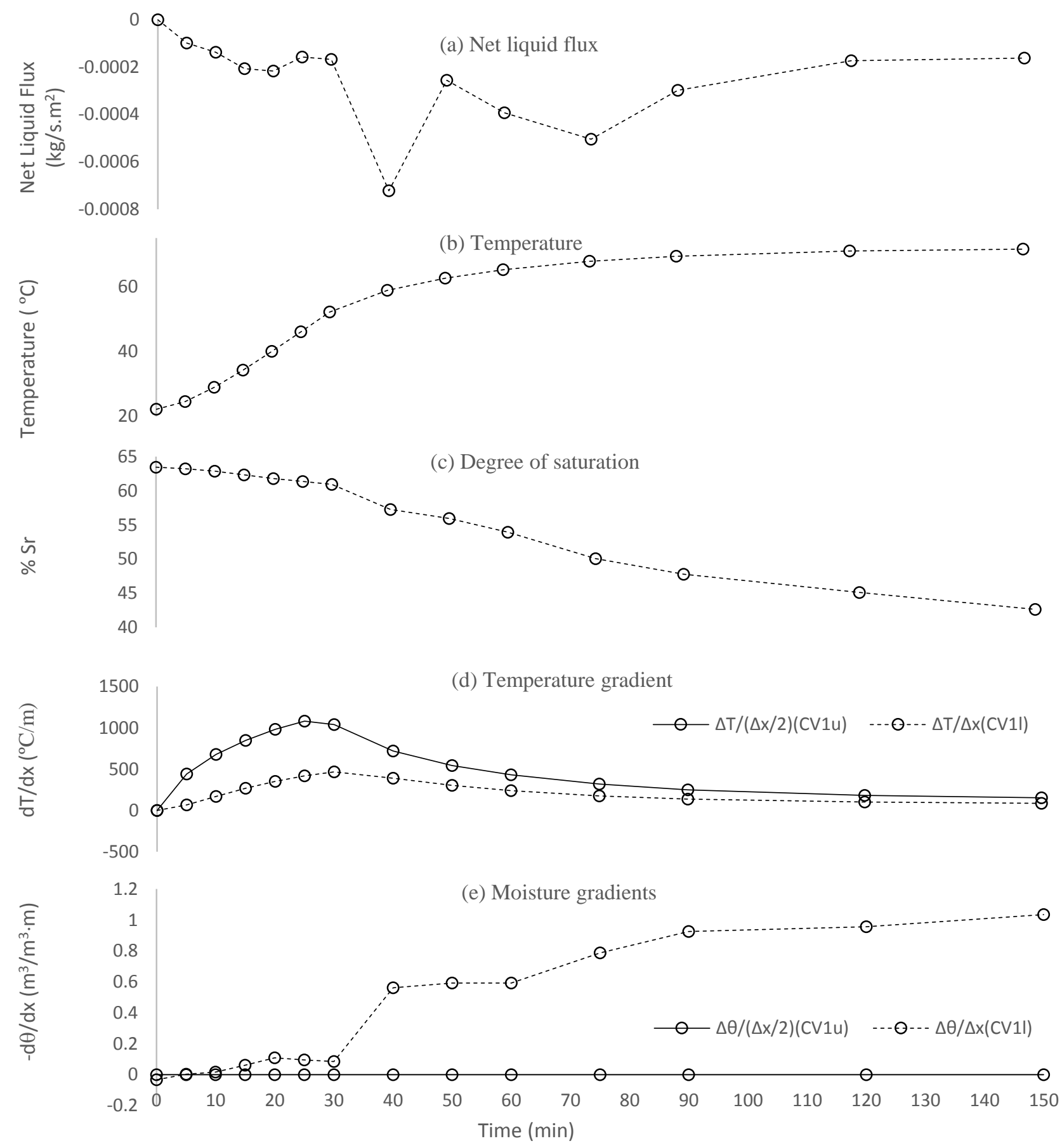

Fig. D.11 The experimental results for control volume 1 of the soil column for the case of $75^{\circ} \mathrm{C}$ hot plate and $60^{\circ} \mathrm{C}$ cold plate 

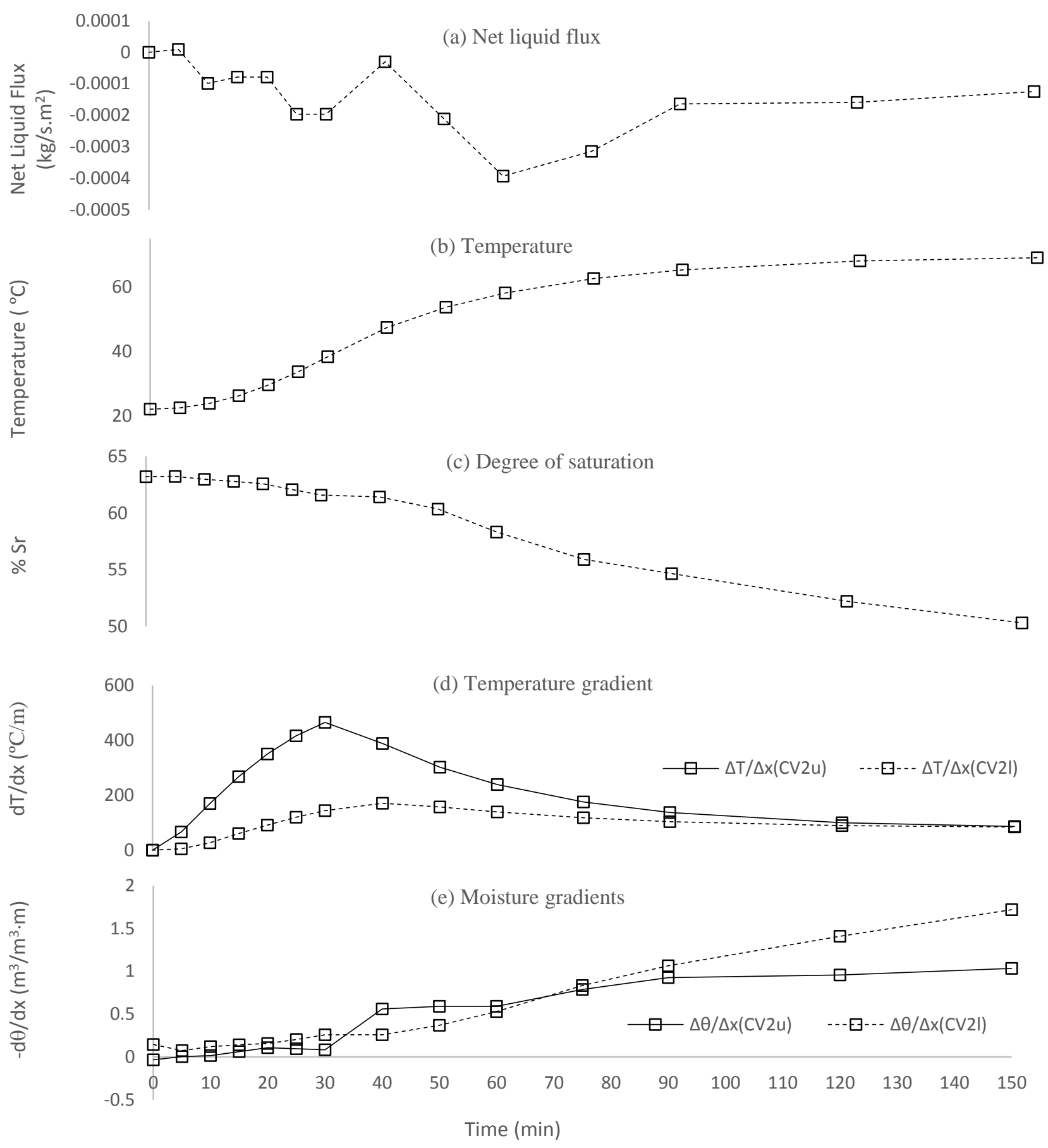

Fig. D.12 The experimental results for control volume 2 of the soil column for the case of $75^{\circ} \mathrm{C}$ hot plate and $60^{\circ} \mathrm{C}$ cold plate 

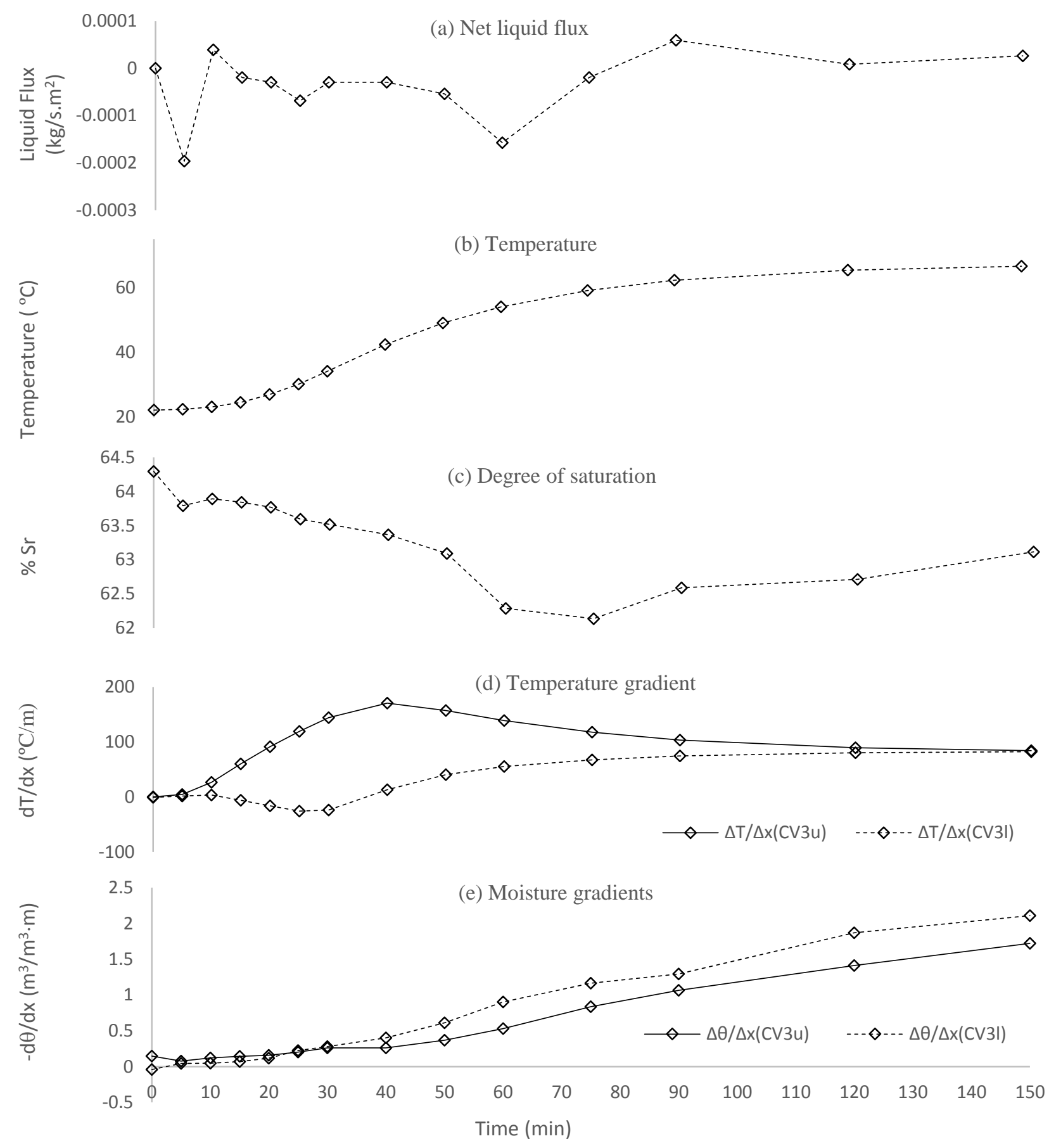

Fig. D.13 The experimental results for control volume 3 of the soil column for the case of $75^{\circ} \mathrm{C}$ hot plate and $60^{\circ} \mathrm{C}$ cold plate 

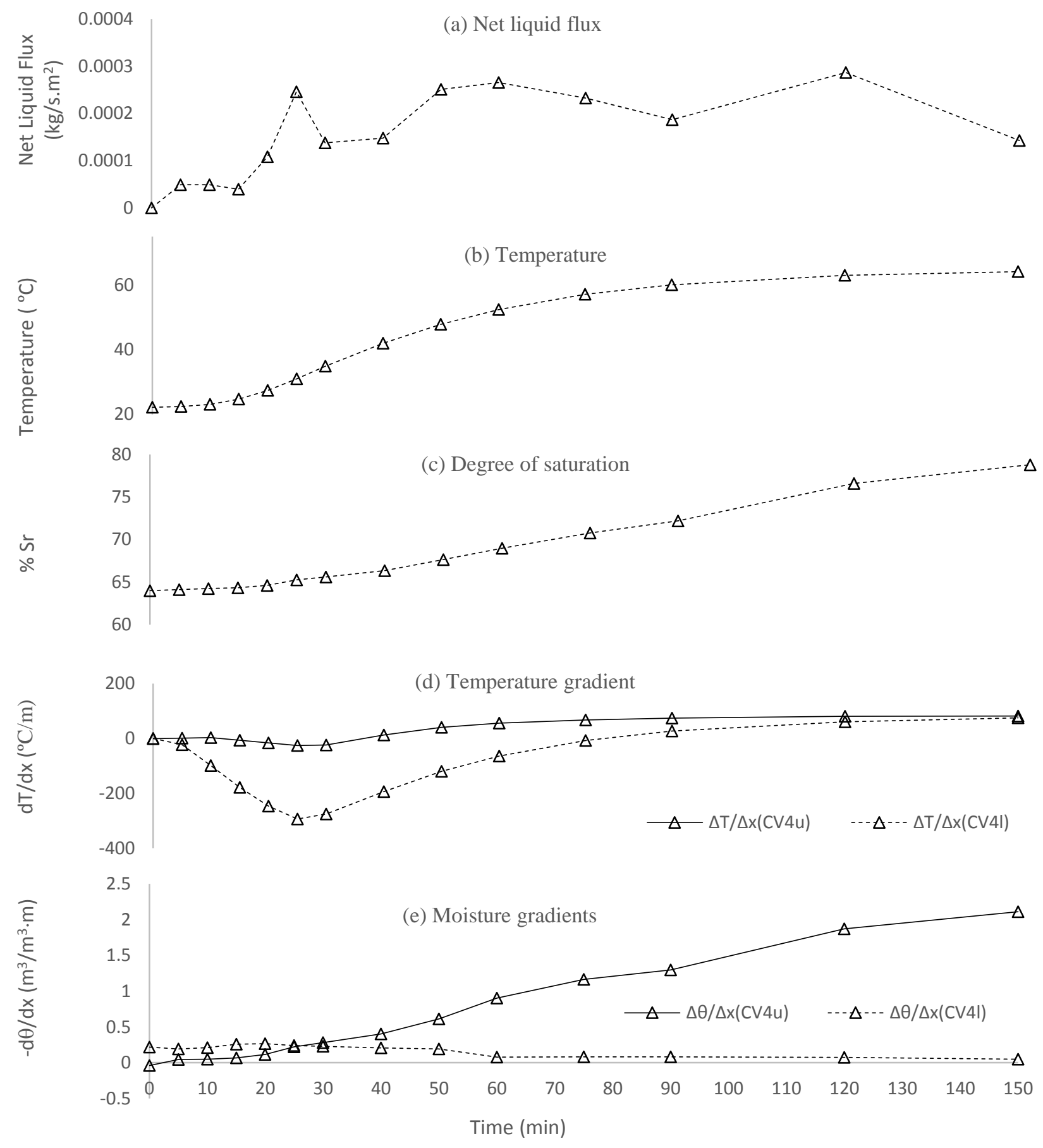

Fig. D.14 The experimental results for control volume 4 of the soil column for the case of $75^{\circ} \mathrm{C}$ hot plate and $60^{\circ} \mathrm{C}$ cold plate 

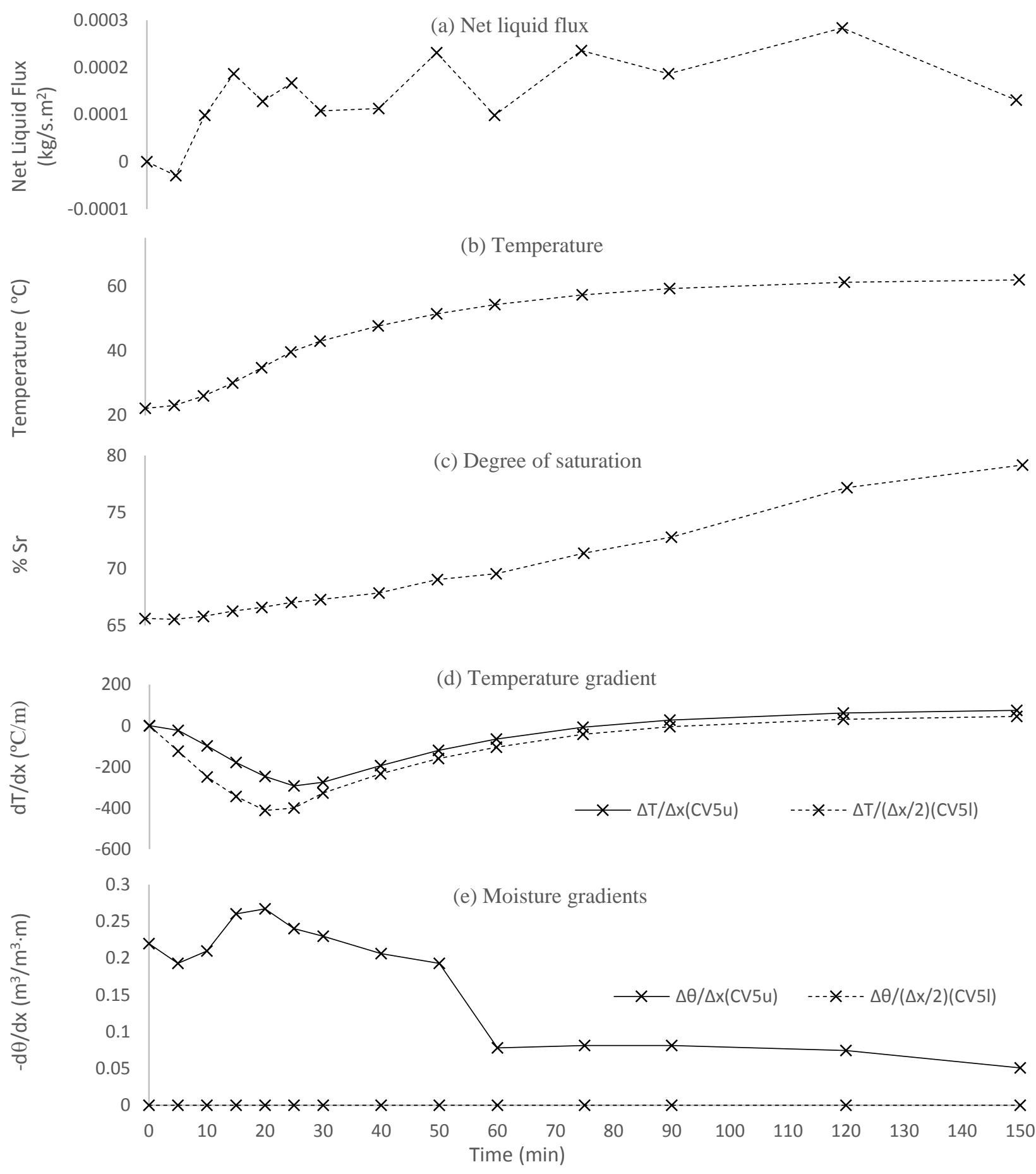

Fig. D.15 The experimental results for control volume 5 of the soil column for the case of $75^{\circ} \mathrm{C}$ hot plate and $60^{\circ} \mathrm{C}$ cold plate 

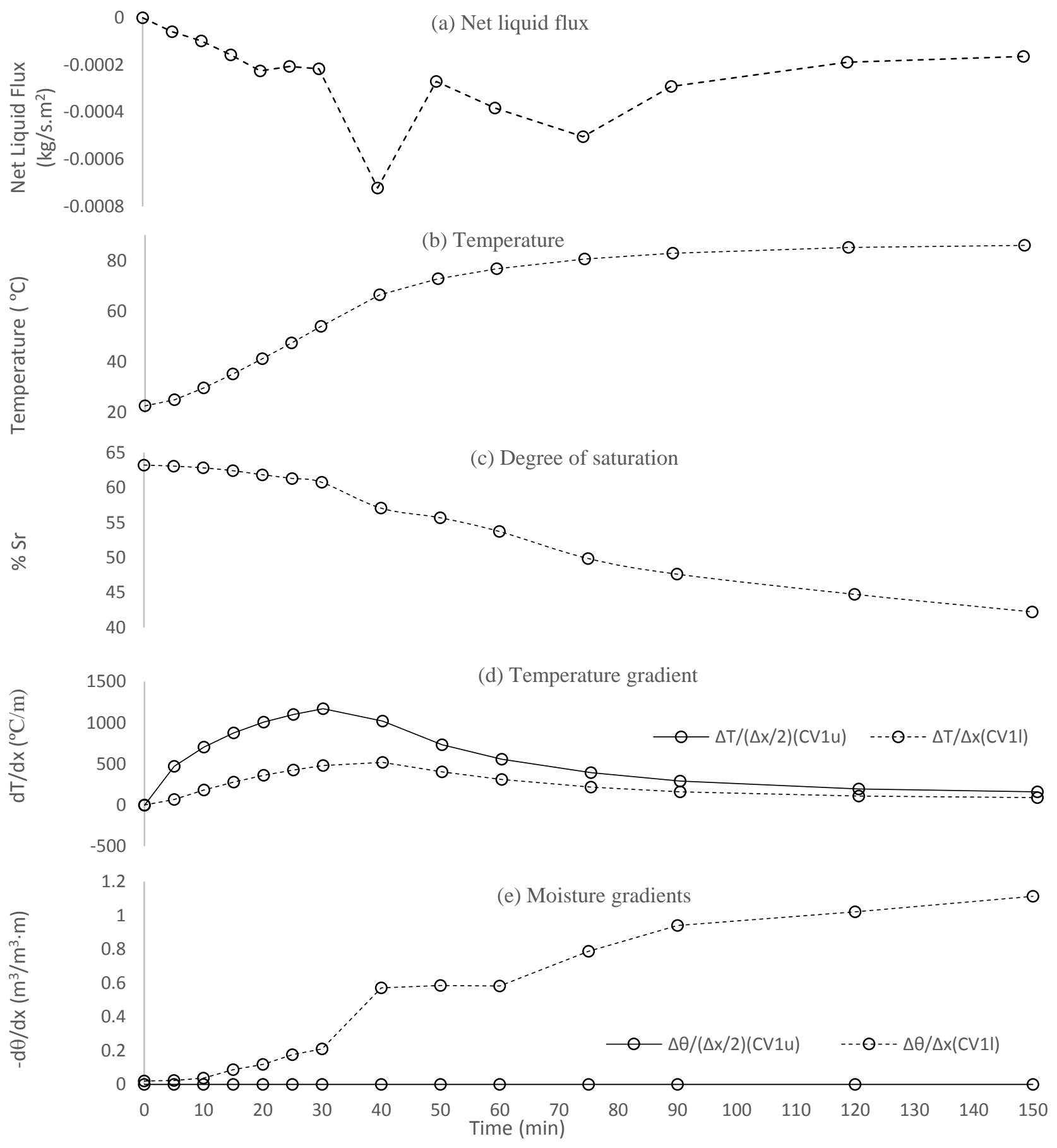

Fig. D.16 The experimental results for control volume 1 of the soil column for the case of $90^{\circ} \mathrm{C}$ hot plate and $75^{\circ} \mathrm{C}$ cold plate 

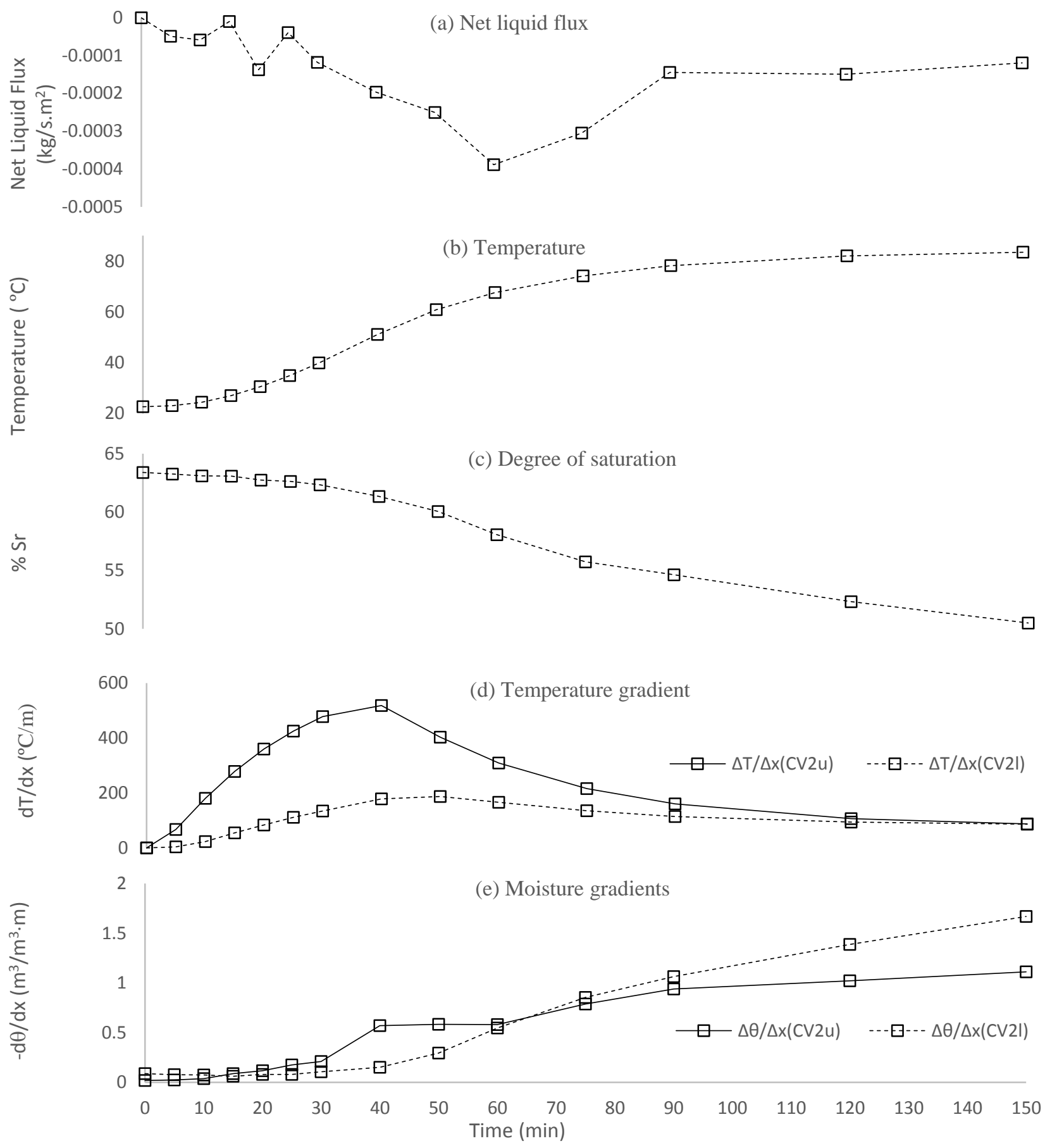

Fig. D.17 The experimental results for control volume 2 of the soil column for the case of $90^{\circ} \mathrm{C}$ hot plate and $75^{\circ} \mathrm{C}$ cold plate 

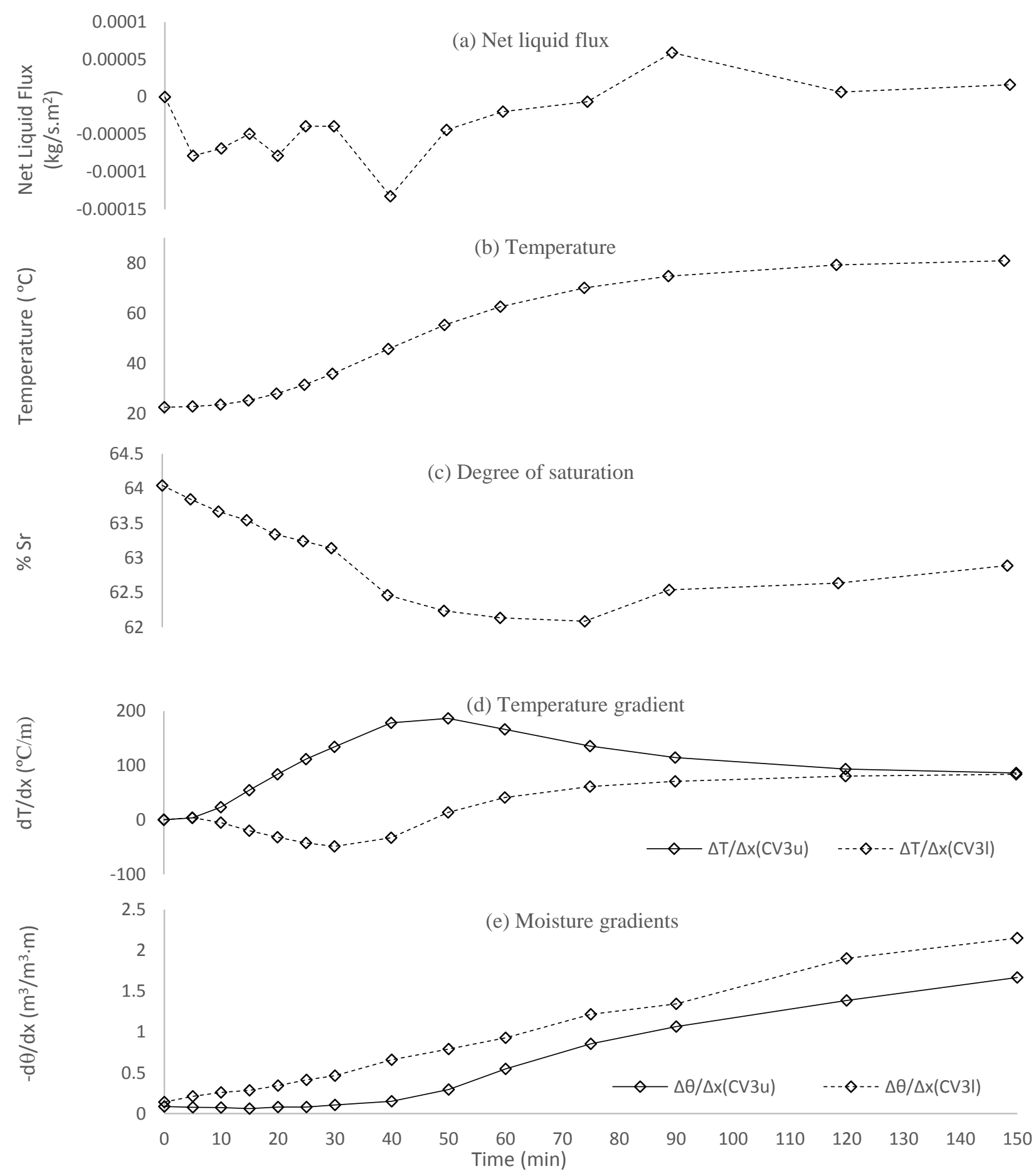

Fig. D.18 The experimental results for control volume 3 of the soil column for the case of $90^{\circ} \mathrm{C}$ hot plate and $75^{\circ} \mathrm{C}$ cold plate 

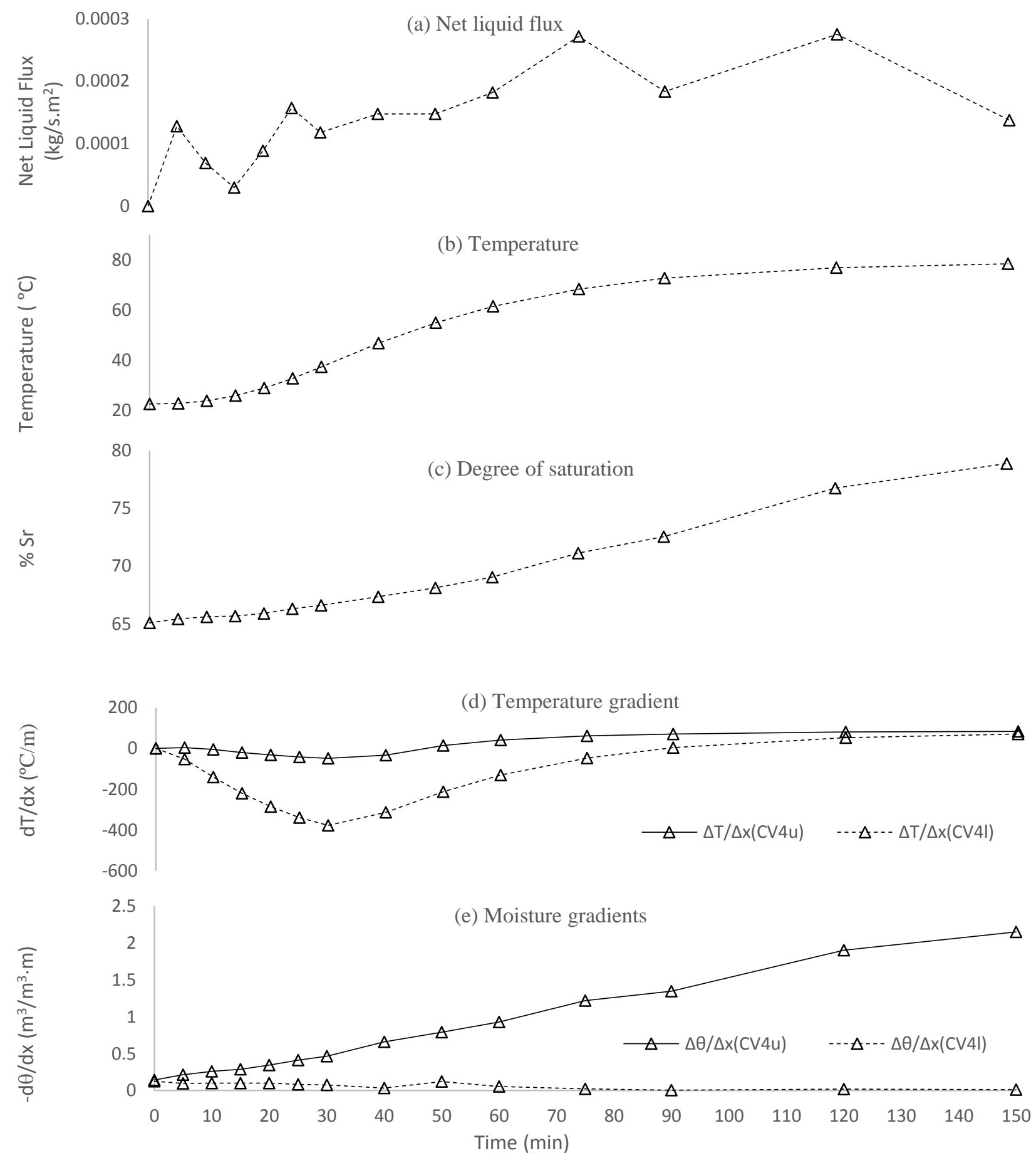

Fig. D.19 The experimental results for control volume 4 of the soil column for the case of $90^{\circ} \mathrm{C}$ hot plate and $75^{\circ} \mathrm{C}$ cold plate 

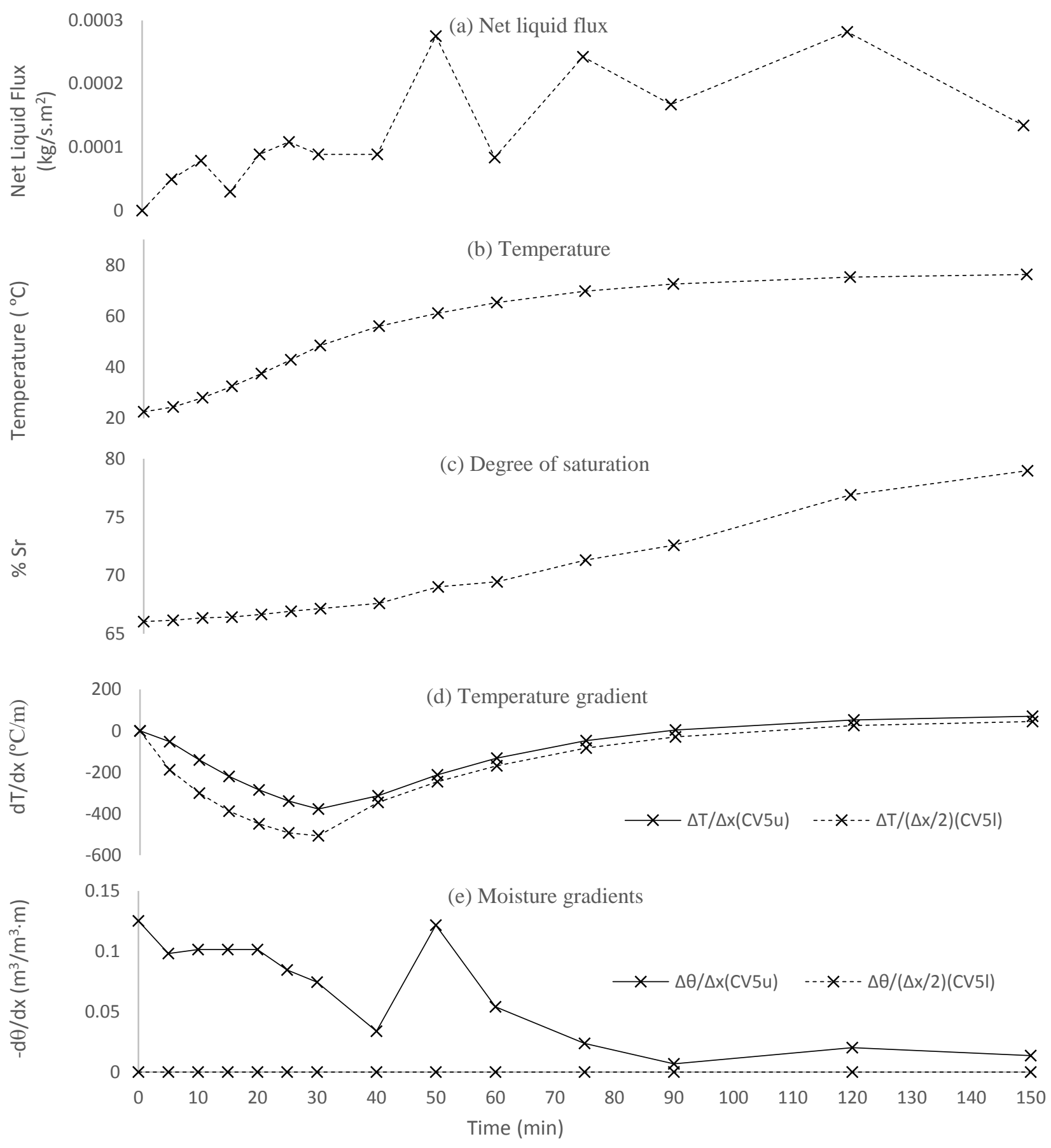

Fig. D.20 The experimental results for control volume 5 of the soil column for the case of $90^{\circ} \mathrm{C}$ hot plate and $75^{\circ} \mathrm{C}$ cold plate 

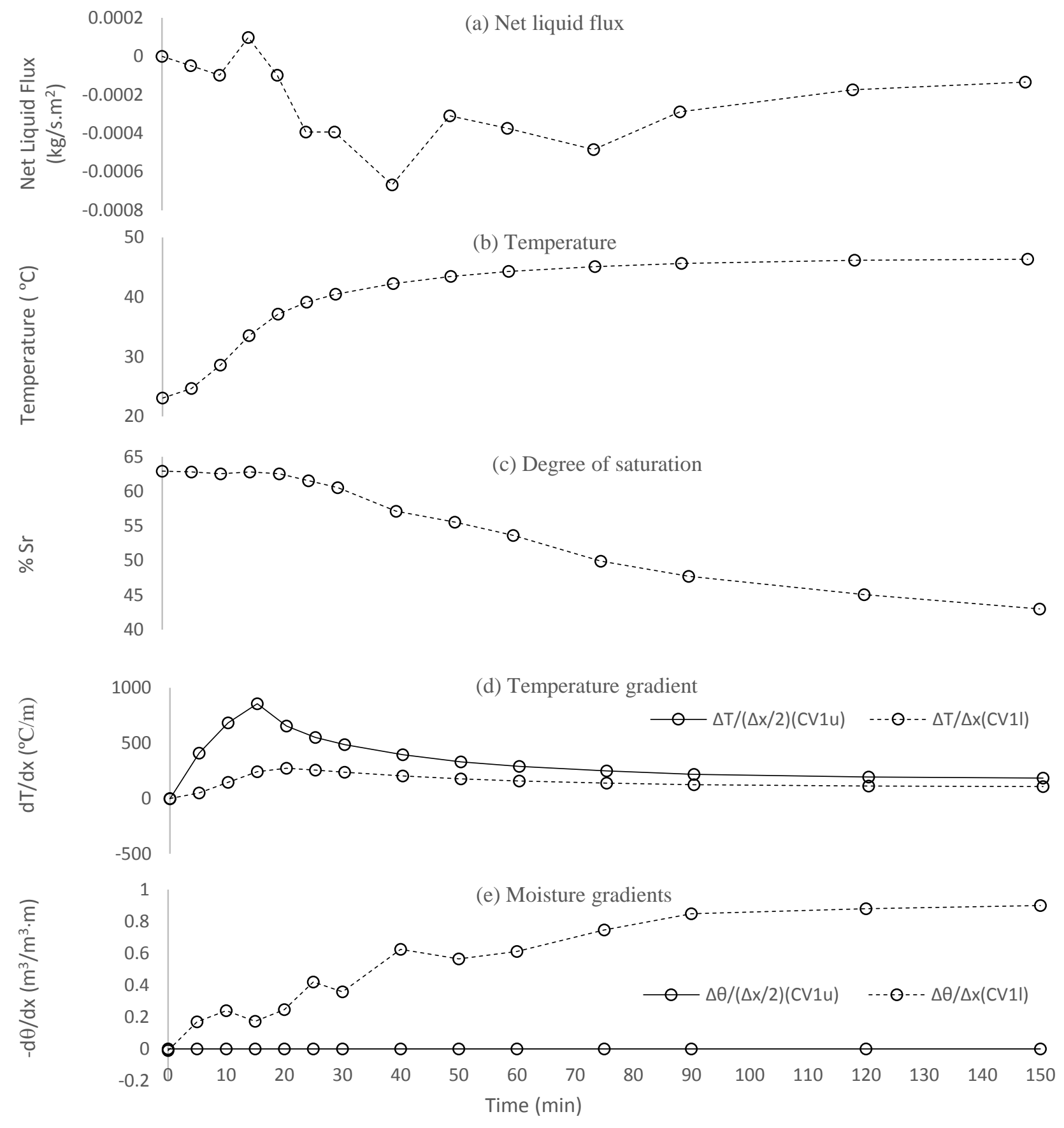

Fig. D.21 The experimental results for control volume 1 of the soil column for the case of $50^{\circ} \mathrm{C}$ hot plate and $30^{\circ} \mathrm{C}$ cold plate 

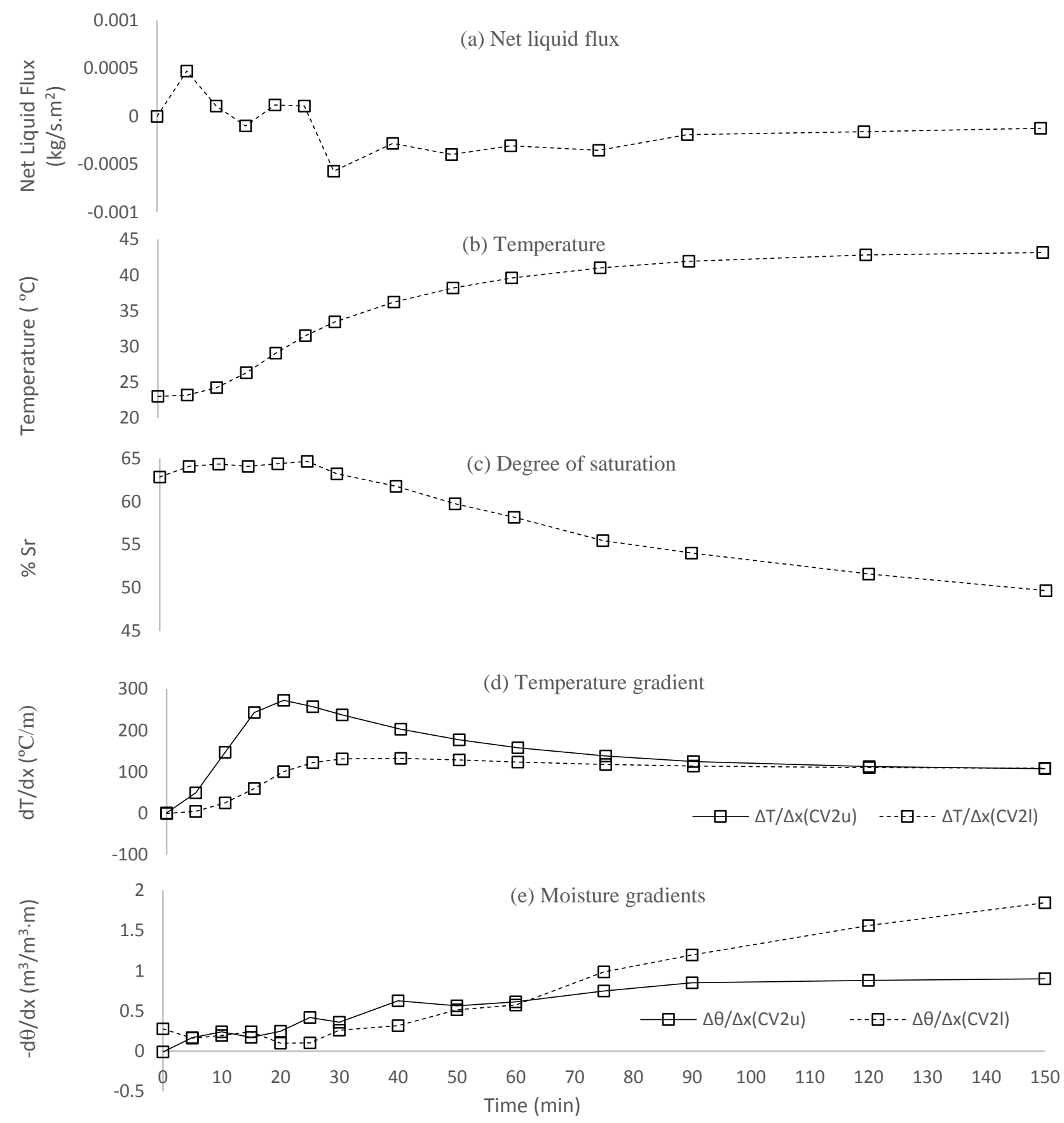

Fig. D.22 The experimental results for control volume 2 of the soil column for the case of $50^{\circ} \mathrm{C}$ hot plate and $30^{\circ} \mathrm{C}$ cold plate 

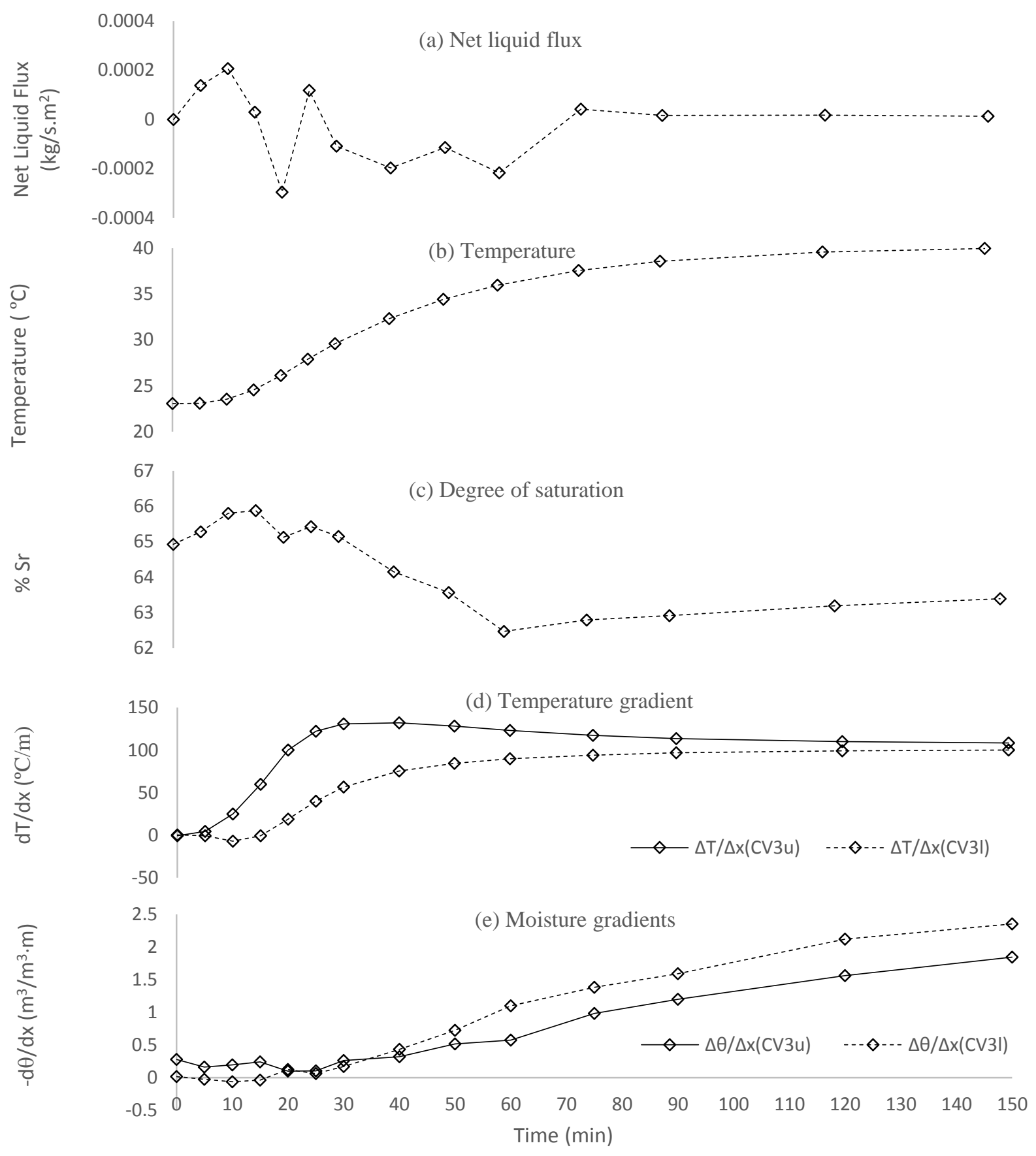

Fig. D.23 The experimental results for control volume 3 of the soil column for the case of $50^{\circ} \mathrm{C}$ hot plate and $30^{\circ} \mathrm{C}$ cold plate 

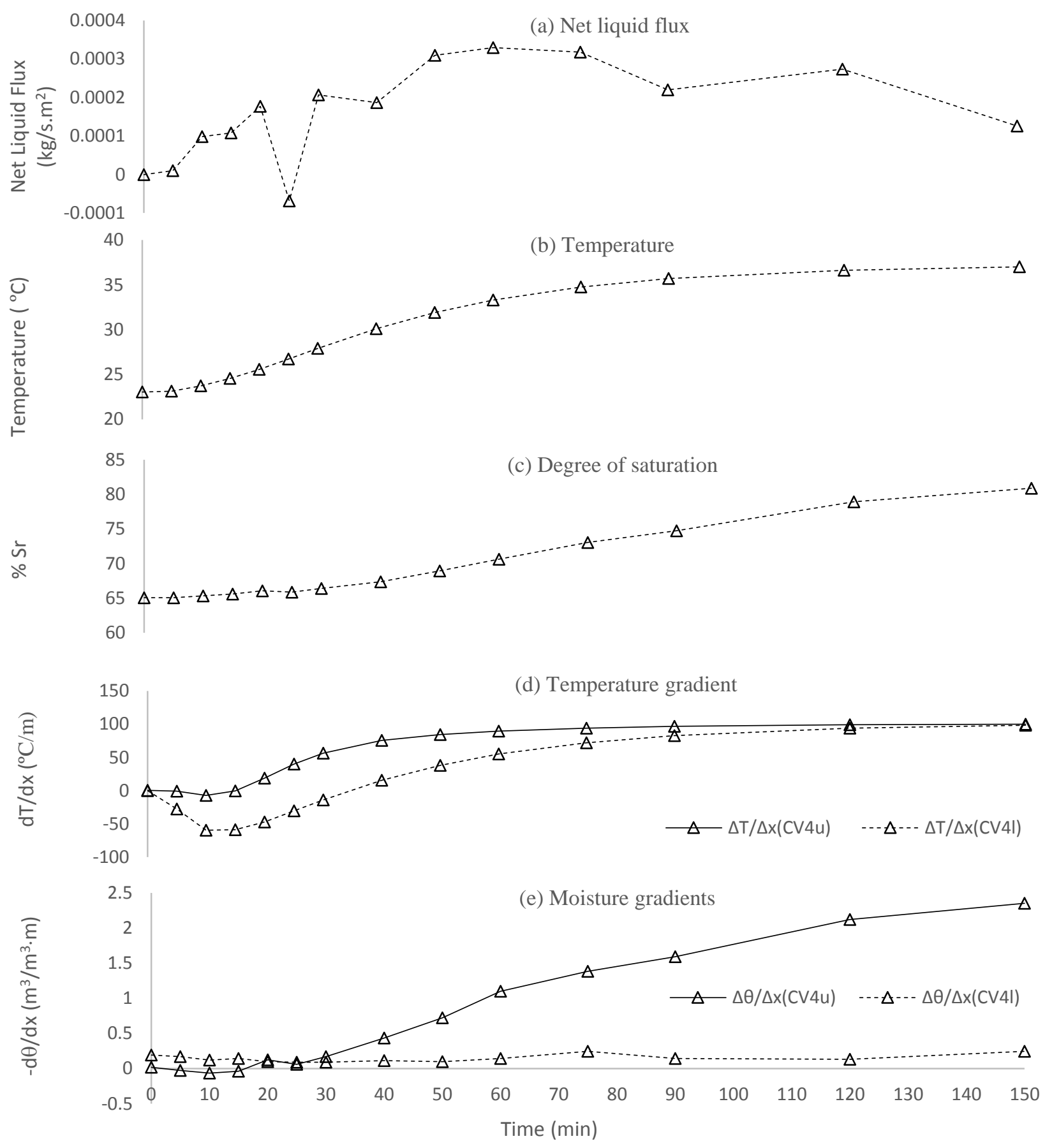

Fig. D.24 The experimental results for control volume 4 of the soil column for the case of $50^{\circ} \mathrm{C}$ hot plate and $30^{\circ} \mathrm{C}$ cold plate 

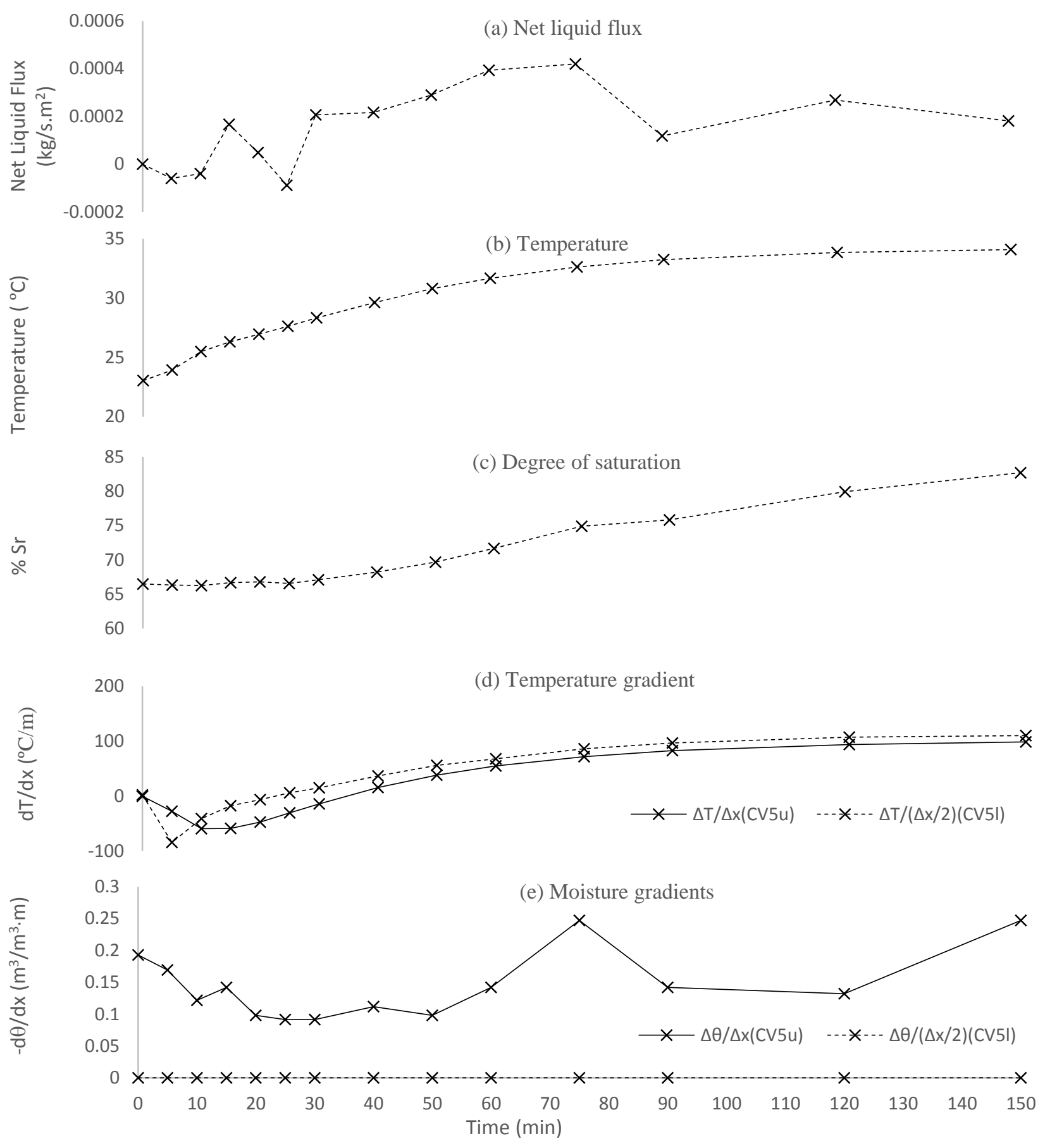

Fig. D.25 The experimental results for control volume 5 of the soil column for the case of $50^{\circ} \mathrm{C}$ hot plate and $30^{\circ} \mathrm{C}$ cold plate 

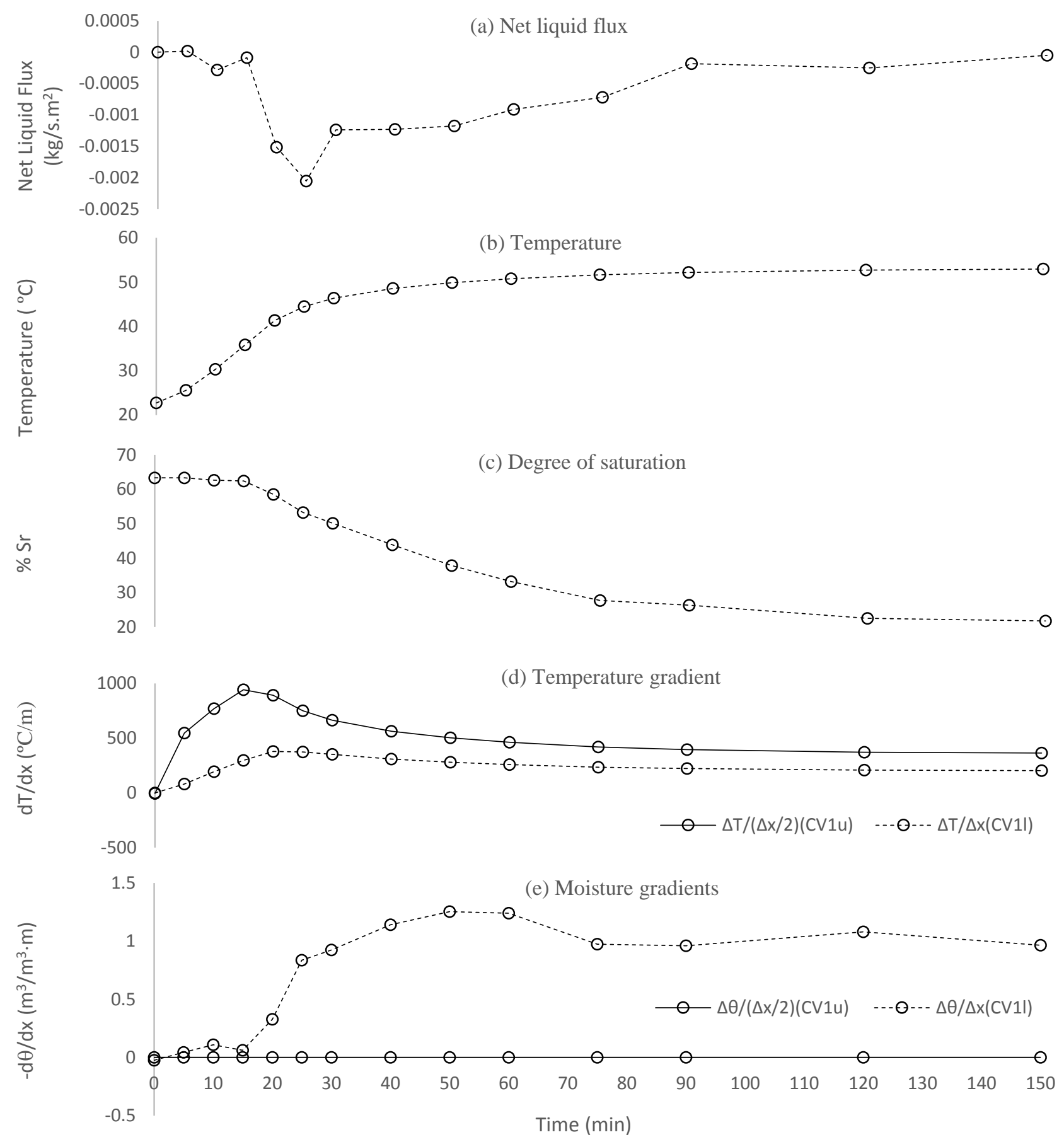

Fig. D.26 The experimental results for control volume 1 of the soil column for the case of $60^{\circ} \mathrm{C}$ hot plate and $20^{\circ} \mathrm{C}$ cold plate 

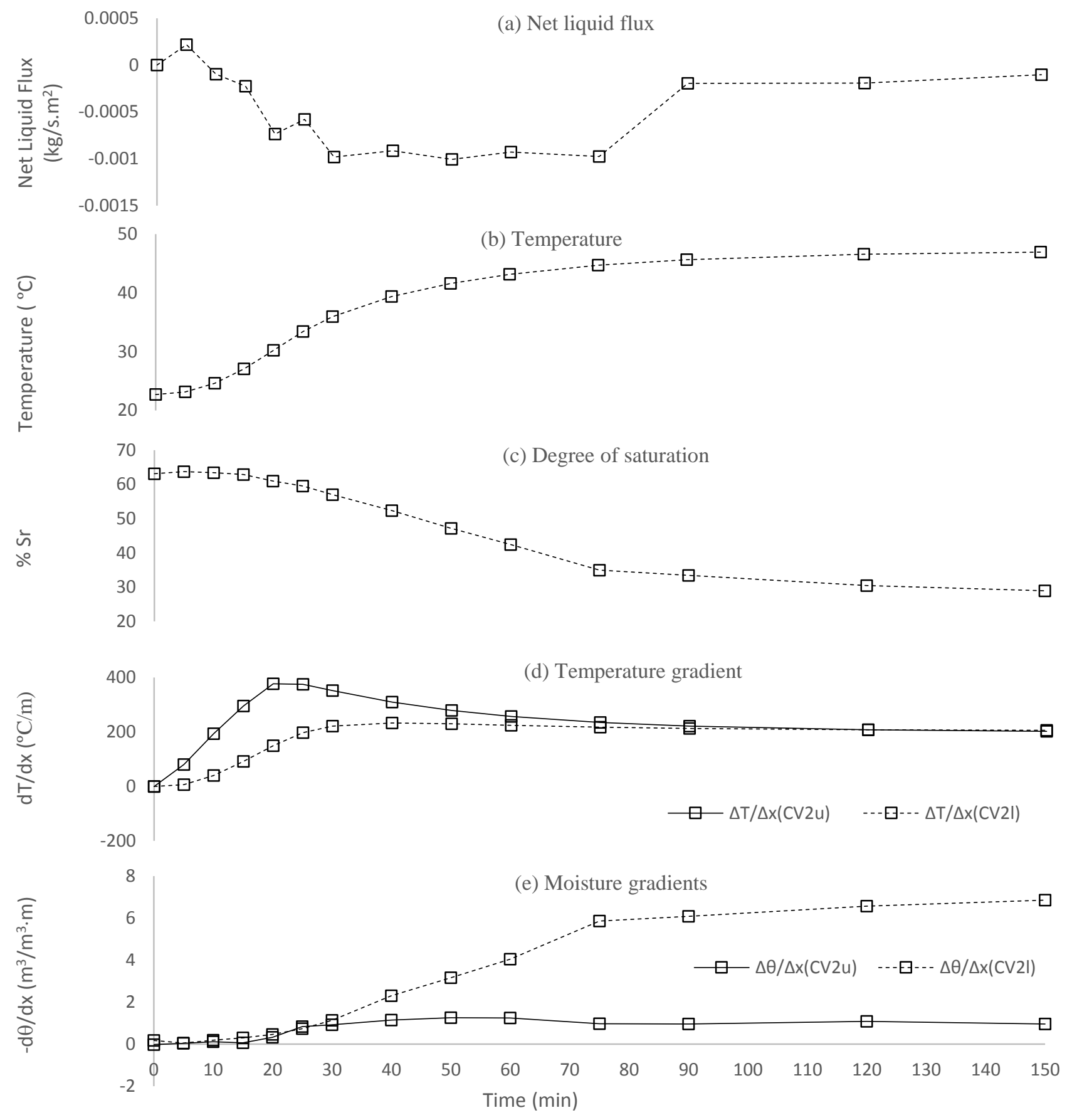

Fig. D.27 The experimental results for control volume 2 of the soil column for the case of $60^{\circ} \mathrm{C}$ hot plate and $20^{\circ} \mathrm{C}$ cold plate 

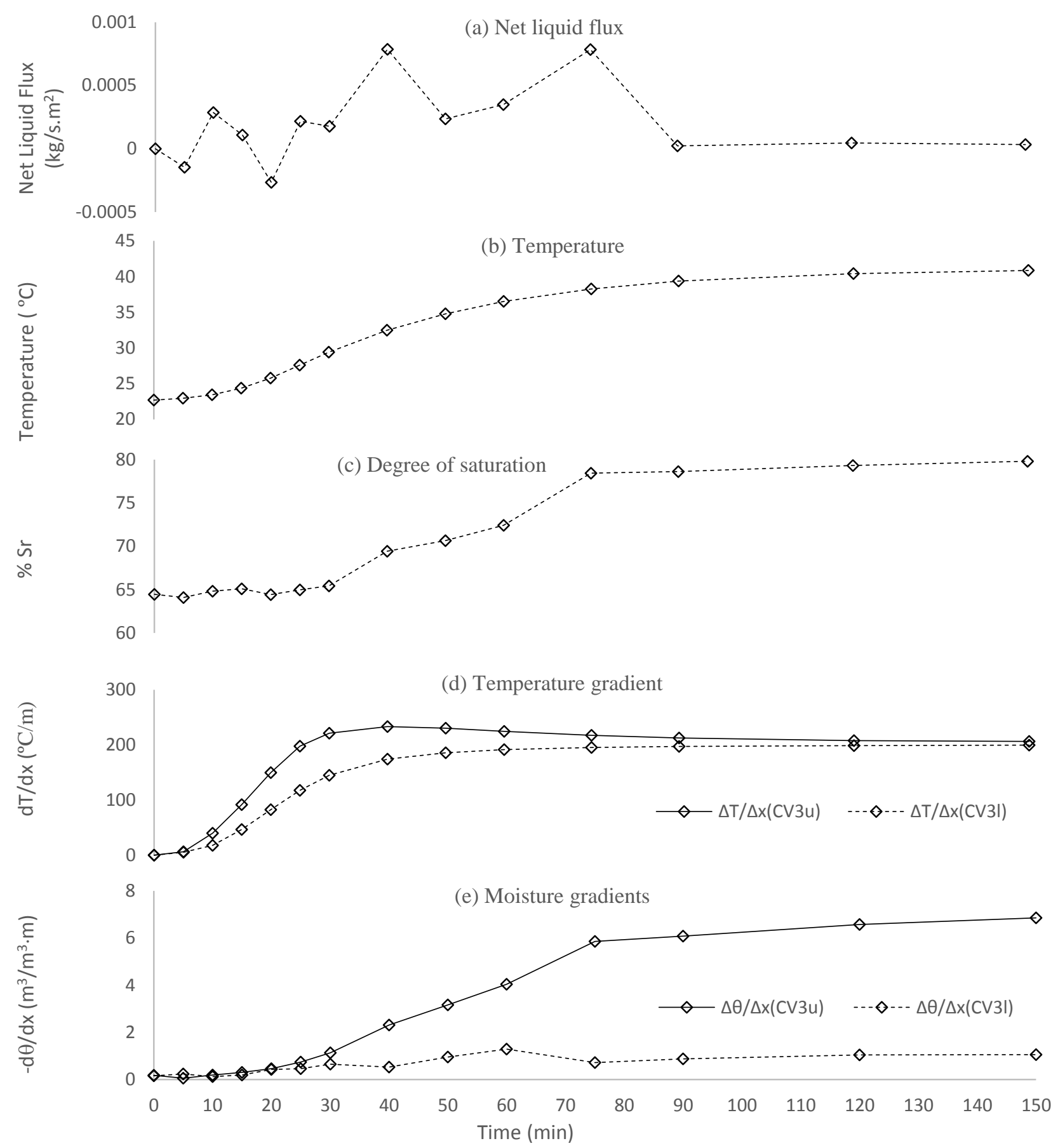

Fig. D.28 The experimental results for control volume 3 of the soil column for the case of $60^{\circ} \mathrm{C}$ hot plate and $20^{\circ} \mathrm{C}$ cold plate 


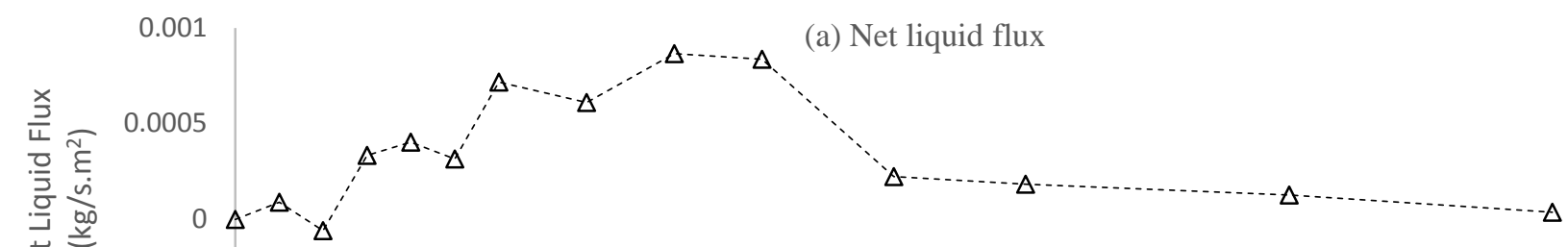

$z$
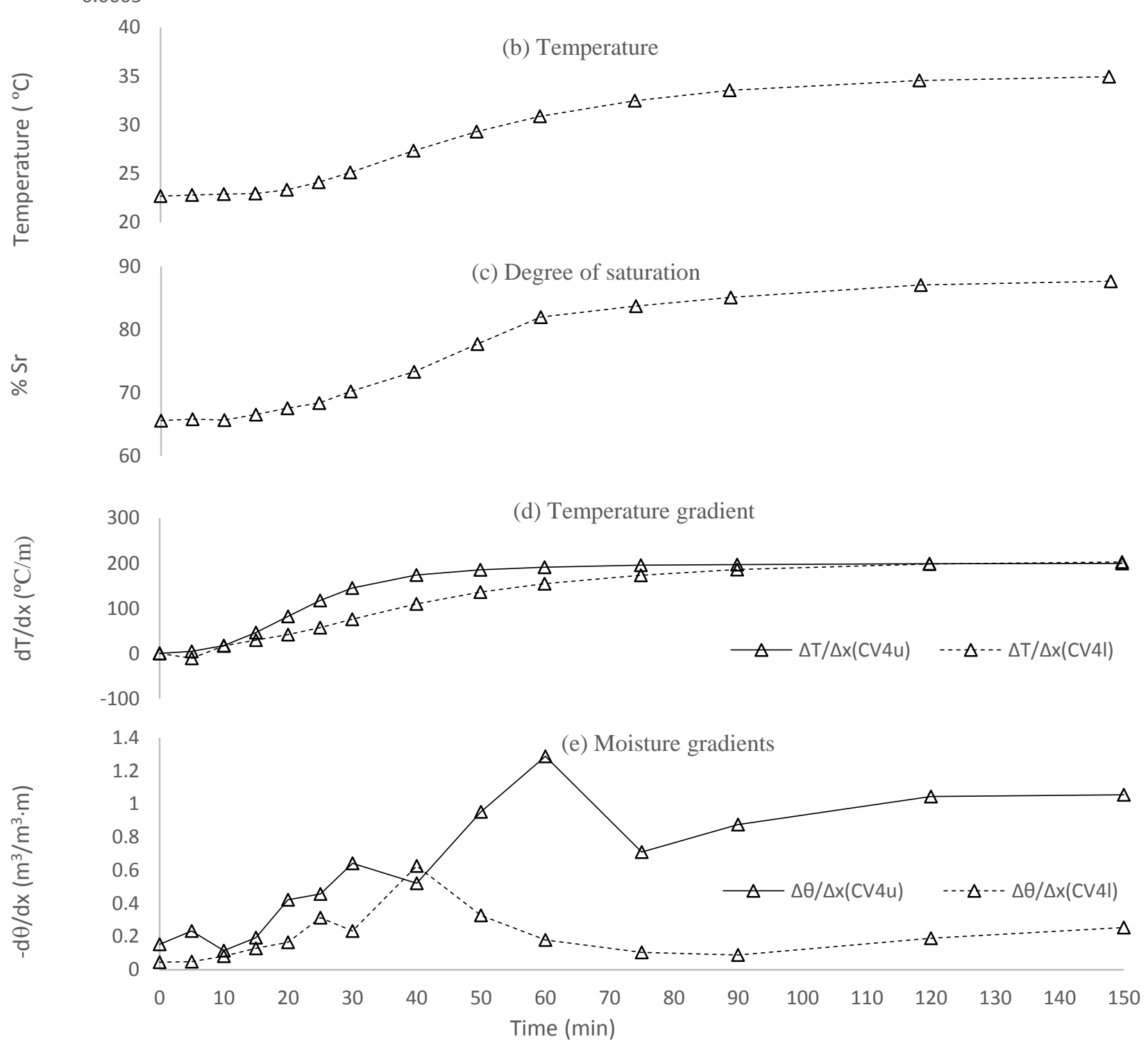

Fig. D.29 The experimental results for control volume 4 of the soil column for the case of $60^{\circ} \mathrm{C}$ hot plate and $20^{\circ} \mathrm{C}$ cold plate 

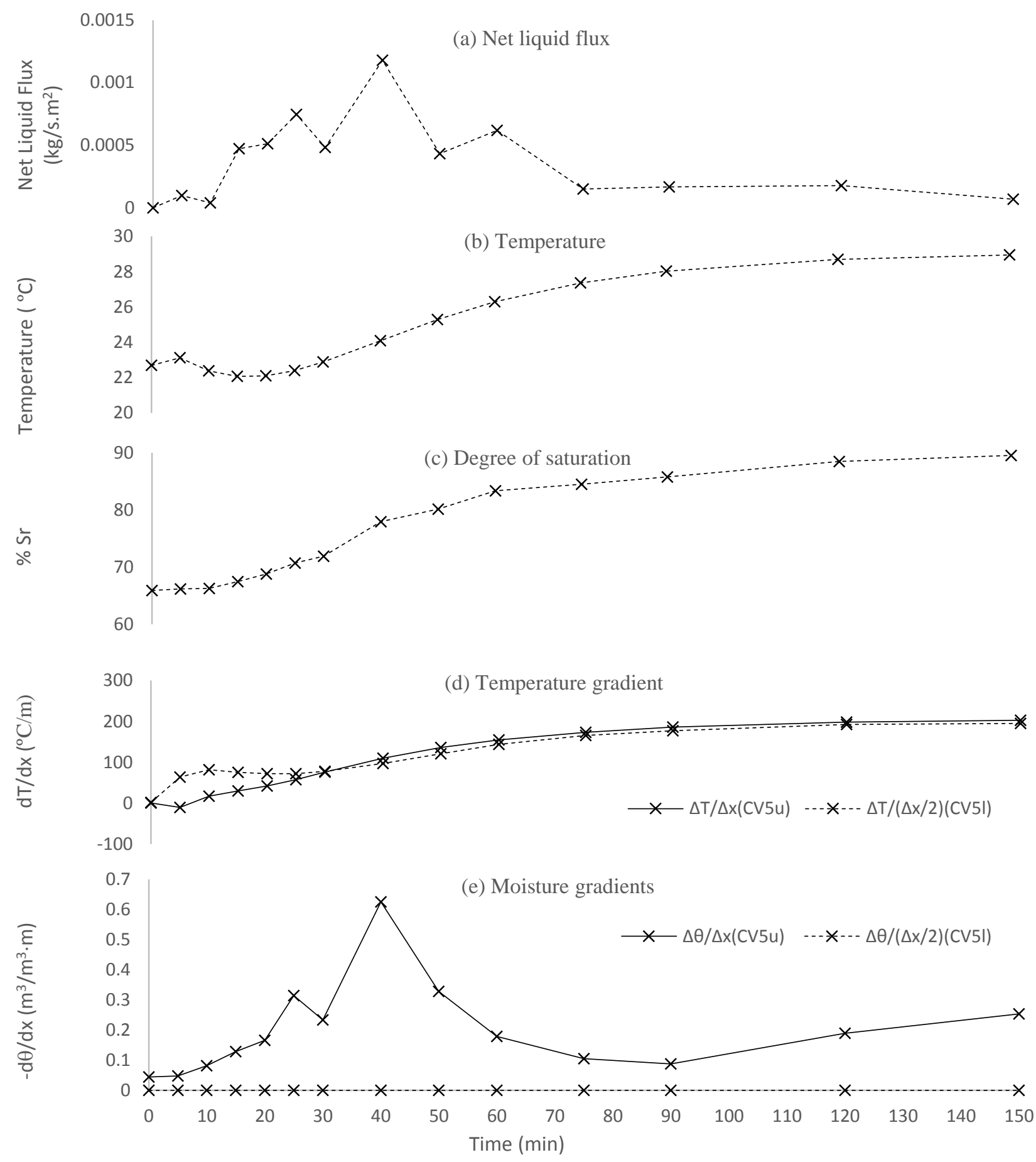

Fig. D.30 The experimental results for control volume 5 of the soil column for the case of $60^{\circ} \mathrm{C}$ hot plate and $20^{\circ} \mathrm{C}$ cold plate 

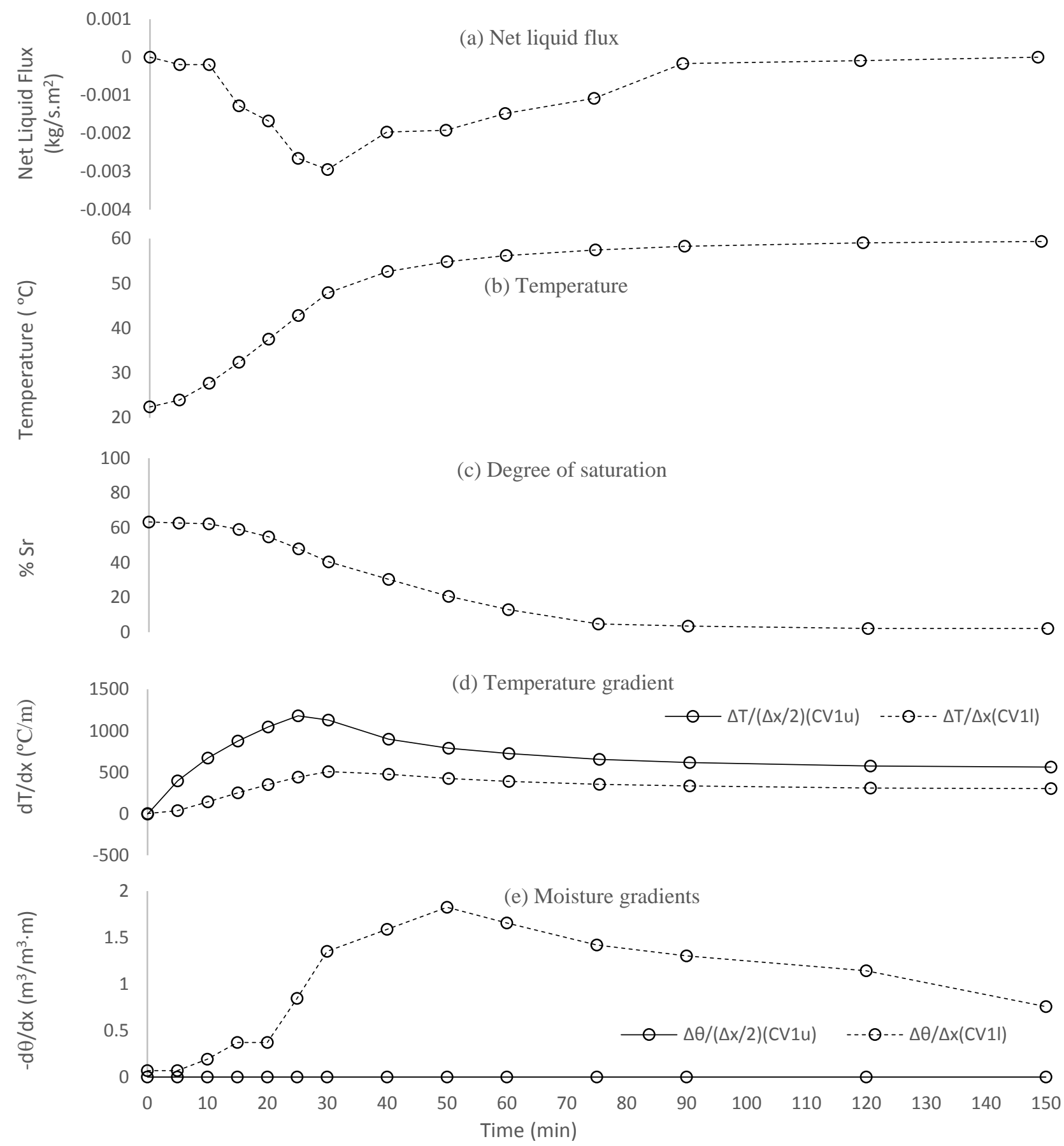

Fig. D.31 The experimental results for control volume 1 of the soil column for the case of $70^{\circ} \mathrm{C}$ hot plate and $10^{\circ} \mathrm{C}$ cold plate 

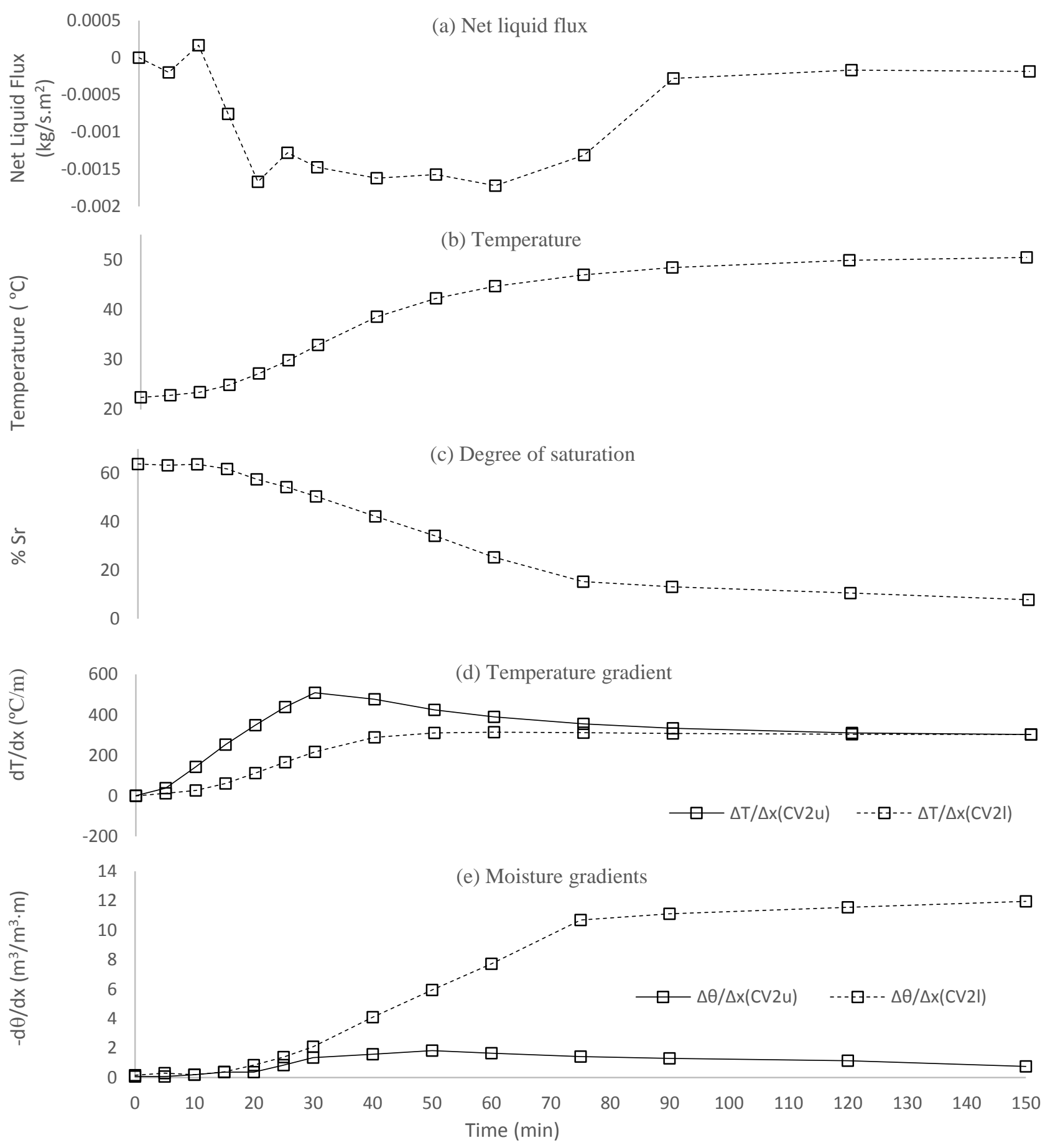

Fig. D.32 The experimental results for control volume 2 of the soil column for the case of $70^{\circ} \mathrm{C}$ hot plate and $10^{\circ} \mathrm{C}$ cold plate 

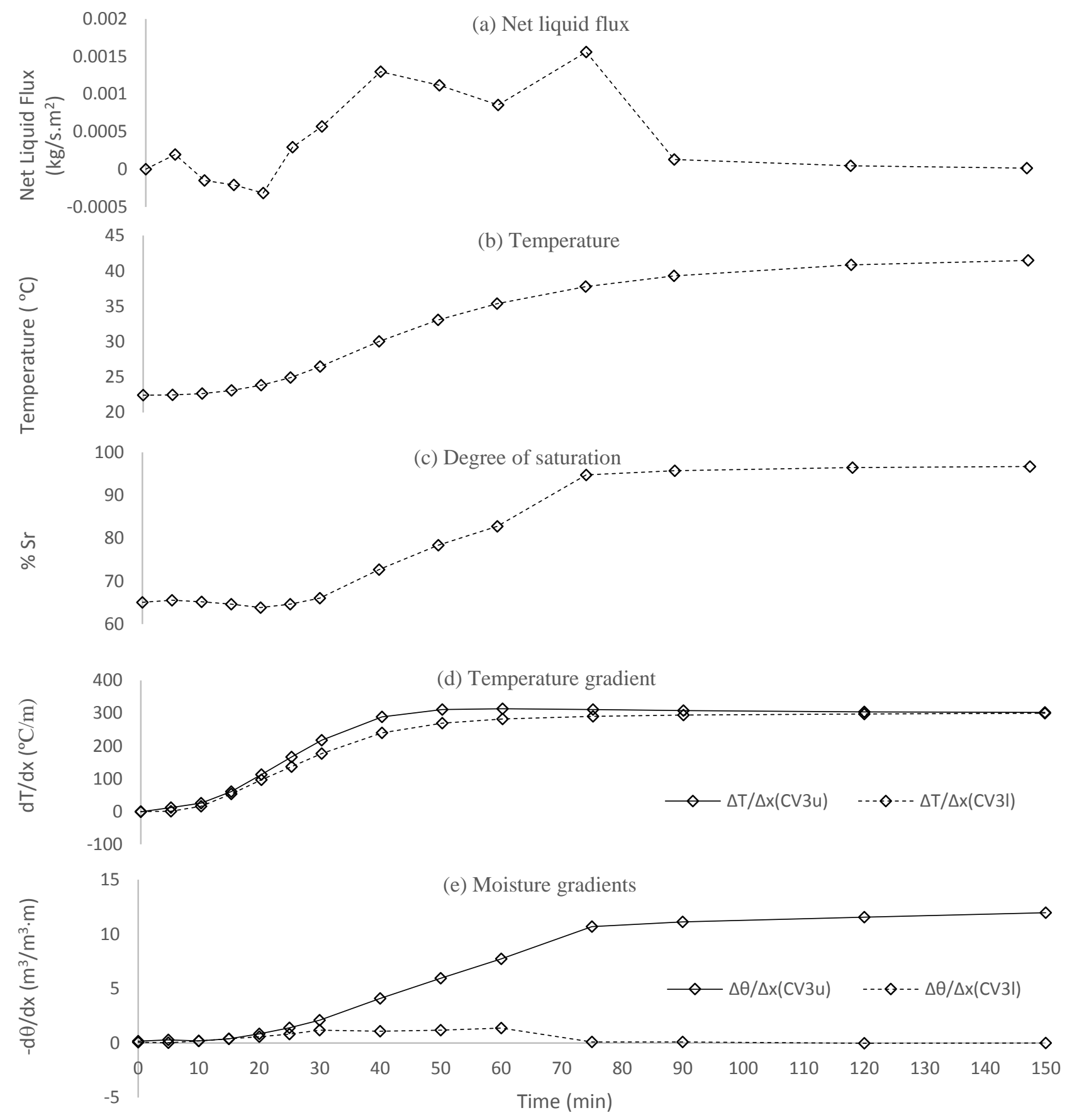

Fig. D.33 The experimental results for control volume 3 of the soil column for the case of $70^{\circ} \mathrm{C}$ hot plate and $10^{\circ} \mathrm{C}$ cold plate 

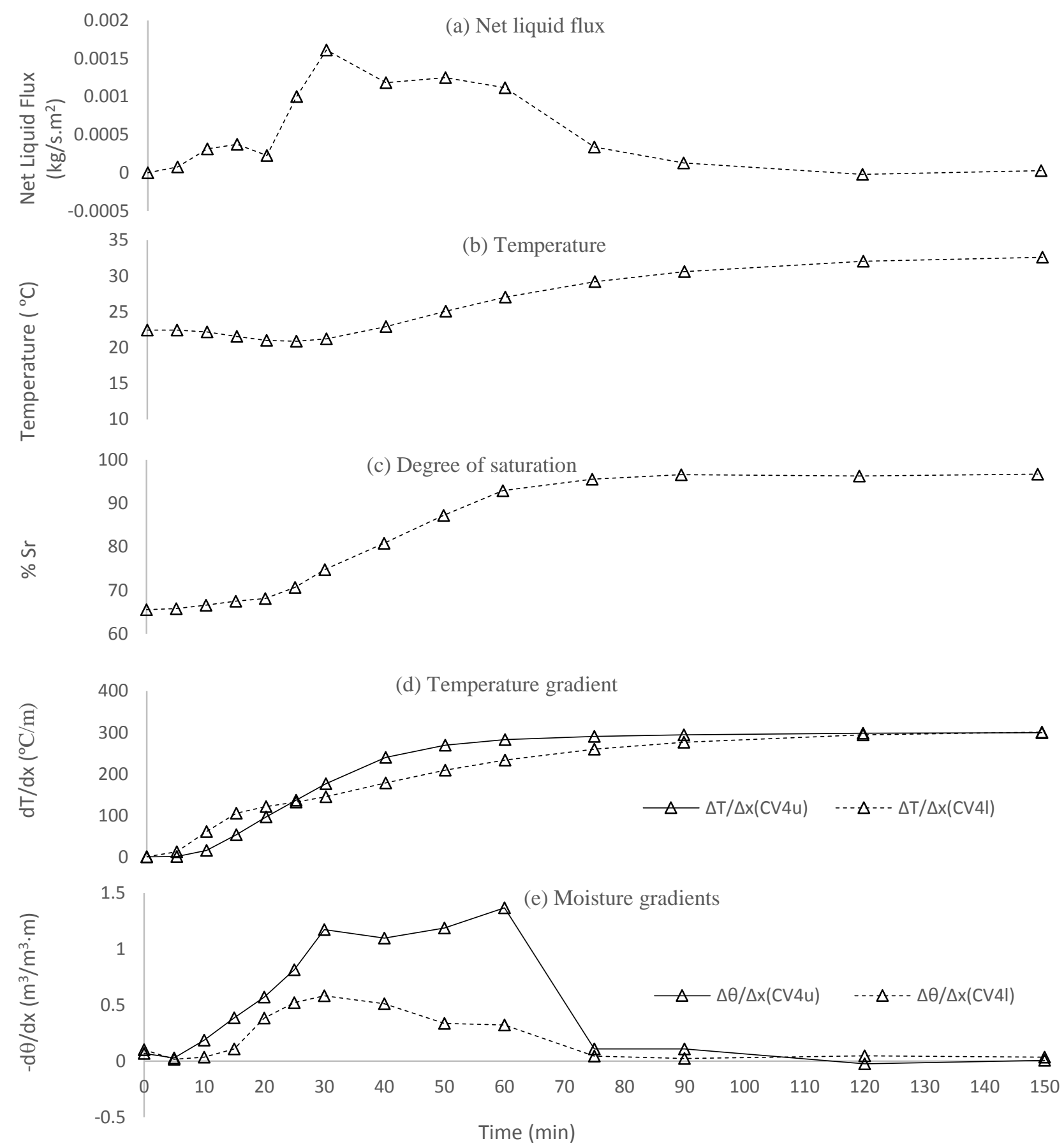

Fig. D.34 The experimental results for control volume 4 of the soil column for the case of $70^{\circ} \mathrm{C}$ hot plate and $10^{\circ} \mathrm{C}$ cold plate 

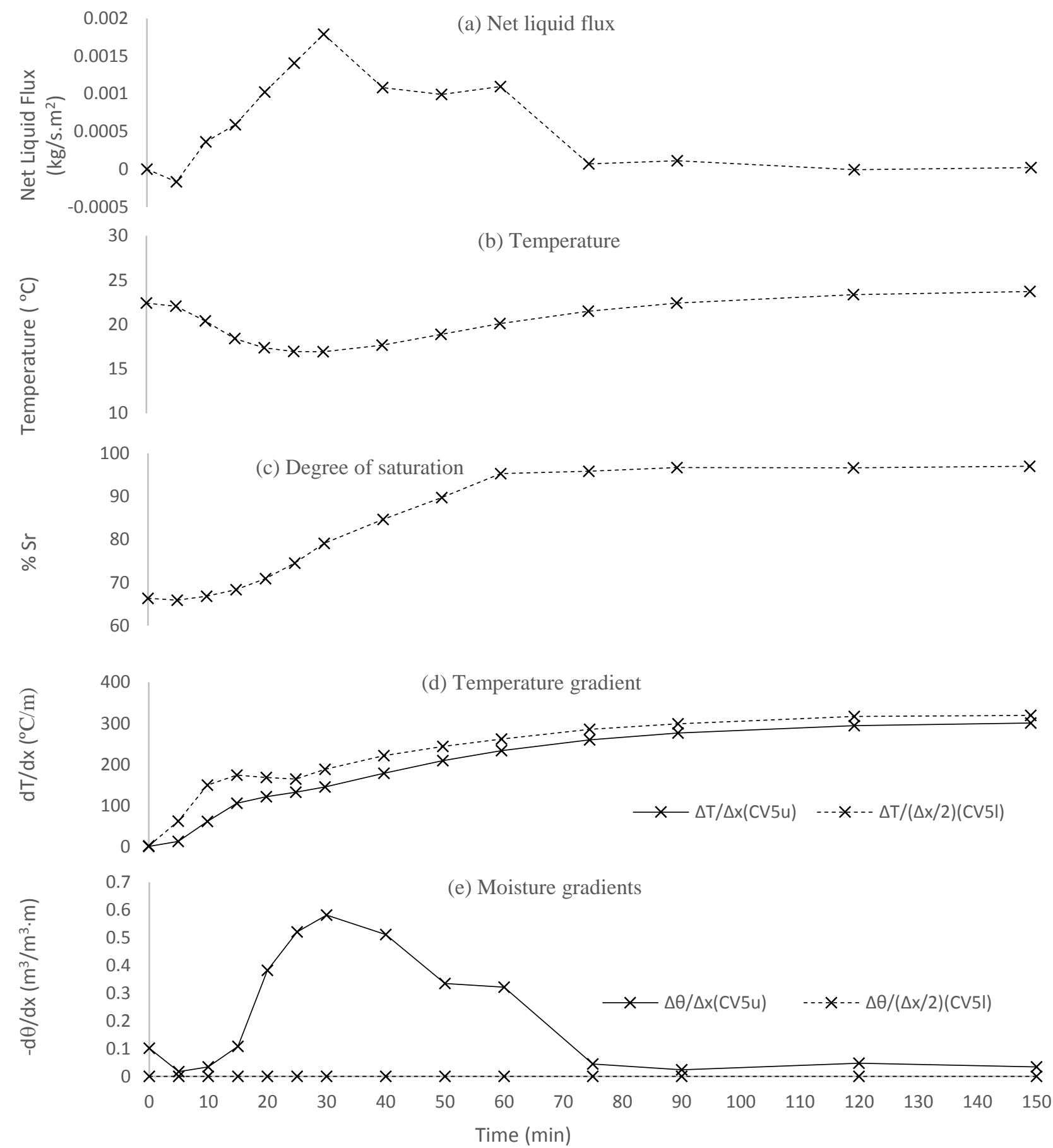

Fig. D.35 The experimental results for control volume 5 of the soil column for the case of $70^{\circ} \mathrm{C}$ hot plate and $10^{\circ} \mathrm{C}$ cold plate 

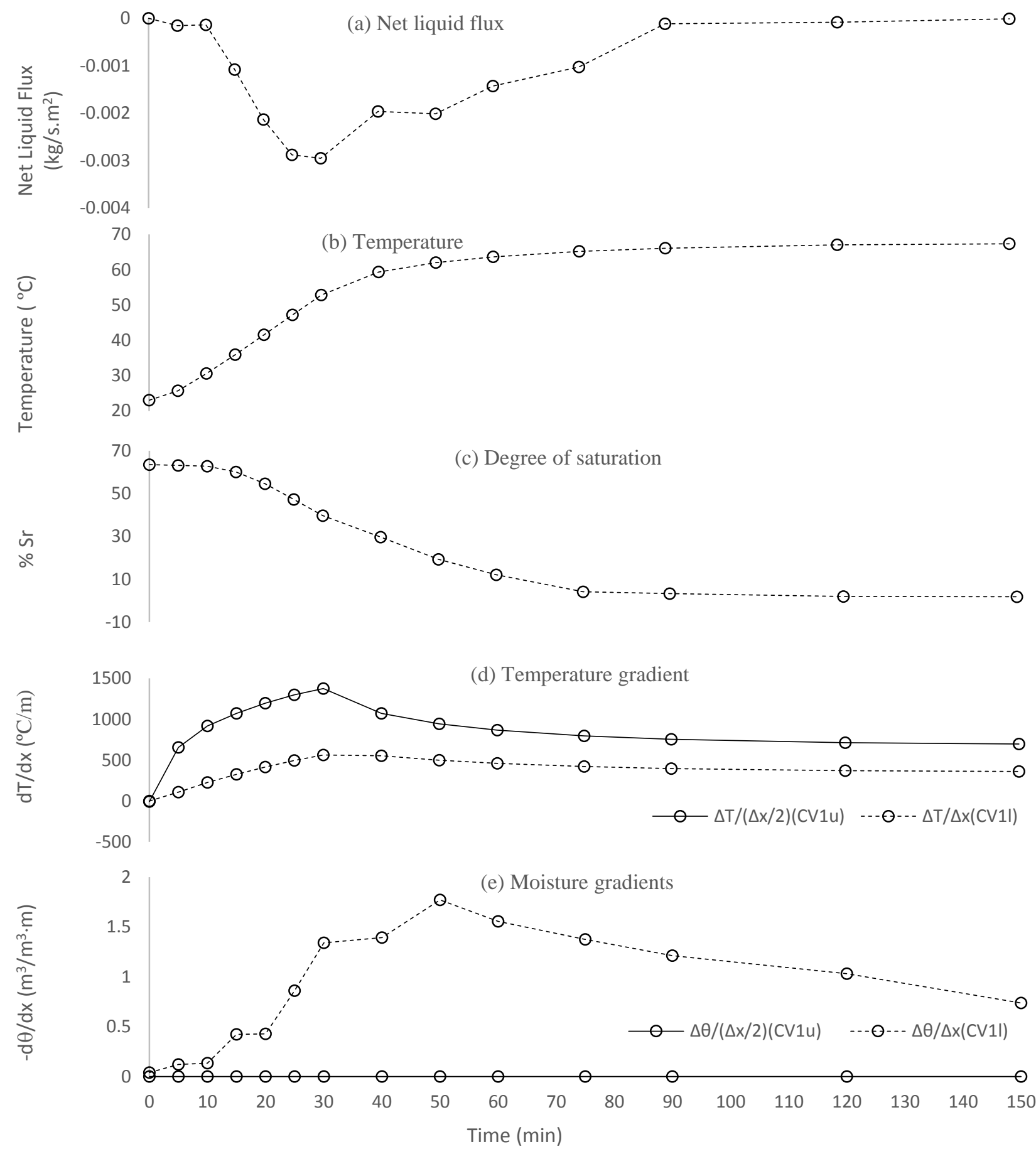

Fig. D.36 The experimental results for control volume 1 of the soil column for the case of $80^{\circ} \mathrm{C}$ hot plate and $10^{\circ} \mathrm{C}$ cold plate 


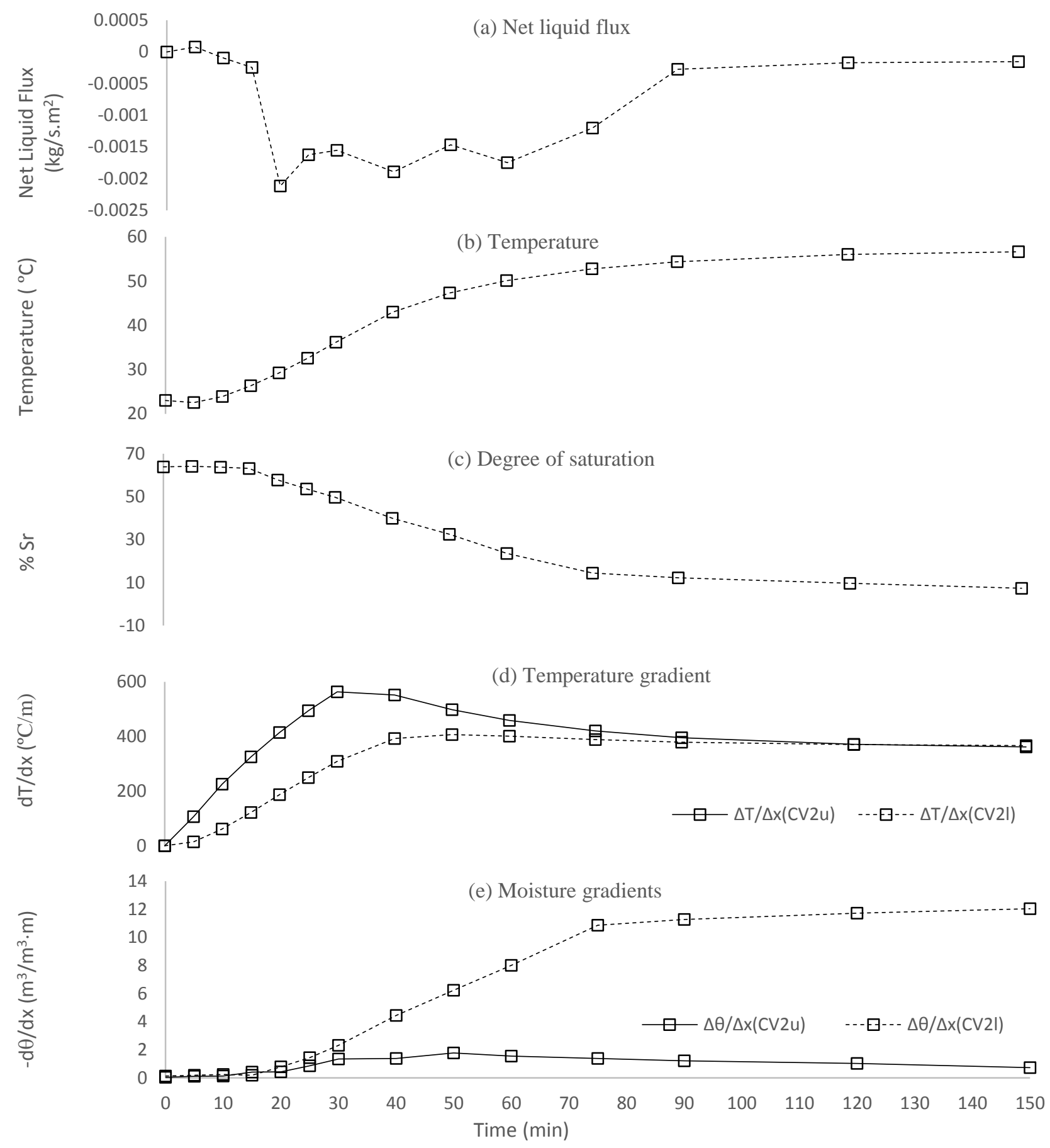

Fig. D.37 The experimental results for control volume 2 of the soil column for the case of $80^{\circ} \mathrm{C}$ hot plate and $10^{\circ} \mathrm{C}$ cold plate 

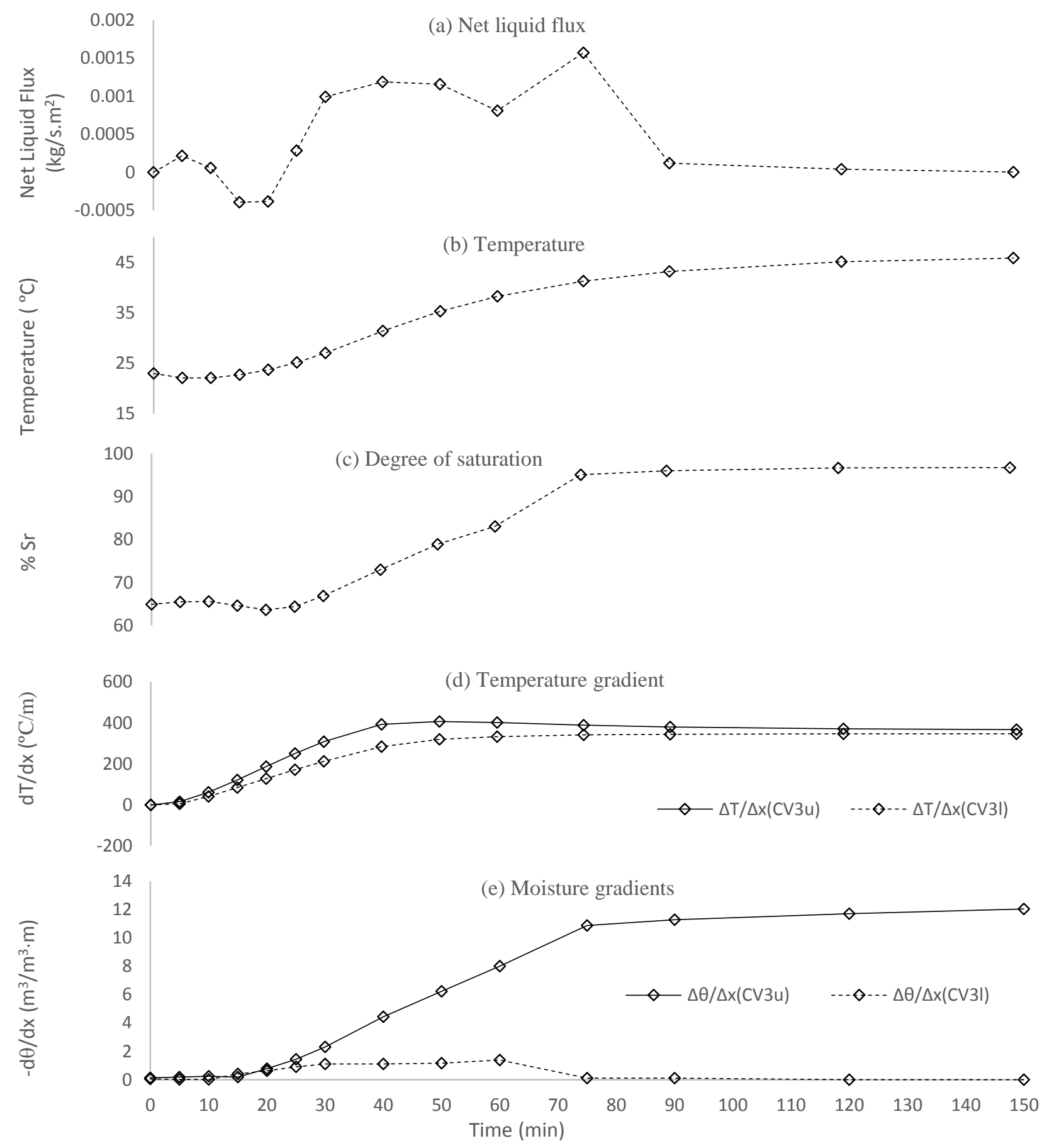

Fig. D.38 The experimental results for control volume 3 of the soil column for the case of $80^{\circ} \mathrm{C}$ hot plate and $10^{\circ} \mathrm{C}$ cold plate 

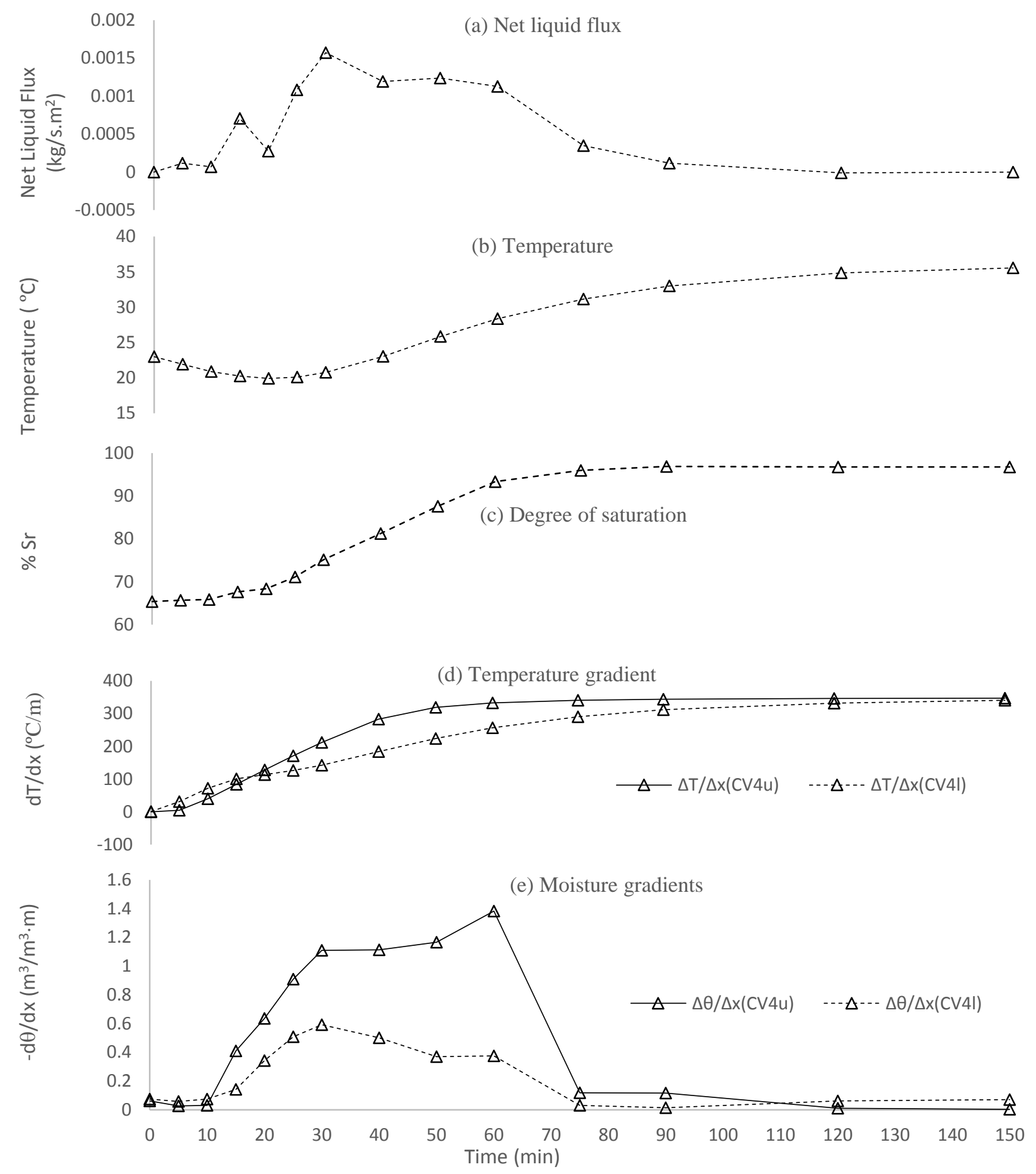

Fig. D.39 The experimental results for control volume 4 of the soil column for the case of $80^{\circ} \mathrm{C}$ hot plate and $10^{\circ} \mathrm{C}$ cold plate 

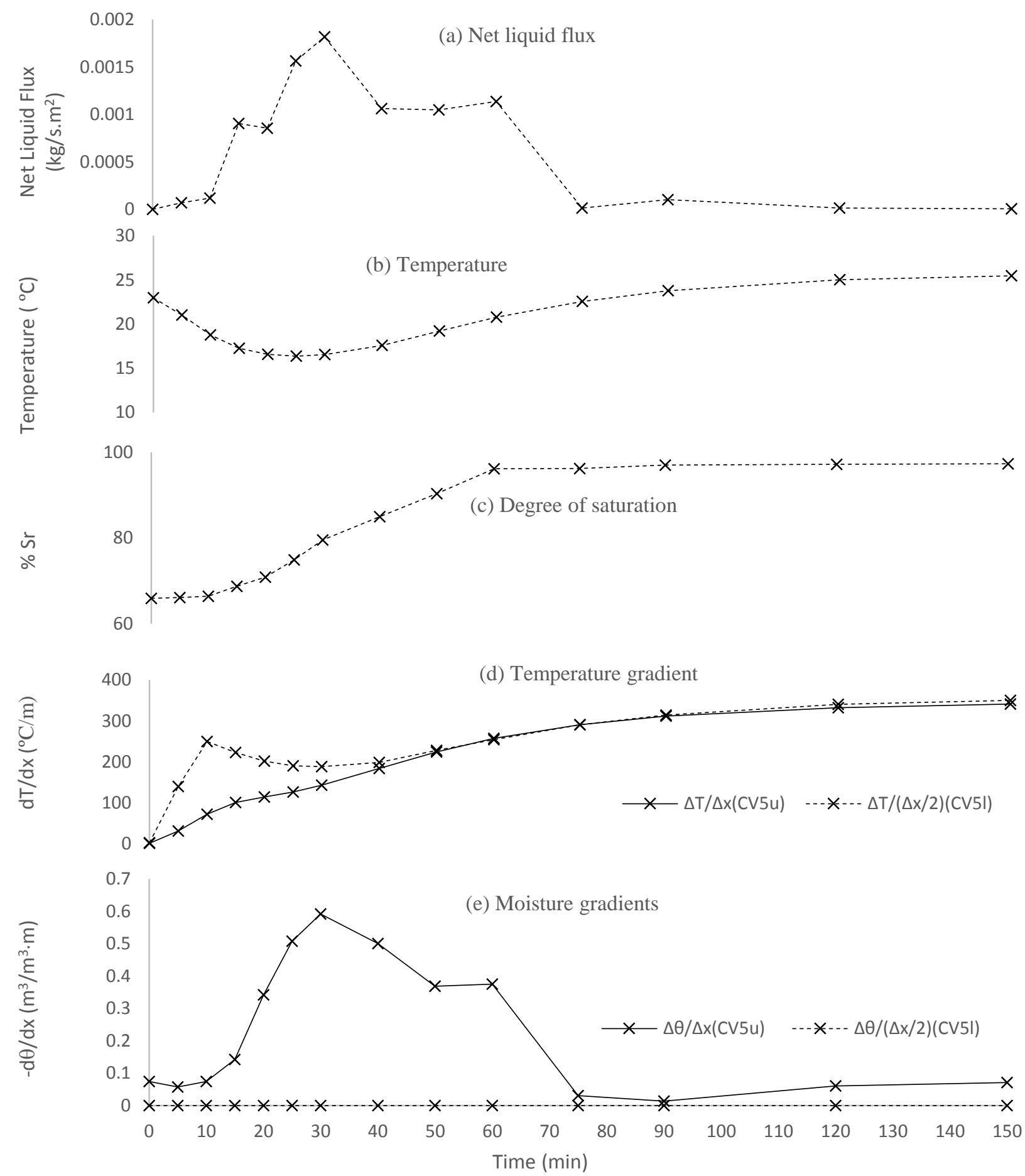

Fig. D.40 The experimental results for control volume 5 of the soil column for the case of $80^{\circ} \mathrm{C}$ hot plate and $10^{\circ} \mathrm{C}$ cold plate 


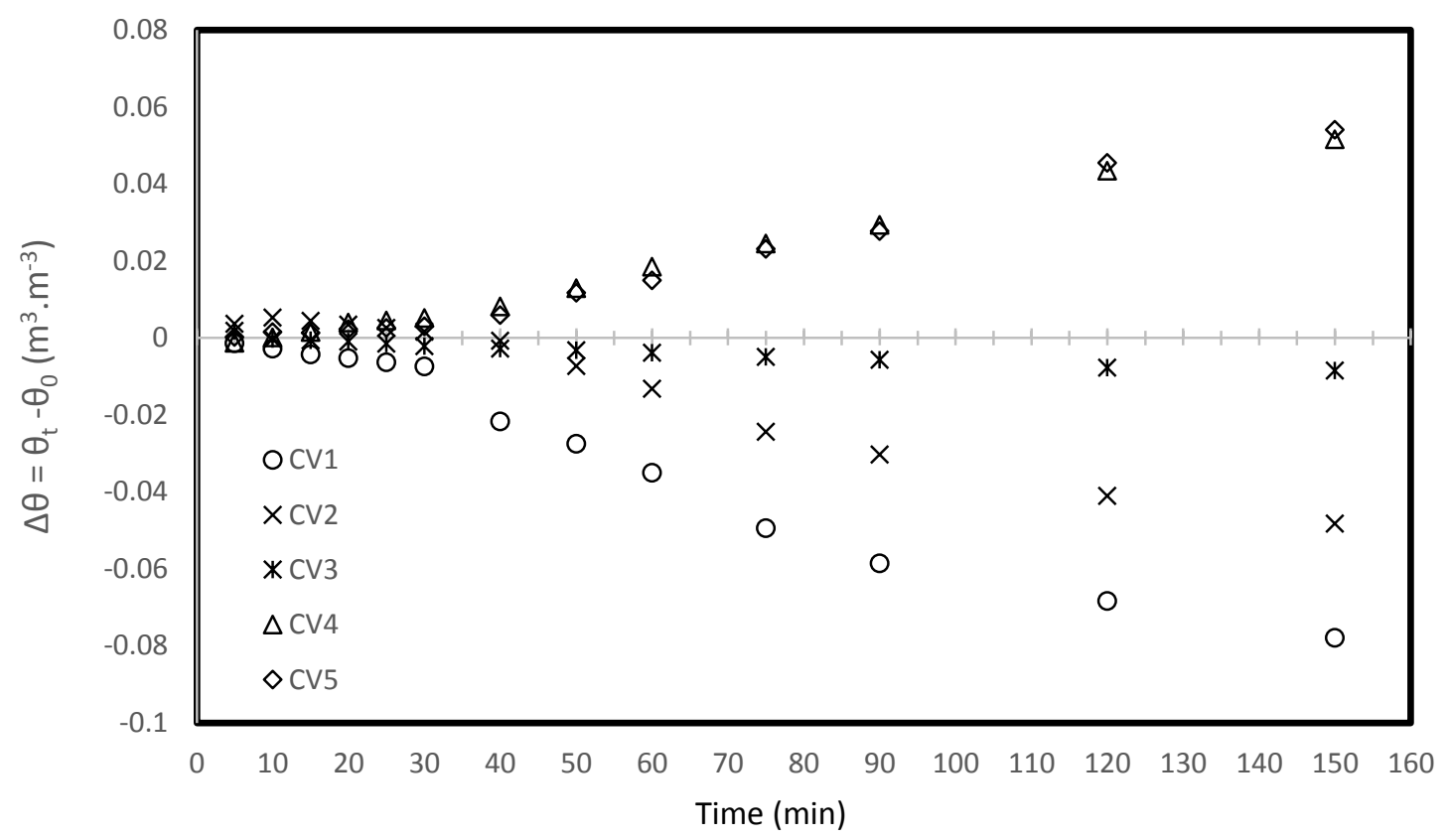

Fig. D.41 Variations of the water content differences $(\Delta \theta)$ vs. time for the case of $45-30^{\circ} \mathrm{C}$. The symbol $\Delta \theta$ is the water content difference of control volumes between time $t$ and initial time $t_{o}$. The positive value is an indication of moisture gain and the negative value is an indication of moisture loss

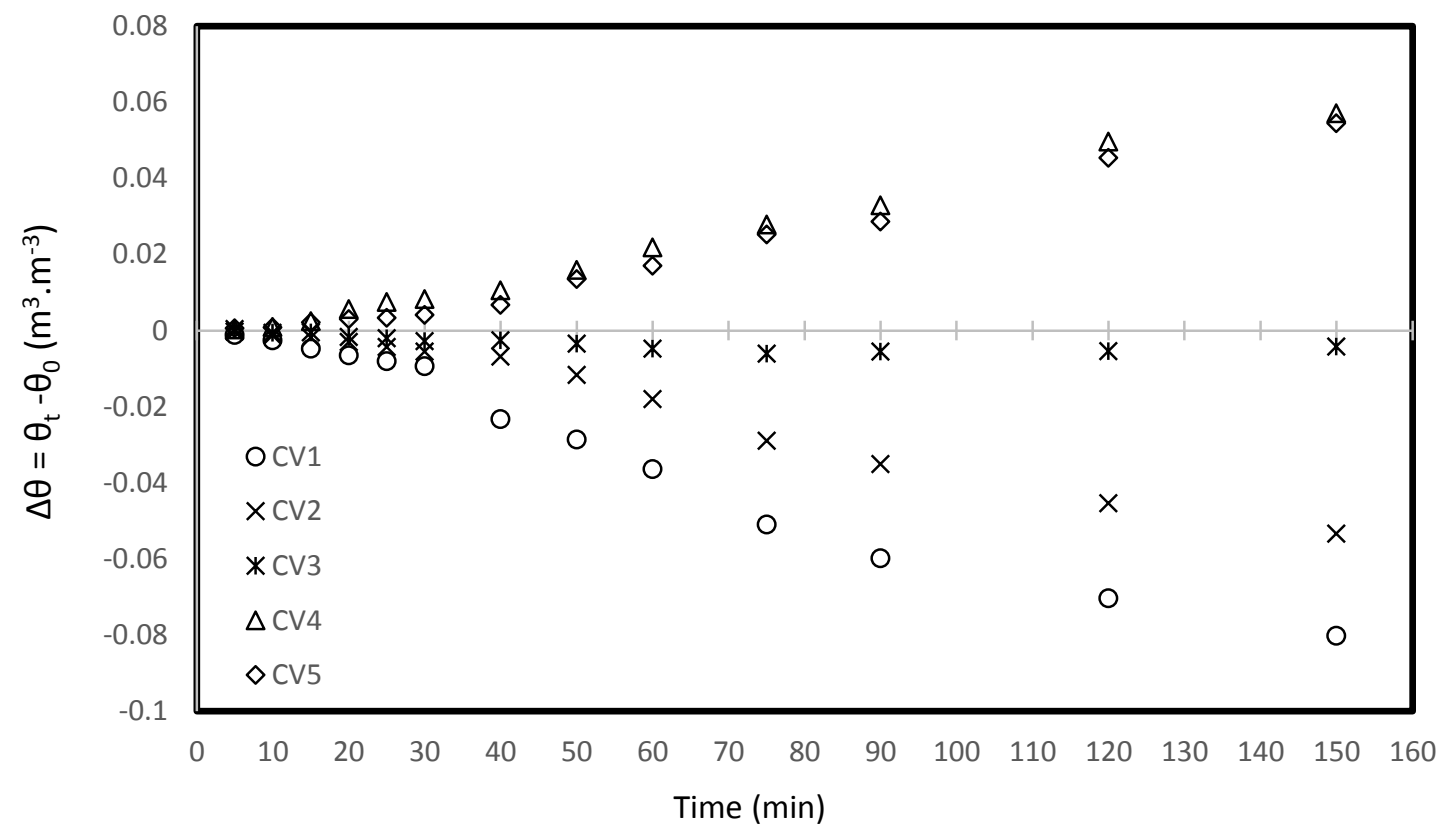

Fig. D.42 Variations of the water content differences $(\Delta \theta)$ vs. time for the case of $60-45^{\circ} \mathrm{C}$. The symbol $\Delta \theta$ is the water content difference of control volumes between time $t$ and initial time $t_{o}$. The positive value is an indication of moisture gain and the negative value is an indication of moisture loss 


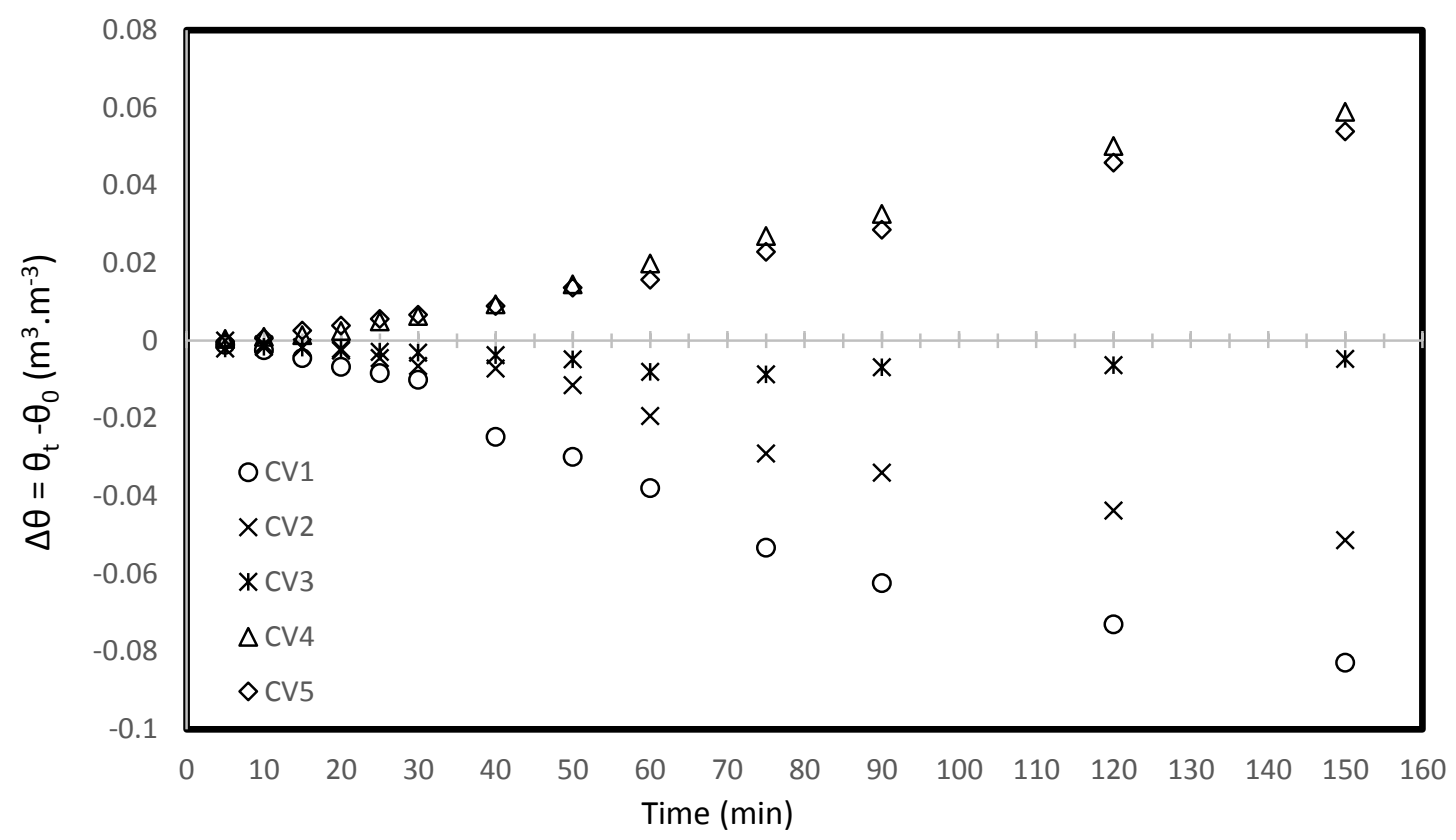

Fig. D.43 Variations of the water content differences $(\Delta \theta)$ vs. time for the case of $75-60^{\circ} \mathrm{C}$. The symbol $\Delta \theta$ is the water content difference of control volumes between time $t$ and initial time $t_{o}$. The positive value is an indication of moisture gain and the negative value is an indication of moisture loss

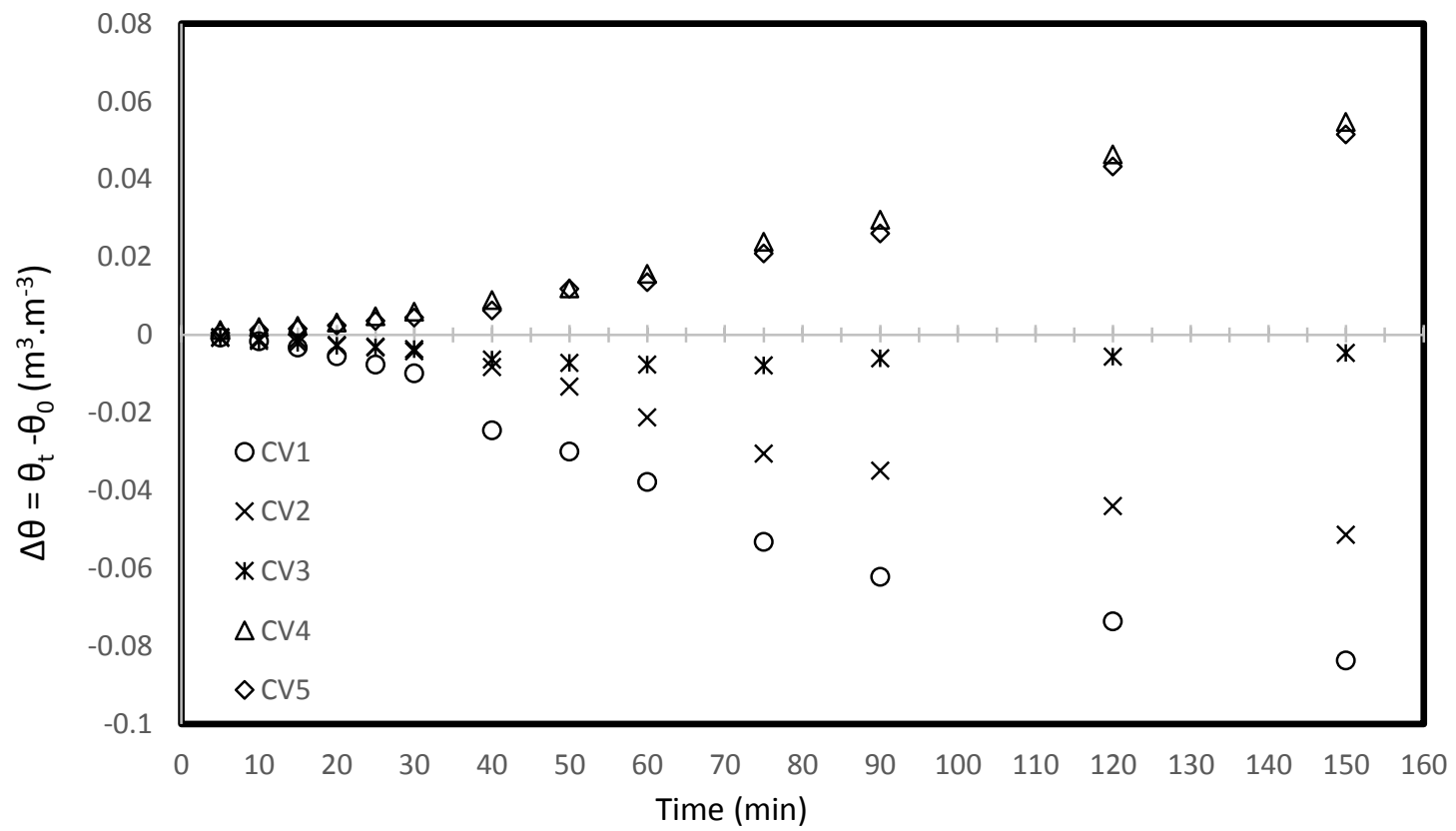

Fig. D.44 Variations of the water content differences $(\Delta \theta)$ vs. time for the case of $90-75^{\circ} \mathrm{C}$. The symbol $\Delta \theta$ is the water content difference of control volumes between time $t$ and initial time $t_{o}$. The positive value is an indication of moisture gain and the negative value is an indication of moisture loss 


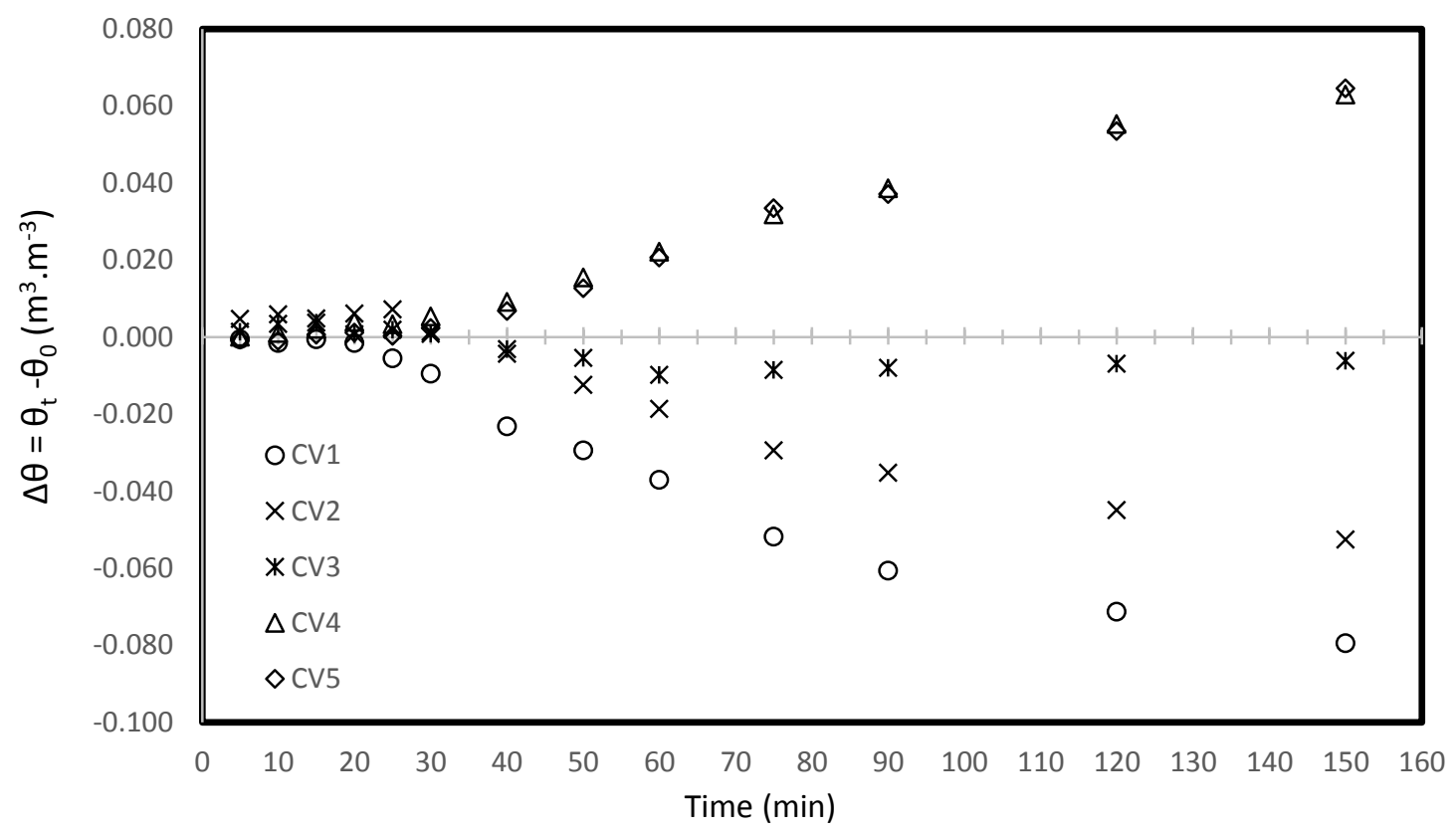

Fig. D.45 Variations of the water content differences $(\Delta \theta)$ vs. time for the case of $50-30^{\circ} \mathrm{C}$. The symbol $\Delta \theta$ is the water content difference of control volumes between time $t$ and initial time $t_{o}$. The positive value is an indication of moisture gain and the negative value is an indication of moisture loss

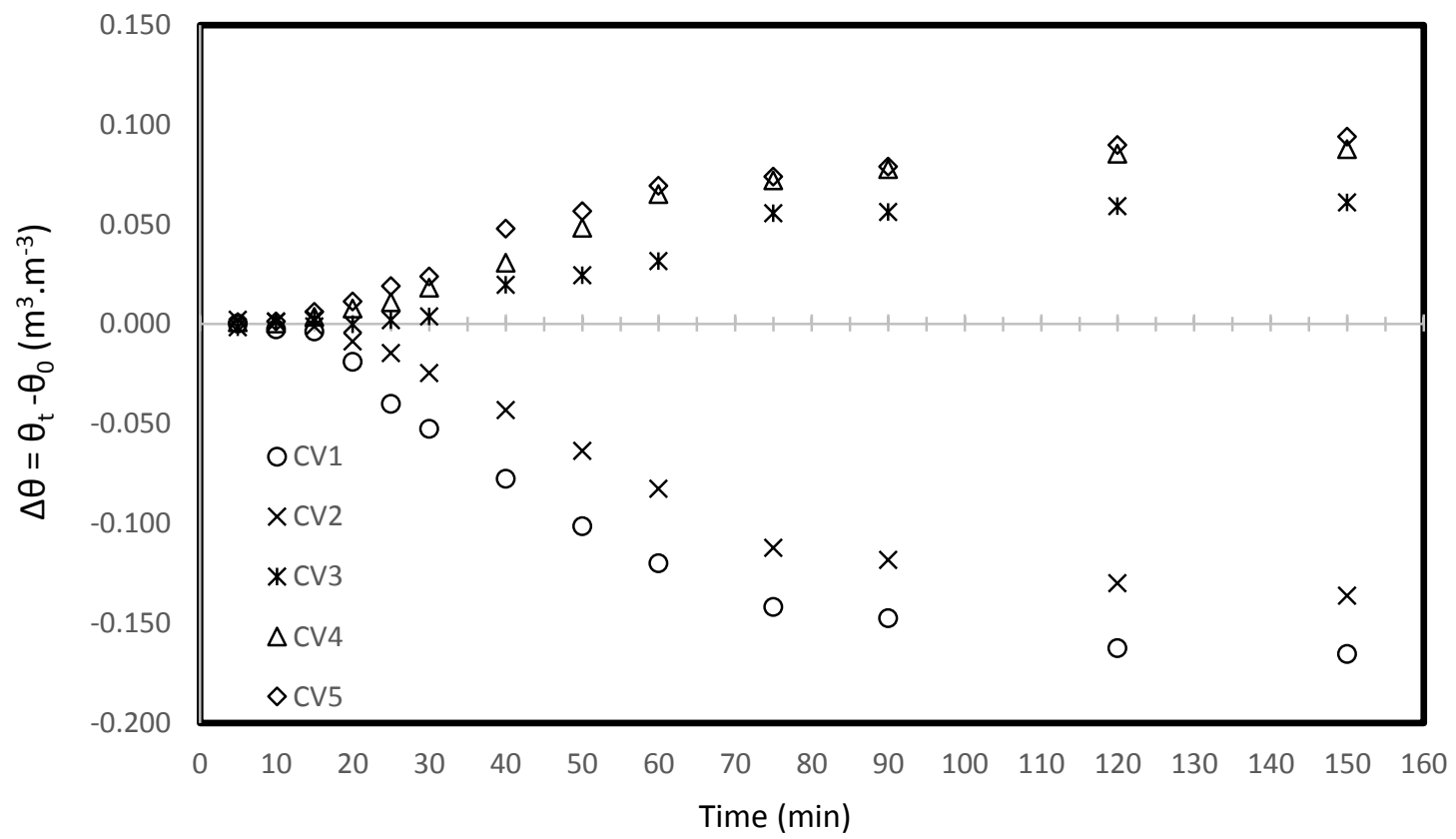

Fig. D.46 Variations of the water content differences $(\Delta \theta)$ vs. time for the case of $60-20^{\circ} \mathrm{C}$. The symbol $\Delta \theta$ is the water content difference of control volumes between time $t$ and initial time $t_{o}$. The positive value is an indication of moisture gain and the negative value is an indication of moisture loss 


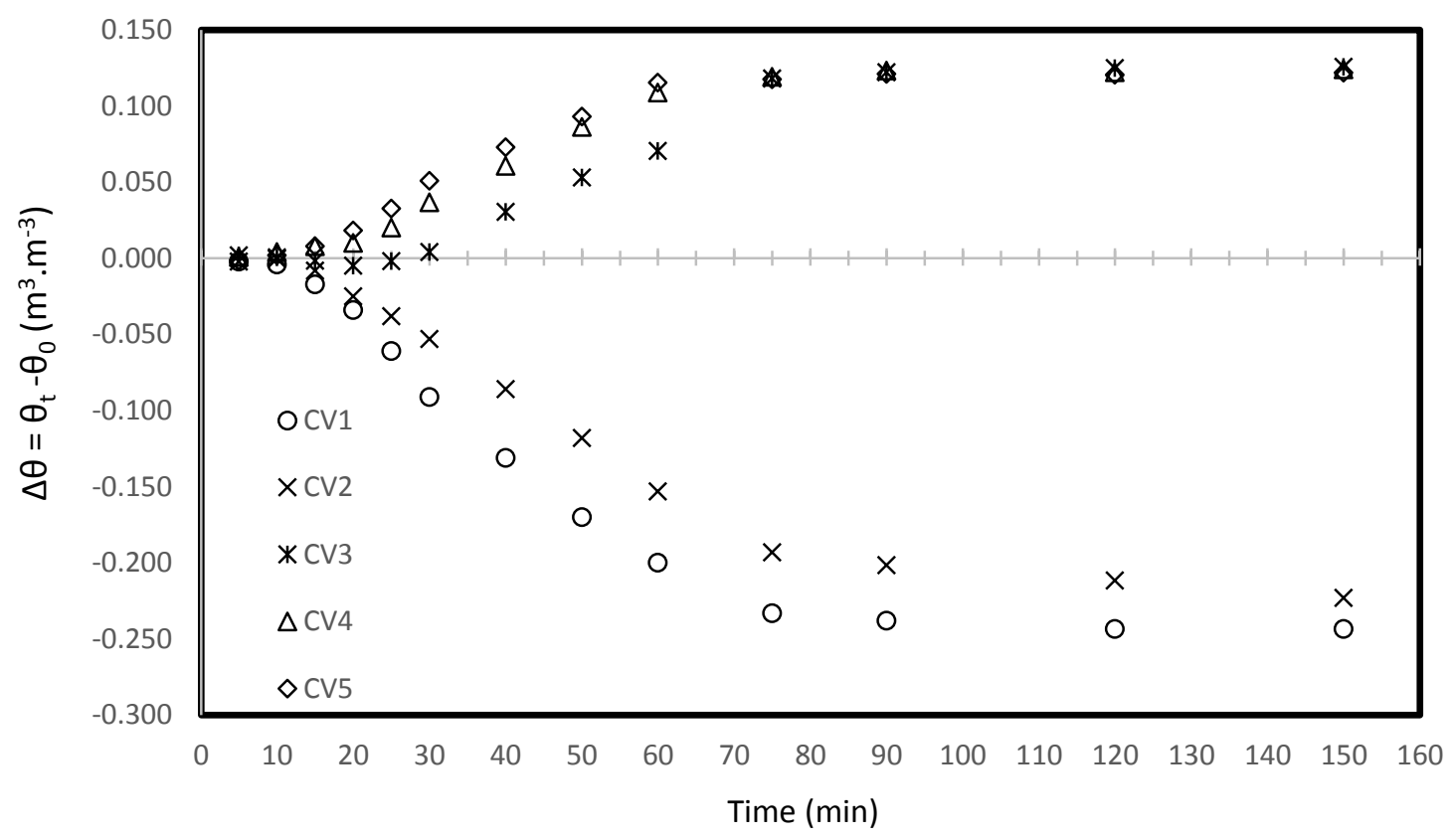

Fig. D.47 Variations of the water content differences $(\Delta \theta)$ vs. time for the case of $70-10^{\circ} \mathrm{C}$. The symbol $\Delta \theta$ is the water content difference of control volumes between time $t$ and initial time $t_{o}$. The positive value is an indication of moisture gain and the negative value is an indication of moisture loss

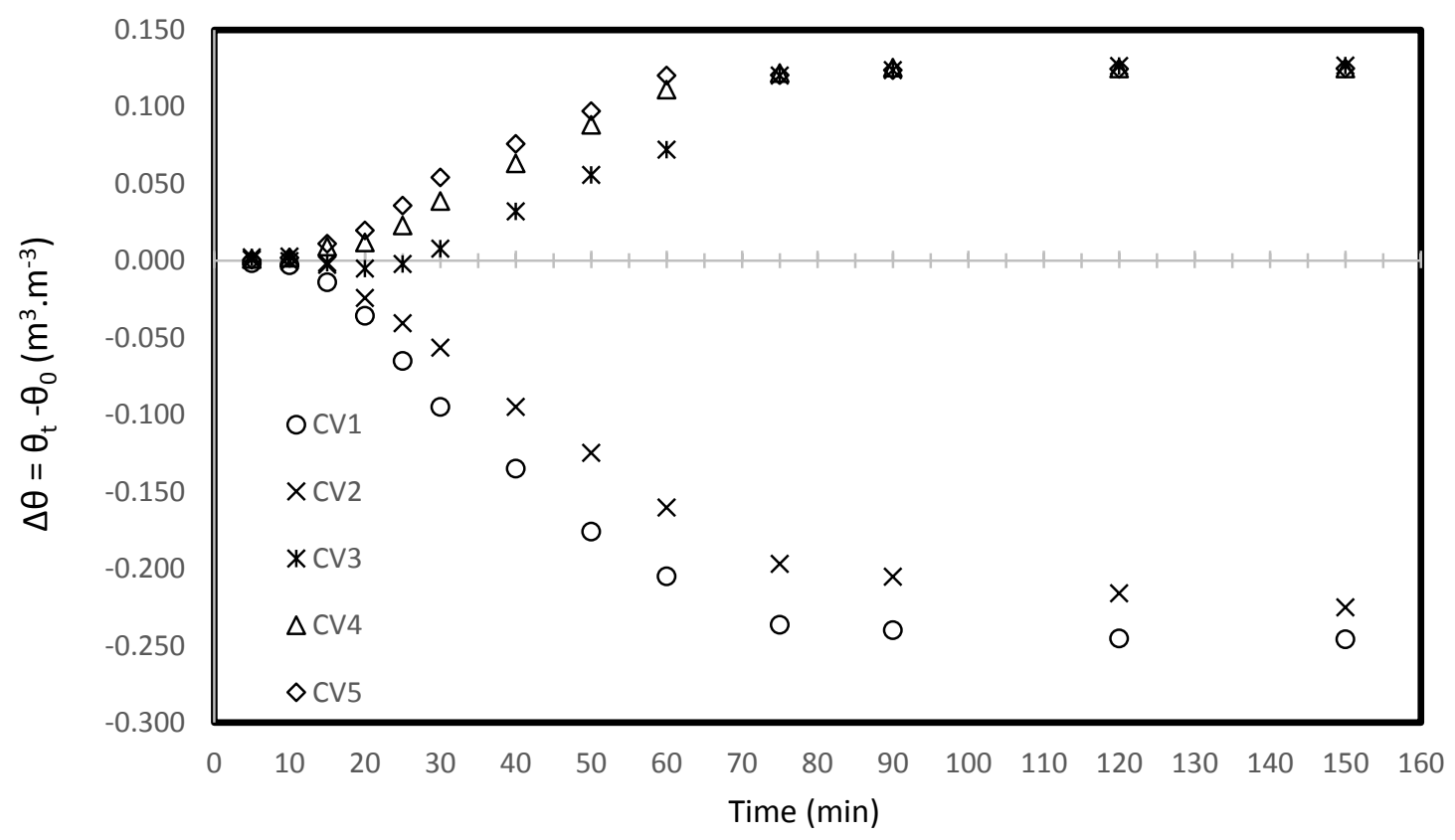

Fig. D.48 Variations of the water content differences $(\Delta \theta)$ vs. time for the case of $80-10^{\circ} \mathrm{C}$. The symbol $\Delta \theta$ is the water content difference of control volumes between time $t$ and initial time $t_{o}$. The positive value is an indication of moisture gain and the negative value is an indication of moisture loss 


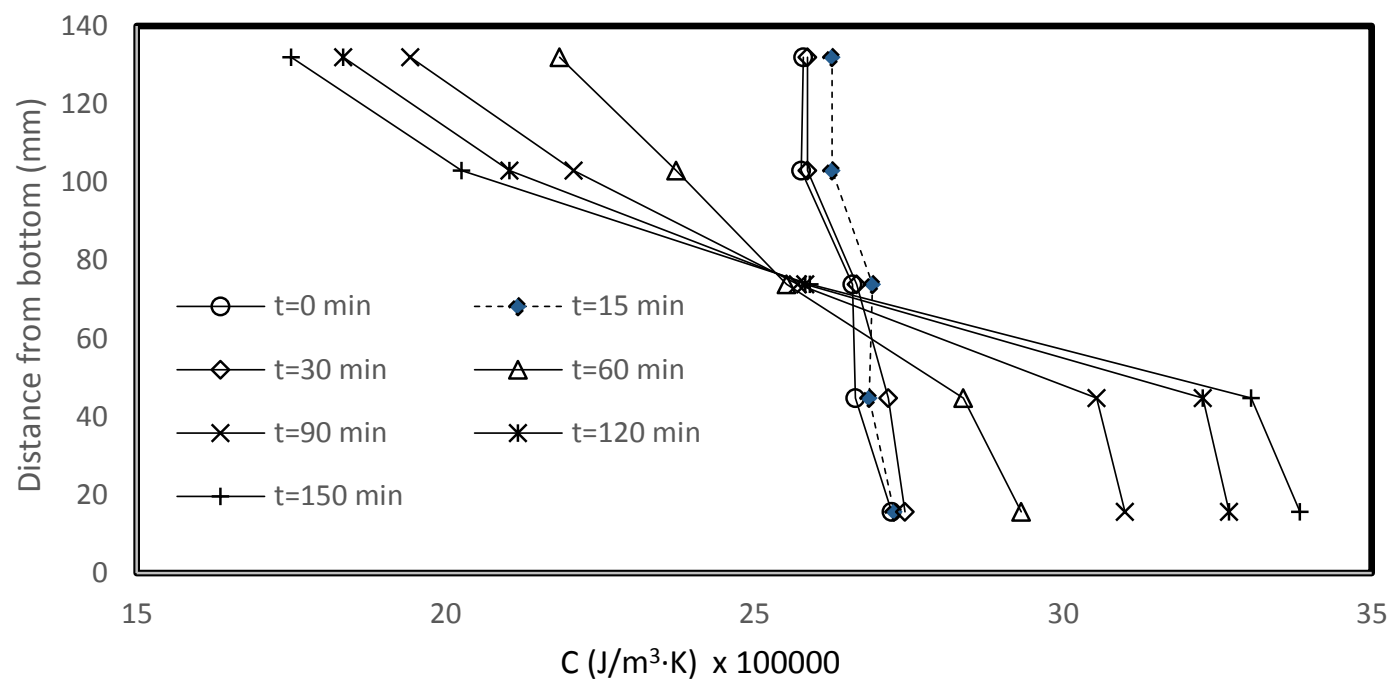

(a)

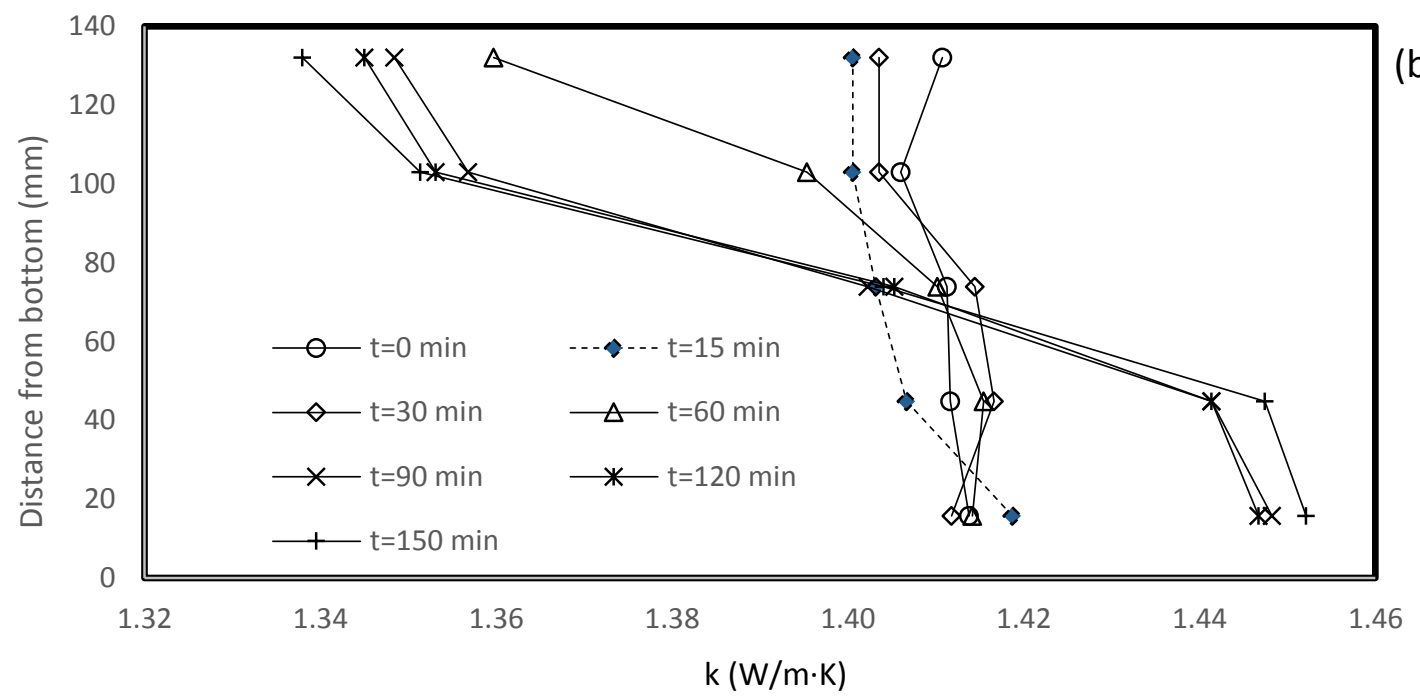

(b)

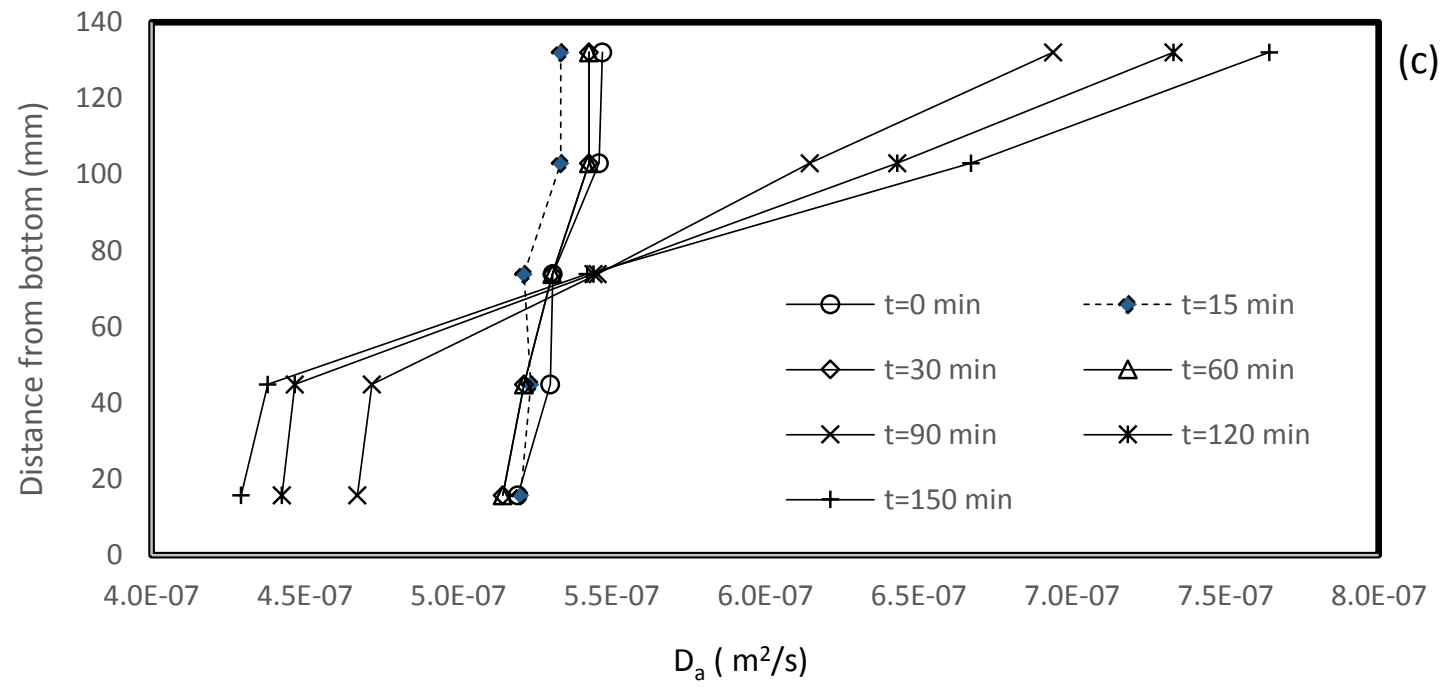

Fig. D.49 Variations of the thermal properties vs. time along the soil column for the case of 45$30^{\circ} \mathrm{C}$. a) Volumetric heat capacity b) Thermal conductivity c) Thermal diffusivity 


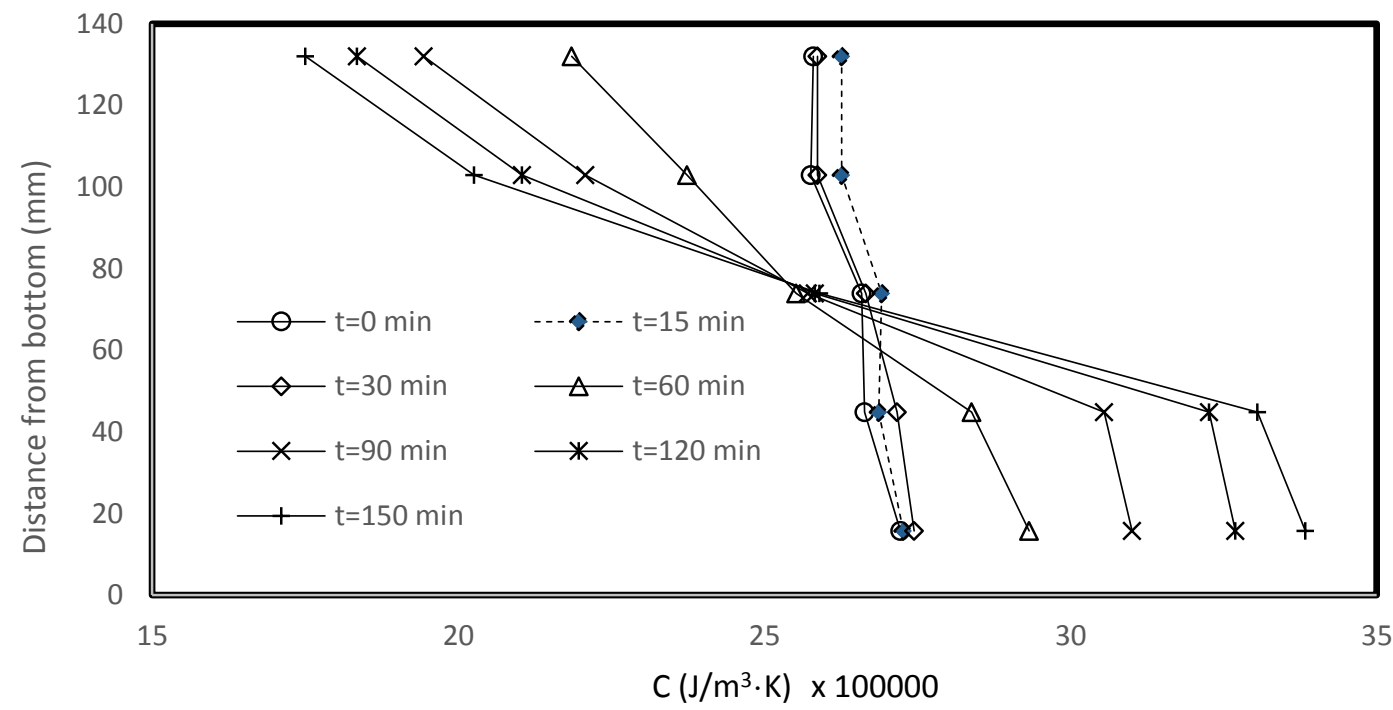

(a)

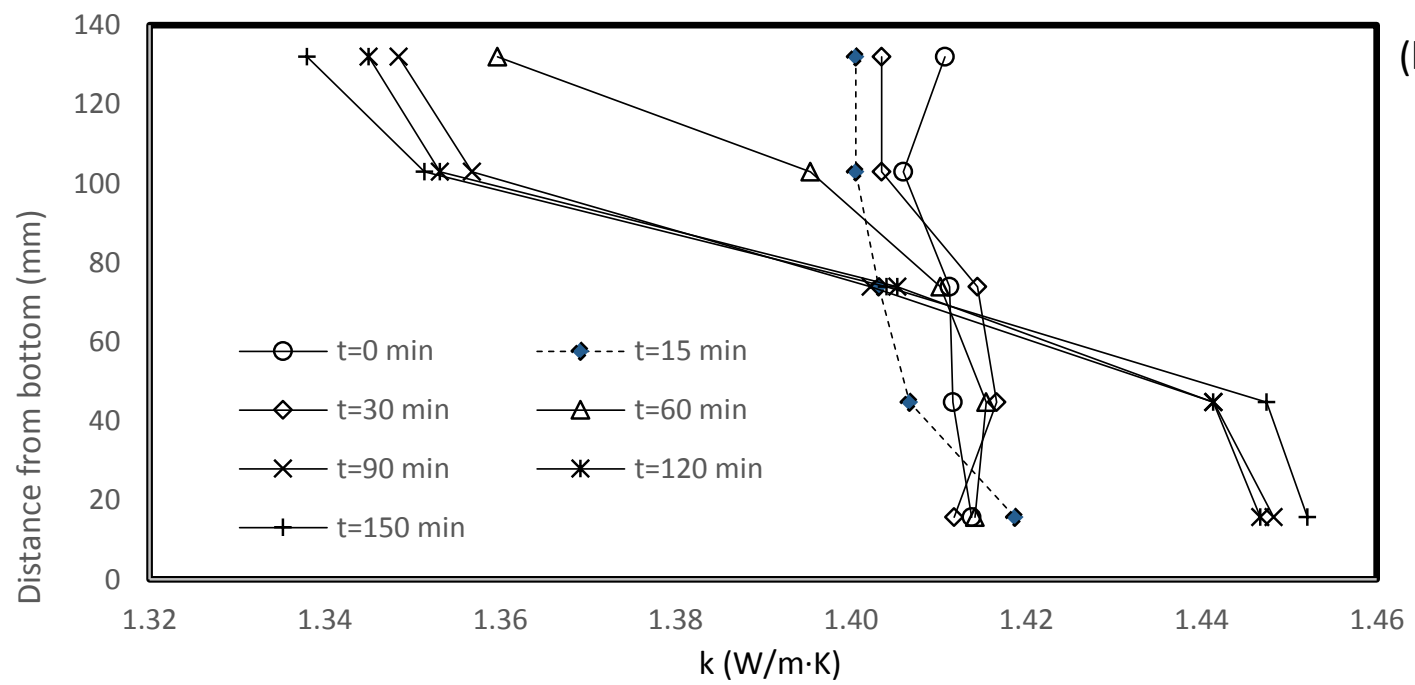

(b)

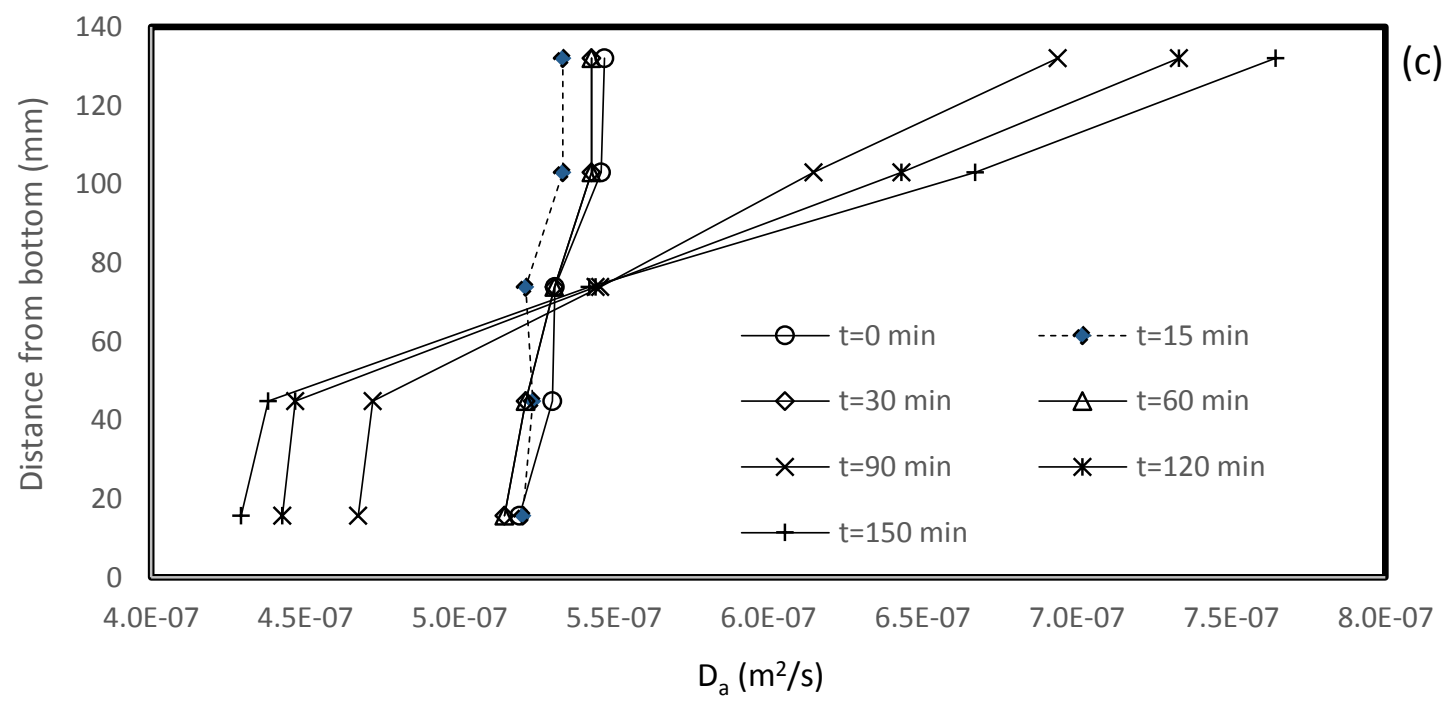

Fig. D.50 Variations of the thermal properties vs. time along the soil column for the case of 60$45^{\circ} \mathrm{C}$. a) Volumetric heat capacity b) Thermal conductivity c) Thermal diffusivity 


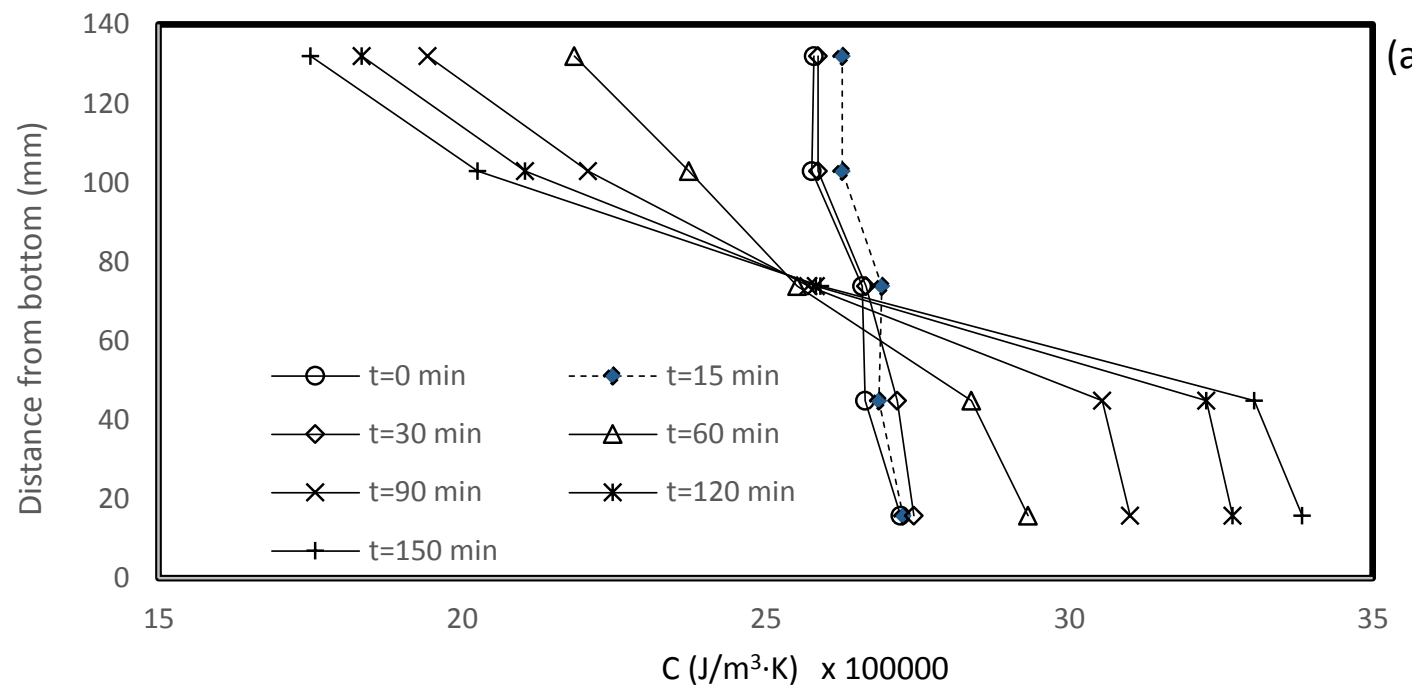

(a)
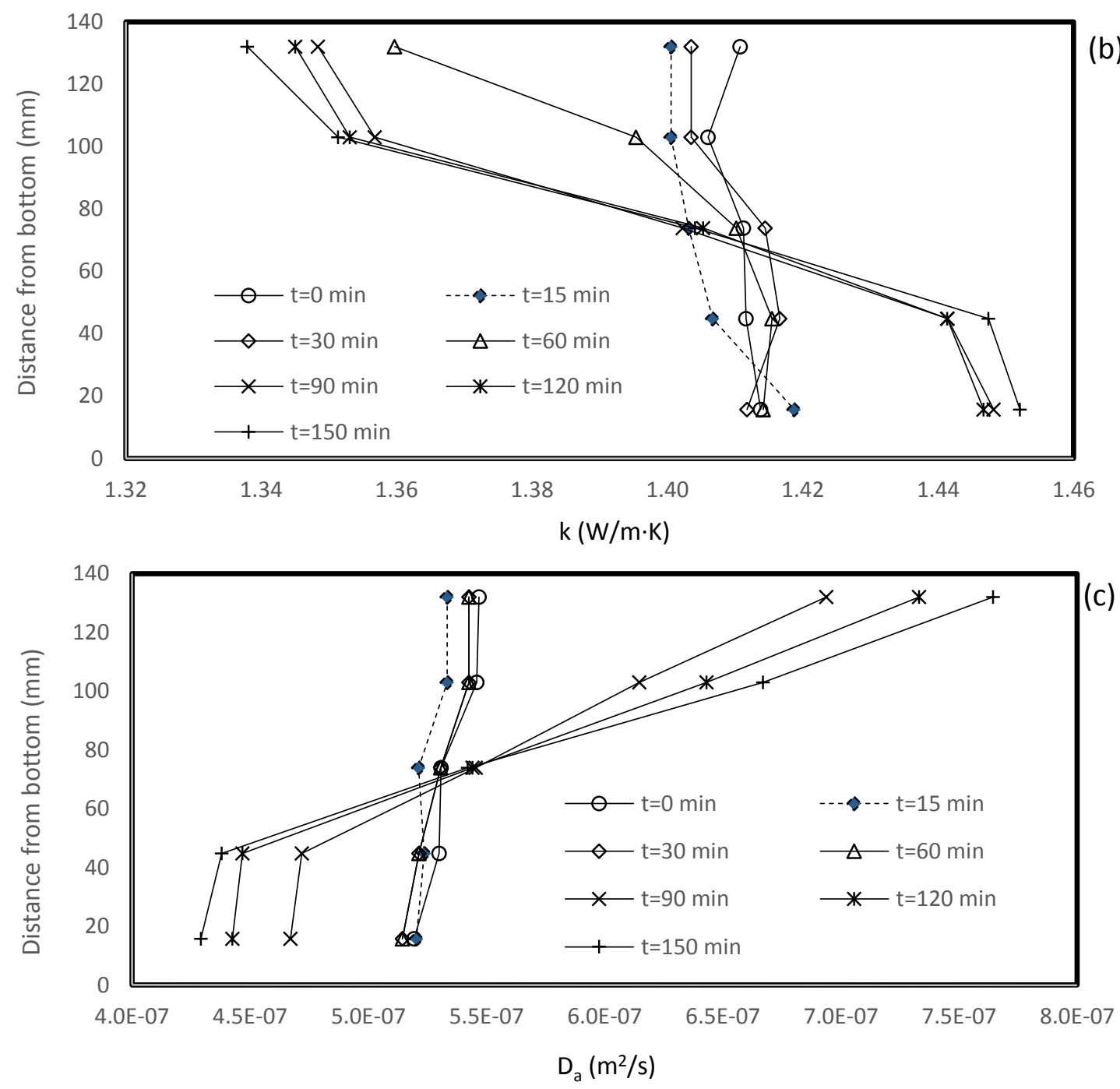

Fig. D.51 Variations of the thermal properties vs. time along the soil column for the case of 75$60^{\circ} \mathrm{C}$. a) Volumetric heat capacity b) Thermal conductivity c) Thermal diffusivity 


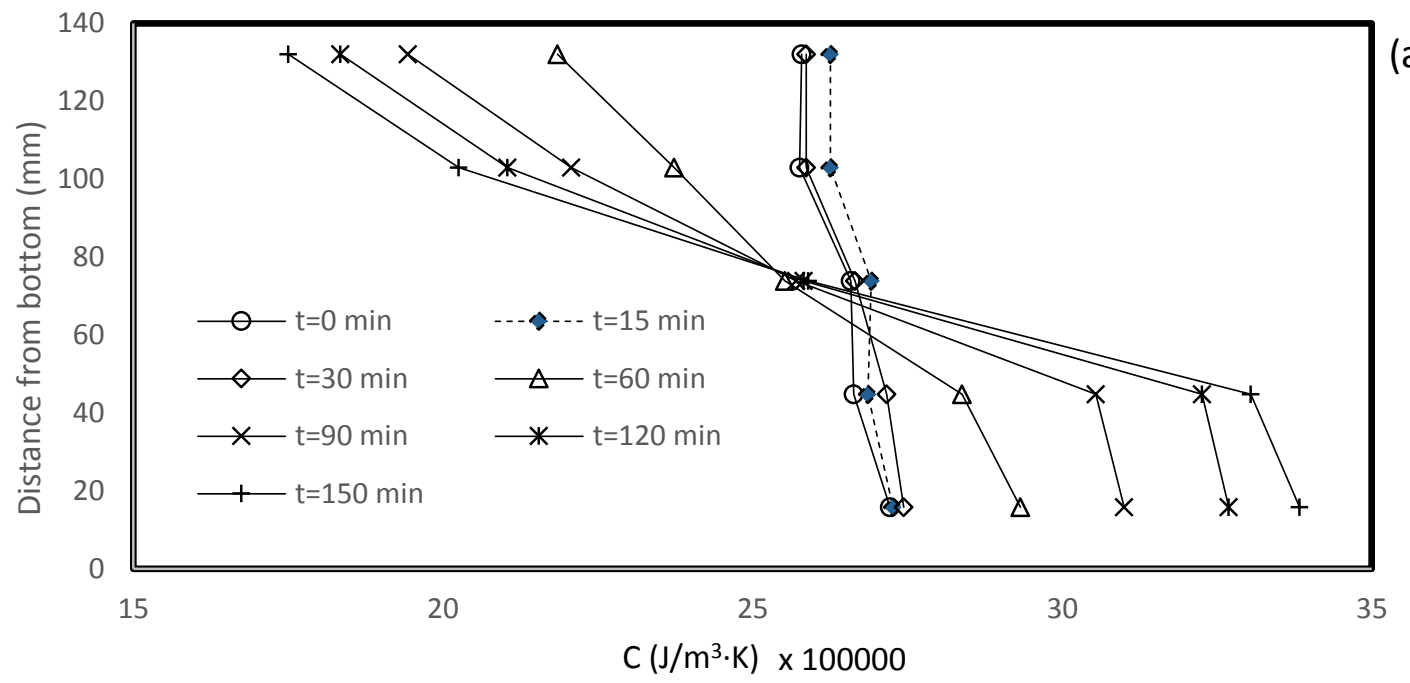

(a)
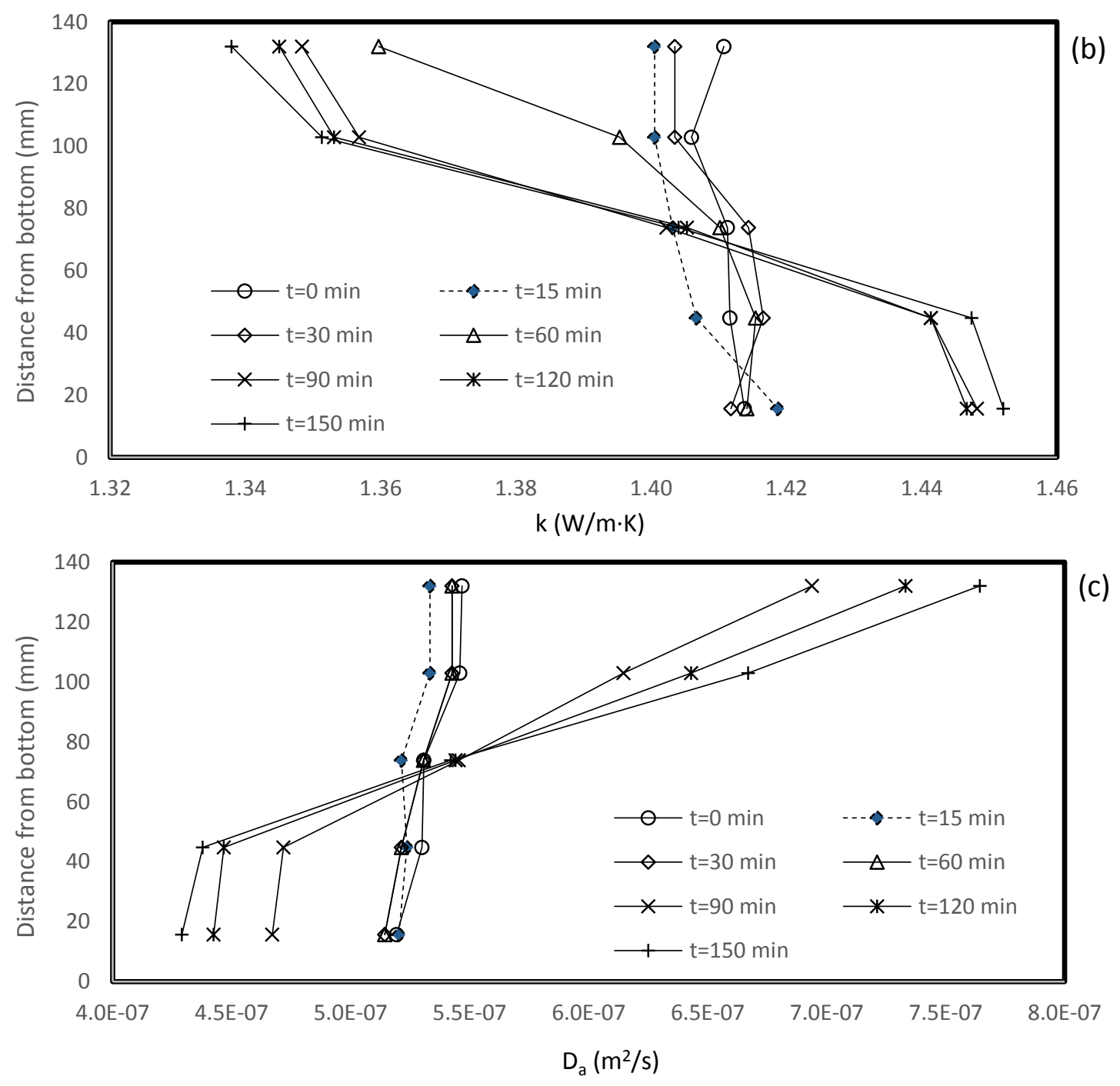

Fig. D.52 Variations of the thermal properties vs. time along the soil column for the case of 90$75^{\circ} \mathrm{C}$. a) Volumetric heat capacity b) Thermal conductivity c) Thermal diffusivity 


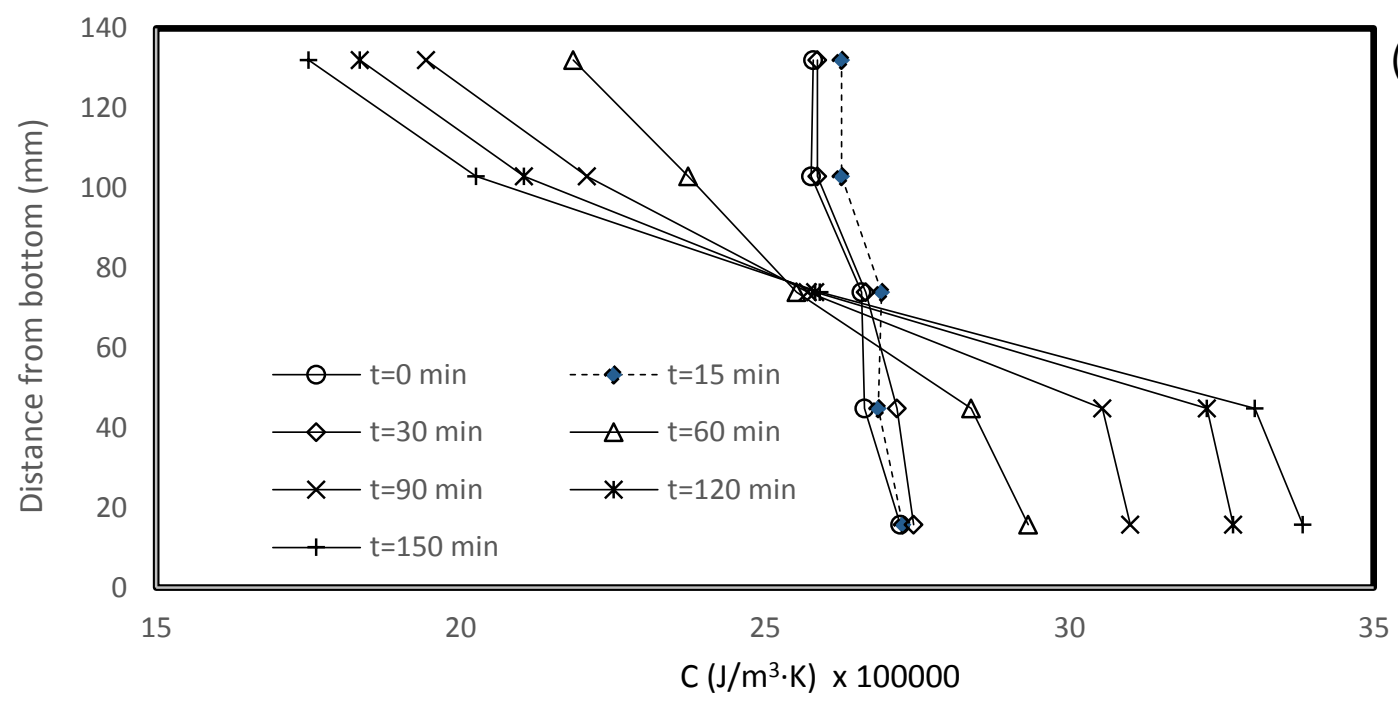

(a)
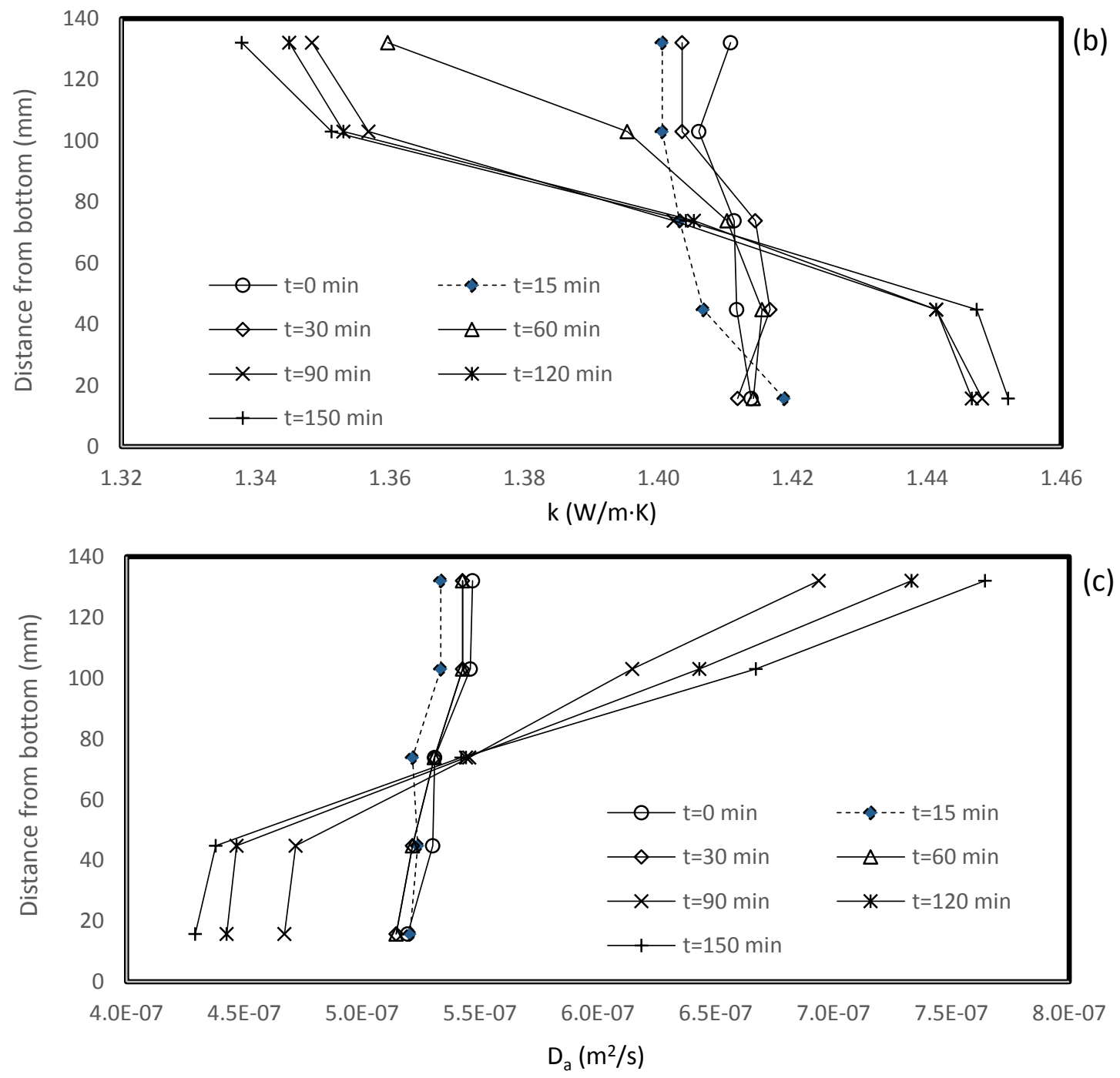

Fig. D.53 Variations of the thermal properties vs. time along the soil column for the case of 50$30^{\circ} \mathrm{C}$. a) Volumetric heat capacity b) Thermal conductivity c) Thermal diffusivity 


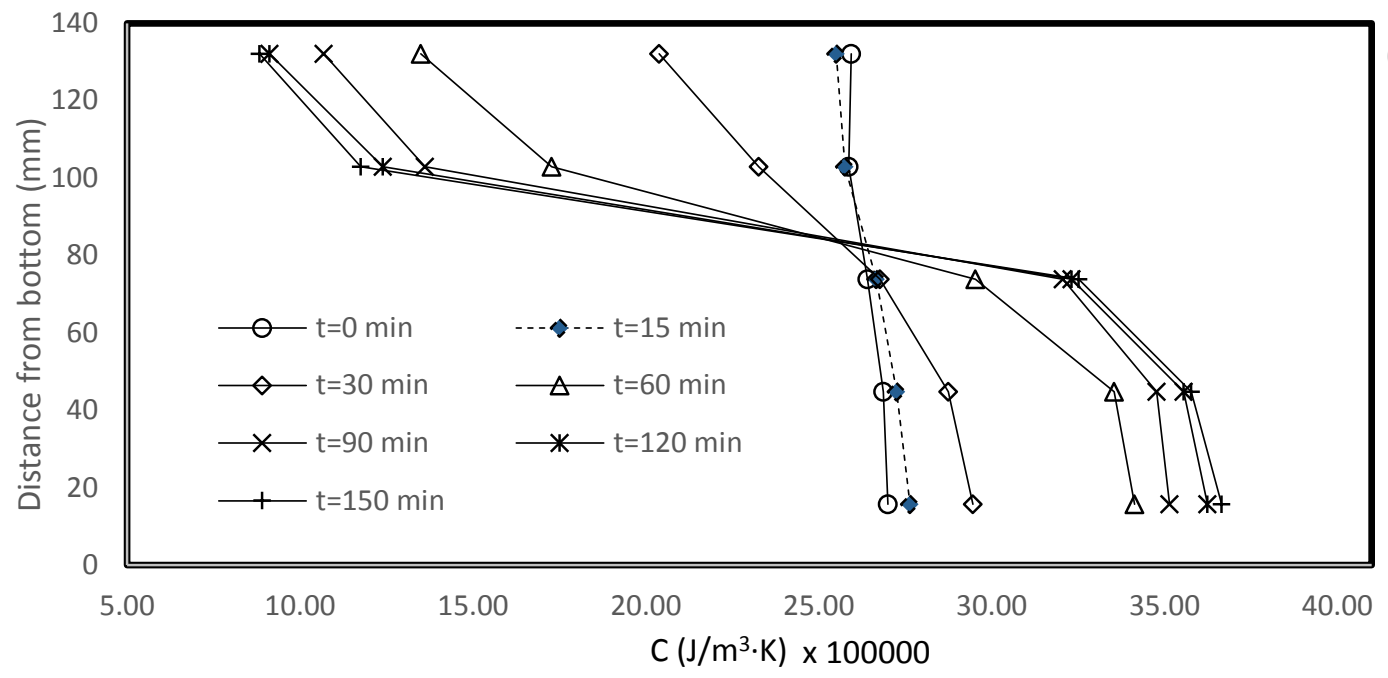

(a)
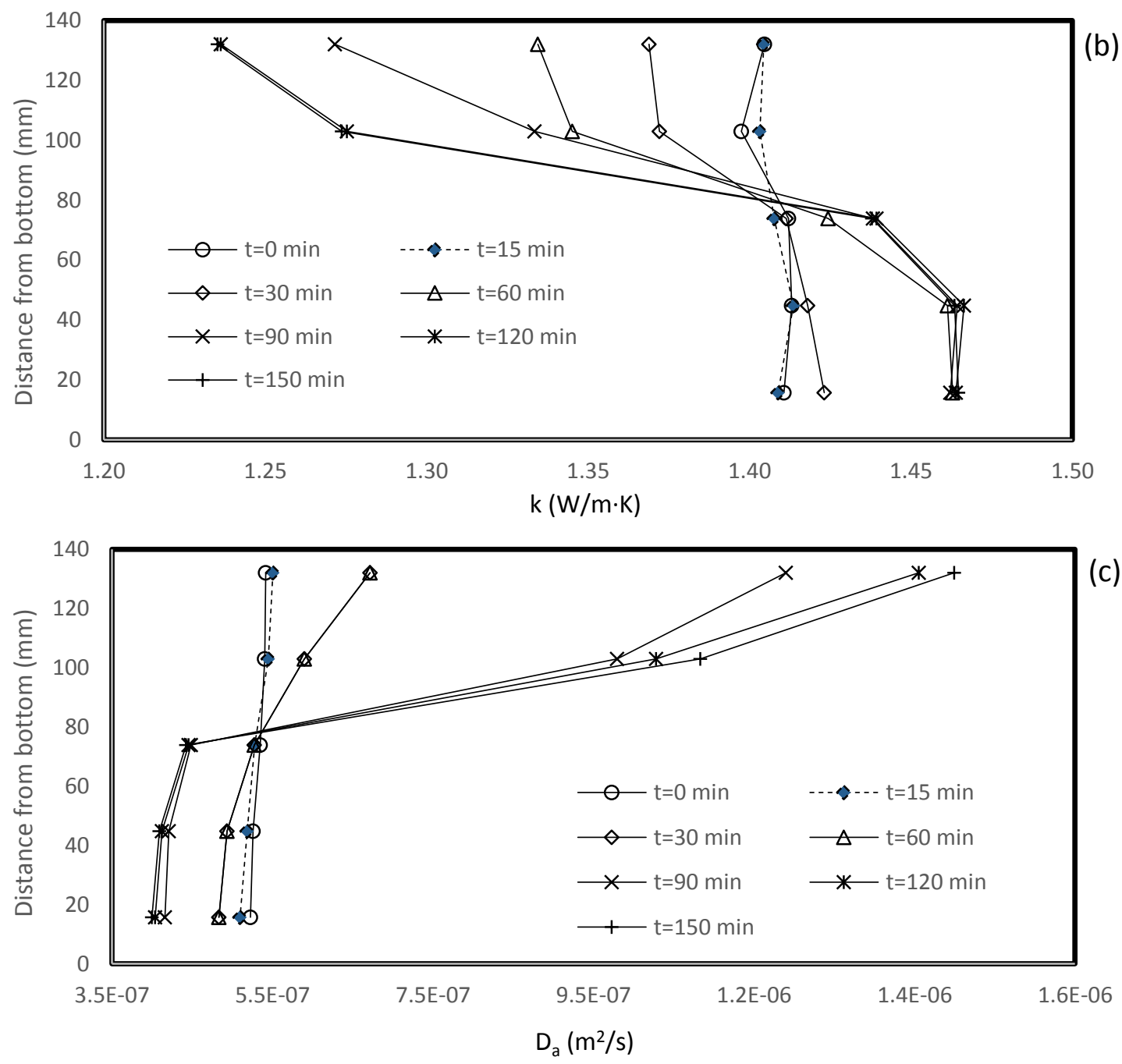

Fig. D.54 Variations of the thermal properties vs. time along the soil column for the case of 60$20^{\circ} \mathrm{C}$. a) Volumetric heat capacity b) Thermal conductivity c) Thermal diffusivity 


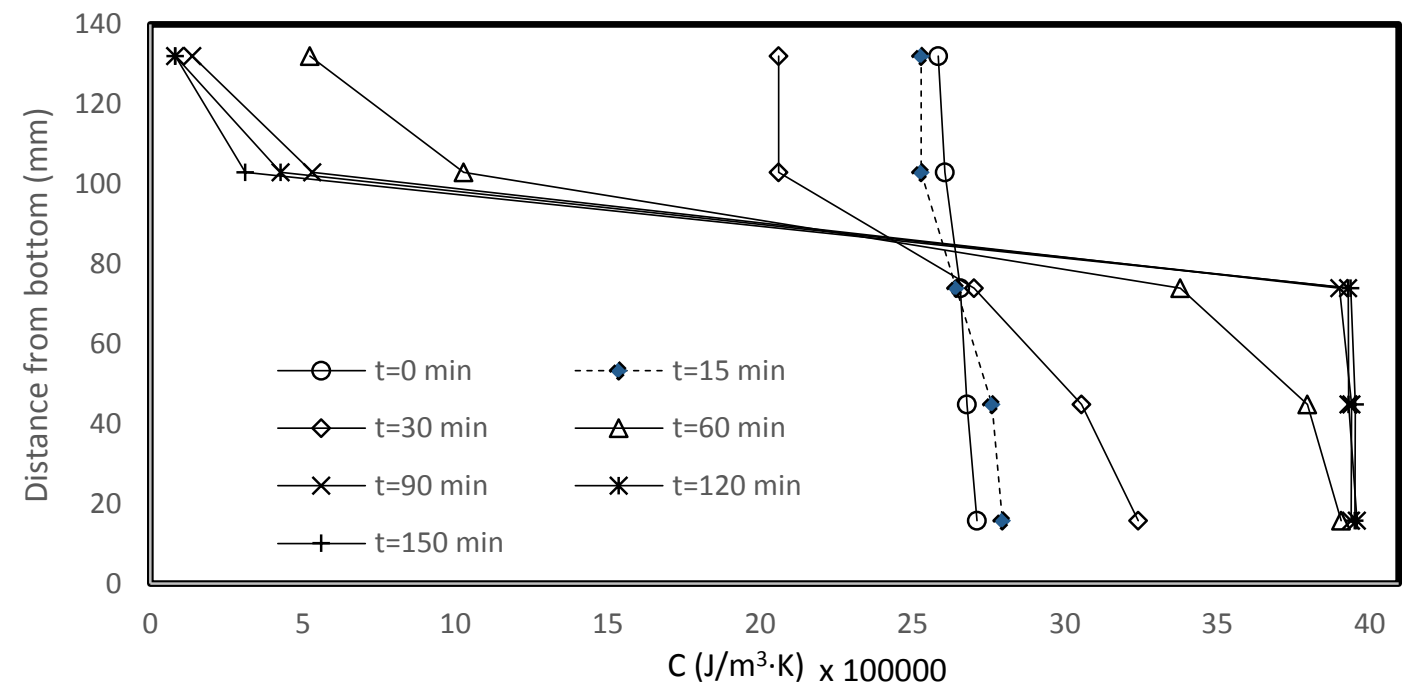

(a)

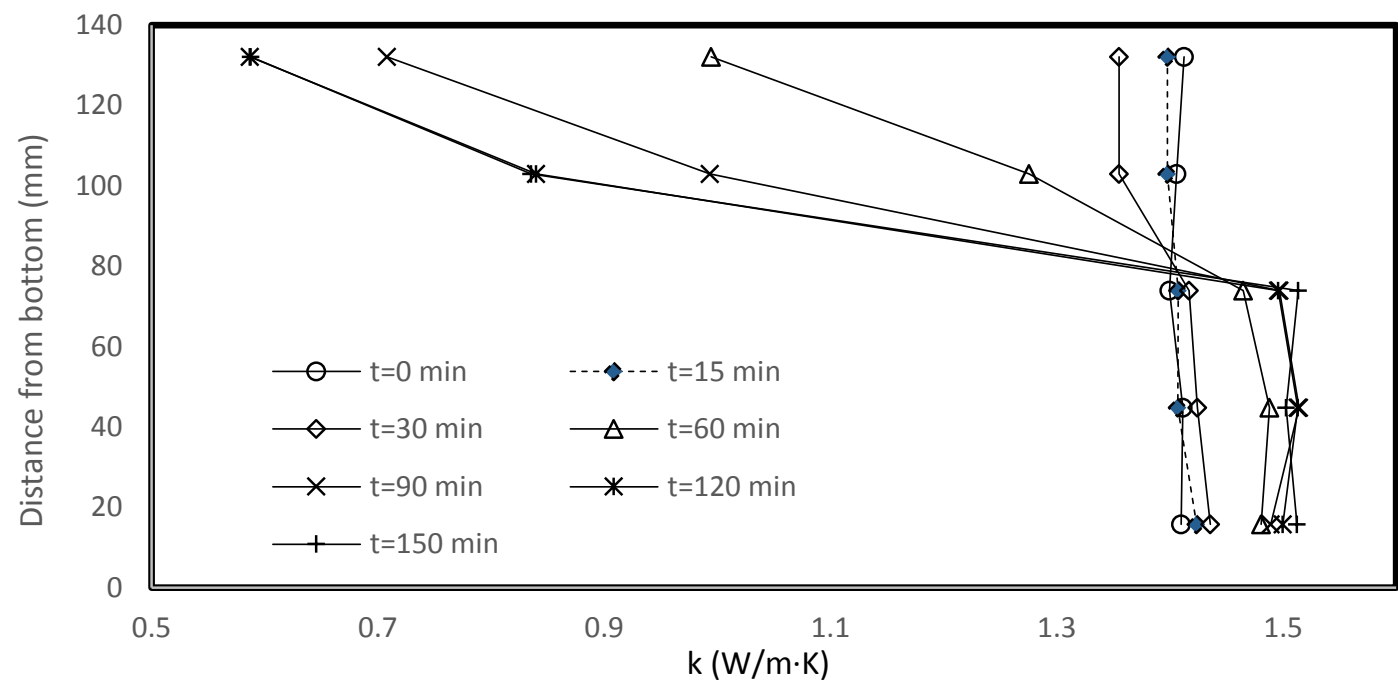

(b)

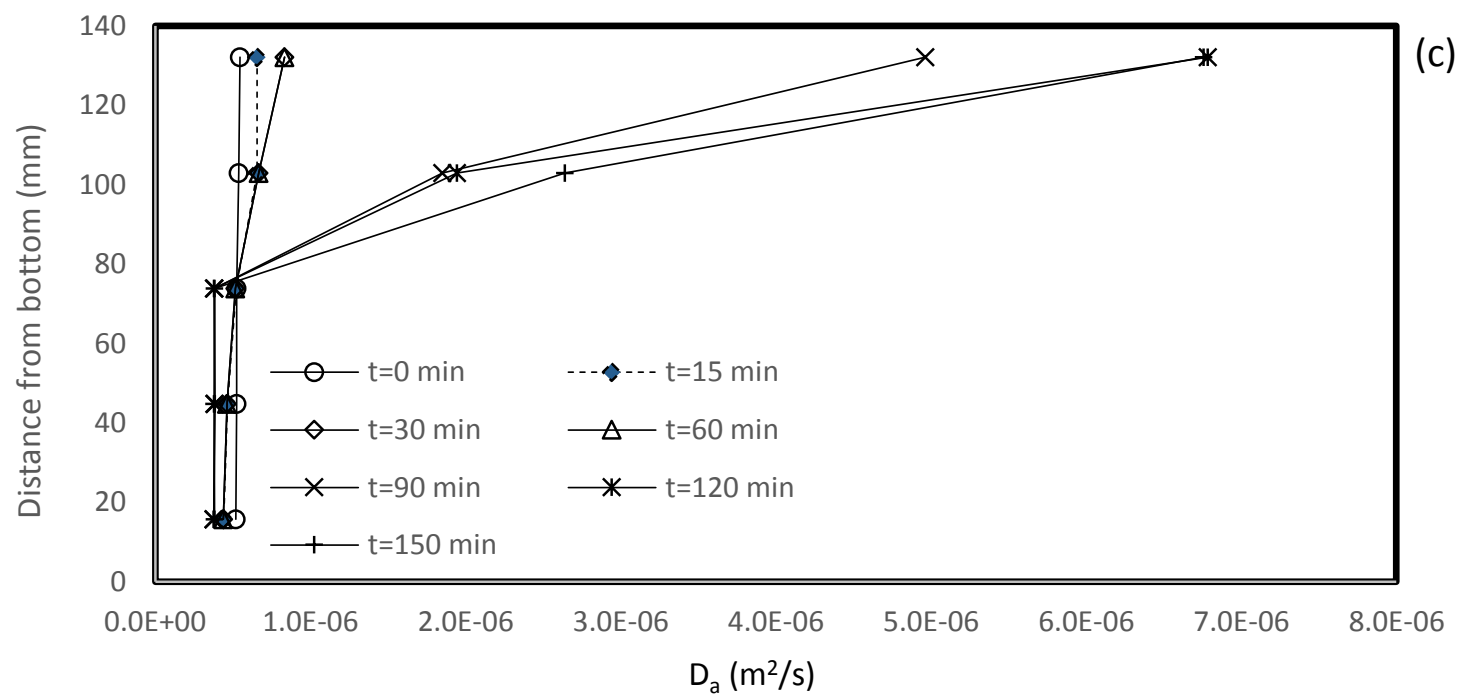

Fig. D.55 Variations of the thermal properties vs. time along the soil column for the case of 70$10^{\circ} \mathrm{C}$. a) Volumetric heat capacity b) Thermal conductivity c) Thermal diffusivity 


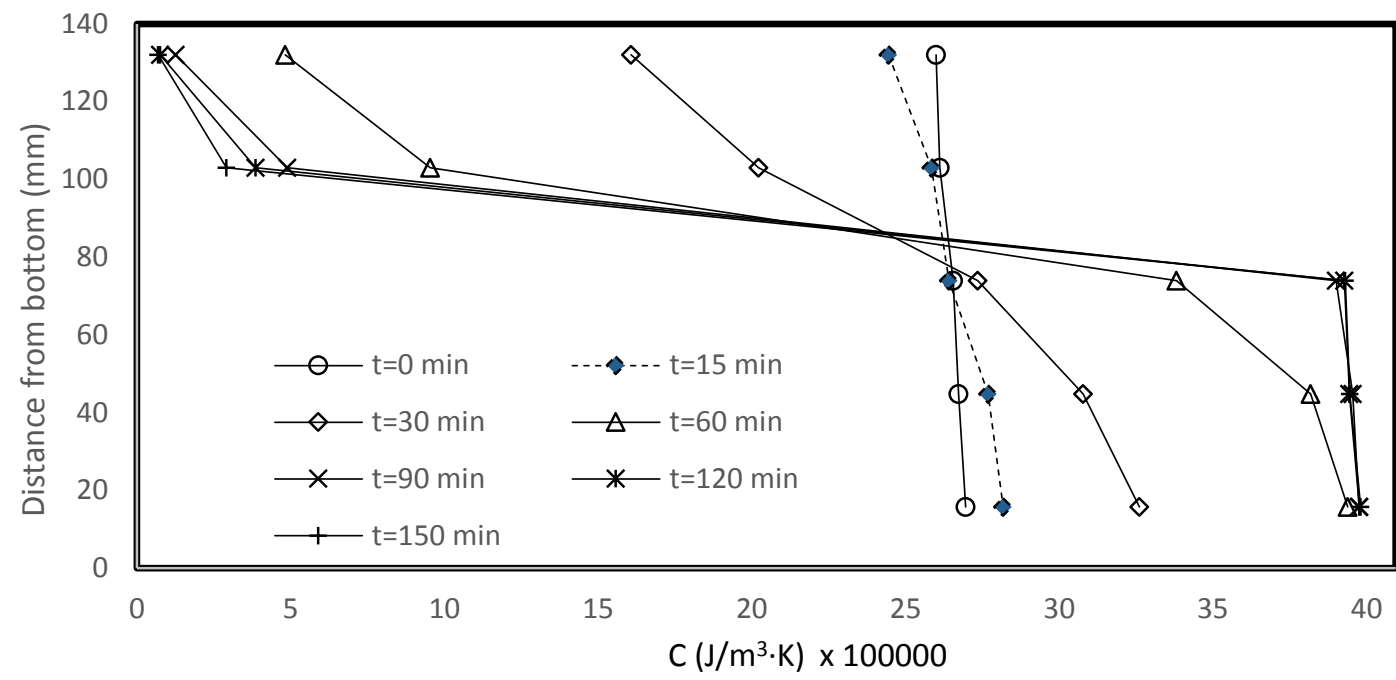

(a)

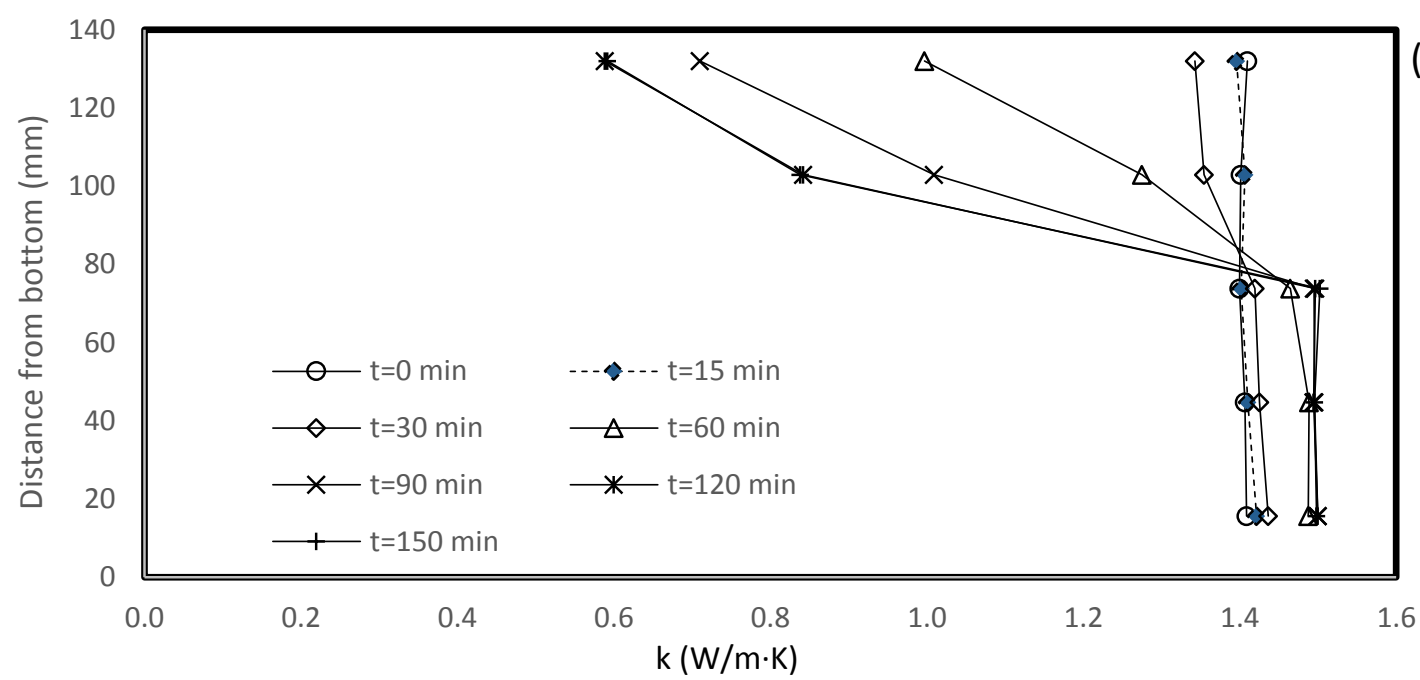

(b)

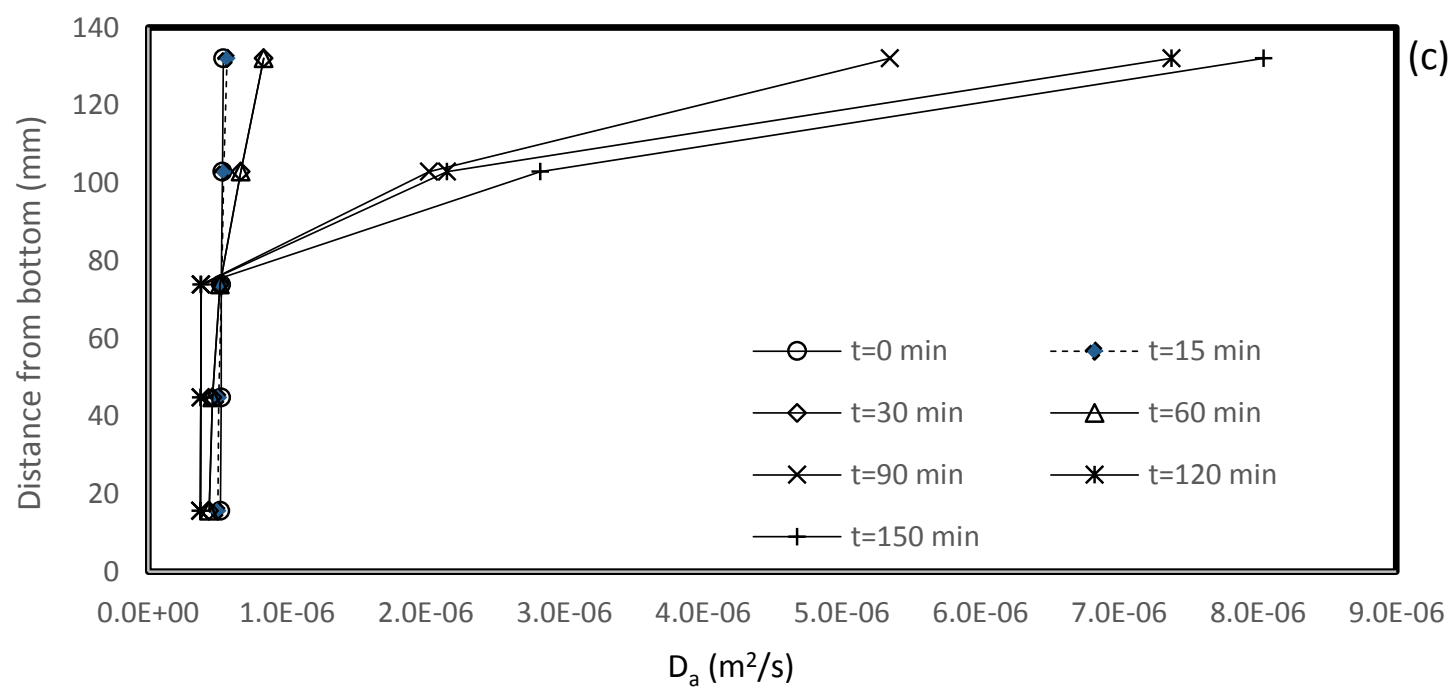

Fig. D.56 Variations of the thermal properties vs. time along the soil column for the case of 80$10^{\circ} \mathrm{C}$. a) Volumetric heat capacity b) Thermal conductivity c) Thermal diffusivity 


\section{REFERENCES}

Abramowitz, M., and Stegun. I., Hand book of mathematical functions. Dover publications, NewYork, 1972.

Baker, J. M. and Lascano, R.J., The spatial sensitivity of time-domain reflectometry. Soil Science Vol. 147, pp. 378-384, 1989.

Baker, J.M., Allmaras, R.R., System for automating and multiplexing soil moisture measurement by time-domain reflectometry. Soil Sci. Soc. Am. J. Vol. 54, pp. 1-6, 1990.

Bristow, K.L., Campbell, G.S., and Calissendorff, K., Test of a heat-pulse probe for measuring changes in soil water content. Soil Sci. Soc. Am. J. Vol. 57, pp. 930-934, 1993.

Bristow, K. L., Kluitenberg G. J., and Horton, R., Measurement of soil thermal properties with a dual-probe heat-pulse technique, National Soil Tilth Laboratory, Ames, IA. pp. 1298-1293, 1994.

Bristow, K. L., Bilskie, J. R. Kluitenberg, G.J., and Horton, R., Comparison of techniques for extracting soil thermal properties from dual-probe heat-pulse data. Soil Sci. Vol. 160, pp. 1-7, 1995.

Cary, J. W., Soil Moisture Transport due to Thermal Gradients: Practical Aspects, Vol. 30, pp. 428-433, 1966.

Campbell, G.S., C. Calissendorff, and J.H. Williams, Probe for measuring soil specific heat using a heat-pulse method. Soil Sci. Soc. Am. J. Vol. 55, pp. 291-293, 1991.

Campbell, G.S., Jungbauer, J. D., Bidlake, W. R., and Hungerford, R. D., Predicting the effect of temperature on soil thermal conductivity, Soil Science, Vol. 158, pp. 307-313, 1994.

Campbell Scientific, Instruction manual for TDR probes, Campbell Scientific Canada Corporation, Edmonton, Alberta, Canada, 2013.

de Vries, D. A., A nonstationry method for determining thermal conductivity of soil in situ. Soil Sci. Vol. 73, pp. 83-89, 1952a. 
de Vries, D.A, A nonstationary method for determining thermal conductivity of soil in situ, Soil Science Vol. 73, pp. 83-89, 1952 b.

de Vries, D. A., Simultaneous transfer of heat and moisture in porous media, Transactions of American Geophysical Union, Vol. 39, pp. 909-915, 1958.

de Vries, D. A., Thermal properties of soils in physics of plant environment, North-Holland Publishing Company, Amsterdam pp. 210-235, 1963.

de Vries, D. A., Heat transfer in soils, in heat and mass transfer, in the biosphere, transfer processes in Plant Environment, pp. 4-6, 1974.

de Vries, D. A., The theory of heat and moisture transfer in porous media revisited. International Journal of Heat and Mass Transfer, Vol. 30, pp. 1343-1350, 1987.

Evett, S. R., Tolk, J. A., and Howell, T. A., Time domain reflectometry laboratory calibration in travel time, bulk electrical conductivity, and effective frequency, Vadose Zone Journal Vol. 4, pp. 1020-1029, 2005.

Farouki, O. T., Thermal properties of soils, CRREL Monograph Vol. 81, pp. 1-4, 1981.

Fellner-Feldegg, H., The measurement of dielectrics in the time domain. J. Phys. Chem. Vol. 73, pp. 616-623, 1969.

Heitman, J. L., Horton, R., Ren, T. and Ochsner, T. E., An improved approach for measurement of coupled heat and water transfer in soil cells, Journal of Solid Physics Vol. 71, 2007.

Horton, R., Ren, T., Ju, Z. and Gong, Y., Comparing heat-pulse and time domain reflectometry soil water contents from thermo-time domain reflectometry probes, Published in Vadose Zone Journal Vol. 4, pp. 1080-1086, 2005.

Hugh, W. Coleman, W. Glenn Steele, Experimentation, validation and uncertainty analysis for engineers, $3^{\text {rd }}$ E. Wiley \& Sons Inc., pp. 5-10. 2009.

Incropera, de Witt, Bergman, Lavine, Introduction to heat transfer, $5^{\text {th }}$ edition. pp. 535-544, 2007. 
Johansen, O. Thermal coqductivity of soils, Ph.D. thesis, Trondheim, Norwlpy (CRREL Draft Translation 637 1977) ADA 044002, 1975.

Keyhani, A. and Wulfsohn, D., Sensitivity of soil coupled heat and mass transfer governing equations to hydraulic and thermal conductivities, J. Agric. Sci. Technol. Vol. 3 pp. 227-235, 2001.

Kline, S. J., and McClintock, F. A., Describing uncertainties in single-sample experiments, Mechanical Engineering, Vol. 75, No. 1, 1953.

Kluitenberg, G. j., Ham, J. M., and Bristow, K. L, Error analysis of the heat pulse method for measuring soil volumetric heat capacity. Soil Sci. Soc. Am. J. Vol. 57, pp. 1444-1451, 1993.

Kluitenberg, G. J., Bristow, K. L., and Das, B. S., Error analysis of heat pulse method for measuring soil heat capacity, diffusivity, and conductivity. Dep. of Agronomy, Kansas State Univ., Manhattan, KS 66506 CSIRO Division of Soils, PMB, PO Aitkenvale, Townsville, QLD 4814, Australia, Vol. 59, pp. 719-726. 1995.

Lam, D., Theoretical and experimental study of single-needle thermal conductivity probe. Department of Mechanical and Industrial Engineering, Ryerson University, M.A.Sc. Thesis, 2013.

Leong, W. H., Benchmark experiments on natural convection heat transfer across a cubical cavity, department of mechanical engineering, University of Waterloo, Ph.D. Thesis, pp. 145-151, 1996.

Luikov, A. V., Heat and mass transfer in capillary-porous bodies. In "Advances in Heat Transfer”, Eds): Irvine, Jr. T. F., and Hartnett, J. P., pp. 123-184, 1964.

Martynov, G.A. Heat and moisture transfer in freezing and thawing soils, in principles of Geocryology, National research council of Canada, technical translation 1065, Chapter 5, 1959.

Milly, P. C. D., Moisture and heat transport in hysteretic, inhomogeneous porous media: a matric head-based formulation and a numerical model, Water Resources Research, Vol. 18, pp. 489-498, 1982. 
Moukalled, F. and Saleh, Y., Heat and mass transfer in moist soil, Part I. Formulation and Testing, Numer. Heat Transfer B, Vol. 49, pp. 467-486, 2006.

Noborio K., McInnes K.J., Heilman J.L., Two-dimensional model for water, heat and solute transport in furrow-irrigated soil: Part I, Theory, Part II Field evaluation. Soil Sci. Soc. Am. J., Vol. 60, pp. 1001-1021, 1996.

Philip, J. R. and de Vries, D. A., Moisture Movement in Porous Materials under Temperature Gradients, Trans. Am. Geophys, Union, Vol. 38, pp. 222-232, 1957.

Ren, T., Noborio, K., and Horton, R., Measuring soil water content, electrical conductivity, and thermal properties with a thermo-time domain reflectometry probe. Soil Sci. Soc. Am. J. Vol. 63, pp. 450-457, 1999.

Ren, T., Ochsner, T. E., Horton, R., and Ju, Z., Heat-Pulse method for soil water content measurement: Influence of the specific heat of the soil solids. Soil physics. Division s-1. Vol. 67, 2003.

Ren, T., Ju, Z., Gong, Y., and Horton, R., Comparing heat-pulse and Time Domain Reflectometry soil water contents from Thermo-Time Domain Reflectometry probes. Soil Sci. Soc. Am. J. Vol. 4 pp. 1080-1086, 2005.

Robinson, D. A., Schaap, M. S., Jones, J. M. Wraith, D., and Friedman, S. P., A review of advances in dielectric and electrical conductivity measurement in soils using time domain reflectometry. Vadose Zone J. Vol. 2, pp. 444-475, 2003.

Schwartz, R. C., Casanova, J. J., Bell, J. M., and Evett, S. R. A re-evaluation of time domain reflectometry propagation time determination in soils. Vadose Zone J. 13(1), 2013.

Schönenberger, J., Momose, T., Wagner, B., Leong, W.H., Tarnawski, V.R., Canadian Field Soils I. Mineral Composition by XRD/XRF Measurements. Int. J. Thermophys. Vol. 33, pp. 342-362, 2012. 
Tarnawski, V. R., Momose, T., Leong, W. H., Bovesecchi, G., Coppa, P., Thermal conductivity of standard sands. Part I. Dry-State Conditions. Int J Thermophys. Vol. 30, pp. 949-968, 2009.

Tarnawski, V. R., Momose, T., Leong, W. H., Thermal conductivity of standard sands. Part II. Saturated conditions. Int. J. Thermophys. Vol. 32, pp. 984-1005, 2011.

Tarnawski, V. R., McCombie, M. L., Leong, W. H., Wagner, B., Momose, T., Schönenberger, J., Canadian Field Soils II. Modeling of Quartz Occurrence. Int. J. Thermophys. Vol. 33, pp. 843863, 2012.

Tarnawski, V. R., McCombie, M., Momose, T., Sakaguchi, I., Leong, W. H., Thermal conductivity of standard sands Part III. Full range of saturation. Int. J. Thermophys. Vol. 34, pp. 1130-1147, 2013.

Tarnawski, V. R., Momose, T., McCombie, M. L., Leong, W. H., Canadian field soils Part III. Thermal-conductivity Data and Modeling. Int. J Thermophys. Vol. 36, pp. 119-156, 2015.

Thomas, H. R., and King, S. D., Coupled heat and mass transfer in unsaturated soil a potential based solution. Inter. J. for numerical \& analytical methods in geo-mechanics Vol. 16 pp. 757773, 1992.

Topp, G. C., Davis, J. L. and Annan, A. P. Electromagnetic determination of soil water content: Measurements in coaxial transmission lines. Water Resour. Res. Vol. 16, pp. 574-582, 1980.

Wang, Z., Kojima, Y. Lu, S. Chen, Y., Time domain reflectometry waveform analysis with second-order bounded mean oscillation. Soil Sci. Soc. Am. J. Vol. 78, pp.1146-1152, 2014.

Welch, S. M., Kluitenberg, G. J., and Bristow, K. L., Rapid numerical estimation of soil thermal properties for a broad class of heat pulse emitter geometries, Measurement Science and Technology, Vol. 6 pp. 932-938, 1996. 